\title{
MARCEL CHADEYRAS
}

Essai d'une théorie noethérienne homogène pour les anneaux commutatifs dont la graduation est aussi générale que possible

Mémoires de la S. M. F., tome 22 (1970)

<http://www.numdam.org/item?id=MSMF_1970_22_3_0>

(C) Mémoires de la S. M. F., 1970, tous droits réservés.

L'accès aux archives de la revue « Mémoires de la S. M. F. » (http://smf. emath.fr/Publications/Memoires/Presentation.html) implique l'accord avec les conditions générales d'utilisation (http://www.numdam.org/conditions). Toute utilisation commerciale ou impression systématique est constitutive d'une infraction pénale. Toute copie ou impression de ce fichier doit contenir la présente mention de copyright.

\section{Numdam}


Bull. Soc. math. France, Mémoire 22, 1970, 143 p.

ESSAI D'UNE THÉORIE NOETHÉRIENNE HOMOGÈNE

POUR LES ANNEAUX COMMUTATIFS DONT LA GRADUATION

EST AUSSI GÉNERALE QUE POSSIBLE

par

$$
\text { Marcel CHADEYRAS }\left({ }^{*}\right)
$$

TABLE DES MATIERES

INTRODUCTION. . . . . . . . . . . . . . . . . . . . . ${ }^{2}{ }^{\text {P . . }}$

CHAPITRE I

LES ANNEIDES ET CERTAINES DE LEURS PROPRIETES ELEMENTAIRES

1 - Notions d'annétde et de modulorde. . . . . . . . . . . . . . 20

2 - Anneaux et modules associés. Sous-annéldes et sous-moduloldes. . . 23

3 - La notion de graduation. . . . . . . . . . . . . . . . 25

4 - Quotients - Morphismes . . . . . . . . . . . . . . 29

CHAPITRE II

PHENOMENE D'AGGLUTINATION. ANELS ET MONELS

1 - Le phénomène d'agglutination. . . . . . . . . . . . . 34

2 - Anels et monels. . . . . . . . . . . . . . . . . . . 37

3 - structure des agglutinés. . . . . . . . . . . . . . . 47

4 - Définition des polynômes. .................. 61

CHAPITRE III

HOMOMORPHISMES. IDEAUX ET SOUS-MONELS

1 - Propriétés des homomorphismes. . . . . . . . . . . . . . 67

2 - Opérations sur les idéaux. . . . . . . . . . . . . . . . 74

3 - Idéaux premiers, primaires, maximaux. . . . . . . . . . . . 77

4 - Anels de fractions. . . . . . . . . . . . . . . . . 83

5 - Idéaux larges. . . . . . . . . . . . . . . . . 92

(*) Thèse Sc. Math. Clermont-Ferrand, 1969. 
CHAPITRE IV

PROPRIETES NOETHERIENNES GENERALES

1 - Anels et monels noethériens. . . . . . . . . . . . . . . 97

2 - Le théorème de Hilbert. . . . . . . . . . . . . . . . 102

3 - Monotdes noethériens. . . . . . . . . . . . . . . . 109

CHAPITRE V

ANNEIDES NOETHERIENS FORTS

1 - Décomposition primaire. . . . . . . . . . . . . . . 121

2 - Intersection des puissances d'un idéal. . . . . . . . . . . . 125

3 - Annérdes de fractions. . . . . . . . . . . . . . . . 134

BIBLIOGRAPHIE. . . . . . . . . . . . . . . . . . 141

INDEX DES NOTATIONS • . . . . . . . . . . . . . . . . 142

INDEX TERMINOLOGIQUE. . . . . . . . . . . . . . . . . . 142 
Ce travail est consacré à l'étude des anneaux gradués commutatifs à graduation aussi générale que possible, dont les idéaux homogènes satisfont à la condition noethérienne : toute châ̂ne strictement croissante d'idéaux homogènes est finie (on verra que cela n'implique nullement la condition analogue pour les idéaux quelconques). Pour faire comprendre l'expression "graduation aussi générale que possible", rappelons d'abord les diverses significatıons que le terme "anneau gradué" a eues jusqu'à présent en Algèbre. Dès le début de la théorie des anneaux sont apparus les premiers spécimens de cette notion, tels ies anneaux de polynômes ou de séries formelles à plusieurs variables. Bien plus tard, quand d'autres anneaux du même type sont apparus (tels les anneaux de classes d'homologie et de cohomologie) la notion générale de grade s'est dégagée comme synthèse des notions particulières comme le degré (total, ou par rapport à l'ensemble des variables) d'un monôme, ou le poids d'un monôme lorsqu' on attribue un poids à chaque variable. Et on a appelé anneaux gradués des anneaux commutatifs o' certains éléments a non nuls, dits homogènes, sont munis d'un grade $\omega(\mathrm{a})$; tout élément non nul d'un tel anneau est somme libre d'éléments homogènes. Ces grades (pour lesquels $a b \neq 0$ entraine $\omega(\mathrm{a} b)=\omega(\mathrm{a})+\omega(\mathrm{b})$ ) étaient toujours supposès parcourir une partie stable d'un groupe additif de type $\mathbb{Z}$ ou $\mathbb{Z}^{n}$. (0 est parfois considéré comme homogène de grade ou indéterminé, ou absorbant $\infty$ ). D'autres anneaux gradués sont apparus ; par exemple les squelettes des corps valués, cas particulier des corpoides [3], qui sont parties homogènes de certains anneaux, gradués par un groupe quelconque, commutatif ou non. Pour certains anneaux, les grades parcourent un ensemble plus général muni d'une loi de composition interne.

Jusqu'ici, dans les cas concrets d'anneaux gradués rencontrés dans diverses branches mathématiques, les grades parcourent toujours une partie convenable d'un groupe commutatif. Aussi, N.Bourbaki définit la notion d'anneau gradué dans le seul cas où les grades parcourent une partie d'un monorde ou demi-groupe (ensemble muni d'une loi interne associative) commutatif, avec élément neutre. Et, de plus, il se restreint souvent au cas où le monoIde est simplifiable (c'est-à-dire a tous ses éléments réguliers), ou même est un groupe. Notons qu'il définit d'abord un groupe G commutatif gradué de type $\Delta$ (où $\Delta$ est un ensemble quelconque) comne une décomposition de $\mathrm{G}$ en somme directe de sous-groupes : $G=\underset{\lambda \in \Delta}{\oplus} G_{\lambda}$.

Les définitions de N.Bourbaki appellent d'autres remarques. 
L'ensemble des grades des éléments non nuls d'un anneau gradué est une partie d'un monoide commutatif $\Delta$, noté additivement, et ayant un élément neutre 0 . N.Bourbaki admet que certains grades $\delta \in \Delta$ puissent etre "vides", c'est-à-dire qu'aucun élément non nul ne soit de grade $\delta$. Ce point de vue sera adopté dans ce travail, car il a des avantages certains. D'une part, on pourra considérer, simultanément, toute une famille d'anneaux gradués tels que les grades des éléments non nuls de chaque anneau parcourent une partie (dépendant de l'anneau) d'un même ensemble. D'autre part, considérons un seul anneau gradué. Il peut arriver (par exemple dans les gradués de certains anneaux locaux) que, pour certains grades $\alpha$ et $\beta$, quels que soient les éléments a de grade $\alpha$ et $b$ de grade $\beta$, on ait $a b=0$. Dans ce cas, la structure de l'anneau n'indique pas quel peut être le composé des grades $\alpha$ et $\beta$, ce que cachait la donnée préalable du composé par la donnée du monorde. Si, inversement, on part de la seule structure d'anneau dont le groupe additif est gradué par l'ensemble $\Delta$, on peut ou bien supposer non défini le composé de $\alpha$ et $\beta$, et la loi interne sur $\Delta$, non partout définie, sera malcommode. Ou bien on peut prendre pour composé l'un quelconque des grades $\delta \in \Delta$; mais alors $\Delta$ sera-t-il plongeable dans un groupe, ou même seulement dans un monoIde ? Dans la structure plus générale où nous nous placerons, nous n'imposerons à la loi de composition sur $\Delta$, outre d'être partout définie, que la condition : pour tous $a, b \in A$, si $a b \neq 0, \omega(a b)$ est égal au composé de $\omega(\mathrm{a})$ et $\omega(\mathrm{b})$. Enfin, N.Bourbaki se limite par une hypothèse générale de commutativité des anneaux et de leurs monordes de grades. Cela restreint le champ d'application de la théorie; par exemple sont exclus les linéarisés (ou anneaux associés) des corpordes non commutatifs. Toutefois, ce dernier point est peu important dans la perspective de notre travail, consacré essentiellement aux anneaux commutatifs.

Rappelons donc la définition de N.Bourbaki, une des plus générales que l'on trouve actuellement. On se donne un anneau A commutatif et unitaire, un monoide $\Delta$ (que nous noterons ici multiplicativement) commutatif et possédant un élément neutre, noté 1 , et une application $\varphi$ de $\Delta$ dans l'ensemble $S(A)$ des sous-groupes du groupe additif de $A$, définie pàr $\varphi(\lambda)=A_{\lambda}$. On dit que le triple $(A, \Delta, \varphi$ ) (ou, par abus de langage, que A) est un anneau gradué de type $\Delta$, et que $\Delta$ est une graduation de $A$ si et seulement si : (1) le groupe additif de $A$ est égal à $\underset{\lambda \in \Delta}{\oplus} A_{\lambda} ;$ (2) pour tous $\lambda, \mu \in \Delta$, on a ${ }_{\lambda}{ }_{\lambda} A_{\mu}=\left\{x y ; x \in A_{\lambda}, y \in A_{\mu}\right\} \subset A_{\lambda . \mu}$. L'ensemble $H=\underset{\lambda \in \Delta}{\bigcup A_{\lambda}}$ est dit la partie homogène de I'anneau gradué $A$, et les éléments de $H$ sont dits éléments homogènes de A.

Cette nouvelle structure est donc construi te à partir de structures 
données, en leur imposant certaines conditions de coordination. Mais, on voit aisément que la donnée de la partie hornogène $\mathrm{H}$, munie de l'addition et de la multiplication induites par celles de A, suffit à déterminer, à une isomorphie près, presque tout le reste de la structure. En effet, deux éléments non nuls $a, b$ de $H$ appartiennent à un même $A_{\lambda}$ si et seulement si leur somme est dans $H$;

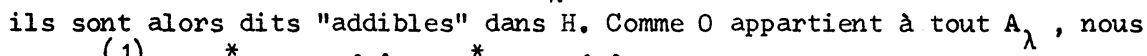
notons $(1) \quad A_{\lambda}^{*}=A_{\lambda} \cdot\{0\}$ et $H^{*}=H_{*} .\{0\}$. L'addibilité est une relation d'équivalence dans $H^{*}$ dont les classes sont les $A_{\lambda}^{*}$ non vides. Ainsi, le support et la structure additive de $H$ déterminent, indépendamment de $\Delta$ et $\varphi$, les $A_{\lambda}^{*}$ non vides, les $A_{\lambda}=A_{\lambda}^{*} U\{0\}$ correspondants, et l'ensemble $D^{*}(H)=\left\{A_{\lambda} ; \lambda \in \Pi\right\}$ de ces derniers (on désigne par $\Pi$ l'ensemble des grades $\lambda$ non vides). Puisque $(A, \Delta, \varphi)$ est un anneau gradue, chaque $\mathrm{A}_{\lambda}$ est un groupe abélien pour $I^{\prime}$ addition induite par celle de $\mathrm{H}$, et $\lambda \neq \mu$ implique $A_{\lambda} \cap A_{\mu}=\{0\}$; le groupe additif de A est la somme directe $\underset{\lambda \in \Pi}{\oplus} A_{\lambda}$. En vertu de la condition (2) de la définition, la multiplication est partout définie sur $\mathrm{H}$; elle est aussi commutative, associative, et distributive par rapport à l'addibilité et à l'addition de $\mathrm{H}$; elle définit, par distributivité, la multiplication dans $\mathbf{A}=\underset{\lambda}{\oplus \mathbf{A}_{\lambda}} \cdot$ Comme $\bar{\varphi}^{1}: A_{\lambda} \mapsto \lambda$ est une bijection de $D^{*}(H)$ sur $\Pi$, cet ensemble de grades non vides est défini, à une bijection près, par la dónnée de H. De plus, si $\lambda, \mu \in \Pi$ sont tels que $A_{\lambda} \mathbf{A}_{\mu} \neq\{0\}$, leur composé $\lambda \mu$ est déterminé par la structure de $H$, comme I'unique $\delta \in \Pi$ tel que $A_{\lambda} A_{\mu} \subset A_{\delta}$. Donc $\Pi$ est défini, par la structure de $H$, à une isomorphie près pour la composition des grades $\lambda, \mu$ tels que $A_{\lambda} A_{\mu} \neq\{0\}$.

Les seuls caractères que la structure de $\mathrm{H}$ ne détermine pas sont 1 'ensemble $\Delta . . \Pi$ des grades vides et la composition des grades $\lambda, \mu \in \Delta$ tels que $A_{\lambda} A_{\mu}=\{0\}$. Ces caractères $n$ 'ont pratiquement aucun rôle dans l'anneau gradué. Aussi il semble naturel, dans la structure d'anneau gradué, de ne considérer comme essentiel que ce qui est déterminé par la donnée de $H$ et de sa structure. Ainsi, deux structures $(A, \Delta, \varphi)$ et $\left(A ; \Delta^{\prime} \varphi^{\prime}\right)$ pourront être considérées comme un même anneau gradué si leurs parties homogènes respectives $H$ et $H^{\prime}$, munies de leurs addition et multiplication, coincident. Cela établit un isomorphisme canonique entre $A$ et $A^{\prime}$, que $I^{\prime}$ on considérera comme une identification. Quant aux applications $\varphi: \Delta \rightarrow D(H)=D^{*}(H) \cup\{\{0\}\}$ et $\varphi^{\prime}: \Delta^{\prime} \rightarrow D(H)$, on les considère comme différentes graduations possibles de cet anneau gradué (on pourra les dire graduations équivalentes de $I^{\prime}$ anneau abstrait $A=A^{\prime}$ ).

(1) On notera E..F, et non E - F, la "difference ensembliste" $\{x \in E ; x \notin F\}$, afin d'éviter toute interférence avec les notations algébriques. Pour tout ensemble $\mathrm{E}$ possédant un élément "zéron noté 0 , on posera généralement $\mathrm{E}^{*}=\mathrm{E} . .\{0\}$. 
Ce procédé est analogue à celui concemant les valuations : on considère deux valuations (au sens de M.Krasner, [3], exposé 3) d'un même corps $K$ comme équivalentes si l'une est puissance positive de l'autre. Et on considère une classe de valuations équivalentes comne une "place" (au sens classique), généralisation de la notion de point de la géométrie algébrique classique. On sait que l'équivalence de deux valuations peut se caractériser par l'égalité de I'anneau $\mathrm{V}$ de valuation (considéré comme anneau abstrait, et indépendamment de son inclusion dans le corps valué). K étant le corps des fractions de $V$, la stmucture de $V$ détermine $K$ à l'identification canonique près; et il détermine la "place", c'est-à-dire la famille, dépendant d'un paramètre réel positif, des valuations d'anneau $\mathrm{V}$. La donnée de $\mathrm{V}$ revient à définir les valuations considérées à une isomorphie près de leurs groupes, c'est-à-dire à définir tout ce qu'ont en commun ces valuations de la même place. La valeur particulière du paramètre, dans chaque valuation est un caractère secondaire que $\mathrm{V}$ ne foumit pas. On voit donc une certaine analogie de conception entre d'une part I'anneau gradué $A$ (au sens précédent), la structure homogène $H$, et les différentes applications $\varphi: \Delta \rightarrow D(H)$, et d'autre part le corps valué $K$, $I$ 'anneau de valuation $V$, et les différentes valeurs du paramètre dont dépend la famille des valuations équivalentes d'anneau $V$.

Cependant, comme on l'a vu plus haut, la donnée de $\mathrm{H}$ renseigne en partie sur $\varphi$. Si $\lambda, \mu \in \Delta$ sont non vides, et si $\varphi(\lambda) \varphi(\mu)=A_{\lambda} A_{\mu} \neq\{0\}$, le grade $\lambda \mu n$ 'est pas vide et $l$ 'on a $\varphi(\lambda) \varphi(\mu) \subset \varphi(\lambda \mu)$. Organisons donc $\Delta_{0}=D(H)$ par la loi partielle suivante : pour tous $S, T \in D(H)$ tels que ST $\neq\{0\}$, définissons le composé $S * T$ comme l'unique élément $U$ de $D(H)$ tel que $S T \subset U$. Alors, $\varphi$ induit une bijection de $\Pi$ sur $D^{*}(H)$ telle que $\varphi(\lambda \mu)=\varphi(\lambda) * \varphi(\mu)$ chaque fois que le composé à gauche est défini. Supposons, comme nous le ferons dans tout ce travail, que $\Delta$ possède un élément vide absorbant, noté $O$ (si un tel élément $n^{\prime}$ existe pas déjà, on peut l'adjoindre à $\Delta$ sans changer le fond du problème). Alors, $\varphi$ induit une bijection $\varphi_{0}$ de $\Pi \cup\{0\}$ sur $D(H)$ puisque $\varphi(0)=\{0\}$. On voit donc que la donnée de $\varphi$ équivaut à celle : (a) de I'inverse $\psi: D(H)=\Delta_{0} \rightarrow \Pi U\{0\}$ de sa bijection $\varphi_{0}$; (b) de l'extension de $\Pi \cup\{0\}$ en un ensemble plus grand $\Delta ;$ (c) de la fermeture de la transportée par $\psi$ de la loi partielle sur $D(H)$, en une loi partout définie sur $\Delta$, associative, et admettant l'élément absorbant 0 . Définissons une application canonique $\omega_{0}: H \rightarrow D(H)=\Delta_{0}$ par $\omega_{0}(0)=\{0\}$ et en prenant pour $\omega_{0}(a)$, lorsque $a \neq 0$, I'unique élément de $D(H)$

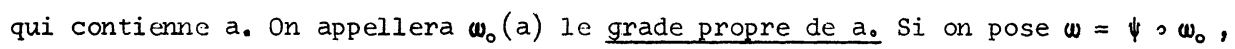
on appellera $\omega(a)$ le grade de a dans la graduation définie par $\varphi$ (ou, par abus de langage, dans la graduation $\Delta)$. H étant donnée, il est clair que la donnée 
de $\downarrow$ est équivalente à celle de $\boldsymbol{\omega}$.

Sans la condition d'associativité de la loi de $\Delta$ exigée par N Bourbaki, la graduation la plus simple de l'anneau gradué considéré aurait été la suivante : prendre $\Delta_{0}$ pour ensemble de grades (en posant $\{0\}=0$ ), l'identité sur $\Delta_{0}$ pour application $\psi$, et, lorsque $S$ et $T$ dans $\Delta_{0}$ sont tels que $S T=\{0\}$, poser $S * T=0$. Si la loi sur $\Delta_{0}$ est associative, donc satisfait à la définition de $N$. Bourbaki, on peut l'appeler graduation propre de I'anneau gradué. La multiplication de $\mathrm{H}$ étant associative, on pourrait s'attendre à priori à ce qu'il en soit toujours ainsi. Mais dans cette analyse de la notion d'anneau gradué au sens de $N$. Bourbaki que nous a suggérée M. Krasner, nous avons constaté que la loi sur $\Delta_{0} n^{\prime}$ est pas forcément associative, même en choisissant autrement les composés $\mathrm{S} * \mathrm{~T}$ lorsque $\mathrm{S} \mathrm{T}=\{0\}$. Et cela arrive mêne dans des cas simples.

Revenons à l'analogie avec la théorie des valuations. Dans cette théorie, l'anneau $\mathrm{V}$ de valuation est déterminé par la structure plus compliquée de son corps valué de fractions; et $\mathrm{V}$ permet de reconstruire cette structure, à l'équivalence de valuation près. On renverse ensuite le procédé, en caractérisant axiomatiquement tout anneau abstrait qui est anneau de quelque valuation de son corps de fractions; on obtient, à partir de cette classe d'anneaux, toutes les places possibles par la construction rappelée plus haut. M. Krasner nous a suggéré d'appliquer une méthode analogue à la théorie des anneaux gradués (caractériser axiomatiquement toute partie homogène $\mathrm{H}$ qui est celle de quelque anneau gradué; puis obtenir à partir de là tous les anneaux gradués possibles), en débarrassant la définition de N. Bourbaki des restrictions qui n'empêchent pas la construction à effectuer, et des conditions concernant la partie non essentielle de la structure. On abandonne donc I'hypothèse de commutativité de l'anneau $A$ et de l'ensemble $\Delta$ des grades, ainsi. que I'existence d'un élément neutre dans $\Delta$. (Cependant, ces hypothèses seront reprises dans la majeure partie de ce travail, consacré aux anneaux commutatifs). Mais surtout, on n'imposera des conditions qu'aux seuls grades non vides, et on n'imposera I'associativité qu'aux triples $\alpha, \beta, \gamma$ tels que $A_{\alpha} A_{\beta} A_{\gamma} \neq\{0\}$. Bien entendu, on maintient les conditions : (1) $A=\oplus_{\lambda \in \Pi} A_{\lambda}$ (en éliminant les grades vides); (2) $A_{\alpha} A_{\beta} \subset A_{\alpha \beta}$. On remarque alors que, lorsque $\mathrm{A}_{\alpha} \mathrm{A}_{\beta} \mathrm{A}_{\gamma} \neq\{0\}, I^{\prime}$ associativité du produit de $\alpha, \beta, \gamma$ vient de (2) et de I'associativité dans I'anneau.

Ainsi, on appellera finalement anneau gradué au sens le plus général la structure $(A, \Pi, f)$ suivante : A est un anneau arbitraire, $\Pi$ est un ensemble muni d'une loi de composition en général partielle, et $f$ est une application de $\Pi$ dans $I$ 'ensemble $S^{*}(A)$ des sous-groupes $\neq\{0\}$ du groupe 
additif de $A$. Ces données vérifient les axiomes : $\left(\psi \alpha \in \Pi\right.$, on notera $\left.f(\alpha)=A_{\alpha}\right)$ : $1^{\circ} \forall \alpha, \beta \in \Pi, \alpha \beta$ est défini si et seulement si $A_{\alpha} A_{\beta} \neq\{0\} ; 2^{\circ}$ si $\alpha \beta$ est défini, on a $A_{\alpha} A_{\beta} \subset A_{\alpha \beta} ; 3^{\circ}$ le groupe additif de $A^{\text {est }} \underset{\lambda \in \Pi}{\oplus} f(\lambda)=\underset{\lambda \in \Pi}{\oplus}{ }_{\lambda}{ }^{*}$ On appelle partie homogène de cet anneau l'ensemble $H=\bigcup_{\lambda \in \Pi}{ }_{\lambda}$ organisé par I'addition partielle et la multiplication induites par celles de A. On considère que les structures $(A, \Pi, f)$ et $\left(A, \Pi^{\prime}, f^{\prime}\right)$ sont un même anneau gradué s'il existe un isomorphisme $\eta: \Pi^{\prime} \rightarrow \Pi$ tel que $f^{\prime}=f$ 。 $\eta_{0}$. Une manière canonique de définir $\Pi$ et $f$ est de prendre pour $\Pi I^{\prime}$ ensemble $D^{*}(H)=\left\{A_{\lambda} ; \lambda \in \Pi\right\}$ (il est indépendant du choix de $\Pi$ ), muni de la loi $S_{* T}$ (avec $S, T \in D^{*}(H)$ ) définie si

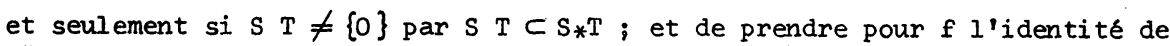
$D^{*}(H)$. On définira les graduations possibles de 1 'anneau gradué considéré comme les couples $(\Delta, \varphi)$ où $\Delta$ est un ensemble muni d'une loi de composition partout définie et ayant un élément absorbant 0 , et où $\varphi$ est une application $\Delta \rightarrow \Delta=D(H)$, injective sur I'image réciproque $\bar{\varphi}^{-1}\left(D^{*}(H)\right)$, et telle que $\varphi(0)=\{0\}$ et que $\varphi(\alpha) \varphi(\beta) \subset \varphi(\alpha \beta)$ pour tous $\alpha, \beta \in \Delta$. (On dira souvent : la graduation $\Delta, \varphi$ étant sous-entendue).

Il semblerait alors souhaitable, pour la commodité, de restreindre le choix de $\Delta$ aux cas où sa loi est associative (nous dirons : aux graduations associatives). Ce fut un étonnement (partagé, je crois, par MM. Krasner et Samuel) de constater qu'un anneau gradué (au sens précédent $(A, \Gamma, f)$ ) peut très bien n'avoir aucune graduation associative. On le voit dans certains cas très simples, lorsque la loi partielle de $\Pi$ n'est déjà pas associative. Ce phénomène ne peut se produire que pour des triples de grades $\alpha, \beta, \gamma \in \Pi$ tels que $A_{\alpha} A_{\beta} A_{\gamma}=\{0\}$; si les produits $A_{\alpha} A_{\beta}$ et $A_{\beta} A_{\gamma}$ sont différents de $\{0\}$, et s'il en est de même de $A_{\alpha \beta}{ }^{A} \gamma$ et de $A_{\alpha} A_{\beta} \gamma$, les produits $(\alpha \beta) \gamma$ et $\alpha(\beta \gamma)$ sont définis dans $\Pi$; mais rien ne les oblige à être égaux, comme on le montrera. On verra même un exemple d'anneau gradué où $\Pi$ est un ensemble fini muni d'une loi partout définie et commutative, mais, à part cela, quelconque. Ce demier exemple, il est vrai, est tératologique ; il s'étend au cas où II est dénombrable. On est donc obligé d'abandonner toute condition préalable d'associativité sur les grades. Donc, un anneau gradué au sens considéré possèdera toujours une graduation propre $\Delta_{0}$, qui sera ou non associative. On verra que la plus grande partie de ce travail est indépendante de l'associativité de la graduation. On verra par contre certains résultats importants ou I'hypothèse d'associativité semble indispensable. Aussi, on considèrera de préférence les graduations associatives, s'il en existe. De même, nous éviterons toute complication artificielle, lorsque la classe d'anneaux gradués le permet : Pour les anneaux commutatifs, nous nous limiterons à leurs graduations 
commutatives. Pour les anneaux unitaires où 1 est homogène, nous nous limiterons presque toujours aux graduations $\Delta$ où le grade de 1 est neutre.

Passons à la caractérisation axiomatique de la partie homogène $H$ de ces anneaux gradués. H satisfait évidemment aux axiomes suivants (a addible à $\mathrm{b}$ sera noté $\mathrm{a} \# \mathrm{~b}$ ) :

I. $\mathrm{H}$ est un monorde par rapport à sa multiplication, et possède un élément (bilatéralement) absorbant 0 .

II. $O$ est addible avec tout élément de $\mathrm{H}$; I'addibilité est réflexive, symétrique ( $\mathrm{a} \# \mathrm{~b}$ implique $\mathrm{b} \# \mathrm{a}$ ) et presque transitive ( $\mathrm{si} \mathrm{b} \neq 0$, les relations $\mathrm{a} \# \mathrm{~b}$ et $\mathrm{b} \# \mathrm{c}$ impliquent $\mathrm{a} \# \mathrm{c}$ ). Pour tout élément a non nul de $H, l^{\prime}$ ensemble $A(a)=\{x \in H ; x \not A\}$ est un groupe abélien pour l'addition induite par celle de $\mathrm{H}$.

III. La multiplication est bilatéralement distributive par rapport à l'addibilité et à l'addition : pour tout $c \in \mathrm{H}, \mathrm{a} \# \mathrm{~b}$ implique ca \# $\mathrm{cb}$, $a c \# b c, c(a+b)=c a+c b,(a+b) c=a c+b c$.

Inversement, lorsqu'un ensemble $\mathrm{H}$, muni d'une multiplication et d'une addition partielle, vérifie ces axiomes, on montre aisément qu'il est la partie homogène d'un anneau gradué : L'élément neutre $O_{a}$ de tout gruupe $A(a)$ est 0 ; en effet $0 \in A(a)$, et comme $o_{a}$ se caractérise par $0_{a}=0_{a}+0_{a}$, on en déduit $0=00_{a}=0\left(0_{a}+0_{a}\right)=00_{a}+00_{a}=0+0$, d'où $0=0_{a}$.

Sur $\mathrm{H}^{*}=\mathrm{H} . .\{0\}, I^{\prime}$ addibilité est évidemment une relation d'équivalence ; ainsi, les $A^{*}(a)=A(a) \ldots\{0\}$ distincts forment une partition de $H^{*}$, et les groupes additifs $\mathrm{A}(\mathrm{a})$ distincts ont deux à deux en commun le seul élément 0 . Si donc $D^{*}(H)$ est $I^{\prime} e n s e m b l e$ de ces groupes, on prendra leur somme directe $\mathrm{G} \stackrel{\oplus}{\oplus} \mathrm{D}^{*}(\mathrm{H})$ G pour groupe additif de I'anneau A à construire. On y définit la multiplication à partir de celle de $\mathrm{H}$, grâce à sa nécessaire distributivité bilatérale par rapport à l'addition dans A. Alors, l'associativité et la distributivité pour l'addition de la multiplication dans A se déduisent immédiatement des propriétés d'associativité et de distributivité de celle de $\mathrm{H}$. De plus, si la multiplication de $\mathrm{H}$ est commutative, celle de A l'est aussi ;

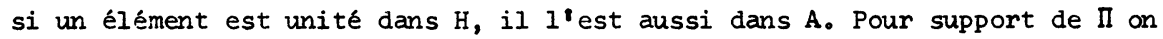
prendra $D^{*}(H)$, et pour $f I^{\prime a p p l i c a t i o n ~ i d e n t i q u e . ~ S e u l e s ~ l e s ~ c o n d i t i o n s ~} 1^{\circ}$ et $2^{\circ}$ restent a remplir. Pour tous $S, T \in D^{*}(H)$, tous $a, a^{\prime} \in S$ et tous $b, b^{\prime} \in T$, les conditions $a \# a^{\prime}, b$ \# $b^{\prime}$ impliquent $a b$ \# $a^{\prime} b^{\prime}$. En effet, elles entrainent par distributivité $a b \# a^{\prime} b$ et $a^{\prime} b \# a^{\prime} b^{\prime}, a b^{\prime} \not a^{\prime} b^{\prime}$ et $a b \# a b^{\prime} ;$ si $a b^{\prime} \neq 0$, ou si $a^{\prime} b \neq 0$, la presque transitivité implique $a b \# a^{\prime} b^{\prime} ;$ sinon, la conclusion est encore vraie, selon une remarque de M. Krasner, car on a alors $a b=a\left(b+b^{\prime}\right) \not a^{\prime}\left(b+b^{\prime}\right)=a^{\prime} b^{\prime}$. En particulier, dans tous les cas où $\mathrm{S} T \neq\{0\}$, il existe un unique élément $U \in D^{*}(H)$ tel que $S T \subset U$; et on 
prendra alors par définition $U$ comme composé de $S$ et $T$. Les conditions $1^{\circ}$ et $2^{\circ}$ sont alors vérifiées.

La structure $H$ satisfaisant aux axiomes I, II, III s'appelle annérde. Elle a été introduite par M. Krasner [4], qui l'a précisée dans ses cours et séminaires. Ainsi, le noyau ultime et intrinsèque de la notion d'anneau gradué est la notion d'annélde. Dès lors, deux manières d'étudier les anneaux gradués sont possibles : à partir de l'anneau et d'une de ses graduations, comme l'ont fait jusqu'ici la plupart des auteurs. Ou bien à partir de l'annéIde H, méthode adoptée par M. Krasner dans la théorie des corpolaes [4], et qui sera généralement adoptée dans ce travail. (Certains résultats seront reformulés en termes d'anneaux gradués). Dans la première méthode, l'addition est partout définie. Mais cet avantage est plus apparent que réel dans bien des cas : d'abord la non-addibilité d'éléments de $\mathrm{H}$ se retrouve dans la non-homogénélté de leur somme. Mais surtout, les notions homogènes sont obtenues en imposant, aux notions correspondantes de l'anneau abstrait, certaines conditions supplémentaires d'homogénélté. Aussi intervient une foule d'éléments non-homogènes, qui probablement sont souvent superflus ou parasites. Ils risquent d'alourdir certaines propriétés ou démonstrations de la théorie, en estompant ce qui est essentiel. Dans la deuxième méthode, I'annélde $\mathrm{H}$ détermine toute la structure, à quelques caractères secondai près. Donc, toutes les notions doivent pouvoir se formuler en termes d'éléments homogènes, ainsi que les démonstrations. Si parfois, quelques éléments non-homogènes peuven y figurer à titre auxilialre, cette méthode doit permettre de "doser" leur emploi, en se limitant chaque fois à ceux qui sont nécessaires.

Il existe un autre avantage plus concret de la deuxième méthode : A étant un anneau commutatif gradué, considérons une partie multiplicativement stable $M($ avec $0 \notin M$ ). Si $M n$ 'est pas un ensemble d'éléments homogènes, en général I'anneau $A_{M}$ des fractions ne possèdera pas de graduatior naturellement déduite de celle de A. En effet, I'inverse d'un élément non-homogène n'est pas, en général, une somme de quotients d'éléments homogènes. Pour cette raison, le vrai "corps de fractions homogène" de A (si A est sans diviseur de 0) n'est pas $\mathbf{A}_{\mathbf{A}}{ }^{*}$, mais $\mathbf{A}_{\mathbf{H}^{*}}$. Et le vrai "anneau localisé homogène de A par rapport à un idéal $p$ homogène premier" $n$ 'est pas $A_{A} \ldots p$, mais $\epsilon_{H, .}(p \cap H)$ • Ainsi, le vrai domaine de la théorie des fractions dans 1 'homogène est 1 'annélde $H$, et non I'anneau A. Ceci sst particulièrement visible dans le cas des corpolder. $c^{\prime}$ est-à-dire des annéldes $Q$ où $Q^{*}=Q_{.} \cdot\{0\}$ est un groupe multiplicatif. Dans la théorie des annéldes, ce sont eux les véritables "corps dans I'homogène" (on a $Q=Q_{Q^{*}}$ ) ; et leurs propriétés sont analogues à celles des corps (voir [4]). 
Par contre, si $A=\bar{Q}$ est $I^{\prime}$ anneau gradué associé à $Q$, on a $A_{Q^{*}}=A$, tandis que $A_{A^{*}}$ (attention, A peut n'être pas intègre : I, $\S 2$, ex. 2) n'a pas une structure homogène favorable. Toutes ces raisons nous ont fait préférer la deuxième méthode comme néthode de base. Bien entendu, ceci ne se rapporte qu'à la théorie générale des anneaux gradués, et pas forcément à certaines applications de ces anneaux. Lorsque des éléments non-homogènes interviennent effectivement, la première méthode s'impose; c'est le cas, par exemple, des anneaux de polynômes appliqués à la géométrie algébrique affine.

D'ailleurs, M. Krasner a introduit la notion d'annéIde comme une généralisation de la notion de corpolde [4] (analogue au passage de la notion de corps à celle d'anneau), et non comme partie homogène d'un anneau gradué. Mentionnons toutefois qu'une classe, assez particulière, d'annéldes a été envisagée indépendamment par Sagastume Berra [7], à partir des anneaux gradués. Quant à la notion de corpolde, introduite également par M. Krasner en 1944, elle a son origine dans la théorie des corps valués (voir [3], squelette d'un corps valué). Jans son Séminaire [4], M. Krasner a développé la théorie des corpoides commutatifs et "sans torsion" (c'est-à-dire dont le groupe des grades est sans torsion). Il a montré qu'ils admettent une théorie des extensions, d'algébricité, de séparabilité, de transcendance aussi précise que celle de Steinitz pour les corps. Mais cette théorie est plus riche, car l'extension d'un tel corpoide peut se manifester en partie par l'extension de son corps (ce corps est I'ensemble des éléments addibles à 1), et en partie par celle de son groupe (des grades). M. Krasner a montré (2) que les extensions finies de tels corpoldes admettent une théorie de Galois de même type que celle des corps (sauf que la deuxième partie du théorème de Galois se démontre par d'autres méthodes, car le théórème de l'élément primitif n'est plus valable). Dans I'exposé cité [4], M. Krasner développe d'ailleurs la théorie des annéIdes des polynômes (d'un ensemble arbitraire de variables) sur un tel corpolde q. En effet, si on fixe les grades des variables (qu'on suppose, dans ce cas, pris dans un surgroupe abélien sans torsion du groupe de q), les polynônes seront, par définition, les sommes formelles de monômes d'un même grade, et on peut montrer que ces polynames forment un annérde. Ces annérdes (qui sont factoriels), jouent le même rôle dans la thénrie des extensions corpordales que les anneaux de polynômes dans celle des extensions de corps. Toute la théorie élémentaire des anneaux commutatifs (idéaux, anneaux quotients, corps de fractions) reste rraie, avec des changements minimes (et en remplaçant, bien entendu, les corps

(2) Cette théorie n'est pas encore publiée. Elle a été plusieurs fois exposée dans ses cours de Clermont-Ferrand, 1961-62, de Paris 1966-57, ainsi que dans son séminaire sur la théorie des corps valués. 
par les corpoides) tans ces annérdes. Elle se transporte d'ailleurs aux annérdes quelconques.

On peut envisager les anneaux gradués $\bar{q}$ et $\bar{q}[\mathrm{X}]$ qui correspondent à ces corpoides $q$, ou à ces annérdes de polynômes $q[X]$. On a vu que cela est plutôt une complication, à cause des éléments non homogènes; de plus, ces derniers masquent $l$ 'analogie des corpordes avec les corps. D'autre part, soit $k$ un corps valué, et soit $S$ son squelette, qui est un corporde; là, rien ne correspond, dans la structure du corps valué $k$, aux éléments non homogènes de $\bar{s}$,

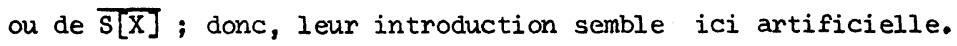

Dans la théorie des anneaux gradués existe l'analogue homogène de la notion de module. On peut la définir en partant soit du point de vue des anneaux gradués, soit de celui des annérdes. Nous nous bornerons à donner les définitions sous les deux formes; le lecteur saisira aisément leur signification.

On appelle module gradué sur un anneau gradué ( $A, \Pi, f)$ la structure $(E, \Sigma, g)$ suivante : $E$ est un A-module, $\Sigma$ est un ensemble muni d'une loi de composition externe (partielle en général) dont le domaine d'opérateurs est $\Pi$, et $g$ est une application de $\Sigma$ dans $I^{\prime}$ ensemble $S^{*}(E)$ des sous-groupes $\neq\{0\}$ du groupe additif de $E$. ( $\forall \xi \in \Sigma$, on notera $\left.g(\xi)=E_{\xi}\right)$. Ces données vérifient les axiomes : $1^{\circ} \forall \alpha \in \Pi, \forall \xi \in \Sigma, \alpha \xi$ est défini si et seulement si $f(\alpha) g(\xi)=A_{\alpha} E_{\xi} \neq\{0\} ; 2^{\circ}$ si $\alpha \xi$ est défini, on a $A_{\alpha} E_{\xi} \subset E_{\alpha \xi} ; 3^{\circ}$ le groupe additif de $E$ est $\underset{\xi \in \Sigma}{\oplus} g(\xi)=\underset{\xi \in \Sigma}{\oplus} E^{E}$. (De mêmé, dans $\Sigma$, l'associativité des scalaires pourra $n$ 'être pas vérifiée). L'ensemble $M(E)=\bigcup_{E \in \Sigma} E_{\xi}$ est dit partie homogène de E. Posons $C^{*}(M)=\left\{g(\xi)=E_{\xi} ; \xi \in \Sigma\right\}$ et $C(M) \stackrel{\xi \in \Sigma}{=} C^{*}(M) \cup\{\{0\}\}$ Si $(\Delta, \varphi)$ est une graduation de $A$, on appelle $\Delta$-graduation de $E$ tout couple $(\Gamma, \psi)$ vérifiant les conditions suivantes : $\Gamma$ est un ensemble muni d'une loi de composition exteme partout définie, dont $\Delta$ est le domaine d'opérateurs; test une application $\Gamma \rightarrow \Gamma_{0}=C(M)$, injective sur l'image réciproque $\bar{\psi}^{1}\left(C^{*}(M)\right)$, et telle que $\psi(0)=\{0\}$ et que $\varphi(\alpha) \psi(\xi) \subset \psi(\alpha \xi)$ pour tous $\alpha \in \Delta$, $\xi \in \Gamma$. Même lorsque $\Delta$ est associative, un module gradué peut $n$ 'avoir pas de $\Delta$-graduation $(\Gamma, \psi)$ dont la loi externe vérifie l'associativité des "scalaires". on appelle modulorde à gauche sur un annérde $H$ un ensemble $M$

vérifiant les axiomes suivants : I. M est muni d'une multiplication externe, dont $\mathrm{H}$ est le domaine d'opérateurs à gauche, et qui vérifie l'associativité des scalaires. $M$ possède un élément absorbant $O^{\prime}$ tel que a $O^{\prime}=O^{\prime}$ pour tout $a \in H$. ( 0 ' sera désormais noté 0 ). II. M est muni d'une addition partielle vérifiant les mêmes axiomes (II) que $H$ ( $x$ addible à $y$ est noté $x \not y)$. III. La multiplication est bilatéralement distributive par rapport aux 
addibilités et aux additions : pour tout a $\in \mathrm{H}, \mathrm{x} \# \mathrm{y}$ (avec $\mathrm{x}, \mathrm{y} \in \mathrm{M}$ ) implique ax \# ay et $a(x+y)=a x+a y$. Pour tout $x \in M, a \# b($ avec $a, b \in A$ ) implique $a x \# b x$ et $(a+b) x=a x+b x$. De plus, si $H$ possède un élément unité 1 , et si $1 x=x$ pour tout $x \in M$, le modulorde est dit unitaire. Le modulorde est dit régulier si, pour tout $x \in M^{*}$ et pour tous $a, b \in H$, la relation ax \#bx implique a \#b.

En particulier, si $\mathrm{H}$ est un corporde Q, un Q-modulorde unitaire V est dit un espace vectoriel sur Q. Il est dit espace vectoriel régulier ou strict, si de plus il est régulier. Les espaces vectoriels stricts sur les corpordes ont été étudiés en détail par 1. Krasner. Ils ont pratiquement toutes les bonnes propriétés des espaces vectoriels sur les corps (la dimension; par exemple), et en plus quelques autres. Par contre, les espaces vectoriels non stricts peuvent avoir des structures très compliquées.

Le présent travail se distingue de ses prédécesseurs en ce qu'il essaie d'aborder l'étude effective des anneaux commutatifs, gradués au sens général. Ceux-ci n'ont donc pas forcément pour graduation un monorde avec simplification (nous dirons, par abus de langage, une graduation associative simplifiable), ou même un monorde. Notre modèle a été "Commutative Algebra" de 0. Zariski et P. Samuel. Aussi, ce travail a été fait surtout dans la perspective noethérienne. Cependant, nous avons sciemment écarté l'étude similaire de certaines parties concernant les seuls anneaux dont les analogues homogènes possèdent toujours une graduation associative simplifiable. (Par exemple, les anneaux de Dedekind, les corps). En effet, dans l'optique générale de ce travail, leur étude parât "relativement simple", donc "en bordure" de notre théorie. Cette étude a d'ailleurs été entamée où faite sur certains points par d'autres auteurs. Par exemple, M. Krasner a étudié les corpordes et leurs extensions. C'est pourquoi nous avons laissé de côté ce qui concerme les analogues homogènes des corps, des anneaux de Dedekind, des valuations et des anneaux de polynômes et de séries formelles. Cependant, le manque de temps nous a empecchés pour l'instant d'étudier l'analogue homogène de l'algèbre locale (chap. VIII du volume II), et aussi d'essayer de voir s'il $n^{\prime} y$ a pas quelque simplification en remplaçant la considération des gradués des anneaux locaux par celle de leurs parties homogenes.

Nous esperons avoir construit une theorie homogene, sans doute non

exhaustive, mais qui, dans les limites indiquées, présente une généralité suffisante.

Dans les anneaux et modules gradués aussi généraux que nous étudions, intervient un phénomene, absent dans les cas classiques étudiés auparavant, dit d'agglutination. De lui viennent l'essentiel de l'intéret et de la difficulté. 
Par exemple, pour des éléments homogènes $a, b, c$ d'un anneau gradué, il peut arriver que $b$ et $c$ soient de grades différents, mais que $a b+a c$ soit homogène ; on dit que a agglutine $b$ et $c$. Si de plus ab et ac sont non nuls (donc de même grade), on dit que le grade de a agglutine ceux de b et de $c$. On verra d'autres cas d'agglutination, et on précisera cette notion. Ce phénomène oblige pratiquement, pour une étude convenable des anneaux gradués, à envisager une stmucture très voisine de la leur, avec une partie homogène plus grande, et une graduation moins fine que celle de départ. Dans l'exemple précédent, pour a homogène non nul fixé, un élément $x$ de $I^{\prime}$ anneau sera homogène dans la "quasi"-graduation que a définit, si ax l'est dans la graduation initiale ; alors, le "quasi"-grade de $x$ sera par définition le grade de ax (quasi-grade 0 si ax =0). Dars l'anneuu, les éléments de "quasi"-grade nul forment un idéal ; pour un grade iixé $\lambda$, l'ensemble des éléments de "quasi"-grade 0 ou $\lambda$ est un groupe additii abélien. L'anneau est la somme de ces groupes distincts, directe à I'idéal ci-dessus près (somme "quasi-directe").

On a ainsi obtenu la structure d'anneau quasi-gradué, à partir de celle d'anneau gradué, en dilatant, en quelque sorte, son zéro en un idéal. Les parties homogènes de tels anneaux, qui admettent aussi une caractérisation axiomatique, seront appelées anels. On considérera les modules quasi-gradués (sens analogue) sur de tels anneaux, et leurs parties homogènes seront appelées monels. Ces considérations conduisent aussi à une exploration, sur un anneau gradué donné, de ses graduations et quasi-graduations moins fines.

On définit, pour les anneaux gradués, les analogues des anneaux de polynômes. Là, les indéterminées elles-mêmes sont munies de grades, suivant le procédé utilisé par M. Krasner [4] pour les polynômes sur un corpolide. Ces grades seront pris dans un "prolongement" $\Delta^{\prime}$ de la graduation $\Delta$ ( $\Delta^{\prime}$ est à $\Delta$ ce que $\Delta$ est à $\Delta_{0}$ ). Alors, 1 a définition du grade d'un monôme a $x_{1}^{1} \ldots \ldots x_{p}$ (avec a homogène) est basée sur le produit des grades des facteurs, mais sans nécessiter I'associativité de la graduation $\Delta^{\prime}$. Et un polynôme est dit homogène si ses monômes de grade non nul ont même grade. Cet ensemble de polynômes homogènes, organisé de manière évidente, $n^{\prime}$ est pas, en général, un annérde (cela peut venir du grade $\xi_{i}$ d'une indéterminée $x_{i}$, qui diviserait 0 dans $\Delta^{\prime}$, ou bien entrerait dans un triple non associatif de $\Delta^{\prime}$ ). Mais cet ensemble est toujours un anel. Donc un anneau de polynômes sur un anneau gradué peut n'être pas naturellement un anneau gradué, sauf si on limite sérieusement le choix des grades $\xi_{i}$ des $X_{i}$. Pour éviter cette limitation, nous avons employé les anneaux quasi-gradués. Ces derniers sont donc utiles en-dehors des problèmes qui ont conduit à les envisager.

Dans les anneaux et les modules quasi-gradués, nous avons pu, en 
nous bornant aux parties homogènes et aux idéaux et sous-modules homogènes, construire une théorie analogue à celle des anneaux et modules (cette dernière est alors un cas particulier). Les résultats sont pour la plupart identiques ; quelques-uns nécessitent des hypothèses supplémentaires peu restrictives. Mais certaines notions sont modifiées, ou se dédoublent. Par exemple, soit $p$ un idéal homogène; dans I'homogène il est dit premier si ses éléments homogènes vérifient la condition habituelle; et il est dit largement premier si (pour tous b homogène et $\alpha$ quelconque) les conditions $\alpha$ b homogène $\in p$ et $\alpha \notin p$ impliquent $b \in p$. Soit un idéal homogène maximal $\mathfrak{m}$ (pas forcément maximal parmi les idéaux quelconques) d'un anneau quasi-gradué $R$, de graduation associative $\Delta$, et tel que $\mathrm{R}^{2} \phi \mathrm{m}$; on montre que $\mathrm{m}$ est largement premier et que la partie homogène de $\mathrm{R} / \mathrm{m}$ est un corporde.

De même, les généralités noethériennes classiques s'étendent avec peu de changements aux anneaux (et modules) quasi-gradués noethériens dans I'homogène (c'est-à-dire aux anels et monels noethériens). Cependant, si un annérde est noethérien, son anneau gradué peut ne pas l'être, comme certains de ses modulordes de type fini.

Considérons un anneau quasi-gradué et noethérien dans l'homogène (donc un annéride noethérien) s'il admet une graduation associative $\Delta$, le sous-monoide $\Gamma$ de $\Delta$ engendré par les grades non nuls est un monoide noethérien, c'est-à-dire que tout $\Gamma$-idéal de $\Gamma$ possède un système fini de $\Gamma$-générateurs. Une caractérisation explicite des monordes noethériens a été obtenue dans ce travail.

Pour la transmission de la propriété noethérienne dans l'homogène, d'un anneau quasi-gradué à celui de ses polynômes à une variable de grade $\xi$, le théorème de Hilbert ne reste valable que sous certaines conditions sur $\xi$ (même si la partie homogène donnée est un corpoíde). En particulier, ce théorème reste vrai dans 1 'homogène si de plus, $\Delta$ étant associative et $\xi$ quási-régulier (c'est-à-dire qu'il existe $i \geqslant 0$ tel que, $\forall n \geqslant i, \alpha \xi^{n}=\beta \xi^{n}$ implique $\alpha \xi^{i}=\beta \xi^{i}$ ), S divise le grade $\omega(b)$ d'un élément homogène $b$, dont l'annulateur dans l'anneau est un idéal noethérien (au sens habituel).

Dans la théorie des anneaux noethériens, un role fondamental est joué par deux théorèmes : celui de Lasker-Noether qui montre que tout idéal irréductible est primaire, et celui de Krull, qui donne des conditicis suffisantes pour que l'intersection des puissances d'un idéal soit (0). Dans la théorie homogène des anneaux gradués, le premier théorème subsiste si, outre la condition noethérienne dans I'homogène, on suppose que l'anneau gradué est fort. Fort signifie ceci : pour tout élément homogène non nul a, il existe un entier $i=i(a) \geq 0$ tel que les relations $0 \neq b a^{n} \# c a^{n} \neq 0$ (avec $b$ et $c$ homogènes, 
$\mathrm{n} \geq 0$ ) impliquent $\mathrm{b} \mathrm{a}^{i} \# \mathrm{c} \mathrm{a}^{i}$. Cette condition est vérifiée en particulier si tout grade non nul est quasi-régulier; et on montre qu'elle est étrangère à la propriété noethérienne. Par suite, la classe des anneaux gradués forts et noethériens dans I'homogène, apparât comne le domaine naturel de la théorie noethérienne hornogène. Ce résultat est important dans ce travail, d'autant plus que la classe envisagée semble suffisamment vaste.

En ce qui concerne le théorème de Krull, ses conditions se généralisent avec de légères modifications. Elles ne sont pas plus restrictives que dans la théorie habituelle des anneaux commutatifs, sauf si les anneaux gradués sont tératologiques d'un certain point de vue : plus précisément, cela peut arriver quand l'anneau gradué a trop d'éléments homogènes diviseurs de zéro.

Lors de l'étude du théorème de Xrull et de ses conséquences, dans les cas défavorables, interviennent certains idéaux homogènes étroitement liés à la graduation propre $\Delta_{0}$. Un idéal homogène est dit large en un grade non nul $\alpha$ s'il contient tous les éléments homogènes de grade $\alpha_{\text {. Il }}$ est dit large, s'il est large en tout grade dont il contient un élément non nul, et large aussi en tout multiple d'un tel grade. Lorsque l'anneau est muni de sagraduation propre $\Delta$, il existe une bijection entre l'ensemble des idéaux larges et celui des idéaux de $\Delta_{0}$. La somme de deux idéaux, dont un est large, est égale à leur réunion. Citons un exemple. Il existe un plus grand idéal homogène large L contenu à la fois dans l'ensemble de 0 et des divtseurs de 0 , et dans un idéal homogène propre a. Certaines conditions sont suffisantes dans les anneaux abstraits pour que $\stackrel{\infty}{n}_{a^{n}}^{n}=(0)$; les conditions homogènes analogues impliquent seulement $\infty^{n=1}$ $a^{n} \subset 1$. $n=1$

Ce travail contient encore une étude de localisation des anneaux gradués à l'aide de systèmes multiplicatifs homogènes (en particulier, de la partie homogène du complément d'un idéal homogène, premier dans l'homogène).

Dans le chapitre I se trouvent la définition des annérdes et modulordes et quelques généralités à leur sujet, en particulier la notion de graduation.

Le chapitre II introduit les notions d'anel et de monel, de sousmonel et d'idéal, étudie la structure des ensembles d'agglutinés, et définit les anels de polynômes.

Le chapitre III contient essentiellement les généralités sur les sous-monels et surtout sur les idéaux (des anels), en liaison avec les homomorphismes plus ou moins "homogènes". On y trouve en particulier des théorèmes d'isomorphisme, les diverses notions d'idéaux premiers ou primaires, l'étude des anels de fractions, et celle des idéaux larges. 
Le chapitre IV commence l'ćtude des anels et monels noethériens, et donne des analogues homogènes au théorène de Hilbert pour les anels de polynômes. Ce chapitre, se termine par l'analyse de la structure d'un monorde noethérien.

A partir du chapitre $V$, consacré aux seuls annéldes noethériens, on ajoute presque partout l'hypothèse que ces annéldes sont forts. Là, tout idéal irréductible est primaire, et la décomposition primaire d'un idéal existe. Cette hypothèse permet aussi une étude raisonnable du théorème de Krull : $\mathfrak{m}$ étant un idéal propre, quelles conditions suffisent pour que $\prod_{n=1}^{\infty} m^{n}=(0)$, et sinon, que peut-on dire de cette intersection? Le chapitre s'achève par quelques considérations sur des annétdes de fractions.

Je tiens à exprimer ma chaleureuse reconnaissance à Monsieur KRASNER. Il a été, dans toute la période de ce travail un tuteur sar, un exemple, et un guide toujours abordable. Mais il a été beaucoup plus. Par une action à la fois patiente et subtile, il a su me conduire dans le chemin le plus favorable à l'acquisition d'une formation et d'un esprit de recherche grace auxquels $j$ 'ai pu d'abord achever cette thèse. Qu'il sache que $j$ ! ai pleinement conscience de ma dette scientifique envers lui.

C'est grâce à Monsieur SAMUEL que j'ai pris goat à l'Algèbre commutative, lors de ses cours et séminaires à CLERMONT. L'attention qu'il a bien voulu porter à mon travail a été un puissant stimulant dans mon effort. De tout cela, je lui garde une profonde gratitude.

Monsieur LAFON a guidé avec bienveillance mes premiers pas vers la recherche. Sans ces débuts, je $n^{\prime}$ aurais peut-être pas osé entreprendre un travail plus vaste. Je considère que je lui dois beaucoup.

Je remercie sincèrement Monsieur BLANC qui a bien voulu accepter de présider mon jury, et Monsieur GUILLAUnE qui a bien voulu accepter d'en faire partie. Leurs encouragements amicaux m'ont été d'un grand réconfort moral. 


\section{Chapitre I}

LES ANNEIDES ET CERTAINES DE LEURS PROPRIETES ELEMENTAIRES

\section{$\S 1$ - NOTIONS D'ANNEIDE ET DE MODULOIDE.}

On appelle annélde tout ensemble non vide $A$ muni de deux lois internes: une addition, définie seulement pour certains couples dits addibles (notation \#), et une multiplication, vérifiant les axiomes :

A - Multiplication :

$$
\begin{aligned}
& 0-\forall a, b \in A, a b \in A \\
& 1-\forall a, b, c \in A,(a b) c=a(b c) \\
& 2 \text { - Il existe un élément } 0 \text { de } A, \text { tel que } 0 a=a 0=0, \forall a \in A .
\end{aligned}
$$

B - Addibilité :

$1-\forall a \in A, a \# a$

$2-\forall a, b \in A$, la relation $a \# b$ implique $b \# a$

$3-\forall a \in A, a \# 0$

$4-\forall a, b, c \in A$, les relations $a \# b, b \# c, b \neq 0$ impliquent $a \# c$. Pour $a \in A, a \neq 0$, notons $A(a)=\{x \in A ; x \# a\}$, appelé domaine

d'addibilité de $a$. Les axiomes $B_{3,4}$ montrent que $a \# b$ si et seulement s'ils appartiennent à un même domaine d'addibilité ; donc, deux tels domaines, ou sont confondus, ou ont en commun le seul elément 0 . On pose $D^{*}=\{A(a) ; a \in A, a \neq 0\}$ et $D=D^{*} \cup\{\{0\}\}$.

$C$ - Addition : Chaque domaine d'addibilité est un groupe additif abélien (dit groupe d'addibilite), c'est-à-dire que, pour tout $A(d) \in D^{*}$, on $a:$

$0-\forall a, b \in A(d), a+b \in A(d)$

$1-\forall a, b, c \in A(d),(a+b)+c=a+(b+c)$

$2-\forall a, b \in A(d), a+b=b+a$

3 - L'addition dans $A(d)$ possède un élément neutre

4 - Dans $A(d)$, tout élément possède un opposé.

( $L$ 'axiome $C_{0}$ équivaut a $C_{0}^{\prime}: \forall a, b \in A, a \# b$ implique $\left.a \# a+b\right)$.

D - Distributivité :

$\forall a, b, c \in A$, la relation $a$ \# $b$ implique :

$1-c a \# c b$

$2-c(a+b)=c a+c b$

$3-a c \# b c$

$4-(a+b) c=a c+b c$.

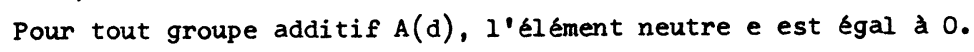
De $e+e=e$, on déduit $0(e+e)=0 e$, soit $0+0=0$. Ajoutons l'opposé $0^{\prime}$ de 0 dans $A(d): 0+\left(0+0^{\prime}\right)=0+0^{\prime}, d^{\prime}$ où $0+e=e$, soit encore $0=e$, car $e$ est neutre. 
L'annérde est dit commutatif s'il vérifie :

A $\quad 3-\forall a, b \in A, a b=b a$.

L'annétde est dit unitaire s'il vérifie :

A 4 - I un élément $1 \in A, 1 \neq 0$ tel que $1 \mathrm{a}=\mathrm{a} 1=\mathrm{a}, \forall \mathrm{a} \in \mathrm{A}$.

L'annérde est dit corporde si $A^{*}$ (A privé du 0 ) est un groupe multiplicatif, c'est-à-dire s'il vérifie $A$ et

A $\quad 5-\forall a \in A, a \neq 0$, I $a^{\prime} \in A$ tel que $a a^{\prime}=a^{\prime} a=1$.

Si de plus il est commutatif, il est dit corpolde commutatif.

Un annérde commutatif sans diviseur de zéro est dit intégrolde si

$A^{*}=A \ldots\{0\}$ est un semi-groupe (c'est-à-dire un monolde avec simplification : si $a \neq 0, a b=a c$ impliquent $b=c)$. Il est dit un intégrołde régulier si, en plus, $a \neq 0$ et $a b \neq a c$ impliquent $b \# c$.

Soit $A$ un annérde. On appellera modulołde à gauche sur $A$, ou

A-moduloyde + gauche, tout ensemble $M$ non vide muni d'une loi interne additive, définie seulement pour certains couples dits addibles (notation \#), et d'une loi externe partout définie, ayant A pour domaine d'opérateurs, notée multiplicativement, vérifiant les axiomes :

A - Multiplication :

$0-\forall a \in A, \forall x \in M, a x \in M$

$1-\forall a, b \in A, \forall x \in M,(a b) x=a(b x)$

2 - Il existe un élément $C^{\prime}$ de $M$ tel que, $\forall a \in A, \forall x \in M$, on ait a $O^{\prime}=0 x=0^{\prime}$. (On le notera désormais 0 ).

$B$ - Addibilité :

$1-\forall x \in M, x \not x$

$2-\forall x, y \in M$, la relation $x \not y$ implique $y \not x$

$3-\forall x \in M, x \not 0$

$4-\forall x, y, z \in M$, les relations $x \not y, y \# z, y \neq 0$ impliquent $x \# z$. De même que plus haut, on notera pour $x \in M, x \neq 0, \operatorname{par} M(x)$ le domaine d'addibilité de $x$. On pose de même :

$$
C^{*}=\{M(x) ; x \in M, x \neq 0\} \text { et } C=C^{*} \cup\{\{0\}\} \text {. }
$$

$C$ - Addition : Chaque domaine d'addibilité est un groupe additif abélien (dit groupe d'addibilité), c'est-à-dire que, pour tout $M(u) \in C^{*}$, on a :

$0-\forall x, y \in M(u), \quad x+y \in M(u)$

$1-\forall x, y, z \in M(u),(x+y)+z=x+(y+z)$

$2-\forall x, y \in M(u), \quad x+y=y+x$

3 - L'addition dans $M(u)$ possède un élément neutre

4 - Dans $M(u)$, tout élément possède un opposé.

( $L$ 'axiome $C_{0}$ équivaut à $C^{\prime}{ }_{0}: \forall x, y \in M$, la condition $x \not y$ implique $x \# x+y$ ). 
D - Distributivité :

$$
\begin{array}{r}
\forall a, b \in A, \forall x, y \in M: \\
1-x \# y \text { implique } a x \# a y \\
2-x \# y \text { implique } a(x+y)=a x+a y \\
3-a \# b \text { implique } a x \# b x \\
4-a \# b \text { implique }(a+b) x=a x+b x .
\end{array}
$$

On voit aisément que l'élément neutre de tout groupe additif $M(u)$ est

$0 \in M$. Les notions de moduloide à droite sur A (modifier l'écriture du produit et l'axiome $A_{1}$ ), et de moduloyde unitaire (lorsque $A$ est unitaire), sont les analogues du cas des modules.

\section{Propriété fondamentale :}

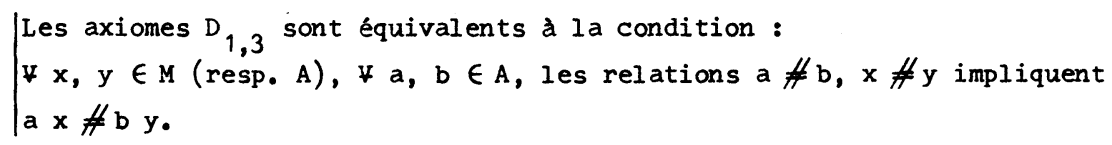

Si a \#b et $x \# y$, on a par distributivité :

a $x \# b x, b \times \# b$ y et $a x \# a y, a y \# b y$.

Si $b x \neq 0$, ou si a $y \neq 0$, la transitivité montre que $a x \not b y$.

Si $b x=0$ et a $y=0$, on a par distributivité

$(a+b) x \#(a+b) y d \cdot o u a x+0 \# 0+b y$.

La réciproque est évidente. Noter la conséquence suivante :

Si $a$ et $b \in A$ sont tels que $A(a) A(b) \neq\{0\}$, il existe un et un seul $A(c) \in D^{*}$ tel que $A(a) A(b) \subset A(c)$.

De même, si $a \in A$ et $x \in M$ sont tels que $A(a) M(x) \neq\{0\}$, il existe un et un seul $M(y) \in C^{*}$ tel que $A(a) M(x) \subset M(y)$.

Remarques de calcul :

Dans A, l'écriture $a_{1}+\ldots+a_{r}=\sum_{i=1}^{r} a_{i}$ suppose les $a_{i}$ addibles

deux à deux. De même, l'écriture $\left(a_{1}+\ldots+a_{r}\right)^{i=1}+\left(b_{1}+\ldots+b_{s}\right)$ suppose que chaque somme entre parenthèses a le sens précédent, et que les totaux sont addibles. Par exemple, si a et $b$ ne sont pas addibles, $a+(b-b)$ signifie $a+0=a$, mais $a+b-b n^{\prime} a$ pas de sens dans $A$.

Lemme : Soient deux sommes (d'éléments addibles) de total non nul : $\mid \begin{aligned} & \sum_{i=1}^{r} a_{i}=a \neq 0 \text { et } \sum_{j=1}^{s} b_{j}=b \neq 0 . \\ & s i a \# b, \forall i \text { et } j \text { on } a a_{i} \# b_{j} .\end{aligned}$

En effet, l'axiome $C_{0}$ montre que $a_{i} \# a, b_{j} \# b$, d'où le résultat par transitivité par a et b. On a la règle : Après avoir remplacé par leur valeur 0 les parenthèses de total nul, on a donc le droit de "supprimer les 
parenthèses".

Soient $A$ un annélde, $M$ un A-modulolde à gauche, $B$ et $B^{\prime}$ deux parties non vides de $A, X$ et $Y$ deux parties non vides de $M$. On notera : $X+Y$ l'ensemble des éléments de $M$ de la forme $x+y$, avec $x \in X, y \in Y$ et $x \# y$; B X I'ensemble des éléments de $M$ de la forme $\sum_{i=1}^{n} b_{i} x_{i}$ où $\forall i, b_{i} \in B, x_{i} \in X$ et les $b_{i} x_{i}$ addibles deux à deux ; $B x=B X$ lorsque $X=\{x\}$; enfin $B+B^{\prime}$ et $B^{\prime} B^{\prime}$ en considérant $A$ comme modulorde sur lui-même.

In élément de $\mathrm{B} X$ (resp. de $\mathrm{B} \mathrm{B}^{\prime}$ ) s'appelle une combinaison linéaire $\mathrm{d}^{\prime}$ éléments de $\mathrm{X}$ (resp. de $\mathrm{B}^{\prime}$ ) sur $\mathrm{B}$. Lorsqu'on prend pour $\mathrm{B} \mathrm{I}^{\prime}$ annéIde $A$, on dit combinaison linéaire $d^{\prime e ́ l e ́ m e n t s ~ d e ~} X$ (resp. $B^{\prime}$ ). Il faut bien remarquer $q u^{\prime} u n$ même élément $x_{i} \in X$ peut intervenir plusieurs fois dans une somme $\sum_{i=1}^{n} b_{i} x_{i}$, avec des éléments $b_{i}$ non forcément addibles. On pourra éventuellement souligner ce fait en notant un élément de B X par

$$
\sum_{j=1}^{m}\left(\sum_{k=1}^{r_{j}} b_{j}^{k} x_{j}\right)=\sum_{j=1}^{m} \beta_{j} x_{j}
$$

où les $x_{j}$ sont tous distincts et où $\beta_{j}$ est la somme formelle

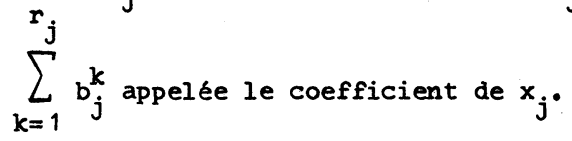

$\$ 2$ - ANNEAUX ET MODULES ASSOCIES. SOUS-ANNEIDES ET SOUS-MODULOIDES.

$$
\text { Notons } \bar{A}=A(a) \stackrel{\oplus}{\in} D^{*} A(a) \quad\left(\text { resp. } \bar{M}=M(x) \stackrel{\oplus}{\in} C^{*} M(x)\right. \text { ) }
$$

le groupe somme directe des groupes additifs $A(a)$ (resp. $M(x)$ ). Organisons $\bar{A}$ en anneau (resp. $\bar{M}$ en $\bar{A}$-module à gauche) en étendant la loi multiplicative de $A$ (resp. de M) par distributivité et réduction des termes semblables (c'est-à-dire appartenant à une même composante). Les propriétés d'associativité et de distributivité (et éventuellement la commutativité dans le cas de $\bar{A}$ ) sont évidentes; de même, si $A$ (resp. $M$ ) est unitaire, $\bar{A}$ (resp. $\bar{M}$ ) l'est aussi. $\bar{A}$ s'appelle I'anneau de I'annélde $A . \bar{M} s^{\prime}$ appelle le $\bar{A}$-module du A-modulorde $M$. Il est clair que $\bar{A}$ et $\bar{M}$ sont des $A$-modulotdes. Par contre, $A$ (resp. $M$ ) n'est pas, en général, un $\bar{A}$-modulotde, le produit n'étant pas forcément partout défini. $\bar{A}$ (resp. $\bar{M}$ ) sera dit anneau (resp. module) gradué généralisé.

$B$ est dit un sous-annelde de $A$, et $A$ un sur-annelde (ou une extension) de $B$ si l'addibilité et les lois de $A$ induisent sur $B$ une structure d'annétde, c'est-à-dire si on a (outre $B \subset A$ ) : 
1 - L'addibilité de $B$ est la restriction à $B$ de celle de $A$.

$2-\forall a \in A^{*}, B \cap A(a)$ est sous-groupe additif de $A(a)$.

$3-B^{2} \subset B$.

$N$ est dit un sous-moduloide de $M$, et $M$ un sur-modulorde de $N$ si

l'addibilité et les lois de $M$ induisent sur $N$ une structure de moduloide sur le

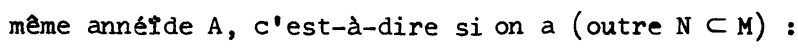

1 - L'addibilité de $N$ est la restriction à $N$ de celle de $M$.

$2-\forall x \in M^{*}, N \cap M(x)$ est un sous-groupe additif de $M(x)$.

$3-\mathrm{A} N \subset \mathrm{N}$.

La condition $A N \subset N$ équitvaut a $: \forall a \in A, \forall x \in N$, on a a $x \in N$.

II en est de même pour $B^{2} \subset B$.

Un sous-modulotde du A-moduloide à gauche A (resp. à droite) est dit idéal à gauche (resp. à droite) de A. On appelle idéal bilatère un idéal à gauche et à droite de A. Lorsque A est commutatif, on dit simplement idéal.

$\bar{A}$ étant un anneau gradué généralisé dont la partie homogène est un annérde $A$, un sous-anneau $S$ de $\bar{A}$ est dit homogène s'il est de la forme $\bar{B}$, où $B$ est un sous-annélde de A. Visiblement, ceci équivaut à l'égalité

$S=A(a) \stackrel{\oplus}{\epsilon} D^{*}[S \cap A(a)]$, autrement dit à la condition: si $\bar{a}=\sum_{i} a_{i}$

est la décomposition $d^{\prime} u n \bar{a} \in S$ en composantes homogènes, tous les $a_{i}$ sont dans S. Par ailleurs, si $B$ est un sous-annélde de $A, \bar{B}$ est un anneau, donc un sous-anneau de $\bar{A}$. Si un idéal (à droite, à gauche, ou bilatère) de $\mathrm{A}$ en est un sous-anneau homogène, il en est dit idéal hon igène.

De même, un sous-module d'un $\bar{A}$-modul $\bar{M}$, dont la partie homogène est $M$, est dit homogène $s^{\prime} i l$ est de la forme $\bar{N}$, où $N$ est un sous-modulotde de $M$. (si $N$ l'est, $\bar{N}$ est un $\bar{A}$-module, donc un sous-module de $\bar{M}$ ). Il est évident qu'un sousmodule $P$ du $\bar{A}$-module $\bar{M}$ est homogène si, et seulement $s^{\prime} i l$ contient avec un élément de $\bar{M}$ toutes ses composantes homojé.les. Visiblement, si $B$ est un sousannérde de $A$, le sous-anneau homogène $\bar{B}$ de $\bar{A}$ en est un idéal (forcément homogène) si et seulement si $B$ en est un de $A$.

Un annéfde $A$ et $I$ 'anneau gradué $\bar{A}$ sont dits annérde [resp. anneau gradué] noethérien (resp. artinien) à gauche ou à droite si, respectivement, les iléaux à gauche ou à droite de $\mathrm{A}$ (ou, ce qui revient au même, de tels idéaux homogènes de $\bar{A}$ ) satisfont à la condition maximale (resp. minimale). Il sera dit noethérien ou artinien touc court s'il l'est à la fois à droite et à gauche. Exemples d'annéides :

$\left.1^{\circ}\right)$ Le squelette d'un corps valué est un corpolde. Si q est un corpoide "sans torsion" et $n \geq 1$ un entier donnés, Krasner [4] définit les annéldes de polynômes à $n$ indéterminées sur $q$ (sous certaines conditions). 
$\left.2^{\circ}\right)$ Nous appellerons annétde de Bourbaki la partie homogène d'un anneau gradué de Bourbaki [1]. Citons deux cas particuliers :

Soient $k$ un corps, et $\left(x_{i}\right)_{i} \in I$ une famille d'indéterminées ; $I^{\prime}$ ensemble $Q$ des fractions monomiales par rapport aux $x_{i}$ à coefficients dans $K$, est un corporde. La partie A formée des monômes en est un sous-annérde.

Soient $R$ un anneau intègre (commutatif, avec unité) et $G=\{\varepsilon, \sigma\}$ un groupe à deux éléments $\left(\sigma^{2}=\varepsilon\right)$. Soit $\bar{A}=R \varepsilon+R \sigma l$ 'anneau gradué construit sur $R$ et $G$ de manière évidente. Soit $A I^{\prime}$ annélde associé. Alors, A est sans diviseur de 0 , mais $\overline{\mathrm{A}} \mathrm{n}^{\prime}$ est pas intègre : $(\sigma-\varepsilon)(\sigma+\varepsilon)=0$.

3)Plus généralement, soient $\Delta$ un monorde multiplicatif, commutatif et muni d'un annulateur (ou élément absorbant) noté $O$. Soit $R$ un anneau commutatif. Soit $\left(A_{\alpha}\right)_{\alpha \in \Delta}$ une famille d'anneaux tels que : $\forall \alpha \in \Delta, A_{\alpha}$ est isomorphe à un sous-anneau $R_{\alpha}$ de $R ; R_{0}=\{0\}$; et, $\forall \alpha$ et $B \in \Delta, R_{\alpha} R_{\beta} \subset R_{\alpha \beta^{*}}$ Prenons pour ensemble d'homogénérté la partıe $\mathrm{A}=\underset{\alpha \in \Delta}{U} \mathrm{~A}_{\alpha}$ de $\overline{\mathrm{A}}=\underset{\alpha \in \Delta}{\oplus} \mathrm{A}_{\alpha}$. Si $x_{\alpha}$ et $y_{\beta}$ sont des éléments de $A_{\alpha}$ et $A_{\beta}$ respectivement, par définition, leur produit sera 1 'élément de $A_{\alpha \beta}$ homoloque du produit $x y$ dans $R$ des homologues $x$ $\left(\right.$ de $\left.x_{\alpha}\right)$ et $y\left(\right.$ de $\left.y_{\beta}\right)$. Alors, il est clair que $A$ est un annérde.

$\mathrm{Si}$, de plus, $\mathrm{R}$ et $\Delta$ ont un élément unité, respectivement 1 et $\boldsymbol{\varepsilon}$, la condition $1 \in R_{\varepsilon}$ entraine que 1 est unité de $A$.

$\$ 3$ - LA NOTION DE GRADUATION.

On va définir sur $D$ une loi de composition partielle. Afin de pouvoir l'écrire sans introduire de nouveau signe d'opération tout en distinguant le composé des éléments de $D$ selon cette loi, de leur produit en tant que sous-ensembles de A (qui est différent, en général), nous introduisons l'abus d'écriture suivant : si a $\in A$ est non nul, son domaine d'addibilité sera noté $A(a)$ s'il est considéré comme sous-ensemble de $A$, et sera noté $\omega(a) s^{\prime i}$ l est considéré comme élément de $D^{*}$. (Quand il est indifférent de considérer ce domaine d'une manière ou de I'autre, on se permettra d'écrire $A(a)=\omega(a))$. De mème, $\{0\} \subset A$ sera, comme élément de $D$, noté $\omega(0)$ ou 0 . Pour tout a $\in A$, $\omega(\mathrm{a})$ sera dit son grade propre. Donc, $A(a) A(b)$ (ou $A(a)\{0\}$ ) désignera le produit de $A(a)$ et $A(b)$ (ou $\{0\}$ ) comme sous-ensembles de $A$, tandis que $\omega(\mathrm{a}) \omega(\mathrm{b})$ (ou $\omega(\mathrm{a}) \omega(0)=\omega(\mathrm{a})$ 0) désignera leur produit dans $D$ suivant la loi partielle ainsi définie :

(L)

$$
\begin{aligned}
& -\forall \omega(a) \in D \text {, on pose } 0 \omega(a)=\omega(a) 0=0 \\
& -\forall \omega(a) \text { et } \omega(b) \in D^{*} \text {, tels que } A(a) A(b) \neq\{0\} \text {, on sait qu'il existe } \\
& \text { un unique } A(c) \in D^{*} \text { tel que } A(a) A(b) \subset A(c) \text { et on pose } \\
& w(a) \omega(b)=\omega(c) \text {. }
\end{aligned}
$$


Le produit $\omega(a) \omega(b)$ n'est pas défini quand $a \neq 0, b \neq 0$ et $A(a) A(b)=\{0\}$.

On appellera une graduation de l'annérde A tout couple $(\Delta, \theta)$ (qu'on notera simplement $\Delta$ quand il $n^{\prime} y$ aura pas d'ambiguité sur $\theta$ ), ou $\Delta$ est un ensemble muni d'une loi de composition (notée multiplicativement) partout définie et comportant un annulateur (noté 0 ), et où $\theta: \Delta \rightarrow D$ est une surjection, qui est injective sur l'image réciproque $\theta^{-1}\left(D^{*}\right)$ de $D^{*}$ par $\theta$, et qui vérifie les conditions : $\theta(0)=0$; et, $\forall \alpha, \beta \in \Delta$, on a $\theta(\alpha \beta)=\theta(\alpha) \theta(\beta)$ si $\theta(\alpha) \neq 0, \theta(\beta) \neq 0$ et $\theta(\alpha) \theta(\beta)$ est defini.

Le plus souvent, la restriction injective de $\theta$ à $\theta^{-1}\left(D^{*}\right)$ sera considérée comme une identification, et on identifie aussi l'annulateur 0 de $\Delta$ avec l'élément $\omega(0)=0 \in D$. Sauf mention contraire, on supposera donc que $\Delta \supset D$. Si $d=\omega(a)$, en vertu de I'identification précédente (c'est-à-dire si $\theta(d) \neq 0$ ou si $d=0$ ), d sera dit le grade de a dans $\Delta$. Un $d \in \Delta$ sera dit un grade nul par rapport à $A$ si $\theta(d)=0$, et il sera dit vide dans $A$ si, de plus, $d \neq 0$. Si $\theta(d)=\omega(a) \in D$, le même élément de $D$ considéré comme sous-ensemble de $A$ sera aussi noté $A_{d}$.

On appellera graduation propre de $A$ (et de $\bar{A}$ ) la graduation $A_{0}$ de support $D$, où on étend la loi partielle (L) à $D$ tout entier en posant $\omega(a) \omega(b)=\omega(0)=0$, quand $a \neq 0, b \neq 0$ et $A(a) A(b)=\{0\}$.

Bien entendu, on a des considérations analogues pour l'ensemble C d'un A-modulorde à gauche $M$. En usant de l'abus d'écriture analogue, on définira dans C la loi partielle de composition externe : $\mid \begin{aligned} & \forall \alpha \in D, \forall \xi \in C \text { on pose } \alpha 0=0 \xi=0 . \\ & \forall \omega(a) \in D, \forall \omega(x) \in C \text {, } a \neq 0, x \neq 0 \text { tels que } A(a) M(x) \neq\{0\} \text {, donc } \\ & \text { est contenu dans un unique } M(y) \text {, on pose } \omega(a) \omega(x)=\omega(y) .\end{aligned}$

Cette loi n'est pas forcément partout définie, ni associative pour les scalaires (ou éléments de $D$ ).

Lorsqu'est fixée une graduation $(\Delta, \theta)$ de $A$, on appelle une $\Delta$-graduation du A-modulorde $M$, tout couple $(\Gamma, \psi)$ formé d'un ensemble $\Gamma$ sur lequel $\Delta$ opère multiplicativement à gauche, avec annulateur noté 0 , et d'une surjection $\psi: \Gamma \rightarrow C$, dont la restriction à $\psi^{-1}\left(C^{*}\right)$ est injective, avec les conditions : $\forall(0)=0 ; \forall d \in \Delta, \forall c \in \Gamma$, on a $d_{0} 0=0 . c=0$; $\forall a \in \Delta$ et $\forall c \in \Gamma$ tels que $\theta(d) \neq 0, \forall(c) \neq 0$ et $\theta(d) \forall(c)$ soit défini en vertu de $\left(L^{\prime}\right)$, on $a \psi(d c)=\theta(d) \psi(c)$. Si $\psi(c)=\omega(x) \in C$, cet elément de $C$ considéré comme sous-ensemble de $M$, sera noté $M_{C}$. On pourra considérer la restriction de $\psi^{-1}\left(C^{*}\right)$ comme une identification, et identifier les zéros

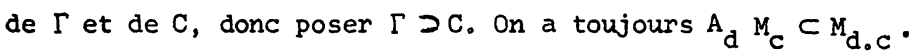


Par exemple, on appellera $\Delta$-graduation propre de $M$ la graduation $\Gamma_{0}$ de support $C$ où on étend la loi partielle $\left(L^{\prime}\right)$ en posant d. $\omega(x)=\omega(0)=0$ quand $d \neq 0, x \neq 0$ et $A_{d} M(x)=\{0\}$.

$L^{\prime}$ exemple 1 suivant montre qu'on ne peut pas forcément étendre (L) à $D$ tout entier pour en faire un monoide. Mais, parmi toutes les graduations possibles pour un annérde donné $A$, en existe-t-il toujours une associative, c'est-à-dire (au cas où A serait unitaire), pour laquelle l'anneau $\bar{A}$ serait gradué au sens Bourbaki ? Existe-t-il une extension $A^{\prime}$ de $A$, ayant ou non le même ensemble $D$, et telle que tout groupe d'addibilité différent de $\{0\}$ contienne un non-diviseur de 0 ? Sans hypothèse supplémentaire, la réponse est partout négative, comme le montre 1 'exemple 2 suivant.

De même, lorsque $\Delta$ est une graduation associative de $A$, une $\Delta$-graduation $\Gamma$ de $M$ sera dite associative si sa loi vérifie l'associativité des scalaires (ou éléments de $\Delta$ ). Un exemple ultérieur montrera l'existence de $\Delta$-graduations non associatives (chap. II, $\S 3$ ).

Si $A=q$ est un corpolde, $D^{*}$ est visiblement un groupe par rapport à la loi de composition de $\Delta_{0}$. De plus, $R=q(1)$ est un corps (gauche, en général) car $R^{*}=R \ldots\{0\}$ est un sous-groupe de $q^{*}=q \ldots\{0\}$. Si a $\in$ q est non nul, $\sigma: R \rightarrow q(a)$ défini par $\sigma(x)=a x$, et $\tau: q(a) \rightarrow R$ défini par $\tau(y)=a^{-1} y$ sont des homomorphismes de groupes additifs, dont les composés sont des identités. Ce sont donc des isomorphismes, et $q$ (a) est un espace vectoriel bilatère de dimension 1 sur $R \cdot D^{*}=\Delta_{0}^{*}$ est dit le groupe du corpolde $q$, et q est dit sans torsion si le groupe $D^{*} I^{\prime}$ est. $K=q(1)$ est dit le corps du corpoide.

$\mathrm{Si} \mathrm{A}$ est un intégroł́de régulier, $D^{*}$ est visiblement un monolde commutatif avec simplification, par rapport à la loi de $\Delta_{0}$.

Exemple 1 : Soient $R$ un anneau commutatif donné, $R^{\prime}$ et $R^{\prime \prime}$ deux anneaux isomorphes à $R$, deux d'entre eux ayant en commun le seul élément 0 . Soient les anneaux produits $A_{\varepsilon}=R \times R, A_{a}=R^{\prime} \times(0)$ et $A_{b}=(0) \times R^{\prime \prime}$. Organisons la réunion $A=A_{\varepsilon} \cup A_{a} \cup A_{b}$ de leurs groupes respectifs en annélde en posant pour le produit (les accents des composantes indiquant seulement 1'appartenance des éléments) commutatif : $\left(x_{1}, y_{1}\right)\left(x_{2}, y_{2}\right)=\left(x_{1} x_{2}, y_{1} y_{2}\right) ;\left(x_{1}, y_{1}\right)\left(x_{2}^{\prime}, 0\right)=\left[\left(x_{1} x_{2}\right)^{\prime}, 0\right]$. $\left(x_{1}, y_{1}\right)\left(0, y_{2}^{\prime \prime}\right)=\left[0,\left(y_{1} y_{2}\right)^{\prime \prime}\right] ;\left(x^{\prime}, 0\right)\left(y^{\prime}, 0\right)=\left[0,(x y)^{\prime \prime}\right]$. $\left(0, x^{\prime \prime}\right)\left(0, y^{\prime \prime}\right)=(0, x y) ;\left(x^{\prime}, 0\right)\left(0, y^{\prime \prime}\right)=(0,0)$ 。

Sur l'ensemble $D$, noté $\{0, \varepsilon, a, b\}$, correspondant, la loi (L) n'est point partout définie : $\varepsilon$ est unité, $a^{2}=b$ et $b^{2}=\varepsilon$; mais ab et ba ne sont pas définis, et $l^{\prime}$ on ne peut étendre ( $L$ ) a $D$ tout entier pour en faire un monorde : 
si on prend $a b=a$, on $a \quad \varepsilon=a^{2} b \neq a(a b)=b$

$$
\begin{array}{ll}
a b=b, & a=a b^{2} \neq(a b) b=\varepsilon \\
a b=\varepsilon, & a=a b^{2} \neq(a b) b=b \\
a b=0, & a=a b^{2} \neq(a b) b=0 .
\end{array}
$$

Cependant, $\Delta=D \cup\left\{c=a^{3}\right\}=\left\{0, \varepsilon, a, a^{2}, a^{3}\right\}$ est évidemment une graduation associative de $A$, puisque $\left\{\varepsilon, a, a^{2}, a^{3}\right\}$ est le groupe cyclique à quatre éléments engendré par a. Il suffit de poser $A_{a^{2}}=A_{b}$ et $A_{a^{3}}=\{0\}$, en prenant les mêmes $A_{\varepsilon}, A_{a}$ et $A_{b}$ que précédemment.

Exemple 2 : Soit $n$ un entier $\geq 3$. Soit $E=\left\{e, \ldots, e_{n}\right\}$ un ensemble de $n$ éléments muni d'une loi interne partout définie, commutative, et notée multiplicativement (cette loi donnée $n$ 'est pas forcément associative). Soit $R$ un anneau intègre (commutatif et unitaire). Soit $s=\underset{i \in \mathbb{Z}}{\oplus} R_{i}$ où, $\forall i \in \mathbb{Z}$, on prend pour $R_{i}$ le groupe additif de $R_{\text {. }}$

(10) A chaque $e_{k} \in E$, faisons correspondre une partie finie $P_{k} \subset \mathbb{Z}$, ainsi définie :
a) $-(2 k-1) \in P_{k}$

b) On répartit dans les $P_{k}$ les $\frac{n(n-1)}{2}$ premiers entiers impairs positifs, chacun deux fois, et de manière que :

- chaque $P_{k}$ contienne ( $\left.n-1\right)$ impairs distincts

- si $k \neq k^{\prime}, P_{k}$ et $P_{k^{\prime}} n^{\prime}$ ont en commun qu'un seul impair positif, noté $i_{k, k} \cdot$ •

c) On répartit les doubles des entiers impairs précédents, qui sont au

nombre de $n+\frac{n(n-1)}{2}=\frac{n(n+1)}{2}$ :

si $e_{k}^{2}=e_{k^{\prime \prime}}$ on pose $-2(\bar{k}-1) \in P_{k "}$

si $k \neq k^{\prime}$ et $e_{k} e_{k^{\prime}}=e_{k^{\prime \prime}}$ on pose $2 i_{k, k^{\prime}} \in P_{k^{\prime \prime}}$.

Chacun de ces nombres pairs ne figure que dans un seul ensemble.

d) Les $P_{k}$ ne contiennent pas d'autre entier.

(2•) a) A chaque $P_{k}$ (donc a chaque $\alpha_{k}$ ), on associe un groupe $A_{k}$ isomorphe au sous-groupe additif suivant du groupe abélien $S: A_{k} \approx \underset{i \in P_{k}}{\oplus} R_{i}$.

Soit $A=\bigcup_{k=1}^{n} A_{k}$ la partie du groupe somme directe $\bar{A}=\underset{k=1}{\bigoplus} A_{k}$.

b) Definition d'un produit dans $\mathrm{A}$; outre le cas trivial où l'un des facteurs est nul, distinguons deux cas :

Si $x \in A_{k}$ et $y \in A_{k}$, avec $k \neq k^{\prime}$. Le produit, élément $z$ de $A_{k \prime \prime}$ ( $s i$ $\left.e_{k} e_{k^{\prime}}=e_{k^{\prime \prime}}\right)$, se fera par composante, en doublant les indices des composantes. Plus précisément, seules les composantes d'indice $i_{k, k}$, de $x$ et y peuvent être toutes deux non nulles: 
$x=\left(\ldots \ldots \ldots, x_{i_{k, k}}, \ldots \ldots\right) ; y=\left(\ldots \ldots, y_{\left.i_{k, k}, \ldots . .\right)}, \ldots\right.$,

On pose $x y=z=\left(\ldots \ldots, z_{2 i_{k, k^{\prime}}}, \ldots ..\right)$ avec $z_{2 i_{k, k^{\prime}}}=x_{i_{k, k^{\prime}}} y_{i_{k, k^{\prime}}}$

Si $x$ et $y \in A_{k}$, le produit $z$, élément de $A_{k "}\left(s i e_{k}^{2}=e_{k^{\prime \prime}}\right)$, se fera par composante, en doublant les indices, mais en ne conservant que la composante d'indice - $2(2 k-1)$ négatif, et en annulant les autres.

c) Ce produit fait de A un annérde commutatif de manière évidente (en particulier, l'associativité du produit est triviale, tout produit de trois termes étant nul).

$\left(3^{\circ}\right) \forall k$ et $k^{\prime}, A_{k} A_{k^{\prime}} \neq(0)$, puisque $I^{\prime}$ anneau $R$ est intègre, et $A_{k} A_{k^{\prime}} \subset A_{k^{\prime \prime}}$ (notations $\left.d u 1^{\circ}\right)$. E est famille d'indices des $A_{k}$. Le produit (L) est défini partout sur $E U\{0\}=\Delta$ et coincide sur $E$ avec la loi de E (par construction).

Si la loi donnée sur $\mathrm{E}$ est non-associative, la graduation $\Delta$ de $\mathrm{A}$ sera non-associative, et toute autre graduation de $A$ ne sera pas associative.

Remarque : La donnée de la loi sur E équivaut à la donnée de la

répartition $(10-c)$ des $\frac{n(n-1)}{2}$ entiers pairs.

Remarque : Montrons, par récurrence sur $n$, que la répartition $\left(1^{0}-b\right)$ des entiers impairs est possible :

- si $\mathrm{n}=3$, on prend $\mathrm{A}_{1}=\{-1,1,3\}$

$$
\begin{aligned}
& A_{2}=\{-3,1,5\} \\
& A_{3}=\{-5,3,5\} .
\end{aligned}
$$

- si la répartition est faite pour l'entier $n$, il reste, pour l'entier $\mathrm{n}+1$, à répartir deux fois les $\mathrm{n}$ entiers impairs consécutits suivants : $2 n(n-1)+1, \ldots \ldots \ldots .$. . On les attribue une premiere fois tous a $A_{n+1}$, puis une deuxième fois en en donnant un et un seul (arbitrairement) à chacun des $A_{1}, \ldots, A_{n}$. $\S 4$ - QUOTIENTS - MORPHISMES.

Désormais, A désigne un annérdc commutatif.

Définition : Soit $\Im$ un idéal de A. Deux éléments a et $\mathrm{b}$ de $\mathrm{A}$ sont dits congrus modulo $\Im$ (on note $a \equiv b(\Im)$, ou même $a \equiv b^{\prime} s^{\prime} i l$ n'y a pas d'ambiguité) si et seulement si, ou bien a et $b \in \Im$, ou bien a $\notin \Im$, mais $a$ a $b$ et $a-b \in \Im$.

Il est clair que c'est une relation d'équivalence sur $\mathrm{A}:$ en particulicr, si $a \equiv b, b \equiv c$ et $b \notin\}$, on $a: b \neq 0$, $a \# b$ et $b \# c$, ce qui implique $a, b$ et $c$ addibles, d'où $a-c=(a-b)+(b-c) \in \Im$. Notons que, dans chaque groupe d'addibilité $A_{\alpha}$, cette congruence se réduit à la congruence modulo le sous-groupe $\mathfrak{\Im}_{\alpha}=\Im \cap \mathrm{A}_{\alpha}$. Les classes d'équivalence sont donc $\Im_{\text {, }}$ 
et les classes $a+\Im$ distinctes, avec a $\in \mathrm{A}$, a $\notin \Im$ (pour chacune de ces dernières, noter que $a+\Im \subset A(a))$. Ainsi, on voit que cette congruence est la relation $R$ d'équivalence la plus fine telle que $a \not b b$ et $a-b \in \Im$ impliquent $a \mathrm{R}$ b.

Lemme : Pour qu'une relation d'équivalence $R$ sur $A$ soit une congruence modulo un idéal $\Im$, il faut et il suffit qu'elle vérifie les conditions : $\forall a, b, a^{\prime}$ et $b^{\prime} \in A$,

1) $a R b$ et non $a R O$ impliquent $a \not \# b$

2) $a R b, a^{\prime} R b^{\prime}$, $a \# a^{\prime}$ et $b \# b^{\prime}$ impliquent $\left(a+a^{\prime}\right) R\left(b+b^{\prime}\right)$

3) $a R b$ et $a^{\prime} R b^{\prime}$ impliquent $\left(a a^{\prime}\right) R\left(b b^{\prime}\right)$.

On dit alors que $R$ est normale pour l'addibilité, et compatible avec la somme et le produit dans A. La congruence modulo $\Im$ vérifie ces conditions ; c'est clair pour 1. Pour 2, c'est clair si a, b, a' et $b^{\prime} \in \mathcal{I}$; si $a \notin \Im, a \equiv b$ implique $a \# b, a-b \in \Im$ et $b \notin \Im$, d'où par transitivité (puisque $a$ et $b$ non nuls) $a, b$, $a^{\prime}$ et $b^{\prime}$ addibles entre eux, donc $a^{\prime}-b^{\prime} \in \Im$ et enfin $\left(a+a^{\prime}\right)-\left(b+b^{\prime}\right)=(a-b)+\left(a^{\prime}-b^{\prime}\right) \in 9$. Pour 3, c'est clair si $a \in \Im\left(d^{\prime}\right.$ ou $\left.b \in \Im\right)$ ou $a^{\prime} \in \Im$, car les produits sont dans $\Im$; si aucun des éléments $a, b, a^{\prime}$ et $b^{\prime} n^{\prime}$ est dans $\Im$, on $a$ a \#b, $a-b \in \mathcal{G}, a^{\prime} \# b^{\prime}$ et $a^{\prime}-b^{\prime} \in \Im$; par la propriété fondamentale, $a a^{\prime}, b^{\prime} b^{\prime}$ et $a b^{\prime}$ sont addibles entre eux, d'où : $a a^{\prime}-b b^{\prime}=a a^{\prime}-a b^{\prime}+a b^{\prime}-a^{\prime} b^{\prime}=a\left(a^{\prime}-b^{\prime}\right)+\left(a-a^{\prime}\right) b^{\prime} \in \Im$. Inversement, si la relation $R$ d'équivalence vérifie ces conditions, $\Im=\{a \in A ; a R 0\}$ est un idéal de $A: 0 \in \Im$; si a et $b \in \Im$ sont tels que $a \# b, b R O$ entraine - $b R O$, qui, avec a $R 0$ entratne $(a-b) R 0$ (tout ceci par la condition 2); si $a \in A$ (donc a $R$ a), et $\times R 0,1 a$ condition 3 implique a $\times \mathrm{R} 0$. Montrons que $\mathrm{R}$ est la congruence modulo $\Im$. Lorsque a $R 0$, soit $a \in \Im$, les relations suivantes sont équivalentes : a $R$ b, (par transitivité de $R$ ) b R $0, b \in \Im, a \equiv b$. Lorsque $a \in \Im$, si a $R$ b ou si $a \equiv b$, on $a$ a \#b par la condition 1 : les relations suivantes sont donc équivalentes : a $R \mathrm{~b},($ par la condition 2) ( $a-b) R 0, a-b \in \Im_{1} a \equiv b$. $L$ 'ensemble quotient $A / \Im$ est muni canoniquement d'une structure d'annérde : Notons $\varphi: A \rightarrow A / \Im^{l}$ la surjection canonique. Si $\alpha$ et $\alpha^{\prime} \in \mathrm{A} / \mathfrak{I}$, soient a et $a^{\prime}$ des représentants respectifs de ces classes, on pose $\alpha \alpha^{\prime}=\varphi\left(a a^{\prime}\right)$, produit indépendant du choix de a et $a^{\prime}$, grace à la compatibilité (3); les axiomes A pour $A / \Im$ se vérifient trivialement (le zéro est $\Im$, et, si $1 \in \mathrm{A}, I^{\prime}$ unité est $\left.1+\Im\right)$. Deux classes $\alpha$ et $\alpha^{\prime}$ seront addibles si et seulement si elles contiennent un élément addible (soient a et a' respectivement); on pose alors $\alpha+\alpha^{\prime}=\varphi\left(a+a^{\prime}\right)$, somme indépendante du 
choix du couple d'addibles a et $a^{\prime}$, grâce à la compatibilité (2). Les axiomes B pour $\mathrm{A} / \mathrm{g}$ se vérifient aisément : pour $\mathrm{B}_{4}$, si $\alpha \# \beta, \beta \# \gamma, \beta \neq 0$, alors il existe $a \in \alpha, b$ et $b^{\prime} \in \beta, c \in \gamma$ tels que $a \not b, b^{\prime} \# c$; mais $\beta \neq 0$

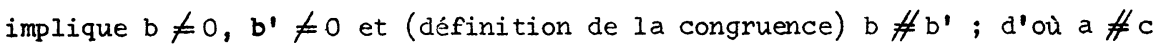
par transitivité par b et b'. Les axiomes pour l'addition sont clairs : Remarquer que chaque domaine d'addibilité de $\mathrm{A} / \mathfrak{\Im}$ est isomorphe au quotient d'un groupe $\mathrm{A}_{\alpha}$ d'addibilité de $\mathrm{A}$ par le sous-groupe $\mathfrak{J}_{\alpha}=\mathfrak{\Im} \cap \mathrm{A}_{\alpha} \cdot$ La distri-

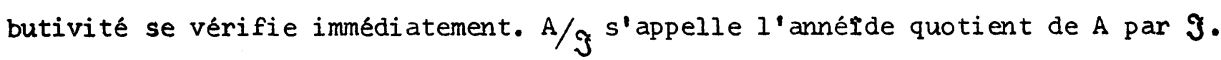
Remarquons que $\overline{(\mathrm{A} / \mathcal{J})}$ peut s'identifier à $I^{\prime}$ anneau quotient $\bar{A} / \bar{I}$ de I'anneau $\bar{A}$ par son idéal homogène $\overline{\mathfrak{J}}$, et que cette identification organise $\bar{A} / \sqrt{\mathfrak{S}}$ en anneau gradué dont la graduation est, en quelque sorte, induite par celle de $\mathrm{A}$ (résultat bien connu dans les cas classiques). En effet, si a $\rightarrow$ a' est l'homomorphisme canonique de $A$ sur $A / \Im$ et $\bar{a} \rightarrow \bar{a}^{n}$ celui de $\bar{A} \operatorname{sur} \bar{A} / \mathcal{I}$, on a pour tout $a \in A$, en considérant $a^{\prime}$ et $a^{\prime \prime}$ comme sous-ensembles de $\bar{A}$, $a^{\prime \prime}=a^{\prime}+\bar{S}$ et $a^{\prime}=a^{\prime \prime} \cap A$. Il en résulte que, si $a \not k b, a^{\prime \prime}+b^{\prime \prime}=(a+b) "=(a+b)^{\prime}+\overline{\mathcal{S}}=a^{\prime}+b^{\prime}+\overline{\mathfrak{S}}$ et que $a^{\prime \prime} b^{\prime \prime}=\left(\begin{array}{ll}a & b\end{array}\right)^{\prime \prime}=(a b)^{\prime}+\overline{\mathfrak{S}}=a^{\prime} b^{\prime}+\overline{\mathfrak{S}}$. Il en résulte facilement que si $\bar{a}=\sum_{i=1}^{s} a_{i}$ (les $a_{i}$ deux à deux non addibles), $: \sum_{i} a^{\prime}{ }_{i} \rightarrow \sum_{i} a^{\prime}{ }_{i}+\overline{\mathfrak{J}}=\bar{a}^{\prime \prime}$ définit un homomorphisme de $\overline{(A / \sqrt{S}}$ sur $\bar{A} / \bar{J}$. Mais $\bar{a}^{\prime \prime}=0$ entraine, puisque tous les $a^{\prime}{ }_{i}$ non nuls sont mutuellement non addibles comme le sont les $a_{i}$, que tous les $a^{\prime}{ }_{i}$ sont nuls et que $\sum_{i} a^{\prime}{ }_{i}=0$. Donc t est un isomorphisme. Soient $A$ un annéide, $M$ un A-moduloide, et $P$ un sous-moduloide de $M$. La notion de congruence modulo $P$ dans $M$ est $I^{\prime}$ analogue de la congruence modulo $\mathfrak{J}$ dans A. Les propriétés sont en tout point analogues, sauf pour la compatibilité (3) du lemme, que l'on remplace par la compatibilité avec le produit par un scalaire : ( $\left.3^{\prime}\right) \forall a \in A, \forall x$ et $y \in M, x R$ y implique a $x R$ a $y$. on notera indifféremment $M / P$ ou $M-P 1$ 'ensemble des classes de cette congruence modulo $P$. On notera $\varphi: M \rightarrow M-P$ la surjection canonique. Alors, l'ensemble $M$ - P est muni canoniquement d'une structure de A-moduloide : les mêmes considérations sont valables pour l'addibilité et l'addition ; enfin, si $a \in A$ et $\xi \in M-P$, on prend $x$ quelconque dans $\xi$, et on pose a $\xi=\varphi(a x)$, produit indépendant du choix de x par la compatibilité ( $\left.3^{\prime}\right)$; les propriétés sont immédiates. $M-P=M / P$ s'appelle le A-moduloide quotient de $M$ par $P$.

De la même manière que précédemment, on démontre $\overline{(M-P)}$ est canoniquement isomorphe au module quotient $\overline{\mathrm{A}}-\overline{\mathrm{P}}$ du $\overline{\mathrm{A}}$-module $\bar{M}$ par son sous-module $\overline{\mathrm{P}}$. Ceci montre, comme il est connu dans le cas classique, que 
$\bar{M}-\bar{P}$ peut être organisé en un $\bar{A}$-module gradué par la même graduation que $\bar{M}$. Soient $A$ et $B$ deux annérdes. Une application $u: A \rightarrow B$ est dite homomorphisme d'annérdes si, $\forall$ a et $b \in A$, on $a$ :

$$
\begin{aligned}
& 1-a \# b \text { implique } u(a) \# u(b) \\
& 11-u(a) \# u(b), u(a) \neq 0, u(b) \neq 0 \text { impliquent } a \# b \\
& 2-a \# b \text { implique } u(a+b)=u(a)+u(b) \\
& 3-u(a b)=u(a) u(b) . \\
& \quad \text { Si } l^{\prime} \text { axiome (1') n'est pas vérifié, on a une représentation (au }
\end{aligned}
$$
sens de Bourbaki) que nous appellerons quasi-morphisme, ou homomorphisme faible.

Si, de plus, A et $B$ sont unitaires, et si $u(1)=1$, u sera dit homomorphisme (resp. quasi-morphisme) d'annérdes unitaires. Il est clair que $u(0)=0$. Si $u$ est un quasi-morphisme ou un homomorphisme, on appelle noyau de $u$, et on note Ker $u=u^{-1}(0)$. Les axiomes $1,2,3$ montrent que le noyau est un idéal de $A$, la surjection canonique $\varphi: A \rightarrow A / \Im$ est un homomorphisme. Lorsque I'annérde $\mathrm{A} n$ 'est pas un anneau, I'injection canonique $A \rightarrow \bar{A}$ est seulement un quasi-morphisme.

Soient $M$ et M' $^{\prime}$ deux A-moduloides. Une application $u: M \rightarrow M^{\prime}$ est dite un homomorphisme de A-modulordes si, $\forall$ a $\in A$ et $\forall x$ et $y \in M$, on a :

$$
\begin{aligned}
& 1-x \not y \text { implique } u(x) \# u(y) \\
& 1 '-u(x) \not u(y), u(x) \neq 0, u(y) \neq 0 \text { impliquent } x \# y \\
& 2-x \not y \text { implique } u(x+y)=u(x)+u(y) \\
& 3-u(a x)=a u(x) .
\end{aligned}
$$

Si l'axiome ( $\left.1^{\prime}\right) n^{\prime}$ est pas vérifié, on dit encore quasi-morphisme. De même, on voit dans les deux cas que $u(0)=0$ et que Ker $u=u^{-1}(0)$ est un sous-A-modulorde de M.

Si $P$ est un sous-moduloide de M, l'injection canonique est un homomorphisme, de même que la surjection canonique $M \rightarrow M / P^{\bullet}$ L'injection canonique $M \rightarrow \bar{M}$ est un quasi-morphisme. Soit a fixé $\in A ; I$ 'homothétie $h$ de $M$ définie par $h(x)=a x$ est un quasi-morphisme.

Un homomorphisme ou un quasi-morphisme $u: A \rightarrow B$ d'annérdes (resp. $u: M \rightarrow N$ de $A$-modulołdes) se prolonge par linéarité, de manière évidente, en un homomorphisme $\bar{u}: \bar{A} \rightarrow \bar{B}$ d'anneaux gradués (resp. $\bar{u}: \bar{M} \rightarrow \bar{N}$ de $\bar{A}$-modules). Un homomorphisme $v: \bar{A} \rightarrow \bar{B}$ (resp. $v: \bar{M} \rightarrow \bar{N}$ ) sera dit homogène [resp. quasihomogène] si $v=\bar{u}$, où $u: A \rightarrow B$ (resp. $u: M \rightarrow N$ ) est un homomorphisme [resp. quasi-morphisme].

Les annétdes ou moduloides (respectivement les anneaux gradués ou leurs modules gradués) peuvent être considérés comme les classes d'objets de deux catégories différentes : la catégorie forte, en prenant pour morphismes les 
homomorphismes (resp. les homomorphismes homogènes), et la catégorie faible, en prenant comme morphismes les quasi-morphismes (resp. les homomorphismes quasihomogènes). $\mathrm{x} \rightarrow \overline{\mathrm{X}}$ et $\mathrm{u} \rightarrow \overline{\mathrm{u}}$ définit visiblement un isomorphisme de la catégorie, forte ou faible, des annéldes (resp. des A-moduloldes) avec la catégorie de même nom des anneaux gradués (resp. des $\overrightarrow{\mathrm{A}}$-modules gradués).

Une sous-catégorie de la catégorie forte ou faible des annéldes ou des $A$-moduloldes (et, également, son image par $x \rightarrow \bar{X}, u \rightarrow \bar{u}$ ) sera dite régulière si, quels que soient les objets $X, Y$ de la catégorie, et quel que soit $x$ fixé $\in X$, tous les $u(x)$, où $u$ parcourt Hom (X,Y) (dans la sous-catégorie), sont mutuellement addibles. Les sous-catégories régulières de la catégorie forte sont exactes, mais pas, en général, celles de la catégorie faible. 
Dans ce qui suit, les annérdes sont supposés commutatifs.

\section{$\S 1$ - LE PHENOMENE D'AGGLUTINATION.}

Pour un élément non nul $x$ d'un annérde $A$, les propriétés d'être ou non diviseur de zéro, ou simplifiable, ou diviseur d'un autre élément non nul sont les mêmes que dans les anneaux. Mais, pour un annérde dont les grades non nuls ne forment pas un groupe, la multiplication par un élément peut donner des résultats addibles à partir d'éléments qui ne le sont pas. Plus généralement, il peut en être de même dans l'application d'un quasi-morphisme, comme le montre l'injection canonique d'un annérde $\mathrm{A}$ dans $I^{\prime}$ anneau $\overline{\mathrm{A}}$ associé. Ce phénomène sera appelé l'agglutination. Il conduit au dédoublement de certaines notions habituelles (par exemple, diviseur et pseudo-diviseur), et à l'apparition de notions nouvelles. D'autre part, vue l'importance de la condition préalable d'addibilité dans la plupart des raisonnements, il parait souhaitable de réserver l'expression "régularité d'un élément", à la simplifiabilité dans une relation d'addibilité, avec un sens que l'on va préciser.

Soit $x$ un élément non nul d'un annérde A. $x$ est dit régulier pour l'addibilité (ou régulier, s'il n'y a pas d'ambigurté), si, $\forall$ a et b $\in \mathrm{A}$, les relations $a x \# b x, a x \neq 0, b x \neq 0$ impliquent $a$ \#b. L'annérde A est dit régulier si tous ses éléments non nuls le sont; dans ce cas, on dit que l'anneau $\overline{\mathrm{A}}$ est régulier.

Pour un élément non nul $x$ de $A$, supposons qu'il existe un entier positif $i$ tel que, $\forall n \geq i$, les relations a $x^{n} \not b x^{n}$, a $x^{n} \neq 0$, $b x^{n} \neq 0$ impliquent $a x^{i} \not b x^{i}$. Notons $i(x)$ le plus petit entier $i$ vérifiant cette condition. $x$ est dit quasi-régulier (ou quasi-régulier fort) d'exposant $i(x)$. Lorsque tous les éléments de A sont quasi-réguliers forts, il est dit quasi-régulier, ou annérde fort. L'annérde sera dit uniformément fort, ou uniformément quasi-régulier, si l'ensemble des exposants $i(x)$ est majoré. On appelle anneau fort l'anneau associé à un annérde fort. .

Pour $x$ non nul de $A$, supposons que, $\forall$ a $\in A$, il existe un entier positif $i(x, a)$ tel que $\forall b \in A, \forall n \geq i$, les relations $a x^{n} \not b x^{n}$, a $x^{n} \neq 0, b x^{n} \neq 0$ impliquent $a x^{i} \# b x^{i}$. Alors, $x$ est dit quasi-régulier strict. Si tous les éléments de A le sont, A est dit para-régulier, ou annéIde strict; $I^{\prime}$ anneau $\overline{\mathbf{A}}$ associé est dit strict.

Pour $x$ non nul de $A$, supposons que, $\forall$ a et $b \in A$, il existe un entier positif $i(x, a, b)$ tel que $\forall n \geqslant i$, les relations $a x^{n} \not b x^{n}$, 
$a x^{n} \neq 0, b x^{n} \neq 0$ impliquent $a x^{i} \# b x^{i}$. Alors, $x$ est dit quasi-régulier large.

Un annétde $A$ unitaire et sans diviseur de zéro est dit un quasidomaine. Il est clair que l'ensemble des éléments réguliers (resp. biréguliers, resp. quasi-réguliers forts) de A est multiplicativement stable.

Si A est sans diviseur de 0 , les notions d'élément de torsion, de moduloide de torsion, se définissent de même que dans le cas des modules. Et l'ensemble des éléments de torsion d'un modulorde est un sous-modulorde.

Soit $u: B \rightarrow B^{\prime}$ un quasi-morphisme d'annérdes (resp. de A-modulordes). Si $\left\{b_{1}, \ldots, b_{n}\right\}$ est une famille d'éléments de $B$, addibles deux à deux, alors les $u\left(b_{i}\right)$ sont addibles et $u\left(\sum_{i=1}^{n} b_{i}\right)=\sum_{i=1}^{n} u\left(b_{i}\right)$. Inversement, si $u$ est un homomorphisme, et si les $u\left(b_{i}\right)$ sont non nuls, et addibles deux à deux, alors on a la même formule. En effet, il en résulte l'addibilité des $b_{i}$, puisque $u$ est un homomorphisme. On dit alors que u se met en facteur. Si certaines images $u\left(b_{i}\right)$ sont nulles, il suffit de les supprimer pour mettre ensuite $u$ en facteur. Par exemple, si $c$ non nul est un élément régulier de l'annérde $A, I$ 'homothétie de $A$ de rapport $c$ est un homomorphisme, et $c$ se met en facteur dans toute somme $\sum_{i=1}^{n} c b_{i}\left(l e s b_{i} \in A\right)$ où les produits $c b_{i}$ sont non nuls et addibles deux à deux.

Soit $u: B \rightarrow B^{\prime}$ un homomorphisme (d'annérdes ou bien de A-modulordes), et soit $K=u^{-1}(0)$ son noyau. La relation $u(x)=u(y)$ implique ou bien $x$ \#y et $x-y \in K$, ou bien $x$ et $y \in K$.

Soient $\mathrm{A}$ un annérde, $\overline{\mathrm{A}} \mathrm{I}$ 'anneau associé, $\mathrm{M}$ un A-modulorde (par exemple A lui-même), $x$ un élément de $M$, et $\gamma=c_{1}+c_{2}+\ldots .+c_{n} \in \bar{A}$ (avec les $c_{i}$ tous non addibles, lorsque $n \geqslant 2$ ). L'élément $\gamma \times$ de $\bar{M}$ n'appartient pas à $M$, sauf dans les deux cas suivants :

1) Les $c_{i} x$ sont deux à deux addibles. Alors, on dit que $x$ agglutine les $c_{i}$; que $\gamma$ est un agglutiné par $x$, ou un coefficient pour $x$; que $\gamma x$ existe, ou a un sens; que les $c_{i}$ sont des co-facteurs pour $x$.

En particulier, soient $a$ et $b$ deux éléments de $A$, avec $a \neq 0$. $a$ est dit un pseudo-diviseur de b, s'il existe un agglutiné $\gamma$ par a tel que $\gamma a=b$. La somme formelle $\gamma$. est dite co-pseudo-diviseur. Si de plus a $n^{\prime}$ est pas diviseur de $b$, ill est dit pseudo-diviseur vrai ou propre de b. Lorsque $b=1$, a est dit pseudo-inversible, et la somme formelle $\gamma$ est appelée 
co-pseudo-inverse. Un annérde sans diviseur ni pseudo-diviseur de o sera dit intègre (c'est alors, en particulier, un intégroíde) ; si de plus il a un élément unité, il sera dit domaine intègre, ou domaine.

2) Dans la décomposition de $\bar{M}$ en somme directe des groupes d'addibilité, $\gamma \mathrm{x}$ admet au plus une composante non nulle $y$, que l'on donnera pour valeur à $\gamma$ x. C'est-à-dire que $\gamma$ s'écrit

$\gamma=\left(c_{1}+\ldots+c_{r}\right)+\left(d_{1}+\ldots+d_{s}\right)+\ldots .+\left(g_{1}+\ldots+g_{v}\right)$ de manière que, dans $M$ :

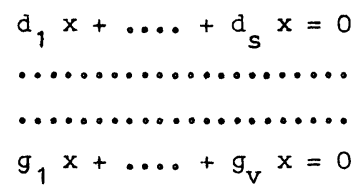

et $y=c_{1} x+\ldots+c_{r} x$ nul ou non. On dira que $\gamma$ est agglutiné large par $x$, que $\gamma$ est un coefficient large pour $x$, que les $c_{i}, d_{j}, \ldots$ sont des co-facteurs larges pour $x$, que $\gamma$ x existe au sens large.

Remarquons que si $c_{1}, \ldots, c_{n}$ sont agglutinés par $x$, ils sont aussi agglutinés par tout multiple a $\mathrm{x}$ de $\mathrm{x}$ (avec $\mathrm{a} \in \mathrm{A}$ ); et aussi, $\forall \mathrm{a} \in \mathrm{A}$, les produits a $c_{1}, \ldots$, a $c_{n}$ sont agglutinés par $x$.

Plus généralement, soient $A$ et $B$ deux annérdes (resp. M et $P$ deux $A$-moduloides) et $u: A \rightarrow B$ (resp. $M \rightarrow P$ ) un quasi-morphisme. Soit $\gamma=c_{1}+\ldots+c_{n}$ (avec les $c_{i}$ tous non addibles, lorsque $n \geqslant 2$ ) un élément te $\bar{A}$ (resp. de $\bar{M})$. En général, la somme formelle $u(\gamma)=\sum_{i=1}^{n} u\left(c_{i}\right)$ n'est pas élément de B (resp. de P), sauf dans les deux cas suivants :

1) les éléments $u\left(c_{i}\right)$ sont deux à deux addibles. Alors, on dit que $u$ agglutine les $c_{i}$, et que $\gamma$ est agglutiné par $u_{\text {. }}$ Quel que soit a $\in A_{\text {. }}$ remarquons que $u$ agglutine aussi les a $c_{i}$;

2) dans la décomposition de $\overline{\mathrm{B}}$ (resp. $\overline{\mathrm{P}}$ ) en somme directe des groupes d'addibilité, $u(\gamma)$ admet au plus une composante non nulle y, qu'on lui donnera pour valeur. Alors, on dit que $\gamma$ est agglutiné large par $u$.

Soit $M$ un $A$-moduloide, et fixons deux éléments $a \in A$ et $x \in M$. La multiplication par $x$ (ou par a) des éléments de A agglutine dans A, et la multiplication par a des éléments de $M$ agglutine dans $M$. Ce sont deux cas particuliers de I'agglutination par un quasi-morphisme de A-modulordes. Dans un cas, le quasi-morphisme $u: A \rightarrow M$ est défini par $u(b)=b x$; dans l'autre cas, $u: M \rightarrow M$ est défini par $u(y)=a y$.

Le phénomène d'agglutination signifie que, dans un anneau gradué $\overline{\mathbf{A}}$, 
ou dans un $\bar{A}$-module gradue $\bar{M}$, des éléments homogènes de grades différents peuvent, après application d'un homomorphisme (de A-modules, ou bien d'anneaux pour le seul $\overrightarrow{\mathbf{A}}$ ) non homogène, donner des images de même grade ; c'est-à-dire que $1^{\prime}$ image d'un élément non homogène peut être homogène. Un cas particulier important est le produit par un élément homogène, soit $x$; on dira que $x$ est régulier si et seulement si, pour tout élément non homogène, la multiplication par $x$ ne le rend pas homogène, sauf trivialement (c'est-à-dire avec des produits nuls, sauf un au plus). Si a et b sont homogènes, et si a divise b, on a appelé a diviseur ou pseudo-diviseur suivant que le co-diviseur était homogène ou non.

D'autre part, l'axiome relatif à l'addibilité pour un quasimorphisme, montre que l'agglutination est une question de grades. En effet, pour les éléments d'un même grade, l'agglutination non triviale a lieu de la même manière ; on peut dire qu'un homomorphisme non homogène agglutine les grades. En particulier, l'agglutination non triviale par $x$ des éléments $c_{i}$ signifie que $\omega\left(c_{i} x\right)=\omega\left(c_{i}\right) \omega(x)$ a la même valeur, pour tout $i$. On dira dans ce cas que $\omega(x)$ agglutine les $\omega\left(c_{i}\right)$.

Pour un quasi-morphisme d'annérdes (resp. de A-modulordes) donné,

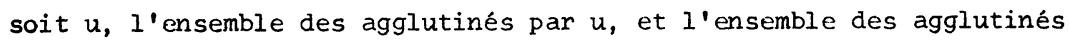
larges par $u, n^{\prime}$ ont pas en général une structure d'annéide (resp. de A-modulolde), mais une structure très voisine : essentiellement, deux quelconques groupes d'addibilité distincts ont en commun un même sous-groupe, mais qui n'est pas forcément réduit à $\{0\}$. D'autre part, I'étude des A-modulordes monogènes conduit à envisager les parties de $\bar{A}$, intermédiaires entre $A$ et $\bar{A}$, et $n^{\prime}$ en différant, outre le support, que par une modification de l'addibilité; ces intermédiaires sont des ensembles d'agglutinés au sens précédent. Nous allons donc, pour la commodité et la clarté de l'exposé, introduire aussi brievement que possible ces structures nouvelles.

\section{$\$ 2$ - ANELS ET MONELS.}

\section{a) Anels - Monels - Graduations :}

On appellera anel tout ensemble non vide $\mathrm{A}$ muni de deux lois intermes : une addition partielle et une multiplication, vérifiant les axiomes:

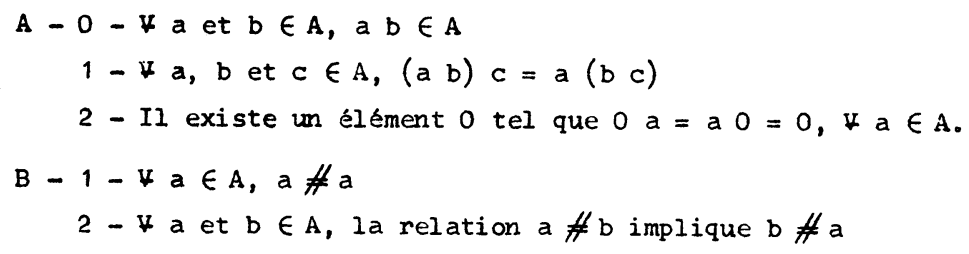


3 - Il existe une partie $\Omega$ de $A$, contenant 0 , et telle que

$$
\forall a \in A, \forall e \in \Omega, \quad a \not \# e
$$

$4-\forall a, b$ et $c \in A$, les relations $a \# b, b \# c, b \notin \Omega$ impliquent a \#c.

Pour a $\notin \Omega$, soit $A(a)=\{x \in A ; x \not \# a\}$ le domaine d'addibilité

de $\mathrm{a} ;$ il est clair que $\mathrm{a} \not \mathrm{b}$ si et seulement s'ils appartiennent à un même domaine. Donc deux tels domaines, ou sont confondus, ou ont $\Omega$ pour intersection. on pose :

$$
D^{*}=\{A(a) ; a \in A, a \notin \Omega\} \quad \text { et } D=D^{*} \cup\{\Omega\} \text {. }
$$

$C$ - Addition : Chaque élément de $D$ est un groupe additif abélien. En particulier, $\forall a$ et $b \in A, a \# b$ implique $a \# a+b$. On voit aisément que 0 est élément neutre de chacun de ces groupes.

D - Relations entre addition et multiplication :

La double distributivité du produit par rapport à l'addibilité, et par rapport à l'addition est vérifiée, et, de plus :

$5-\forall a \in A, \forall$ e $\in \Omega, a$ e $\in \Omega$ et e $a \in \Omega$.

La partie $\Omega$ est appelée le milieu de A. De plus :

l'anel est dit commutatif s'il vérifie :

A $3-\forall a$ et $b \in A, a b=b a$.

l'anel est dit unitaire s'il vérifie:

A 4 - Il existe un élément $1 \in \mathrm{A}$ tel que $1 \mathrm{a}=\mathrm{a} 1=\mathrm{a}, \forall \mathrm{a} \in \mathrm{A}$.

Soit $\mathrm{A}$ un anel. On appellera A-monel à gauche, ou monel à gauche sur $A$, tout ensemble $M$ non vide muni d'une addition partielle, et d'une loi externe partout définie, ayant A pour domaine d'opérateurs, notée multiplicativement, vérifiant les axiomes :

$A-0-\forall a \in A, \forall x \in M, a x \in M$

1 - $\forall$ a et $b \in A, \forall x \in M,(a b) x=a(b x)$

2 - Il existe un élément $0^{\prime}$ de $M$ tel que, $\forall a \in A, \forall x \in M$, a $O^{\prime}=0 x=0^{\prime}$ (on le notera désormais 0 ).

$B-1-\forall x \in M, x \not \#$

$2-\forall x$ et $y \in M$, la relation $x \# y$ implique $y \# x$

3 - Il existe une partie $O$ de $M$, contenant 0 , et telle que : $\forall x \in M, \forall e \in O, x \not e$

$4-\forall x, y$ et $z \in M$, les relations $x \not y, y \not z, y \notin O$ impliquent $x \not z$. Pour $x \notin \mathcal{O}$, soit $M(x)=\{y \in A ; y \not \#\}$ le domaine d'addibilité

de $x$. De même, deux tels domaines ou sont confondus, ou ont $\mathcal{O}$ pour intersection. On pose : $C^{*}=\{M(x) ; x \in M, x \notin O\}$ et $C=C^{*} \cup\{\mathcal{Q}\}$. $C$ - Addition : Chaque élément de $C$ est un groupe additif abélien. En particulier, $\forall x$ et $y \in M, x \# y$ implique $x \# x+y$. Enfin, 0 est élément neutre de chacun de ces groupes. 
D - Relations entre addition et multiplication :

La double distributivité du produit pour l'addibilité et l'addition dans $A$ et $M$ est vérifiée, et de plus :

$$
5-\forall a \in A, \forall x \in \mathcal{O}, a x \in \mathcal{O} ; \forall a \in \Omega, \forall x \in M \text {, a } x \in
$$

La partie $O$ est appelée le milieu de $M$.

Les notions de monel à droite sur $\mathrm{A}$, et de monel unitaire sont

les analogues du cas des modules. Noter que $\Omega$ est un anneau, et $O$ un $\Omega$-module. L'anel est un corpoIde lorsque $A^{*}=A . .\{0\}$ est un groupe multiplicatif (III, $\S 3$ ). Lemme $1: j^{\sharp}$ e $\in \mathcal{O}$ (resp. $\left.\Omega\right), \forall x$ et $y \in M$ (resp. A), la relation $x$ \#y équivaut à $x \# \mathrm{e}+\mathrm{y}$.

C'est évident si $x$ (ou y) est dans $G$. Si $x \notin G$ et y $\notin 0, x \not y$ implique $y \in M(x)$ qui contient $\mathcal{O}$. Donc $e+y \in M(x)$. Propriété fondamentale : $\forall x$ et $y \in M$ (resp. A) et $\forall$ a et $b \in A$, les relations $\mathrm{a} \# \mathrm{~b}$ et $\mathrm{x} \# \mathrm{y}$ impliquent $\mathrm{a} \times \# \mathrm{~b} \mathrm{y}$.

En effet, de mêne que dans le cas des moduloides et des annérdes, la transitivité le montre si a $y \notin 0$, ou si b $x \notin 0$. D'autre part, si $a y$ et $b x \in \mathcal{O}$, de $(a+b) x \not(a+b)$ y on déduit $a x+b x \# a y+b y$ d'ou $a \times \# b$ y grâce au lemme.

Dans la suite, nous supposerons toujours les anels commutatifs.

Par ailleurs, il faut remarquer que bien des résultats suivants concernant les anels et les monels sont encore vrais sans les axiomes $D_{5}$; cependant, on ne considérera pas les objets plus généraux, pour ne pas s'éloigner trop de l'étude qui nous intéresse.

Notons $\bar{A}$ (resp. $\overline{\mathrm{M}}$ ) $I^{\prime}$ 'ensemble des sommes formelles finies d'éléments (non addibles) de A (resp. de $M$ ). L'addition par juxtaposition et réduction des termes addibles en fait évidemment un groupe additif abélien. organisons $\bar{A}$ (resp. $\bar{M}$ ) en anneau (resp. $\bar{A}$-module) en étendant la loi multiplicative de $A$ (resp. de $M$ ) aux sommes formelles par distributivité et réduction des termes addibles. Il est clair alors que $\bar{A}$ est un anneau (resp. $\bar{M}$ un $\bar{A}$-module). $\bar{A} s^{\prime}$ appelle l'anneau associé à l'anel $A$, et $\bar{M}$ le module associé au monel M. Pour $x \in \bar{\Lambda}$, ses composantes dans les groupes d'addibilité sont déterminées modulo le milieu $\Omega$; en quelque sorte, le groupe additif de $\bar{A}$ est la 'somme directe modulo $\Omega$ " de ces groupes, ou "somme quasi-directe". On a la même remarque pour $\bar{M}$. Remarquons qu'un anel de milieu (0) est un annérde, et qu'un monel de milieu (0) sur un annérde, est un moduloide.

Soient $A$ un anel (commutatif), $M$ un $A$-monel, $\Omega$ et $O$ leurs milieux respectifs, et $D$ et $C$ respectivement les ensembles de parties de $A$ et $M$ définis plus haut. On se servira ici d'un abus d'écriture analogue à celui adopté pour les annêrdes et les modulordes : on gardera l'écriture $A(a)$ et $\Omega$ 
(resp. $M(x)$ et $(O)$ pour les opérations sur ces objets en tant que sousensembles de A (resp. $\mathrm{W}$ ) ; on notera $\omega(\mathrm{a})$ et $\omega(0)$ (resp. $\omega(\mathrm{x})$ et $\omega(0)$ ) les mêmes objets, considérés comne éléments de D (resp. de C) pour les opérations qui seront définies dans $D$ (resp. C). D est muni canoniquement d'une loi multiplicative partielle (L), déduite de celle de $A: \forall$ a et $b \in A$ A.. $\Omega$, si $\mathrm{A}(\mathrm{a}) \mathrm{A}(\mathrm{b}) \not \mathcal{\Omega}$, la propriété fondamentale montre l'existence de $\mathrm{c} \in \mathrm{A} . . \Omega$ tel que $A(a) A(b) \subset A(c)$ et l'on pose $\omega(a) \omega(b)=\omega(c)$. L'axiome $D_{5}$ montre que $G \Omega \Omega, \forall G \in D$; on pose donc $\omega(a) \omega(0)=\omega(0)$ et $\omega(0)^{2}=\omega(0)$. De même, l'ensemble $C$ est muni canoniquement d'une loi externe (L) partielle, ayant $D$ comme ensemble d'opérateurs : $\forall a \in A . . \Omega$ et $\forall x \in M . . \mathcal{O}$, si $\mathrm{A}(\mathrm{a}) \mathrm{M}(\mathbf{x}) \not \mathcal{O}$, la propriété fondanentale montre l'existence de $\mathrm{y} \in \mathbf{M} . . \mathcal{O}$ tel que $A(a) M(x) \subset M(y)$ et l'on pose $\omega(a) \omega(x)=\omega(y)$. L'axiome $D_{5}$ montre que, $\forall G \in D, \forall H \in C$, on $a \Omega H \subset O$ et $G O \subset \mathcal{O}$; on pose respectivement $\omega(0) \omega(x)=\omega(0), \omega(a) \omega(0)=\omega(0)$ et $\omega(0) \omega(0)=\omega(0)$. Pour cette loi (L) dans $D, I$ 'élément $\omega(0)$ est absorbant, et pourra être noté 0 ; il en est de même pour l'élément $\omega(0)$ de C. Ceci posé, les définitions d'une graduation $\Delta$ d'un anel $A$, et de sa graduation propre $\Delta \circ$ de support $D$, et les définitions d'une $\Delta$-graduation $\Gamma d^{\prime} u n \Lambda$-monel $M$, et de sa graduation propre $\Gamma_{0}$ de support $C$, sont identiques en tout point à celles relatives aux annérdes et moduloides; nous renvoyons donc aux définitions et remarques du paragraphe correspondant. L'anneau $\bar{A}$ associé à un anel $A$ muni d'une graduation $\Delta$, et le $\bar{A}$-module $\bar{M}$ associé à un $\Lambda$-monel muni d'une $\Delta$-graduation $\Gamma$, seront dits quasi-gradués généralisés. Le grade d'un élément homogène $x \in A . . \Omega$ (resp. $x \in M-G$ ) sera note $\omega(x)$.

b) Sous-monels et idéaux - Notation B X - Loi modulaire : Soient $A$ un anel (commutatif) de milieu $\Omega$, et $M$ un $A$-monel de
milieu $G$. Une partie non vide $P$ de $M$ sera dite un sous-A-monel de $M$, si les lois de $M$ induisent sur $P$ une structure de A-monel. Pour cela, il faut et il suffit que, $\forall \lambda \in \Gamma_{0}, P_{\lambda}=P \cap M_{\lambda}$ soit un sous-groupe additif de $M_{\lambda}$ et que, $\forall a \in A$ et $V x \in P$, a $x \in P$. Ceci équivaut a la condition $P-P \subset P$ et $A P \subset P$ ( $P$ - $Q$ signifiant ici l'ensemble des $p-q$, avec $p \in P, q \in Q$ et $p \# q)$. On voit que le milieu de $P$ est $P \cap \mathcal{O}$. Une partie non vide $\Im$ de $A$ sera dite $\underline{\text { idéal de } A}$ si elle est un sous-monel du A-monel A. Une partie non vide $B$ de $A$ sera dite un sous-anel de $A$ si les lois de $A$ induisent sur $B$ une structure d'anel. Pour cela, il faut et il suffit que, $\forall \alpha \in \Delta_{0}, B_{\alpha}=B \cap A_{\alpha}$ soit un sous-groupe additif de $A_{\alpha}$, et que $¥$ a et $b \in B, a b \in B$. Ceci équivaut à la condition $B-B \subset B$ et $B^{2} \subset B$. On voit que le milieu de $B$ est $B \cap \Omega$.

On a des remarques de calcul sur anels et monels analogues a celles des annerdes ou moduloides. L'expression $\left(a_{1}+\ldots+a_{r}\right)+\left(b_{1}+\ldots+b_{s}\right)$ 
suppose que dans chaque parenthèse les termes soient deux à deux addibles, et que les totaux soient addibles. On en déduit ( $O$ désignant le milieu de $I^{\prime}$ anel ou du monel) : d'abord, soient $\sum_{i=1}^{r} a_{i}=a \notin G$ et $\sum_{j=1}^{s} b_{j}=b \notin O$ deux sommes d'éléments addibles ; si a \#b, quels que soient $i$ et $j$, on $a a_{i} \# b_{j}$. Ensuite, après avoir remplacé par leur total les parenthèses dont le total est dans le milieu, on a le droit de "supprimer les parenthèses".

Cependant, la notion de combinaison linéaire se complique. Soient $M$ un $A$-monel, $\Omega$ et $O$ les milieux respectifs de $A$ et $M, B$ et $X$ des parties non vides respectivement de $A$ et de $M$. On appelle combinaison linéaire simple des éléments de $X$, sur $B$, toute somme finie de la forme $\sum_{x \in X}\left(b_{1}^{x} x+\cdots+b_{r}^{x}(x) x\right)=\sum_{x \in X} \beta^{x} x$, avec les $b_{k}^{x} \in B$, avec $b_{k}^{x}$ et $b_{1}^{x}$ non addibles si $k \neq 1$, et avec les $b_{k}^{x} x$ tous addibles entre eux. On appelle combinaison linéaire des éléments de $X$, sur $B$, toute somme finie de combinaisons linéaires simples dont les totaux sont addibles, c'est-à-dire (après un regroupement éventuel) dont les totaux sont dans le milieu $\mathcal{O}$, sauf un au plus. Par suite, on pourra noter la combinaison $\operatorname{par} \sum_{j}\left\{\sum_{x \in X} \beta_{j}^{x} x\right\}$, (ou bien $\sum_{j}\left\{\sum_{i} \beta_{j}^{i} x_{i}\right\}$ ), où chaque accolade est une combinaison simple, et où des termes $b_{j, k}^{x} x$ et $b_{j, k}^{y} y$ d'accolades différentes sont non addibles lorsqu'aucun n'est dans $\mathcal{O}$. On pourra isoler 1 'accolade dont le total n'est pas dans $\mathcal{O}$. L'ensemble de ces combinaisons linéaires sera noté $B X$, et appelé le produit de $B$ et $X$. En particulier, si B est un idéal ou si $X$ est un sous-monel, $B X$ est évidemment un sous-monel. Si $X=\{x\}$, on pourra noter $B\{x\}=B x$.

Il est clair que l'intersection d'une famille de sous-monels est un sous-monel. Cela justifie la définition suivante : on appelle sous-inonel engendré par une partie $X$ de $M$, et on note $(X)$, le plus petit sous-monel de $M$ contenant $X$. On voit aisément (lorsque $X \neq \varnothing$ ) que $(X)=A X$ lorsque $A$ est unitaire et $(X)=A X+\mathbb{Z X}$ (sens évident : des termes $n_{X} x$ intervenant dans les accolades des combinaisons) lorsque A n'est pas unitaire. Un monel est dit de type fini, s'il possède un système fini $x=\left\{x_{1}, \ldots, x_{n}\right\}$ de générateurs, et il est dit monogène ou cyclique s'il est engendré par un seul élément : $\mathrm{X}=\{\mathrm{x}\}$. Dans ce dernier cas, il pourra être noté $(\mathrm{x})=\mathrm{A} \mathbf{x}+\mathbb{Z} \mathbf{x}$, et il peut être différent de Ax si A n'est pas unitaire. Ces résultats s'appliquent aux idéaux d'un anel. 
Soient $F$ et $G$ deux sous-monels d'un A-monel donné $M$, de graduation propre $\Gamma$. On appelle somme de $F$ et $G$, et on note $F+G$, le sous-monel engendré par F U G. Cette somme est I'ensemble des sommes finies $\sum_{\alpha \in \Gamma^{*}}\left(f_{\alpha}+g_{\alpha}\right)$ ou pour tout $\alpha$, on a $f_{\alpha} \# g_{\alpha}$ et ou, si $\alpha \neq \beta$, on a $\left(f_{\alpha}+g_{\alpha}\right) \not \#\left(f_{\beta}+g_{\beta}\right)$. Après une éventuelle réduction, on constate que les totaux $\left(f_{\alpha}+g_{\alpha}\right)$ sont dans le milieu $\mathcal{O}$ sauf un au plus, et les $f_{\alpha}$ (resp. $g_{\alpha}$ ) ne sont pas dans $\boldsymbol{O}$, sauf un au plus. Donc, tout $x \in F+G$ pourra s'écrire $x=f_{\gamma}+g_{\gamma}+\sum_{\alpha \neq \gamma}\left(f_{\alpha}+g_{\alpha}\right)$ avec les conditions suivantes : lorsque $x \notin \mathcal{O}, f_{\gamma}$ et $g_{\gamma}$ sont addibles a $x_{0}$ les $f_{\alpha}$ et $g_{\alpha}$ (qui figurent) ne sont pas addibles $a x$, et chaque total $\left(f_{\alpha}+g_{\alpha}\right)$ est dans $\mathcal{O}$; Iorsque $x \in \mathcal{O}$, ou bien $x=\sum_{\alpha}\left(f_{\alpha}+g_{\alpha}\right)$ avec $f_{\alpha}$ et $g_{\alpha}$ addibles, et non situés dans $\mathcal{O}$, et $\left(f_{\alpha}+g_{\alpha}\right) \in \mathcal{O}$, ou bien $x=f_{\gamma}+g_{\gamma}$ avec $f_{\gamma}$ et $g_{\gamma}$ dans $(0$.

Théorème 1 : (loi modulaire dans les monels)

Soient E, F, G trois sous-monels d'un A-monel donné M de milieu $O$. Si $E \supset F$, et si F (resp. G) est comparable à $O$ (c'est-à-dire $F \subset G$ ou $F \supset G$ ) on $a \cap(F+G)=F+(E \cap G)$.

En résumé, la loi modulaire classique, encore vraie pour les modulordes, n'est pas forcément vérifiée dans les monels, comme le montre l'exemple qui suit.

L'inclusion $E \cap(F+G) \supset F+(E \cap G)$ est évidente. Soit $x \in F+G$. Dans l'écriture précédente de $x:$ ou bien $F \subset \mathcal{O}$, donc les $f_{\alpha}$ et $g_{\alpha}$ sont nuls, et $\mathrm{x}=\mathrm{f}_{\gamma}+\mathrm{g}_{\gamma}$. Ou bien $\mathrm{F} \supset \mathcal{O}$, et la somme $s=\sum_{\alpha \neq \gamma}\left(f_{\alpha}+g_{\alpha}\right)$ est dans $\boldsymbol{O}$, donc dans $F$, donc $f_{1}=f_{\gamma}+s \in F$ et $x=f_{1}+g_{\gamma}$. Par suite, si $x \in E \cap(F+G)$, il s'écrit $x=f+g$ avec $f \in F \subset E, g \in G, f+g \in E$. On a $g=x-f \in E$, donc $g \in E \cap G$ et $x \in F+(E \cap G)$.

Corollaire : Soient E, F, G trois sous-monels d'un A-monel M de milieu $G$, tels que E 2 F. Si F (resp. G) est comparable à $G$, et si $E \cap G=F \cap G$ et $E+G=F+G$, on $a E=F$. En particulier, les relations $\mathrm{E} \supset \mathrm{F}, \mathrm{E} \cap \mathcal{O}=\mathrm{F} \cap \mathcal{O}, \mathrm{E}+\mathcal{O}=\mathrm{F}+\mathcal{O}$ impliquent $\mathrm{E}=\mathrm{F}$.

En effet $E=E \cap(E+G)=E \cap(F+G)=F+E \cap G=F+(F \cap G)=F$. Noter que théorème et corollaire s'appliquent aux idéaux d'un anel.

Exemple contraire ou $F+G$ est différent de $I$ 'ensemble $S$ des sommes 
$f+g($ avec $f \in F, g \in G)$, et où la loi modulaire classique est en défaut. Soit L le $\mathbb{Z}$-module libre $L=\mathbb{Z}$ a $+\mathbb{Z}$ b $+\mathbb{Z} c+\mathbb{Z}$ d, de base $\{a, b, c, d\}$. Soient $M_{1}$ et $M_{2}$ des $\mathbb{Z}$-modules isomorphes à $L^{2}$ (nous les noterons comne $L^{2}$, par abus d'écriture). Soit $M$ le $\mathbb{Z}$-monel fórmé de la réunion de $M_{1}$ et $M_{2}$, ces derniers ayant, par définition, en . commun le milieu $\mathcal{O}=\mathbb{Z}(a, a)+\mathbb{Z}(b, b)$.

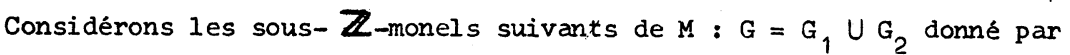
$G_{1}=\mathbb{Z}(c, 0) \subset M_{1}$ et $G_{2}=\mathbb{Z}(d, 0) \subset M_{2}$. Puis $F$ donné par $F_{1}=\mathbb{Z}(a+c, a)$ et $F_{2}=(b+d, b)$. Puis $H$ donné par $H=\mathbb{Z}(a+b$, $a+b) \subset \mathcal{O}$ et $E=F+H$. $D^{\prime}$ abord $F \cap \mathcal{O}=(0,0)$. Ensuite, $E_{1}$ est formé des couples $(n(a+c)+p(a+b): n a+p(a+b))$ avec $n, p \in \mathbb{Z}$, et de même $E_{2}$ des couples. $(n(b+d)+p(a+b), n b+p(a+b))$. Soit $y \in E \cap G ;$ si par exemple $y \in \mathbb{M}_{1}$, sa deuxième composante $n a+p(a+b)$ doit être nulle, donc on a $\mathrm{n}=\mathrm{P}=0$, et $\mathrm{E} \cap \mathrm{G}=(0,0)$.

Il est clair que $E \cap(F+G)$ contient $F+(E \cap G)=F$. Prenons $f_{1}=(a+c, a) \in F_{1}, g_{1}=(-c, 0) \in G_{1}, f_{2}=(b+d, b) \in F_{2}, g_{2}=(-d, 0) \in G_{2}$. Alors $f_{1}+g_{1}=(a, a)$ est dans $G$, non dans $E$, et il en est de même pour $f_{2}+g_{2}=(b, b)$. Leur soinme $x=\left(f_{1}+g_{1}\right)+\left(f_{2}+g_{2}\right)=(a+b, a+b)$ est élément de $\mathrm{H}$ et de $\mathrm{F}+\mathrm{G}$, donc de $\mathrm{E}=\mathrm{F}+\mathrm{H}$ et de $\mathrm{E} \cap(\mathrm{F}+\mathrm{G})$. Noter que $\mathbf{x}$ ne peut s'écrire $f+g$ (avec $f \in F, g \in G$ ), donc que $S$ et $F+G$ sont différents. D'autre part, il est clair que $x=(a+b, a+b) \notin F$. Par suite, on a $E \cap(F+G) \neq F=F+(E \cap G)$.

c) Anels et monels quotients :

Congruence modulo un sous-monel : $P$ étant un sous-A-monel de $M$, soit $R$ la relation d'équivalence dans $M$ la plus fine telle que les relations $x, y \in M, x \# y, x-y \in P$ impliquent $x R$. Nous noterons dans la suite $x+P$ (ou $x \in M$ est donné) l'ensemble des éléments $x+p$, avec $p \in P, p \# x$. Deux éléments $x$ et $y$ de $M$ seront dits congrus modulo $P$, et on notera $x \equiv y(P)$ (ou bien $x \equiv y s^{\prime} i l$ n'y a pas d'ambigurté) s'ils vérifient l'une quelconque des conditions équivalentes suivantes:

I $-x R y$

II - ou bien $x \not$ $y$ et $x-y \in P$, ou bien $x$ et $y$ ne sont pas addibles, mais il existe $e \in G$ tel que $x-e \in P$ et $y-e \in P$

III - ou bien $x \# y$ et $y \in x+P$, ou bien $x$ et $y$ ne sont pas addibles, mais il existe $e \in \mathcal{O}$ tel que $x$ et $y \in e+D$

IV - il existe $z \in M$ tel que $x$ et $y \in z+P$.

II et III sont deux manières de dire la même condition, et elles 
entrainent évidemment IV. Inversement, si IV est vérifiée, et si $z \notin \mathcal{O}$, soient $x=z+p$ et $y=z+p^{\prime}$; par transitivité par $z$, les éléments $z$, $p$ et $p^{\prime}$ sont addibles, donc aussi $x$ et $y$; on a donc $x-y=(z+p)-\left(z+p^{\prime}\right)=p-p^{\prime} \in P$. La restriction de $R$ à un groupe d'addibilité ${ }_{\lambda}$ est évidemment la congruence modulo $P_{\lambda}=P \cap M_{\lambda}$. Si une classe $\xi$ modulo $R$ rencontre $O$, soit e $\in \circlearrowleft \cap \xi$, puisque $\xi_{\lambda}=\xi \cap M_{\lambda}$ égale e $+P_{\lambda}$, la transitivité de $R$ par e implique $\xi=\bigcup_{\lambda \in \Gamma_{0}} \xi_{\lambda}=e+P$. Si au contraire $\xi$ ne rencontre pas $\mathcal{O}, \forall x \in \xi$, on a $x \notin \mathcal{O}$ et $\xi \supset x+P$. Alors, si $x$ et $y$ sont deux éléments non addibles tels que $x+P$ et $y+P$ ne rencontrent pas $\mathcal{O}$ (donc, d'après ce qui précède, sont contenus dans des classes modulo $R$ ne rencontrent pas $\mathcal{O}$ ), aucun élément de $x+P$ 'est addible à un élément de $y+P$; la dernière condition de la définition de $R$ est donc vide entre élément de $x+P$ et élément de $y+P$; $R$ étant la plus fine possible, $x+P$ et $y+P$ scront donc dans des classes modulo $\mathrm{R}$ distinctes. Donc, si $\xi$ ne rencontre pas $\mathcal{O} \forall x \in \xi$, on a $\xi=x+P$. on notera $M / P$ l'ensemble quotient correspondant, et $\varphi$ la surjection canonique $: M \rightarrow M / P$. Les classes modulo $P$ sont donc, $d$ 'une part les classes $e+P$ pour $e \in \mathcal{O}$, d'autre part les classes $x+P$, pour $x \in M, x \notin e+P, \forall$ e $\in \mathcal{O}$. Les mêmes résultats sont valables en remplaçant $M$, $\mathcal{O}$ et $P$ respectivement par un anel A, son milieu $\Omega$, et un idéal $\Im$. On notera $A / \Im$ l'ensemble quotient correspondant, et $\varphi$ la surjection canonique : A $\rightarrow \mathrm{A} / \mathrm{I}^{\circ}$

Lemme 2 : La congruence modulo $P$ est normale par rapport à l'addibilité, et compatible avec l'addition et avcc le produit par un scalaire, c'est-à-dire :

(1) S'il $n$ 'existe aucun $e \in \mathcal{O}$ tel que $x \equiv e$, la relation $x \equiv y$ implique $x \not y$.

(2) Les relations $x \equiv y, x^{\prime} \equiv y^{\prime}, x \# x^{\prime}$ et $y \# y^{\prime}$ impliquent $x+x^{\prime} \equiv y+y^{\prime}$.

(3) $\forall a \in A, x \equiv y$ implique $a x \equiv a y$.

Réciproquement, dans $M$, toute relation d'équivalence normale et compatible au sens précédent, est une congruence modulo un sous-monel de $M$. (Noter que la première partie de la condition (1) équivaut à $\times \notin \mathbf{O}+\mathrm{P}$ )

En effet, (1) résulte de III. (3) résulte de II, car si $x-y \in P$ (resp. $x-e \in P$ ), comme $P$ est un sous-monel, a $x-a y \in P$ (resp. a $x-a$ e $\in P$, et a $e \in \mathcal{O}$ ). Pour (2), si par exemple

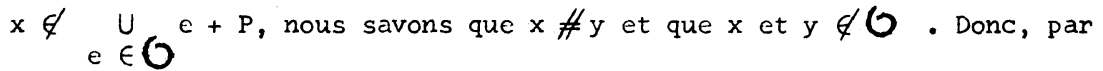
transitivité, les éléments $x, y, x^{\prime}$ et $y^{\prime}$ sont deux à deux addibles : commc alors $x-y$ ct $x^{\prime}-y^{\prime}$ sont dans $P$ (grace à III) on a : $\left(x+x^{\prime}\right)-\left(y+y^{\prime}\right)=(x-y)+\left(x^{\prime}-y^{\prime}\right) \in P$, donc $x+x^{\prime} \equiv y+y^{\prime} \cdot S i$, 
au contraire, tous les éléments $x, y, x^{\prime}$ et $y^{\prime} \in \mathcal{O}+P$, alors (gráce à IV) $J_{\epsilon}$ et $e^{\prime} \in \mathcal{O}$ tels $q u^{\prime}$ on ait $x-e \in P, y-e \in P, x^{\prime}-e^{\prime} \in P$ et $y^{\prime}-e^{\prime} \in P$. Mais on sait que $x \# x^{\prime}$ équivaut a $x-e \# x^{\prime}-e^{\prime} ;$ on a donc $\left(x+x^{\prime}\right)-\left(e+e^{\prime}\right)=(x-e)+\left(x^{\prime}-e^{\prime}\right) \in P$, et de même pour $y+y^{\prime} ; d^{\prime}$ où enfin $x+x^{\prime} \equiv y+y^{\prime}$.

Réciproquement, soit $S$ une relation d'équivalence dans $M$, vérifiant les conditions du lemme. On pose $P=\{x \in M ; x S 0\}$. Que $P$ soit un sousmonel se vérifie trivialement. Comparons $S$ et la congruence modulo $P$. Lorsque $\mathrm{x} \# \mathrm{y}, \mathrm{x} \equiv \mathrm{y}$ équivaut à $(\mathrm{x}-\mathrm{y}) \mathrm{S} 0$ par définition de $\mathrm{P}$; ceci, grace à la condition (2) pour $S$, équivaut à $x \mathrm{~S}$ y (ajouter $\stackrel{+}{\mathrm{y}}$ aux deux membres). Lorsque $x$ et $y$ ne sont pas addibles, $x \equiv y$ signifie (II) qu'il existe $e \in(O$ tel que $x-e \in P$ et $y-e \in P$, et nous venons de voir que cela équivaut à $\mathrm{x} S \mathrm{e}$ et y $\mathrm{S}$ e. D'où $\mathrm{x} \mathrm{S}$. Inversement, $\mathrm{x} S \mathrm{y}$ implique (condition (1) pour $\mathrm{s}$ ) qu'il existe $e \in O$ tel que $x \mathrm{~S} e$, d'où y $s$ e par transitivité de $s$.

on notera $\varphi: M \rightarrow M / P$ la surjection canonique de $M$ sur l'ensemble quotient $\mathrm{H} / \mathrm{P}$ (qui pourra aussi etre noté $M-P$ ).

Dans un anel $\mathrm{A}$, on a les mêmes résultats pour les classes et la congruence modulo un idéal $\mathfrak{J}$ (notonsła encore $a \equiv b(\mathfrak{I})$, ou $a \equiv b$ ), sauf qu'ici cette congruence est considéréc dans A en tant qu'anel des opérateurs, et pas seulement dans A en tant que A-monel. A cause de cela, on a non seulement : $\forall \mathrm{a}, \mathrm{b} \equiv \mathrm{b}^{\prime}$ implique $\mathrm{a} \mathrm{b} \equiv \mathrm{a} \mathrm{b}^{\prime}$, mais la condition (qui aurait été plus forte dans le cas non commutatif) :

( $\left.3^{\prime}\right)$ Les relations $a \equiv a^{\prime}$ ct $b \equiv b^{\prime}$ impliquent $a b \equiv a^{\prime} b^{\prime}$.

Montrons maintenant que $\mathrm{M} / \mathrm{P}$ est un $\mathrm{A}-$ monel et que $\mathrm{A} / \mathrm{g}$ est un anel. Cette rédaction, non détaillée, sera faite pour les A-monels. Pour les anels, les modifications sont minimes.

Soient donc $P$ un sous-A-monel de $M$, et $\varphi$ la surjection canorique : $M \rightarrow M / P$. Organisons $\mathrm{H} / \mathrm{P}$ en A-monel ainsj : $\forall$ a $\in A, \forall \varphi(x) \in M / P$, on pose a $\varphi(x)=\varphi(a x)$, définition indépendante (compatibilité (3)) du choix de $x$ dans la classe. Les axiomes (A) se vérifient trivialement. Deux classes $\xi$ et $\xi^{\prime} \in M / P$ seront dites addibles si et seulement si elles contiennent un élément addible (soient $x$ et $x^{\prime}$ respectivement); alors, on pose $\xi+\xi^{\prime}=\varphi\left(x+x^{\prime}\right)$. La définition est indépendante (compatibilité (2)) du choix du couple $\left(x, x^{\prime}\right)$. La classe somme $\varphi\left(x+x^{\prime}\right)=\left(x+x^{\prime}\right)+P$ contient $I^{\prime}$ ensemble des $x+x^{\prime}+p$ (où $p \in P, p$ addible à $x$ et $x^{\prime}$ ), mais peut en être différente (prendre $\left.x^{\prime}=-x \notin P\right)$. M/P satisfait aux axiomes des A-monels ; la vérification, longue, est sans difficulté. Faisons deux remarques : on prend pour milieu $\mathcal{O}_{1}$ de $\mathrm{H} / \mathrm{p}$ I'ensemble des $\varphi(\mathrm{e})$ distincts, pour e $\in \mathcal{O}$. Tout groupe d'addibilité de $\mathrm{M} / \mathrm{P}$ est isomorphe au groupe additif quotient d'un 
groupe $M_{\lambda}$ par le sous-groupe $P \cap M_{\lambda}$ (restreindre à $M_{\lambda}$ la partition de $M$ par la congruence modulo $P$ ).

Dans le cas du quotient $A / \Im$, notons encore $\varphi$ la surjection canonique $A \rightarrow A / \Im$. Pour $\varphi(a)$ et $\varphi(b) \in A / \Im$, on posera $\varphi(a) \varphi(b)=\varphi(a b)$, définition indépendante (condition $\left(3^{\prime}\right)$ ) du choix de a et de $b$ dans les classes correspondantes.

d) Homomorphismes - Régularité :

Soient $M$ et $M_{1}$ deux A-monels, et $\mathcal{O}_{\text {et }} G_{1}$ leurs milieux respectifs. Une application $f: M \rightarrow M_{1}$ est dite homomorphisme de $A$-monels si, $\forall x$ et $y \in M$, $\forall a \in A$ :

1 - la relation $x \not y$ implique $f(x) \# f(y)$, et on a $f(O) \subset O_{1}$

$1^{\prime}$ - les relations $f(x) \# f(y), f(x) \notin O_{1}, f(y) \notin O_{1}$ impliquent $x \not \# y$

2 - la relation $x$ \#y implique $f(x+y)=f(x)+f(y)$

$3-f(a x)=a f(x)$.

Si $f$ vérifie $(1,2,3)$, mais non ( $\left.1^{\prime}\right)$, il est dit homomorphisme

faible ou quasi-morphisme. Remarquer que lorsque l'image $f(M) n^{\prime}$ 'est pas

contenue dans un seul groupe d'addibilité de $M_{1}$, la première partie de la condition (1) implique $f(O) \subset G_{1}$.

Soient $A$ et $A_{1}$ deux anels de milieux respectifs $O$ et $O_{1}$. Les notions analogues d'homomorphisme et quasi-morphisme d'anels se définissent de même, en remplaçant (3) par : 3 bis - $\forall$ a et $b \in A, f(a b)=f(a) f(b)$. Un homomorphisme (resp. quasi-morphisme) d'anels est dit unitaire si $A$ et $A_{1}$ sont unitaires et si $f(1)=1$. Un homomorphisme $f$ (d'anels ou de monels) sera dit un isomorphisme $s^{\prime}$ il est bijectif et si $f(O)=G_{1}$.

Pour les anneaux [resp. modules] qyasi-gradués, un homomorphisme est dit homogène (resp. quasi-homogène) si sa "restriction aux parties homogènes est un homomorphisme (resp. quasi-morphisme) (voir I, $\S 3$ ).

Anels réguliers, forts, stricts. Soit $A$ un anel de milieu $\Omega$. Pour un élément $x$ de $A, x \notin \Omega$, la notion de régularité est analogue à celle donnée dans les annéfdes : $x$ est dit régulier si, quels que soient $a$ et $b$ dans $A$, les relations a $x \not$ b $x, a x \notin \Omega, b \times \notin \Omega$ impliquent $a \# b$. L'anel $A$ est dit régulier si tout élément hors du milieu est régulier. De même, pour définir la quasi-régularité forte ou stricte, on remplacera dans les hypothèses (voir $\S 1$ ) $a x^{n} \neq 0$ et $b x^{n} \neq 0$ par $a x^{n} \notin \Omega$ et $b x^{n} \notin \Omega$. On a de même les notions d'anel fort, d'anel strict.

Soient $A$ un anel de milieu $\Omega$ et de graduation $(\Delta, \theta), \Im$ un idéal de $A$, et $\varphi: A \rightarrow A / \Im$ l'homomorphisme canonique. Posons $D^{*}=\{A(a) ; a \in A . . \Omega\}$ et 
$D=D^{*} U\{\Omega\}$; comme élément de $D, \Omega$ pourra être noté 0 . De même, dans $A / \Im$, notons $D_{1}^{*}$ et $D_{1}=D_{1}^{*} \cup\left\{\Omega_{1}\right\}$. Définissons une application $\psi: D \rightarrow D_{1}$, déduite de $\varphi$ ainsi : on pose $\psi(\Omega)=\{\varphi(\omega) ; \omega \in \Omega\}=\{\omega+\Im ; \omega \in \Omega\}=\Omega_{1}$ et pour tout a $\in$ A.. $\{U(\omega+\Im)\}$, on pose : $\forall(A(a))=(A / \Im)_{a+\Im}=\Omega_{1} \cup\left\{\varphi\left(a^{\prime}\right) ; a^{\prime} \in A_{a} \cdot .\left\{U_{\omega \in \Omega}(\omega+\Im)\right\}\right\}$.

Il est clair que $\psi$ est surjective, et injective sur l'image réciproque $\bar{\psi}^{1}\left(D_{1}^{*}\right)$. Ceci, joint aux propriétés de la multiplication dans $A / \mathfrak{S}$, montre ( $I, \S 3$, définition d'une graduation) que $(\Delta, \theta)$ étant une graduation de $A$, il en résulte que $(\Delta, \downarrow \circ \theta)$ est une graduation de $A / \Im^{\circ}$

De même, si $M$ est un A-monel de $\Delta$-graduation $\Gamma$, et si $P$ est un sous-monel de $M$, $\Gamma$ peut être considéré canoniquement comme une $\Delta$-graduation de $M / P$.

\section{$\S 3$ - STRUCTURE DES AGGLUTINES.}

a) Agglutinés simple et large par un quasi-morphisme

Soient $A$ un anel (commutatif), il et if ${ }_{1}$ deux A-monels, et $\Omega$, $\mathcal{O}$ et $\mathcal{O}_{1}$ leurs milieux respectifs. Soit $f: M \rightarrow M_{1}$ un quasi-morphisme de monels. Un élément $\xi=\sum_{i=1} x_{i}$ (les $x_{i}$ deux à deux non addibles) de $\bar{M}$ sera dit agglutiné par $f$ si, $\forall i$ et $j \leqslant s, f\left(x_{i}\right) \not f\left(x_{j}\right)$; le lemme 1 du paragraphe (2) montre que cette définition est indépendante de l'expression de $\xi$; alors, $\forall$ a $\in A$, a $\xi=\sum_{i=1}^{S} a x_{i}$ est aussi agglutiné par $f . L$ 'ensemble de ces agglutinés est noté

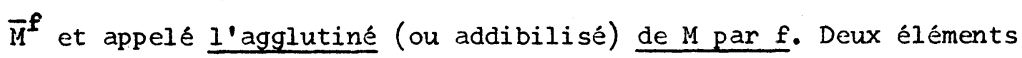
$\xi$ et $\xi^{\prime}=\sum_{j=1}^{r} x_{j}^{\prime}$ de $\bar{M}^{f}$ seront dits addibles (dans l'agglutiné), ou addibles par $f$ si, $\forall i \leqslant s, \forall j \leqslant r, f\left(x_{i}\right) \not f\left(x_{j}^{\prime}\right)$. On note alors $\xi \#_{f} \xi^{\prime}$ (ou sans l'indice $f s^{\prime} i l n^{\prime} y$ a pas d'ambigurté). Leur somme est par définition obtenue par juxtaposition et réduction des termes addibles dans M. Cette multiplication et cette addition partielle font de $\bar{M}^{f}$ un A-monel. D'abord, le milieu de $\bar{M}^{f}$ est $\mathcal{O}^{\prime}=\left\{\xi \in \bar{M}^{f} ; \forall i, f\left(x_{i}\right) \in G_{1}\right\}$. $G^{\prime}$ est le sous-groupe additif de $\bar{M}$ engendré par $f^{-1}\left(O_{1}\right)$. Vérifions los axiomes $B_{3}$ et $B_{4}: \forall \xi \in \mathcal{O}^{\prime}$, 
$\forall \eta=\sum_{j=1}^{r} y_{j} \in \bar{M}^{f}$ il est clair que $f\left(x_{i}\right) \not f\left(y_{j}\right)$, donc que $\xi$ et $\eta$ sont addibles par $f$. Si $\xi$, $\eta$ ct $\zeta \in \mathbb{M}^{-\dot{f}}$ sont addibles par $f$, et si $\eta \notin G^{\prime}$, il existe $y_{1}$ dans la somme $\eta$ tel que $f\left(y_{1}\right) \notin \mathcal{O}_{1}$; donc $f\left(y_{1}\right)$ transmet l'addibilité, $\forall i$ et $\forall k$, entre $f\left(x_{i}\right)$ et $f\left(z_{k}\right)$. D'autre part, la propriété fondamentale entraine $I$ 'axiome $D_{3}$ de $\bar{H}^{f}$, car a \# b et $f\left(x_{i}\right)$ \#f( $\left.x_{j}\right)$ entrainent $f\left(a x_{i}\right)=a f\left(x_{i}\right)$ \#b $f\left(x_{j}\right)=f\left(b x_{j}\right)$. Pour les autres axiomes, la vérification est triviale.

Soient $A$ et $A_{1}$ deux anels (commutatifs) de milieux $\Omega$ et $\Omega_{1}$ respectivement. Soit $f: A \rightarrow A_{1}$ un quasi-morphisme d'anels. L'agglutiné par $f$, soit $\bar{A}^{f}$, cst un anel. La vérification est analogue. Remarquons que l'axiome Ao et la distributivité du produit par rapport à l'addibilité sont conséquences de la propriété fondamentale; pour l'axiome A。 par exemple, $\xi$ et $\eta \in \bar{A}^{f}$ signifie que (avec les notations habituelles), $\forall i$ et $j, f\left(x_{i}\right) \not f f\left(x_{j}\right)$, et $\forall k$ et $1, f\left(y_{k}\right) \# f\left(y_{1}\right)$, d'où $f\left(x_{i} y_{k}\right)=f\left(x_{i}\right) f\left(y_{k}\right) \# f\left(x_{j}\right) f\left(y_{1}\right)=f\left(x_{j} y_{1}\right)$. Si nous considérons ces résultats dans le cas particulier d'un modulớde $M$ sur un annérde $A$ (commutatif), il faut bien remarquer qu'un agglutiné de $M$ par un quasi-morphisme $f$ est un A-monel, et non un A-moduloide, lorsque $\bar{f}^{-1}(0)$ est différent de $\{0\}$. De mêne l'agglutiné d'un annérde sera en général un anel.

Lorsque $M$ est un A-monel, le $\bar{A}$-module $\bar{M}$ peut être considéré comme un $\bar{A}$-monel d'autant de façons qu'il y a de choisir son milieu $\mathcal{O}^{\prime}$. Celui-ci sera un sous $-\bar{A}$-module de $\bar{M}$, contenant le milieu $G$ de $M$. Nous ne distinguons pas les deux structures (moncl et module) lorsque $\mathcal{O}^{\prime}=\{0\}$. Lorsque $G \neq\{0\}$, il y a danger à les confondre, par exemple dès que l'on envisage un

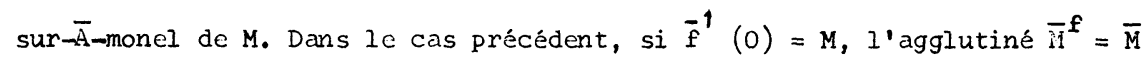
est un monel, sur $A$ et sur $\bar{A}$, égal à son milieu, bien qu'il ait aussi une structure sous-jacente de $\bar{A}$-modulorde (et de $\bar{A}$-module). On a des remarques analogues sur I'anneau $\bar{A}$ d'un anel A.

Soient $\mathrm{A}$ un anel (commutatif), et il un A-monel. Pour un élément fixé a de $A, I$ 'homothétie $h$ de $M$, définie $\operatorname{par} h(x)=a x$, est un quasimorphisme. L'agglutiné de $M$ par $h$ est un A-monel qui sera noté $\bar{M}^{a}$, et appelé l'agglutiné de $\mathrm{I}$ par a.

De même, pour un élément fixé $x$ de il (resp. A), l'application 
$f: A \rightarrow M(r e s p . A \rightarrow A)$ définie par $f(a)=a x$ est un quasi-morphisme de A-monels. $\bar{A}^{f}$ est un A-monel qui sera noté $\bar{A}^{X}$ et appelé l'agglutiné (ou addibilisé) de $\mathrm{A}$ par $\mathrm{x}$. Son addibilité pourra être notée $\#_{\mathbf{x}}$, afin d'éviter les confusions. Pour $x$ non nul dans $A$, si la suite $\operatorname{des} \bar{A}^{x^{n}}, n=1,2,3, \ldots$, est stationnaire, $\mathrm{x}$ est quasi-régulier.

Soient A un anel (commutatif) et is un A-monel, munis de graduations. Soient $\Omega$ et $\mathcal{O}$ leurs milieux. On notera $w(z)$ le grade d'un élément $z$ de $A$, ou de $M$, non situé dans le milieu. Soient $a, b \in A$ et $x \in M$ (resp. A). Par définition d'une gracuation, la condition a $x \notin \mathcal{O}$ (resp. $\Omega$ ) implique $\omega(\mathrm{a} x)=\omega(\mathrm{a}) \omega(\mathrm{x})$. De là il résulte, si a b $\mathrm{x} \not \ell(0$ (resp. $\Omega)$ I'associativité du produit des grades :

$$
[\omega(a) \omega(b)] \omega(x)=w(a)[w(b) \omega(x)] \text {. }
$$

Lemme 1 : Lorsque la graduation de A est commutative, soient des éléments non situés dans le milieu $a, b, c, d \in A$ et $x \in M$ (resp. A) tels que $a x \# b x$ et $c x \not d x$. Si de plus le produit des grades de $b$, $c$ et $x$ (ou bien de $a, d$ et $x$ ) est associatif, on $a$ : a $c x \not b d x$.

Si $a c x$ et $b$ d $x$ nc sont pas dans le milieu, $a x$ et $b x$ non plus, par l'axiome $D_{5^{\prime}}$. Donc, par $I^{\prime}$ hypothèsc du 1 emme $\omega(\mathrm{a} x)=\omega(\mathrm{b} x)$ et, par la remarque précédente :

$\omega(\mathrm{a} c \mathrm{x})=\omega(\mathrm{c}) \omega(\mathrm{a} x)=\omega(\mathrm{c}) \omega(\mathrm{b} x)=\omega(\mathrm{c})[\omega(\mathrm{b}) \omega(\mathrm{x})]$. On montre de meme que $\boldsymbol{\omega}(\mathrm{b} d \mathrm{x})=\boldsymbol{\omega}(\mathrm{b})[\omega(\mathrm{c}) \boldsymbol{\omega}(\mathrm{x})]$. La démonstration s'achève par l'associativité.

Corollaire 1 : Supposons la graduation de l'anel A associative et commutative, et celle du A-monel $\mathbb{H}$ associative. Si $x \in M$ (resp. A) agglutine deux sommes formelles d'éléments de A, il agglutine leur produit. En particulier, $\overrightarrow{\mathrm{A}}^{\mathbf{x}}$ est un anel.

Corollaire 2: En plus des conditions du corollaire 1, supposons que $A$ soit un annérde, que $\mathrm{I}$ soit an $\mathrm{A}$-modulorde, et que $\mathrm{x} \neq 0$ ne soit pas élément de torsion (resp. diviseur de 0 ). Alors, $\overrightarrow{\mathrm{A}}$ est un annérde.

Ces corollaires sont des conséquences immédiates du lemme. Pour le corollaire 2, I'hypothèse sur $x$ montre que le milieu de $\vec{A}^{x}$ est $\{0\}$. L'exemple contraire suivant montre l'utilité de l'associativité, non. seulenent pour le lemme, mais aussi pour le corollaire $c$.

Exemple contraire 1 : Soit $\Delta_{0}$ le monorde commutatif formé de 1 'annulateur 0 et des monômes à quatre variables a, b, C, d avec les relations $\mathrm{a} \cdot \mathrm{d}=\mathrm{b} \mathrm{c}=0$. Soient $\mathrm{k}$ le corps à deux éléments, $\overline{\mathrm{A}} \mathrm{I}$ 'anneau gradue de type $\Delta_{0}$ des polynômes sur $k$ en $a, b, c, d$ (avec $a d=b c=0$ ), et enfin $A$ l'annérde associé (on peut considérer $\Delta_{0}$ comme support de A). $\Delta_{0}$ est la graduation 
propre de $A$, et est commutatif et associatif. Notons H la plus petite partie de $\bar{A}$, contenant $A$, qui soit un A-modulorde où a \# b et $c \# d$. Partant de $A$, on étend donc l'addibilité en imposant ces conditions, et leurs conséquences dues a la distributivité du produit par un élément de $A$, et à la transitivité par les éléments non nuls. Si, pour $n \geq 0$, on a dans ii : $a^{n} \# b^{n}$, le produit par a montre $a^{n+1} \#$ a $b^{n} ;$ de $a \# b$, on déduit grace au produit par $b^{n} \in A$, $a b^{n} \not b^{n+1} ;$ comme $a b^{n} \neq 0$, il vient $a^{n+1} \# b^{n+1}$; donc, $\forall$ l'entier $n \geq 0$,

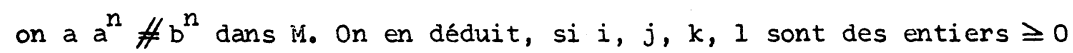
vérifiant $i+j=k+1$ que $a^{i} b^{j} \# a^{k} b^{l}$ dans $M$. Il en est de même pour le couple des variables $c$ et $d$. Finalement, $M$ est formé uniquement, outre les éléments de $A$, des polynomes (sur $k$ ) homogènes soit en a et $b$, soit en $c$ et $d$. Remarquons que le A-modulor̂de i est engendré par 1, considéré comme élément de $M$, et que $M$ est $I^{\prime}$ agglutiné $\bar{A}^{1}$ de $A$ par $1 \in$ H. Mais $M n^{\prime}$ 'est pas un annérde ( $n i$ un anel), puisque $a c$ et $b d$ ne sont pas addibles dans $M$. C'est-à-dire que $a+b \in \mathbb{M}, c+d \in M$, mais $(a+b)(c+d)=a c+b d \notin M$. Il en résulte, grâce au lemme, que, quelle que soit la graduation commutative et associative $\Delta$ de $A$, une quelconque $\Delta$-graduation $\Gamma$ de $M$ ne jouit pas de l'associativité des scalaires, ce que l'on peut vérifier directement. Reprenons les notations de la démonstration du lemme, en posant $x=1 \in M$. Pour les grades, évidemment non nuls, de $a c$ et $b d \in M$, on a comme précédemnent : $\omega(\mathrm{a} c)=\omega(\mathrm{c})[\omega(\mathrm{b}) \omega(1)]$ et $\omega(\mathrm{b} d)=\omega(\mathrm{b})[\omega(\mathrm{c}) \omega(1)]$. S'il y avait associativité pour $\Gamma$, on en déduirait : $\omega(a c)=[\omega(c) \omega(b)] \omega(1)=[\omega(b) \omega(c)] \omega(1)=\omega(b$ d $)$, d'où une contradiction. Exemple contraire 2 : Voici un annérde $C$, déduit d'un anneau de polynômes, dont la graduation est intrinsèquement non associative. Soit B I'annérde commutatif des monômes sur $\mathbb{Z} /(2)$, en $a, b, c, d, x$ avec les relations $a d=b c=0$. Soit $C$ la plus petite partie de $\bar{B}$, contenant $B$, qui soit un annérde où a $\mathrm{x} \# \mathrm{~b} \mathrm{x}$ et $\mathrm{c} \times \# \mathrm{~d} \mathrm{x}$. Partant de $\mathrm{B}$, on obtient donc $C$ en étendant l'addibilité par ces conditions et par la propriété fondamentale. on montre que (les entiers sont positifs) : $\forall i, j, k, 1, m$ vérifiant $1 \leq i+j=k+1 \leq m$, on a $a^{i} b^{j} x^{m} \# a^{k} b^{l} x^{m}$ et de même avec $c$ et $d$; $\forall i, j, m$ vérifiant $1 \leqslant i+j \leqslant m$, on a $a^{i} c^{j} x^{m} \not b^{i} d^{j} x^{m}$. Ce sont, dans le passage de $\mathrm{B}$ à $\mathrm{C}$, les seules extensions de l'addibilité. Par contre, a $c x$ et $b d x$ ne sont pas addibles, ni $a^{i} c^{j} x^{m}$ et $b^{i} d^{j} x^{m}$ si $m<i+j$. Soit $\Gamma$ une graduation de C. Il est clair que la loi commutative sur $\Gamma n$ 'est pas associative; a $c x$ et $b d x$ étant non nuls et non addibles, 
on a en effet :

$\omega(a)[\omega(x) \omega(d)]=\omega(a) \omega(x . d)=\omega(a) \omega(x c)=\omega(a \times c) \neq \omega(b \times d)=$ $=\omega(b x) \omega(d)=\omega(a x) \omega(d)=[\omega(a) \omega(x)] \omega(d)$.

$M$ et $M_{1}$ étant toujours des monels sur un $_{S}$ anel A (commutatif), et $f: M \rightarrow M$, un quasi-morphisine, soit encore $\xi=\sum_{i=1}^{S} x_{i}$ un élément de $\bar{i}$.

$\xi$ sera dit agglutiné large par $f$ si la somme formelle $\sum_{i=1}^{s} f\left(x_{i}\right)$ après réduction des termes addibles dans $M_{1}$, est un élément de $M_{1}$, que $I$ 'on notera $f(\xi)$. On sait que $l$ 'expression de $\xi$ (avec les $x_{i}$ non addibles entre eux) est unique modulo 0 . si donc $\xi=\sum_{i=1}^{s}\left(x_{i}+e_{i}\right)$, avec $\forall i, e_{i} \in \mathcal{O}$, est une autre

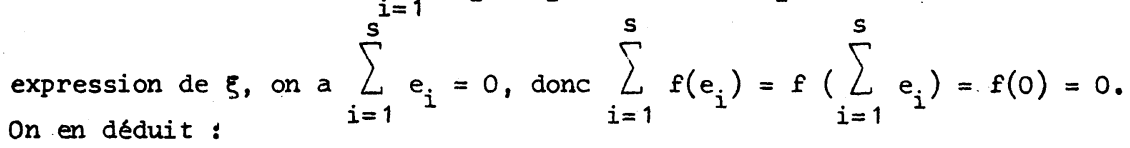

$\sum_{i=1}^{s} f\left(x_{i}+e_{i}\right)=\sum_{i}\left[f\left(x_{i}\right)+f\left(e_{i}\right)\right]=\sum_{i} f\left(x_{i}\right)+\sum_{i} f\left(e_{i}\right)=\sum_{i} f\left(x_{i}\right)$. Donc, $f(\xi)$ ne dépend pas de l'expression de $\xi$. On note $\Lambda^{f}$ l'ensemble de ces agglutinés larges, et on appelle $\Lambda^{f}$ I'agglutiné large de $M$ par $f . D^{\prime}$ abord, $\forall a \in A$ et $\forall \xi \in \Lambda^{f}$, il est clair que a $\xi \in \Lambda^{f}$. Donc $\Lambda^{f}$ est une partie de $\bar{M}$, stable par le produit par les éléments de $A$. Deux éléments $\xi$ et $\eta$ de $\Lambda^{f}$ seront dits addibles dans $\Lambda^{f}$ si et seulement si $f(\xi)$ et $f(\eta)$ sont addibles dans $M_{1}$; alors, $\xi+\eta$ s'obtiendra par juxtaposition, puis réduction des termes addibles dans M. Ces deux opérations font alors de $\Lambda^{f}$ un A-monel. Le milieu de $\Lambda^{f}$ est l'ensemble des $\xi \in \Lambda^{f}$ tels que $f(\xi) \in G_{1}$. Les axiomes de $A$-monel se vérifient pour $\Lambda^{f}$ encore plus aisément que pour $\bar{M}^{f}$; en effet, on raisonne, pour les questions d'addibilité, sur l'élément $f(\xi)$ de $M_{1}$ et non sur les termes $f\left(x_{i}\right)$. Enfin, une étude analogue, faite sur un quasi-morphisme d'anels $f: A \rightarrow B$, conduit à un agglutiné large qui est un anel.

Dans l'exemple où $M$ est un monel sur un anel A (commutatif), où on fixe $x \in M$ (resp. $A$ ) et où $f$ est le quasi-morphisme de $A$-monels $A \rightarrow M$ (resp. $A \rightarrow A$ ) défini par $f(a)=a x, l$ 'agglutiné large sera noté $\Lambda^{x}$. Alors, dans les conditions du corollaire $1, \Lambda^{\mathrm{x}}$ est un anel. Si, de plus, A est un 
annérde, et si $x$ non nul dans $A$ est ni diviseur, ni pseudo-diviseur de 0 , alors $\Lambda^{\mathbf{X}}$ est un annérde.

Diviseurs d'un élément. Plaçons-nous, pour simplifier l'exposé, dans le cas d'un anel unitaire $A$. Soient $x, b \in A, x \neq 0$. Nous dirons que $x$ est diviseur (resp. pseudo-diviseur, resp. diviseur large) de b, s'il existe un élément $\gamma \in A$ (resp. $\gamma \in \bar{A}^{x}$, resp. $\gamma \in \Lambda^{x}$ ) tel que $b=\gamma x$. Il sera dit pseudo-diviseur (resp. diviseur large) propre, si de plus $\gamma \notin \mathrm{A}$ (resp. $\gamma \in \vec{A}^{\mathbf{X}}$ ). De là les notions de diviseur de 0 , de pseudo-diviseur de 0 , de diviseur large de 0. De là aussi les notions d'élément inversible, pseudo-inversible, inversible large.

L'anel unitaire $A$ est dit intègre si, pour tout élément $c$ non nul de A, 1 'homothétie de rapport $c$ est injective. L'anel unitaire $A$ est dit un quasidomaine $s^{\prime} i l n^{\prime} a$ pas de diviseur de 0 . Il est appelé domaine $s^{\prime} i l n^{\prime} a$ de diviseur de 0 d'aucune sorte.

b) Anels et monels intermédiaires. Factorisation d'un quasi-morphisme

Revenons au cas où $\mathbf{f}: \mathbf{M} \rightarrow M_{1}$ est un quasi-morphisme de monels. Que I'on considère $M$ comme partie de $\vec{M}^{f}$ ou de $\Lambda$, ses éléments ont la même addibilité. Et $\Lambda^{f}$ ne diffère en quelque sorte de $\bar{M}$ que par un agrandissement du milieu (et par l'agrandissement corrélatif du A-monel). Si donc $\Lambda^{f}$ est différent de $\vec{M}$, il ne peut etre un simple agglutiné $\vec{M}^{h}$. Lorsque $f$ est donnée, $I^{\prime}$ ensemble des A-monels intermédiaires entre $M$ et $\bar{M}$, et dont $I^{\prime}$ addibilité induit sur les éléments de $M l$ 'addibilité par $f$, admet $\vec{M}$ comme plus petit élément. Inversement, pour l'ensemble des A-monels, intermédiaires entre $M$ et $\bar{M}$, dans lesquels les éléments de $M$ ont une même addibilité donnée, il existe un plus petit élément : on prend I'un P de ces A-monels et l'injection canonique $j: M \rightarrow P$; on voit que $\bar{M}^{j}$ est ce plus petit élément. Ces agglutinés simples $\overrightarrow{\mathrm{M}}$ par un quasi-morphisme, seront aussi appelés les petits intermédiaires entre $M$ et $\bar{M}$. Notons que $M$ et $\bar{M}$ en sont, le premier par 1 'identité de $M$, le second par $I$ 'injection canonique de $M$ dans $\bar{M}$.

En particulier, si A est un anel unitaire, tout (A-monel) petit intermédiaire entre $A$ et $\bar{A}$ est un agglutiné $\bar{A}^{f} ;$ (f quasi-morphisme de $\left.A-m o n e l s\right)$ il est même un agglutiné $\vec{A}^{x}$, en posant $x=1 \in \vec{A}^{f}$ (il n'est pas, en général, un $\bar{A}^{y}$, avec $y \in A$ ). Il en résulte que tout petit interinédiaire qui est un anel se trouve parmi les $\vec{A}^{X}$ précédents. Mais nous avons vu plus haut qu'un $\vec{A}^{\mathbf{x}}$ 
n'est pas forcément un anel, nême si la graduation de A est associative.

Lemme 2 : (a) Soit $f: M \rightarrow M$, un quasi-morphisme de A-monels. Il existe un unique homomorphisme de A-monels, $f^{\prime}: \bar{M}^{f} \rightarrow M_{1}$, prolongeant $f$.

(b) Soit $f: A \rightarrow B$ un quasi-morphisme d'anels.

Il existe un unique homomorphisme d'anels $f^{\prime}: \bar{A}^{f} \rightarrow B$, prolongeant $f$.

Tout $\xi \in \bar{M}^{f}$ (resp. $\bar{A}^{f}$ ) s'écrit $\xi=\sum_{i=1}^{s} x_{i}$, où les $f\left(x_{i}\right)$ sont mutuellement addibles, mais non les $x_{i}$. On pose nécessairement $f^{\prime}(\xi)=\sum_{i=1}^{s} f\left(x_{i}\right)$, ce qui est indépendant de $I^{\prime}$ expression de $\xi$ (on $I^{\prime} a$ vu pour la définition de $\Lambda^{f}$ ). Il est clair que $f^{\prime}$ prolonge $f$, est unique, et est un quasi-morphisme. Enfin, si $\xi$ et $\eta=\sum_{j=1}^{r} y_{j} \in \bar{M}^{f}$ (resp. $\bar{A}^{f}$ ) sont tels que $f^{\prime}(\xi) \# f^{\prime}(\eta), \quad f^{\prime}(\xi) \notin \mathcal{O}_{1}, f^{\prime}(\eta) \notin \mathcal{O}_{1}$, il est clair que $I^{\prime}$ addibilité (dans $M_{1}$, resp. B) se transmet par $f^{\prime}(\xi)$ et $f^{\prime}(\eta)$ vers $f\left(x_{i}\right)$ et $f\left(y_{j}\right)$. Donc $\xi$ et $\eta$ sont addirles dans $\bar{M}^{f}$ (resp. $\bar{A}^{f}$ ), et $f^{\prime}$ est un homomorphisme.

En particulier, un quasi-morphisme $f: M \rightarrow M_{1}$ de $A-$ monels se prolonge de manière unique en un homomorphisme $\bar{f}: \bar{M} \rightarrow \bar{M}$, de $\bar{A}$-modules. Il suffit de prendre $\overline{\mathrm{f}}=(\mathrm{u} \circ \mathrm{f})^{\prime}$ où $\mathrm{u}$ est $\mathrm{I}^{\prime}$ injection canonique $\mathrm{M}_{1} \rightarrow_{\mathrm{M}_{1}}$. Un quasi-morphisme d'anels se prolonge de meme en un homomorphisme d'anneaux.

Ce lemme montre que tout quasi-morphisme se factorise canoniquement en $f=f^{\prime} \circ j$, ou $j$ est $I^{\prime}$ injection canonique $M \rightarrow \bar{M}^{f}\left(\right.$ resp. $A \rightarrow \bar{A}^{f}$ ). Il est maintenant clair que $I^{\prime}$ agglutiné large $\Lambda^{f}$ est l'agglutiné simple de $\bar{M}^{f}$ (resp. $\bar{A}^{f}$ ) par $f^{\prime}$. On sait déjà que cette seconde agglutination se borne à "étendre le milieu" du A-monel $\bar{M}^{f}$ (resp. de l'anel $\bar{A}^{f}$ ).

En particulier, si $\mathrm{A}$ est un anel d'anneau $\overline{\mathrm{A}}$ (resp. $\mathrm{M}$ un A-monel de $A$-module $\bar{M}$ ), et si $B$ est un anel intermédiaire entre $A$ et $\bar{A}$ (resp. $M_{1}$ un A-monel intermédiaire entre $M$ et $\bar{M}$ ), on déduit $B$ de $A$ (resp. $M$, de $M$ ) grâce au plus à deux agglutinations simples : la première par l'injection canonique $f: A \rightarrow B\left(\right.$ resp. $\left.M \rightarrow M_{1}\right)$, la seconde par $f^{\prime}$. On obtient donc ainsi, par au plus deux agglutinations simples, tout anel (resp. A-monel) intermédiaire.

Comme application, soit $\mathrm{A}$ un anel (commutatif) unitaire, et soit

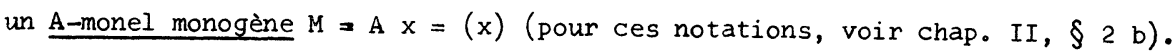


Le quasi-morphisme $f: A \rightarrow M=A x$ défini par $f(a)=a x$ est en général non surjectif. Il se prolonge en un homomorphisme $f^{\prime}: \vec{A}^{X} \rightarrow M$, qui se prolonge à son tour en un homomorphisme surjectif $f^{\prime \prime}: \Lambda^{x}=\overline{\bar{A}}^{x^{\prime}} \rightarrow M=A x$. Par définition

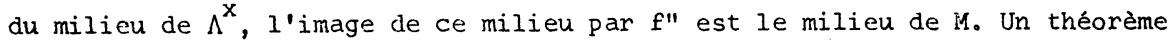
d'isomorphie (vu ultérieurement) montre alors que $A \mathrm{x}=M$ est isomorphe à un quotient du A-monel $\Lambda^{X}$ par un sous-monel. S1, de plus, une graduation de $A$ est associative, et si $\omega(x)$ associe les scalaires dans une graduation de $M$, $M=A x=\Lambda^{x} \times$ sera un $\Lambda^{x}$-monel, isomorphe (comme $\Lambda^{x}$-monel) à un quotient de $I^{\prime}$ anel $\Lambda^{\mathrm{X}}$ par un idéal. Ces résultats $\mathbf{s}^{\prime}$ appliquent évidemment au cas d'un moduloide monogène sur un annélde unitaire.

Soient A un anel, et B un agglutiné de A qui soit un anel. Il est clair que pour $B$ les notions d'idéal et de sous-A-monel se confondent. Soit $\bar{A}$ (resp. $\bar{M}$ ) un anneau (resp. un $\bar{A}$-module) quasi-gradué d'ensemble homogène $A$ (resp. $M$ ). Un homomorphisme quasi-homogène produit un changement de la structure quasi-graduée : l'anneau (resp. le module) abstrait reste le même, mais l'ensemble homogène s'étend (des éléments nouveaux s'homogénélsent), et la quasi-graduation change, avec un groupement des grades.

Dans 1 'anneau quasi-gradué $\bar{A}$ (d'ensemble homogène $A$ ) la multiplication par un élément $x$ homogène $d^{\prime} u n \bar{A}$-module $\bar{M}$, quasi-gradué, conduit à considérer $\bar{A}$ comme un $\overline{\mathrm{A}}$-module quasi-gradué dont $\mathrm{l}^{\prime}$ ensemble homogène est $\overline{\mathrm{A}}^{\mathrm{X}} \supset \mathrm{A}$. $\mathrm{Si}$, de plus, il y a associativité d'une graduation de $\bar{M}$, l'ensemble $\bar{A}^{\mathbf{X}}$ des nouveaux éléments homogènes est multiplicativement stable, d'où pour $\bar{A}$ une nouvelle structure d'anneau quasi-gradué. Inversement, toute quasi-graduation de I'anneau abstrait $\bar{A}$, pour laquelle l'ensemble homogène contient $A$, ou bien possède pour ensemble homogène un tel $\vec{A}^{\mathbf{X}}$, ou un anel qui s'en déduit par une extension du milieu de $\vec{A}^{\mathbf{x}}$.

c) Agglutinés par une partie multiplicative de A. Divisions des idéaux : Outre les agglutinés des types précédents $\left(\bar{M}^{f}\right.$ et $\left.\Lambda^{f}\right)$, il apparart aussi des ensembles d'agglutinés dont la structure est en général plus complexe. Nous allons étudier sommairement deux cas.

Soient $\mathrm{A}$ un anel (commutatif), $M$ un A-monel, et $\mathrm{P}$ une partie non vide de M. Considérons les ensembles : $T=T(A, P)=\bigcap_{x \in P} \bar{A}^{x}=\left\{\gamma=\sum_{i=1}^{S} c_{i} \in \bar{A} ; \forall x \in P, \forall i\right.$ et $\left.j, c_{i} x \not c_{j} x\right\}$ et $\Lambda=\Lambda(A, P)=\bigcap_{\mathrm{X} \in \mathrm{P}} \Lambda^{\mathrm{X}}$. Ces ensembles sont stables par multiplication par les éléments de A. Deux éléments $\gamma$ et $\gamma$ 'de $T$ scront dits addibles dans $T$ si et seulement s'ils le sont dans $\bar{A}^{\mathrm{x}}, \forall x \in P$; la somme est définie de 
manière évidente. Dans $T$, les axiomes suivants des A-monels sont vérifiés : $A, B_{1}, B_{2}$, les distributivités et la propriété fondamentale. Mais on a une structure plus complexe que celle de A-monel. On trouve dans $T$ deux parties $\mathcal{O}$ et $\mathcal{O} \cdot$ telles que $(0) \subset \mathcal{O} \subset \mathcal{G}^{\prime} \subset \mathrm{T}$, que $\mathcal{O}$ est $I^{\prime}$ ensemble des éléments de $T$ addibles à tout dans $T$, et que $\mathcal{O}^{\prime}$ est la plus grande partie de $T$ pour laquelle les éléments de T.. O' transmettent l'addibilité. On considère l'ensemble $E \subset P(T)$ des parties non vides de $T$ formées d'éléments mutuellement addibles. $E$ étant inductif, soit $\mathrm{G}$ un élément maximal. Dans $\mathrm{T}$, la relation $\gamma \not \# \gamma^{\prime}$ implique $\gamma \not \# \gamma+\gamma^{\prime}$, puisque cela est vrai dans chaque $\overline{\mathbf{A}}^{\mathrm{X}}$. Donc $\mathrm{G}$ est stable par I'addition de T. De plus, pour chaque $\mathbf{x} \in \mathrm{P}$, $G$ est une partie d'un groupe d'addibilité de $\vec{A}^{x}$, contient 0 , et contient, avec un élément $\gamma$, son opposé. G est donc un groupe abélien, encore appelé groupe d'addibilité. $\mathcal{O}$ est $l$ 'intersection de ces éléments maximaux. Pour $\Lambda$, une étude analogue en tout point est évidente.

Appliquons ces considérations à la division d'un idéal a d'un anel commutatif $A$ par une partie non vide $b$ de $A$. La notion classique $a: b=\{c \in A ; \forall b \in b, c b \in a\}=\{c \in A ; c b \subset a\}$ donne encore le plus grand idéal $\mathfrak{\Im}$ de $A$ tel que $\Im \mathfrak{b} \subset \mathfrak{a}$. On peut aussi définir deux autres divisions :

$$
\begin{aligned}
& a \overline{:} b=\{\gamma \in T(A, b) ; \forall b \in b, \gamma b \in a\} \\
& a \overline{:} b=\{\gamma \in \Lambda(A, b) ; \forall b \in b, \gamma b \in a\} \text {. Ces deux parties de } \bar{A}
\end{aligned}
$$
sont stables par multiplication par les éléments de A. Mais ce qui précède montre qu'en général leur structure ne sera pas assez simple pour être commode. Notons cependant que $b \supset b^{\prime}$ implique que le quotient de $a$ par $b$ est contenu dans celui de a par b' (quelle que soit la division envisagée). On a des considérations analogues pour la division d'un sous-A-monel d'un nonel $M$, par une partie non vide de $M$.

Soient $A$ un anel (commutatif), $M$ un A-monel, et $S$ une partie non vide, multiplicativement stable de A. On appellera agglutiné de M par S la partie de $\bar{M}$ définie par $\bar{M}^{S}=\left\{\xi \in \bar{M} ; \exists s \in s, \xi \in \bar{M}^{s}\right\}=U \bar{M}^{s}$, qui évidemment est stable par multiplication par les éléments de A et qui est munie de $\underline{I}$ 'addibilité par $\mathrm{S}$ ainsi définie : $\xi$ et $\eta \in \overline{\mathrm{M}}^{\mathrm{S}}$ sont addibles par $\mathrm{S}$ si et seulement s'il existe $t \in S$ tel que, $\forall i$ et $j, t x_{i} \not t y_{j} ; l a$ somme se calcule alors dans $\overline{\mathrm{M}}$. Dire que $\xi$ et $\eta \in \overline{\mathrm{M}}^{\mathrm{S}}$ sont addibles par $\mathrm{S}$, grace à $t$, équivaut à dire qu'ils sont éléments addibles d'un même agglutiné simple $\bar{M}^{\sigma}$, avec $\sigma \in S$. En effet, si $\xi \in \bar{M}^{s}$ et $\eta \in \bar{M}^{r}$ sont addibles par $s$ 
grâce à $t$, on le voit en posant $\sigma=r s t$. (Par contre, si on ne supposait pas $S$ multiplicativement stable, il pourrait arriver que deux tels éléments $\xi$ et $\eta \in \bar{M}^{S}$ soient addibles par $S$, sans appartenir à un même agglutiné simple $\bar{M}^{\mathrm{u}}, u \in S$; et $\xi+\eta$ pourrait n'être pas dans $\left.\bar{M}^{S}\right)$. Il est facile

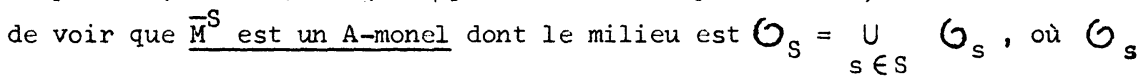
est le milieu de $\bar{M}^{s}$. En effet, quel que soit $\xi \in G_{S}$, il existe $s \in s$ tel que les $s x_{i}$ appartiennent au milieu $\mathcal{O}$ de $M$; quel que soit $\eta \in \bar{M}^{S}$, il existe $r \in S$ tel que les $r y_{j}$ soient addibles entre eux, donc aussi les s $r y_{j}$; comme $r s x_{i} \in \mathcal{O}, \forall i\left(\right.$ axiome $\left.D_{5}\right), \xi$ et $\eta$ sont des éléments addibles de $\bar{M}^{r} \mathrm{~s}$. Soient $\xi$, $\eta$ et $\zeta \in \overline{\mathrm{M}}^{\mathrm{S}}$, avec $\eta \notin \mathcal{O}_{\mathrm{S}}, \xi$ et $\eta$ étant addibles par $\mathrm{s}$, de même que $\eta$ et $\zeta$. Il existe $u$ et $v \in S$ tels que $\xi$ et $\eta$ soient éléments addibles de $\bar{M}^{\mathrm{u}}$, et $\eta$ et $\zeta$ de $\overline{\mathrm{M}}^{\mathrm{v}}$. Alors, $\xi$ et $\eta$ (resp. $\eta$ et $\zeta$ ) sont éléments addibles de $\overline{\mathrm{M}}^{\mathrm{u}} \mathrm{v}$, avec $\eta \notin \mathcal{O}_{\mathrm{u} \mathrm{v}}$; donc $\xi$ et $\zeta$ sont addibles dans $\overline{\mathrm{M}}^{\mathrm{u} v}$, et aussi dans $\overline{\mathrm{M}}^{\mathrm{S}}$. La vérification des autres axiomes des A-monels, pour $\overline{\mathrm{M}}^{\mathrm{S}}$, est alors immédiate.

On a les résultats analogues pour la réunion $\Lambda^{S}$ des agglutinés larges $\Lambda^{s}(s \in S)$ de $M$.

Soient $A$ un anel (commutatif) et $S$ une partie non vide, multiplicativement stable de $A$. Le $A-$ monel $\bar{A}^{-S}$ (resp. $\Lambda^{S}$ ) est un anel (même lorsque la graduation $n$ 'est pas associative). Soient $\xi, \eta \in \bar{A}^{-S}$; donc il existe $s, 2 \in S$ tels que $\xi \in \bar{A}^{s}$ et $\eta \in \bar{A}^{t}$; alors $(s t)(\xi \eta)=(s \xi)(t \eta)$ montre, avec la propriété fondamentale dans $A$, que $\xi \eta \in \bar{A}^{\text {st }}$; donc $\bar{A}^{S}$ est multiplicativement stable. Soit $\eta \in \bar{A}^{t}$ et soient $\xi$ et $\xi^{\prime}$ deux éléments addibles de $\bar{A}^{S}$, donc éléments addibles d'un $\bar{A}^{S}$ (avec $s, t \in S$ ); la propriété fondamentale dans $A$ montre que $(s t)(\eta \xi)=(t \eta)(s \xi) \#(t \eta)\left(s \xi^{\prime}\right)=(s t)\left(\eta \xi^{\prime}\right)$, donc que $\eta \xi$ et $\eta \xi^{\prime}$ sont éléments addibles de $\bar{A}^{-s t}$. Les autres vérifications sont triviales.

d) Agglutination par un grade :

Soient $A$ un anel (commutatif) de graduation $\Delta$ (commutative), et $M$ un A-monel (éventuellement $A$ ) ayant une $\Delta$-graduation $\Gamma$ (éventuellement $\Delta$ ). Les éléments $d_{1}, \ldots . ., d_{p}$ de $\Delta$ seront dits agglutinés par le grade $\gamma \in \Gamma$ si des $d_{i} \gamma$ ont la même valeur. Cela s'appelle l'agglutination dans $\Delta$ par un grade $\gamma$. On peut considérer de même l'agglutination dans $\Gamma$ par un grade $a \in \Delta$. Définissons l'agglutination dans A par un grade.

Définition. Lemme 3 : Soient $A$ un anel de graduation $\Delta$ et de milieu $\Omega, \xi$ un élément de $\Delta$, et e un entier $\geq 1$. (1) Soit a $\in A$. Si a $\notin$ Ret si pour toute factorisation $a=f_{1} \ldots f_{q}$ (les $\left.f_{j} \in A\right)$, le produit 
$\omega\left(f_{1}\right) \ldots \omega\left(f_{q}\right) \xi \ldots . \xi$ (e facteurs $\xi$ ) est associatif et non nul, sa valeur $\omega(a) \xi^{e}$ est par définition le grade $\omega^{\prime}(a)$ dans l'agglutiné qui suit. Dans tous les autres cas, on pose $\omega^{\prime}(a)=0$. (2) Soit $\bar{A}^{\xi}$, e la partie de $\bar{A}$ formée des $\alpha=\sum_{i=1}^{n} a_{i}$ tels que les $\omega^{\prime}\left(a_{i}\right)$ non nuls aient la même valeur, qui sera le grade $\omega^{\prime}(\alpha)$; on pose $\omega^{\prime}(\alpha)=0$ si tous les $\omega^{\prime}\left(a_{i}\right)$ sont nuls. $\bar{A}^{\xi}, e$ est un A-monel, appelé l'agglutiné de $A$ par $\xi, e$, et $\Delta$ en est une $\Delta$-graduation ; $\omega^{\prime}(a)$ est donc le $\Delta$-grade de $a_{\text {. }}$ On convient que $\bar{A}^{\xi}, 0$ désigne $I$ 'anel $A$, et $\bar{A}^{\xi}$ le monel $\vec{A}^{\xi}, 1$. On a $\boldsymbol{\omega}^{\circ}(a)=0$ dans les trois cas suivants : ou bien a $\in \Omega$. ou bien a $\notin \Omega$, et, pour toute factorisation $a=f_{1} \ldots f_{q}$ de $a, \omega\left(f_{1}\right) \ldots \omega\left(f_{q}\right) \xi \ldots \xi$ est associatif et nul. Ou bien a $\$ \Omega$, et il existe une factorisation $a=f_{1} \ldots f_{q}$ telle que $\omega\left(f_{q}\right) \ldots \omega\left(f_{q}\right) \xi \ldots \xi$ ne soit pas associatif (cette non-associativité provient d'un produit de trois facteurs dont l'un contient nécessairement $\xi$, puisque a $\notin \Omega$. Noter aussi que $\xi \ldots . . . \xi$ peut ne pas être associatif).

On peut montrer que, si un produit donné $p=\omega\left(f_{q}\right) \ldots \omega\left(f_{q}\right) \xi \ldots \xi \xi$ n'est pas associatif, il existe un triple $\alpha \beta \gamma$, produit extrait du précédent (c'est-à-dire dont les termes $\alpha, \beta, \gamma$ sont des produits de certains facteurs de $p$ ), non-associatif, et dont l'un des facteurs est $\xi$. Dans un triple $\alpha \beta \gamma$ extrait de $p$, appelons longueur $(\geqslant 3$ ) le nombre des facteurs du produit $p$ qui y figurent. Lorsque $p n^{\prime}$ est pas associatif, prenons parmi les triples nonassociatifs extraits de $p$, un triple $\alpha \beta \gamma$ de longueur minimale $m$. Si $\gamma=\gamma \cdot \gamma$ ", supposons que les triples $\alpha$ ( $\left.\beta \gamma^{\prime}\right) \gamma^{\prime \prime}$ et $(\alpha \beta) \gamma^{\prime} \gamma^{\prime \prime}$ soient associatifs. Les triples $\beta \gamma^{\prime} \gamma^{\prime \prime}$ et $\alpha \beta \gamma^{\prime}$ sont de longueur strictement inférieure à $m$, donc sont associatifs. On a alors : $(\alpha \beta)\left(\gamma^{\prime} \gamma^{\prime \prime}\right)=\left[(\alpha \beta) \gamma^{\prime}\right] \gamma^{\prime \prime}=\left[\alpha\left(\beta \gamma^{\prime}\right)\right] \gamma^{\prime \prime}=\alpha\left[\left(\beta \gamma^{\prime}\right) \gamma^{\prime \prime}\right]=\alpha\left[\beta\left(\gamma^{\prime} \gamma^{\prime \prime}\right)\right]$. Donc, le triple $\alpha \beta$ ( $\gamma \cdot \gamma ")$ serait associatif, une contradiction. Il en résulte que si $\alpha \beta\left(\gamma^{\prime} \gamma^{\prime \prime}\right)$ est un triple, extrait de $p$, non associatif de longueur minimale, il en existe un autre (soit $\alpha\left(\beta \gamma^{\prime}\right) \gamma^{\prime \prime}$, soit $(\alpha \beta) \gamma^{\prime} \gamma^{\prime \prime}$ ) de même longueur, où le dernier terme $\gamma=\gamma \cdot \gamma$ " est remplacé par.son sous-facteur $\gamma "$ (noter que tout est associatif dans le produit $\gamma$ qui est de longueur $\leq m-2)$. En particulier, si $\xi$ figure dans le terme $\gamma$ du triple non associatif $\alpha \beta \gamma$, il existe un triple non associatif de meme longueur $\alpha, \beta, \xi$. Si, dans le triple non associatif minimal $\alpha \beta \gamma$, le facteur $\xi$ figure dans $\beta$ seulement, un raisonnement analogue conduit à un triple non associatif minimal où l'un des termes (au milieu ou à une extrémité) est $\xi$. 
Notons le cas singulier ou $\xi \ldots \ldots \ldots$ (pour $e \geq 4$ ) ne serait pas associatif, et où, par suite, $\bar{A}^{\bar{\zeta}, e}=\bar{A}$.

organisons $L=\bar{A}^{\bar{y}}$,e en A-monel. Posons que $\alpha=\sum_{i} a_{i}$ et $\beta=\sum_{j} b_{j}$ sont addibles dans $L$ si et seulement si, quels que soient $i$ et $j$, les $\omega^{\prime}\left(a_{i}\right)$ et $\omega^{\prime}\left(b_{j}\right)$ non nuls sont la même valeur, la somme se calculant alors dans $\bar{A}$. Le milieu $\mathcal{O}$ de $L$ sera l'ensemble des $\alpha=\sum_{i} a_{i}$ tels que $\omega^{\prime}\left(a_{i}\right)=0$ pour tout $i$. On fait le produit $c \boldsymbol{\alpha}$ de $\alpha \in \mathrm{L}$ par $\mathrm{c} \in \mathrm{A}$ comme dans le A-monel $\bar{A}$. Remarquons que, grâce à la définition de $\omega^{\prime}$, pour c et a $\in A$, si $\underline{\omega^{\prime}(c a) \neq 0}$, pour toute factorisation $c a=g_{1} \ldots . g_{q}$, il y a associativité de $\omega\left(g_{1}\right) \ldots \omega\left(g_{q}\right) \quad \xi \ldots \ldots . . \xi$, et $l$ 'on $a$ :

$\omega^{\prime}(c a)=\omega(c a) \xi^{e}=\omega(c) \omega(a) \xi^{e}=\omega(c) \omega^{\prime}(a)$. Donc, d'une part c $\alpha$ est dans $L$, et d'autre part $\Delta\left(\right.$ ou $\left.\Delta \xi^{\mathrm{e}}\right)$ est une $\Delta$-graduation de $L$, et il est cohérent d'appeler $\omega^{\prime}(\alpha)$ un grade. Si $\alpha=\sum_{i} a_{i}, \beta=\sum_{j} b_{j}$ et $\alpha^{\prime}=\sum_{k} a_{k}^{\prime}$ sont éléments de $\mathrm{L}$, si dans $\mathrm{L}$ on a $\alpha \# \beta \# \alpha^{\prime}$, et si $\beta \notin \mathcal{O}$, il existe un terme $b_{j 0}$ de $\beta$ tel que $\omega^{\prime}\left(b_{j o}\right) \neq 0$; donc les grades $\omega^{\prime}\left(a_{i}\right)$ et $\omega^{\prime}\left(a_{k}^{\prime}\right)$ non nuls sont égaux à $\omega^{\prime}\left(b_{j o}\right)$ d'où $\alpha \not \alpha^{\prime}$ (axiome $\left.B_{4}\right)$. Soient $c \in A$, et $\alpha$ et $\beta$ deux éléments addibles de $L ;$ les $\omega^{\prime}\left(a_{i}\right)$ et $\omega^{\prime}\left(b_{j}\right)$ non nuls sont égaux. Si $\omega^{\prime}(c \alpha)$ et $\omega^{\prime}(c \beta)$ sont non nuls, donc respectivement égaux à un $\omega^{\prime}\left(c a_{i}\right) \neq 0$ et à un $\omega^{\prime}\left(\begin{array}{cc}c & b_{j}\end{array}\right) \neq 0$, la remarque précédente montre que : $\omega^{\prime}\left(c a_{i}\right)=\omega(c) \omega^{\prime}\left(a_{i}\right)=\omega(c) \omega^{\prime}\left(b_{j}\right)=\omega^{\prime}\left(c b_{j}\right)$. Donc $c \alpha$ et $c \beta$ sont addibles (axiome $D_{1}$ ). Soient $c$ et $d \in A, c \# d$, et $\alpha \in L$. La remarque montre que si, pour un $i, \omega^{\prime}\left(c a_{i}\right) \neq 0$, on a $\omega^{\prime}\left(c a_{i}\right)=\omega(c) \omega^{\prime}(\alpha)$. si $\omega^{\prime}(c \alpha)$ et $\omega^{\prime}(d \alpha)$ sont non nuls, on déduit d'abord que $\omega(c)$ et $\omega(d)$ sont non nuls, donc égaux; ensuite $q u$ 'au moins un terine $c a_{i}$ (resp. $d a_{j}$ ) est tel que $0 \neq \omega^{\prime}\left(c a_{i}\right)=\omega^{\prime}(c) \omega^{\prime}\left(a_{i}\right)=\omega(d) \omega^{\prime}\left(a_{j}\right)=\omega^{\prime}\left(d a_{j}\right)$. Donc c $\alpha$ et c $\beta$ sont addibles (axiome $D_{3}$ ). La vérification des autres axiomes est immédiate, et $\mathrm{L}=\overline{\mathrm{A}}^{\bar{\xi}}$,e est bien un $\mathrm{A}$-monel intermédiaire entre $\mathrm{A}$ et $\overline{\mathrm{A}}$.

Il faut bien noter que $\bar{A}^{\bar{A}, e} n^{\prime}$ est pas forcément un anel : Dans I'exemple contraire 2 (suite au lemme 1), prenons l'annéide $C$, et considérons I'agglutiné $L=\bar{C}^{\omega(x)}$, en notant $\omega(x)$ le grade de $x$ dans une graduation de $C$. Alors, $a+b$ et $c+d$ sont dans $L$, mais leur produit $a c+b d n ' y$ est pas. 
Lemme 4 : Soit $A$ un anel de graduation $\Delta, \xi$ un élément de $\Delta$, et e un entier $\geqslant 1$. Si, dans $\Delta$, tout produit de trois termes, dont I'un, extérieur, est $\xi$, est associatif (on dira que $\xi$ associe, dans $\Delta$ ), $\bar{A}^{\xi}, e$ est un anel de milieu $\underset{d \in \Delta^{\prime}}{\oplus} A_{d}$, où $\Delta^{\prime}$ est la partie de $\Delta$ annulée par $\xi^{e}$. (cet anel pourra être noté $\bar{A}^{\xi^{e}}$ ).

Notons $\Gamma=[\omega(A), \xi]$ la partie de $\Delta$ engendréc par $\omega(A)$ et $\xi$, C'est-à-dire la plus petite partie stable de $\Delta$, contenant $\omega(A)$ et $\xi$. Une condition suffisante pour le lenme 4 est que tout produit de trois termes de $\Gamma$, dont l'un extérieur est $\xi$, soit associatif. Alors, on dira que $\xi$ associe extérieurement dans $\Gamma$, et cela implique, pour tout entier $i \geq 1$, que $\xi^{i}$ (qui est alors un produit associatif) associe dans $\Gamma$, extérieurement et intérieurement. Montrons-le. $D^{\prime}$ abord, si $\lambda \in \Gamma$ associe dans $\Gamma$ extérieurement, la commutativité montre qu'il associe intérieurement ; on a (avec $\alpha, \beta \in \Gamma$ ) $(\alpha \lambda) \beta=\beta(\alpha \lambda)=\left(\begin{array}{ll}\beta & \alpha\end{array}\right) \lambda=\left(\begin{array}{ll}\alpha \beta & \beta\end{array}\right) \lambda=\alpha\left(\begin{array}{ll}\beta & \lambda\end{array}\right)=\alpha(\lambda \beta)$. Ensuite, si $\xi$ et $\xi^{i}$ associent dans $\Gamma$, on a (avec $\alpha, \beta \in \Gamma$ ) : $(\alpha \beta) \xi^{i+1}=\left[(\alpha \beta) \xi^{i}\right] \xi=\left[\alpha\left(\beta \xi^{i}\right)\right] \xi=\alpha\left[\left(\beta \xi^{i}\right) \xi\right]=\alpha\left[\beta\left(\xi^{i} \xi\right)\right]=\alpha\left(\beta \xi^{i+1}\right)$, ce qui montre que $\xi^{i+i}$ associe dans $\Gamma$.

Montrons le lemme. On a $\mathcal{O}=\left\{\alpha \in \bar{A}^{\bar{\xi}, e} ; \forall i, \omega\left(a_{i}\right) \xi^{e}=0\right\}$, donc le milieu du $A$-monel $L=\bar{A}^{\xi}$,e est celui annoncé. Restent à vérifier les axiomes $A$ et $D$ des anels, ce qui est immédiat, sauf peut-être pour les suivants : Si $\alpha$ et $\beta \in L$, étudions $\alpha \beta=\sum_{i, j} a_{i} b_{j}$. Lorsque $i \neq i_{1}$ et $j \neq j_{1}$, et que $\omega^{\prime}\left(a_{i} b_{j}\right)$ et $\omega^{\prime}\left(a_{i}, b_{j}\right)$ sont non nuls, on a évidemment $\omega^{\prime}\left(a_{i}\right)=\omega^{\prime}\left(a_{i_{1}}\right) \neq 0, \omega^{\prime}\left(b_{j}\right)=\omega^{\prime}\left(b_{j_{1}}\right) \neq 0$, et enfin $\omega^{\prime}\left(a_{i} b_{j}\right)=\omega\left(a_{i}\right) \omega^{\prime}\left(b_{j}\right)=\omega\left(a_{i}\right) \omega^{\prime}\left(b_{j}\right)=\omega\left(a_{i}\right)\left[\omega\left(b_{j}\right) \xi^{e}\right]=$ $=\omega\left(b_{j_{1}}\right)\left[\omega\left(a_{i}\right) \xi^{e}\right]=\omega\left(b_{j}\right) \omega^{\prime}\left(a_{i}\right)=\omega\left(b_{j_{1}}\right) \omega^{\prime}\left(a_{i_{1}}\right)=\omega^{\prime}\left(a_{i_{1}} b_{j_{1}}\right)$.

On notera l'intervention de l'associativité par $\xi^{e}$. Donc, $\alpha \beta$ est élément de I (Axiome Ao). Ensuite, soient $\alpha, \alpha^{\prime}, \beta \in \mathrm{L}$ vérifiant $\alpha \# \alpha^{\prime}$. Si deux termes, soient $a_{i} b_{j}$ et $a_{k}^{\prime} b_{l}$, appartenant respectivement aux sommes $\alpha \beta$ et $\alpha^{\prime} \beta$, ont des grades $\omega^{\prime}\left(a_{i} b_{j}\right)$ et $\omega^{\prime}\left(a_{k}^{\prime} b_{l}\right)$ non nuls, on a : $\omega^{\prime}\left(b_{j}\right)=\omega^{\prime}\left(b_{1}\right) \neq 0, \omega^{\prime}\left(a_{i}\right)=\omega^{\prime}\left(a_{k}^{\prime}\right) \neq 0$, et enfin $\omega^{\prime}\left(a_{i} b_{j}\right)=\omega\left(a_{i}\right) \omega^{\prime}\left(b_{j}\right)=\omega\left(a_{i}\right) \omega^{\prime}\left(b_{1}\right)=\omega\left(a_{i}\right)\left[\omega\left(b_{1}\right) \xi^{e}\right]=$ $=\omega\left(b_{1}\right)\left[\omega\left(a_{i}\right) \xi^{e}\right]=\omega\left(b_{1}\right) \omega^{\prime}\left(a_{i}\right)=\omega\left(b_{1}\right) \omega^{\prime}\left(a_{k}^{\prime}\right)=\omega^{\prime}\left(a_{k}^{\prime} b_{1}\right)$. 
(Noter encore l'associativité par $\xi^{e}$ ). Il en résulte l'addibilité de $\alpha \beta$ et de $\alpha^{\prime} \beta$ (axiome $\left.D_{1}\right)$.

Remarques : $1^{\circ}$ ) Si $\xi$ associe dans $\Gamma=[\omega(A), \xi]$ (ou dans $\Delta$ ), le A-monel $\bar{A}^{\xi}, \bar{e}$ se déduit de $A$ : pour le milieu, par l'"adjonction" éventuelle à $\Omega$ de groupes entiers d'addibilité de $A ;$ et pour les groupes d'addibilité, par la "somme" de groupes entiers d'addibilité. On a en effet (voir lenme 3, remarque sur l'associativité) : $\omega^{\prime}(a)=\omega(a) \xi^{e}$, d'où le résultat.

$2^{\circ}$ ) Si $\xi$ associe dans $\Delta, s \xi^{e}=t \xi^{e}$ (avec $s, t \in \Delta$ ) est une relation d'équivalence $R$ dans $\Delta$, compatible avec la loi de $\Delta$. En effet, $\gamma=\xi^{\mathrm{e}}$ associe dans $\Delta$, et les relations $s \gamma=t \gamma, u \gamma=v \gamma$ (avec $u, v \in \Delta$ ) impliquent $(s u) \gamma=s(u \gamma)=s(v \gamma)=(s v) \gamma=v(s \gamma)=v(t \gamma)=(t v) \gamma$. Donc l'ensemble quotient $\Delta / R$, muni de la loi quotient est une graduation de I'anel $\bar{A}^{\xi}, e$. (Attention : $\Delta$ ou $\Delta \xi^{e} n^{\prime}$ en sont pas).

Lemme 5 : Soient $A$ un anel de graduation $\Delta$, et $\xi \in \Delta$.

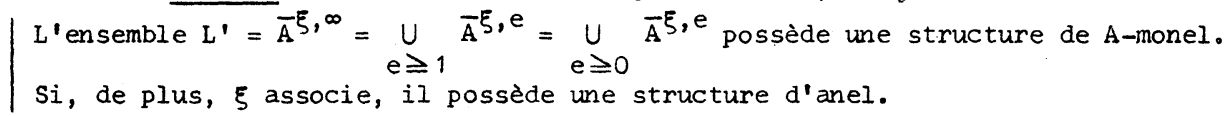
Il est clair que $\bar{A}^{\bar{\xi}, e} \subset \bar{A}^{-\bar{\xi}, e+1}$ et que $\mathcal{O}_{e} \subset \mathcal{O}_{e+1}\left(\mathcal{O}_{e}\right.$ désignant ici le milieu de $\left.\bar{A}^{\xi}, e\right)$. Deux éléments de $L$ ' seront dits addibles dans $L^{\prime} s^{\prime} i l s$ sont éléments addibles de $I^{\prime}$ un des $\bar{A}^{-\xi}, e$. Le milieu de $L^{\prime}$ sera $G^{\prime}=\underset{e \geq 1}{U} G_{e}$. On est ainsi ramené à l'étude des agglutinés $\bar{A}^{\xi}, e$, d'où les résultats annoncés grâce aux lemmes 3 et 4 .

\section{Remarques complémentaires sur l'agglutination par un grade, ou} par un ensemble de grades.

$\left.3^{\circ}\right)$ Soient $D$ une partie non vide de $\Delta$, et $s$ un élément donné de $D$. Nous allons définir un $A$-monel agglutiné $\bar{A}(s, D)$ par une légère extension de la définition jointe au lemme 3 . Soit a $\in A . . \Omega$; si, pour toutes les factorisations possibles $a=f_{1} \ldots \ldots f_{q}$ (les $f_{j} \in A$ ) et $s=d_{1} \ldots d_{r}$ (les $d_{i} \in D$ ), le produit $\omega\left(f_{1}\right) \ldots \omega\left(f_{q}\right) d_{1} \ldots d_{r}$ est associatif et non nul, sa valeur $\omega(a) s$ est par définition le grade $\omega^{\prime}(a)$ de a dans $\bar{A}(s, D) \cdot$; dans les autres cas, ce grade est nul.

Par une démonstration presqu'identique à celle du leme 3 , on peut montrer que, pour $c$ et $a \in A$, la relation $\omega^{\prime}(c$ a) $\neq 0$ implique $\omega^{\prime}(c \quad a)=\omega(c) \omega^{\prime}(a)$ et que $\bar{A}(s, D)$ est un A-monel intermédiaire entre $A$ et $\bar{A}$. $\mathrm{Si}$, de plus, tout élément $\mathrm{d}$ de $\mathrm{D}$ associe dans $\Delta, \bar{A}(s, D)$ est un anel. Si l'on prend $s=\xi^{e}$ et $D=\left\{\xi, \xi^{2}, \ldots, \xi^{e}\right\}$, on obtient comme cas particulier $\bar{A}^{\xi}, e$. 
Lorsque $D \subset D^{\prime} \subset \Delta$, le $A-$ monel $\bar{A}\left(s, D^{\prime}\right)$ est un agglutiné de $\bar{A}(s, D)$. En effet, des factorisations plus nombreuses dans $D^{\prime}$ que dans $D$ pour $s$, peuvent augmenter les cas de non-associativité, donc augmenter le milieu.

$\left.4^{\circ}\right)$ Soient $S \subset D$ deux parties non vides de $\Delta$, s étant multiplicativement stable (les deux principaux cas à envisager sont $D=S$ et $D=\Delta)$. La réunion $R=\bar{A}(S, D)=\bigcup_{S \in S} \bar{A}(S, D)$ peut être munie d'une structure de A-monel. D'abord, $R$ est stable par multiplication par les éléments de $\Lambda$. Si $\alpha=\sum_{i} a_{i} \in \bar{A}(s, D)$, il est clair que $\alpha \in \bar{A}(r s, D)$, pour tout $r \in S$. Par définition, les éléments $\alpha$, et $\beta=\sum_{j} b_{i}$ de $R$ seront addibles dans $R$, s'il existe $t$ tels que les $\omega_{t}^{\prime}\left(a_{i}\right)$ et $\omega_{t}^{\prime}\left(b_{j}\right)$ non nuls soient égaux ( $\omega_{t}^{\prime}$ désignant le grade dans $\bar{A}(t, D)$ ), c'est-à-dire s'ils sont éléments de $\bar{A}(t, D)$, et $y$ sont addibles. On voit alors que $R$ est un A-monel dont le milieu est $\mathcal{O}=\bigcup_{s \in S} \mathcal{O}_{s}$, où $\mathcal{G}_{s}$ est le milieu de $\bar{A}(s, D)$. En effet, si $\alpha=\sum_{i} a_{i}$ est tel que (dans $\bar{A}(s, D)$ ) $w_{s}^{\prime}\left(a_{i}\right)=0$ pour tout $i$, et si $\beta=\sum_{j} b_{j} \in \bar{A}(r, D)$, il est clair que $\omega_{r s}^{\prime}\left(a_{.}\right)=0$, que $\beta \in \bar{A}(r s, D)$, donc que $\alpha$ et $\beta$ sont addibles (axiome $B_{3}$ ). D'autre part, soient $\alpha, \beta, \gamma \in \bar{A}(S, D)$, $\alpha$ et $\beta$ étant éléments addibles de $\bar{A}(r, D), \beta$ er $\gamma$ éléments addibles de $\bar{A}(s, D)$, et $\beta \notin G$. Donc, $\alpha$ et $\beta$ (resp. $\beta$ et $\gamma$ ) sont éléments addibles de $\bar{A}(r s, D)$, et $\varepsilon \notin \mathrm{O}_{\mathrm{rs}}$. Alors, $\alpha$ et $\gamma$ sont addibles dans $T$ ( $\left.r \mathrm{~s}, \mathrm{D}\right)$, donc dans $\mathrm{R}$ (axiome $\mathrm{B}_{4}$ ). La vérification pour $\mathrm{R}$ des autres axiomes est triviale.

\section{$\S 4$ - DEFINITION DES POLYNOMES.}

Nous supposerons toujours commutatifs les anels et annérdes considérés, ainsi que leurs graduations éventuelles. Soit $\mathrm{A}$ un anel donné, de graduation donnée $\Delta$. Rappelons que $\omega(a)$ désigne le grade d'un élément a $\in A$, avec $\omega(\mathrm{a})=0$ si a est dans le milieu. On note $\omega(\mathrm{A})$ l'ensemble des grades des éléments de $\mathbf{A}$ (c'est un support de la graduation propre $\Delta \circ), \bar{A} I^{\prime}$ anneau de $A$, et $\Delta^{*}=\Delta . .\{0\}$. Lorsque $A$ est unitaire, $\omega(1)$ peut ou non etre unité dans $\Delta$. On appellera prolongement de la graduation $\Delta$ de $A$ toute graduation $\Delta^{\prime}$ de $A$ vérifiant les conditions suivantes : il existe une surjection $\theta: \Delta^{\prime} \rightarrow \Delta$, injective sur $I^{\prime}$ image réciproque $\theta^{-1}\left(\Delta^{*}\right)$; on a $\theta(0)=0$; quels que soient $d^{\prime}$ et $d_{1}^{\prime} \in \theta^{-1}\left(\Delta^{*}\right)$, on $a \theta\left(d^{\prime}\right) \theta\left(d_{1}^{\prime}\right)=\theta\left(d^{\prime} d_{1}^{\prime}\right)$ chaque fois que le premier membre est non nul. Lorsque cette dernière condition est imposée même quand le premier membre est nul, on dira que $\Delta^{\prime}$ 
prolonge strictement $\Delta ;$ dans ce cas, $\Delta$ peut être identifié à la partie $\theta^{-1}\left(\Delta^{*}\right) \cup\{0\}$ de $\Delta^{\prime}$. Par exemple, la graduation $\Delta$ de $A$ est un prolongement, en géneral non strict, de la graduation propre $\Delta \circ$.

Soient $p$ indéterminées $x_{1}, \ldots \ldots, x_{p}$. Pour toute suite $(i)=\left(i_{1}, \ldots, i_{p}\right)$ de $p$ entiers naturels, on pose $i=i_{1}+\ldots+i_{p}$. Soit la somme formelle "a p variables" de monômes :

$$
\begin{aligned}
f=f(x)=f\left(x_{1}, \ldots, x_{p}\right) & =\sum_{0 \leqslant n}\left(a(i), 1+\ldots .+a_{(i), r_{i}}\right) x_{1}^{i} \ldots \ldots x_{p}^{i} p \\
& =\sum_{0 \leqslant i \leqslant n} \alpha_{(i)} x^{(i)}, \text { avec les cofacteurs } a_{(i), s} \in A,
\end{aligned}
$$

deux termes d'une même parenthèse $n$ 'étant pas addibles. Une telle somme formelle est simplement un polynome à $\mathrm{p}$ variables sur l'anneau $\overline{\mathrm{A}}$, les coefficients $\alpha_{(i)} \in \overline{\mathrm{A}}$ étant réduits. Elle est dite nulle si tous ses coefficients sont nuls. Deux sommes sont égales si elles sont formées des mêmes monômes ( $c^{\prime}$ est-à-dire si les coefficients correspondants sont égaux).

Définition 1: On se donne un anel $A$, sa graduation $\Delta$, et un p-uple $\xi=\overline{\left(\xi_{1}, \ldots, \xi_{p}\right)} \in \Delta^{p}$. La somme formelle $f$ sera dite un polynôme à $p$ variables sur $A$, de pente $\xi$ (ou, plus brievement un polynôme de pente $\xi$, ou un 5 -polynome, s'il n'y a pas d'ambigurté) si et seulement si, quel que soit le sur-anel $B$ de $A$, de graduation $\Delta$, et quel que soit $x=\left(x_{1}, \ldots, x_{p}\right) \in B^{P}$ avec, pour tout $j=1, \ldots, p, \omega\left(x_{j}\right)=\xi_{j}, f(x)=f\left(x_{1}, \ldots, x_{p}\right)$ a tous ses termes addibles. $\xi_{j}$ est appelé le grade de la variable $x_{j}$, et $I$ 'on note $w\left(x_{j}\right)=\xi_{j}$.

Lorsqu' on suppose $A$ unitaire et $\omega(1)$ unité de $\Delta$, on exige que 1 soit aussi unité de $B$.

Définition 2: Supposons donnés un anel A, et une graduation $\Delta$ de $A$. Une somme formelle $f$ sera dite un pré-polynôme si et seulement si il existe un prolongement $\Delta^{\prime}$ de $\Delta$ et un p-uple $\xi \in \Delta^{\prime P}$ tels que $f$ soit un క-polynôme.

Par suite, deux polynômes $f$ et $g$ seront considérés comme distincts, ou bien si les sommes formelles $f$ et $g$ sont distinctes, ou bien si elles sont égales, mais si on les considère comme polynômes avec des pentes différentes. La définition 2 conduit à étudier si une somme formelle donnée est ou non un pré-polynôme. Ce problème, que l'on traitera ailleurs, a été résolu par M. Krasner [4] dans le cas des corpordes "sans torsion" (c'est-à-dire tels que $\Delta_{0}^{*}$ soit un groupe sans torsion). 
A, $\Delta$ et $\xi \in \Delta^{\mathrm{P}}$ étant donnés, $f$ est un $\xi$-polynôme si et seulement si, quels que soient $B$ et $x$ envisagés à la définition 1 , les grades $\omega\left(a(i), s{ }^{(i)}\right)$ non nuls sont égaux. Si, dans $\Delta_{1}$ trois grades $\omega_{1}, \omega_{2}, \omega_{3}$ ont un produit non associatif, quel que soit le sur-anel $B$ de $A$, et quels que soient $b_{1}, b_{2}$ et $b_{3} \in B$ de grades respectifs $\omega_{1}, \omega_{2}, \omega_{3}$, le produit $b_{1} b_{2} b_{3}$ appartient au milieu de $B$. Cette remarque conduit à la définition suivante du $\xi$-grade, noté $\omega^{\prime}\left(a x^{(i)}\right)$, d'un monôme $a x^{(i)}=a x_{1}^{i_{1}} \ldots . x_{p}{ }^{i}$ (avec $a \in A$ ). On note 尺 le milieu de $A$.

Définition 3 : A, $\Delta$ et $\xi \in \Delta^{\mathrm{P}}$ sont donnés. Si a $\notin \Omega$, et si, pour toute factorisation $a=a_{1} \ldots a_{q}$ (les $a_{j} \in A$ ) de $a$, le produit $\omega\left(a_{1}\right) \ldots \omega\left(a_{q}\right) \xi_{1} \ldots \xi_{1} \xi_{2} \ldots \xi_{p} \ldots \xi_{p}$ est associatif et non nul (le facteur $\xi_{k}$ figurant $i_{k}$ fois), sa valeur $\omega(a) \xi^{(i)}$ est le $\xi$-grade du monome a $x^{(i)}$. pans tous les autres cas, ce $\xi$-grade est nul. (On dit grade, s'il n'y a pas d'ambiguité sur $\xi$ ).

Pour la commodité, on notera le produit précédent plus brièvement par $\omega\left(a_{1}\right) \ldots \omega\left(a_{q}\right) \xi_{1}^{i} \ldots \xi_{p}^{p}$, grace à un abus d'écriture. Précisons les divers cas ou $\omega^{\prime}\left(a x^{(i)}\right)=0$ : Ou bien $a \in \Omega$. Ou bien a $\notin \Omega$, et pour toute factorisation de $a$, le produit $\omega\left(a_{1}\right) \ldots \omega\left(a_{q}\right) \xi_{1}{ }_{1} \ldots \xi_{p}^{i_{p}}$ est associatif et nul (outre cette condition d'associativité, il suffit qu'il soit nul pour une factorisation de a). Ou bien a $\notin \Omega$, et il existe une factorisation $a=a_{1} \ldots a_{q}$ telle que $\omega\left(a_{1}\right) \ldots . \omega\left(a_{q}\right) \xi_{1}^{i_{1}} \ldots \xi_{p}^{i_{p}}$ ne soit pas associatif. Les monomes de grade nul ne prennent que des valeurs appartenant au milieu de B. Donc :

Lemme 1 : Pour que $f$ soit un polynôme de pente $\xi$, il suffit que ses monômes de grade non nul aient le même grade.

Ce grade commun est appelé le $\xi$-grade ou la $\xi$-hauteur de $f$ (on dit pente et hauteur par analogie avec la théorie des corpordes de M. Krasner [4]). On note $\omega^{\prime}(f(\xi))$, ou $\omega^{\prime}(f) s^{\prime} i l$ n'y a pas d'ambigurté sur la pente. On pose $\omega^{\prime}(f)=0$ lorsque tout monôme est de grade nul. Il est clair que le produit de deux monômes, dont un est de grade nul, a un grade nul. Si le monôme $(a b) x^{(i)+(j)}$ vérifie la condition d'associativité de la définition 3, et est de grade non nul, il en est évidemment de même des monômes facteurs a $x^{(i)}$ et $b x^{(j)}$, et le grade du produit $(a b) x^{(i)+(j)}$ est bien sur égal au produit des grades de a $x^{(i)}$ et $b x^{(j)}$. Ces remarques entrainent le résultat : 
Lemme 2: Si deux $\xi$-polynômes $f$ et $g$ vérifient la condition suffisante du lemme 1 , f g la vérifie aussi, dnnc est un $\xi$-polynôme. De plus, si $\omega^{\prime}(f g) \neq 0$, on a $\omega^{\prime}(f g)=\omega^{\prime}(f) \omega^{\prime}(g)$.

En effet, dans le produit formel $f \mathrm{~g}$ non réduit, les monômes auront pour grade ou 0 , ou $\omega^{\prime}(f \mathrm{~g})$. Il en est de même après réduction.

Notons $A_{\xi}[X]=A_{\left(\xi_{1}, \ldots, \xi_{p}\right)}\left[X_{1}, \ldots, X_{p}\right]$ I'ensemble des polynomes de pente $\xi$ vérifiant la condition suffisante du lemme 1 , et montrons qu'il a une structure d'anel. Deux éléments $f$ et $g$ seront dits addibles si les grades $\omega^{\prime}(f)$ et $\omega^{\prime}(g)$ sont égaux, ou si l'un de ces grades est nul. Alors, la somme formelle $f+g$ est dans $A_{\xi}[X]$ et sera leur somme. $\boldsymbol{O}=\left\{\varphi \in A_{\xi}[X] ; \omega^{\prime}(\varphi)=0\right\}$ sera le milieu de $A_{\xi}[X]$, qui satisfait évidemment aux axiomes des anels. En particulier, le lemme 2 montre que $A_{\xi}[\mathrm{X}]$ est une partie multiplicativement stable de $\bar{A}[\mathrm{X}]$, et que les axiomes $D_{1}$ et $D_{5}$ sont vérifiés. Ce lemme montre enfin que $\Delta$ est une graduation du sur-anel $A_{\xi}[\mathrm{X}]$ de A (sur-anel unitaire si A est unitaire).

Théorème : Soient $A$ un anel de graduation $\Delta$, et un $p$-uple $\xi \in \Delta^{p}$. L'ensemble des polynômes à $p$ variables sur $A$ et de pente $\xi$, est l'anel $A_{\varepsilon}[\mathrm{X}]$ des sommes formelles dont les monomes de $\xi$-grade non nul ont même $\xi$-gradé. $\Delta$ est une graduation de ce sur-anel de A. Si de plus, A est unitaire, et si $w(1)$ est unité dans $\Delta, A_{\xi}[\mathrm{X}]$ est sur-anel unitaire de $A$, et sa graduation propre contient les $\xi_{j}$.

Lorsque A est unitaire et que $\omega(1)$ est unité de $\Delta$. considérons un polynôme $f$ de pente $\xi$ (c'est-à-dire vérifiant seulement les conditions de la définition 1). En Darticulier, si on prend $B=A_{\xi}[X]$ et $x=X$, le grade de $x_{j}=x_{j}$ est $\omega^{\prime}\left(x_{j}\right)=\omega(1) \omega\left(x_{j}\right)=\omega(1) \xi_{j}=\xi_{j}$. Alors, $f(x)=f(x)$ doit nécessairement avoir ses termes addiblec dans $A_{\xi}[X]$, donc ses monômes de grade non nul ont le même grade.

Lorsque $A$ n'est pas unitaire (attention : $x_{j} \notin A_{\xi}[X]$ ), construisons un sur-anel $B^{\prime}$ de $A_{g}[X]$ cornme suit. Dans la nouvelle somme formelle $F=\sum_{0 \leqslant i \leqslant n}\left(\alpha_{(i)}+n_{(i)}\right) x^{(i)}$ où $\alpha_{(i)} \in \bar{A}$ et $n_{(i)} \in \mathbb{Z}$, le క̈-grade d'un monôme sera défini pour les uns par la définition 3 . Pour un monôme $n x^{(i)}$ ( $n$ entier $\neq 0$ ), ce $\xi$-grade sera $\xi_{1}^{i_{1}} \ldots \xi_{\mathrm{p}}^{i_{p}}$ si ce produit est assnciatif et non nul, et 0 dans le cas contraire. Notons $B^{\prime} I^{\prime}$ ensemble des sommes formelles $F$ dont les monômes de $\boldsymbol{\xi}$-grade non nul ont même grade ; ce grade est noté $\omega^{\prime}(F)$ et appele la $\xi$-hauteur ou le $\xi$-grade de $F$ (avec $\omega^{\prime}(F)=0$ si tout monôme a un grade nul). Comme pour $A_{\xi}[X]$, en considérant d'abord le produit 
de dcux monômes, on voit que le lemme 2 est encore vrai dans $B^{\prime}$. Comme pour $A_{\xi}[X]$, on en déduit que $B^{\prime}$ est un sur-anel de $A$ de graduation $\Delta ;$ il est clair que $B^{\prime}$ est un sur-anel de $A_{\xi}[\mathrm{X}]$. De plus, $1 \mathrm{X}_{\mathrm{j}}$ (avec $1 \in \mathbb{Z}$ ) est un élément de $B^{\prime}$ dont le $\xi$-grade est $\omega^{\prime}\left(1 x_{j}\right)=\xi_{j}$ par définition. Alors, soit $f$

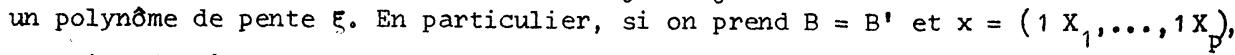
on voit, de même que ci-dessus, que les monômes de grade non nul de $f$ ont le mêne grade. Le lemme 1 complète la démonstration dans les deux cas.

Remarquons que, même si $\mathrm{A}$ est un annéîde, $\mathrm{I}$ 'anel $\mathrm{A}_{\xi}[\mathrm{X}] \mathrm{n}$ 'est pas forcément un annéride, puisque des monômes non nuls peuvent avoir un grade nul (définition 3 ).

Corollaire : Soient $\mathrm{A}$ un anel de graduation $\Delta, \xi$ et $\eta \in \Delta$, et $\mathrm{X}$ et $\mathrm{Y}$ deux indéterminées. $\mathrm{A}_{\{\xi, \eta\}}[\mathrm{X}, \mathrm{Y}]$ et $\left(\mathrm{A}_{\xi}[\mathrm{X}]\right)_{\eta}[\mathrm{Y}]$ représentent le même anel.

Soit un polynôme $\left.f(X, Y) \in A_{\{\xi}, \eta\right\}[X, Y]$, donc dont les monômes a $\mathrm{X}^{i} \mathrm{Y}^{j}$ de grade non nul ont même grade. I]. peut être considéré comme une somme formelle polynomiale en $Y$, à cofacteurs dans $A_{\xi}[X]$; de plus, la condition vérifiée par les grades des monômes a $\mathrm{X}^{i} \mathrm{Y}^{j}$ entrâne que cette somrne est un polynome en $Y$.

Inversement, un polynome $g(Y) \in\left(A_{\xi}[X]\right) \eta^{[Y]}$ a pour cofacteurs des polynômes $\varphi(X)$ éléments de ${ }^{A}[X]$. Chaque fois qu'à un de ces cofacteurs $\varphi(x)$ correspond un "monôme" $\varphi(X) Y^{j}$ de grade non nul $\sigma(g)$, le même "monôme" peut être considéré comme une somme formelle appartenant à $\bar{A}[\mathrm{X}, \mathrm{Y}]$, mais dont les monômes sont, ou de grade nul, ou de grade égal à $\sigma(g)$, grâce à la définition 3 . Donc $g$ est élément de ${ }^{A}\{\xi, \eta \eta\}[X, Y]$. Ceci étant, l'identité des structures d'anel est évidente. Ce corollaire s'étend à $p$ variables $X_{1}, \ldots, X_{p}$ et $q$ variables $Y_{1}, \ldots, Y_{q}$.

Substitutions dans un polynôme. Soient A un anel, $\Delta$ une graduation

de $A, p$ et $q$ deux entiers $\geq 1, \xi \in \Delta^{p}$ et $\eta \in \Delta^{q}$. Soit $f$ un polynôme sur $A$, à $p$ variables $x_{j}(j=1, \ldots, p)$, de pente $\xi$, et soient $g_{1}, \ldots, g_{p}$ des polynomes sur A à $q$ variables $Y_{k}(k=1, \ldots, q)$, chacun de pente $\eta$. Si, quel que soit $j=1, \ldots, p_{1}$ le $\eta$-grade du polynome $g$ est $\xi_{j}$ ou 0 , il est clair que la somme formelle $h=f\left[g_{1}(Y), \ldots, g_{P}(Y)\right] \in \bar{A}\left[Y_{1}, \ldots, Y_{q}\right]$ est un polynome de pente $\eta_{0}$ En effet, puisque $f$ est un $\xi$-polynôme, donc vérifie les conditions du théorème, il suffit d'utiliser le lemme 2 pour étudier le n-grade des monômes produits dans l'expression non réduite de $h$. On dit que $\underline{h}$ est obtenu par substitution, dans $f$, des polynômes $g$ aux variables $X_{j}$. 
Cas d'un annérde : Soient $\mathrm{A}$ un annérde de graduation $\Delta$, et $\xi \in \Delta$. Notons $\Gamma=[\omega(\mathrm{A}), \xi]$ la partie de $\Delta$ engendrée par $\omega(\mathrm{A})$ et $\xi$. Pour que $\mathrm{A}_{\xi}[\mathrm{X}]$ soit un annélde, il faut et il suffit que $\xi$ associe extérieurement dans $\Gamma$ et que $\xi$ ne divise pas 0 dans $\Gamma$. En effet, la condition est évidemment nécessaire. Inversement, si elle est vérifiée, on sait (II, 3, démonstration du lemme 4) que $\xi^{i}$ associe dans $\Gamma$. Le seul monôme a $x^{i}$ de grade $\omega^{\prime}\left(a x^{i}\right)$ nul, est celui où $a=0$. Si $a \neq 0, I^{\prime}$ associativité montre que $\omega^{\prime}\left(a x^{i}\right)=\omega(a) \xi^{i}$, qui est non nul puisque $\xi$ ne divise pas zéro.

Soit $f$ un polynome à $p$ variables sur un anneau quasi-gradué $\bar{A}$, de graduation $\Delta_{\text {. }} \mathrm{S}^{\prime}$ il existe un prolongement $\Delta^{\prime}$ de la graduation $\Delta$ de $\bar{A}_{\text {, }}$ et un p-uple $\xi=\left(\xi_{1}, \ldots, \xi_{p}\right) \in \Delta^{\wedge P}$ tels que, quel que soit le sur-anneau quasigradué $\bar{B}$ de $\bar{A}$, de graduation $\Delta^{\prime}$, et quelles que soient les valeurs $x_{j} \in \bar{B}$, homogènes et de grade $\xi_{j}$ respectivement, données aux variables $x_{j}$. la valeur $f\left(x_{1}, \ldots, x_{p}\right)$ prise par $f$ est homogene, alors on dit que $f$ est un polynome homogène pour les grades $\xi_{j}$ attribués aux variables (ou pour la pondération $\xi_{j}$ des variables $x_{j}$ ). Le théorème précédent montre que, pour $f$, etre ou non homogène ( $\Delta^{\prime}$ et $\xi$ étant fixés) pour les variables $x_{j}$ pondérées par les grades respectifs $\xi_{j}$, dépend uniquement de $\Delta^{\prime}$ et de $\xi$, sans que les sur-anneaux $\bar{B}$ aient à intervenir. 
On rappelle que les anels considérés sont toujours commutatifs, ainsi que leurs graduations éventuelles. Dans ce chapitre, bien des résultats seront donnés sans démonstration, car les preuves correspondantes en sont aisées, sinon triviales. Rappelons (II, $\S 2$, th. 1) la loi modulaire propre aux anels et monels, qui se réduit à la forme classique pour les annérdes et modulordes.

\section{$\$ 1$ - PROPRIETES DES HOMOMORPHISMES.}

Lemme 1 : Soient $M$ et $M_{1}$ deux ensembles, $G$ et $\mathrm{I}$ deux parties de $M_{\text {, }}$ $O_{1}$ une partie de $M_{1}$. Soit $u: M \rightarrow M_{1}$ une application telle que $u(O) \subset O_{1}$. Les conditions suivantes sont équivalentes : (1) $u(L . . L \cap O) \cap\left\{G_{1} \ldots u(L \cap \mathcal{L})\right\}=\emptyset$; (2) $u(L) \cap O_{1} \subset u(L \cap O)$.

Si (2) est vérifiée, soit y $\in u(L . . L \cap G) \cap\left\{O_{1} \ldots u(L \cap G)\right\}$; on déduit y $\in u(L) \cap G_{-1}=u(L \cap G)$, ce qui contredit $y \in G_{1} \ldots u(L \cap O)$, puisque $u(0) \subset G_{1}$; on a donc (1). Inversement, si (1) est vraie, prenons y $\in u(L) \cap G_{1}$. Supposons que $y \in u(L \cap O)$; comme $y \in u(L)$, on a $y \in u(L \ldots L \cap O)$, et comme y $\in \mathcal{Q}_{1}$, on a y $\in \mathcal{O}_{1} \ldots u(L \cap \mathcal{O})$; on en déduit (par (1)) que y $\in \emptyset$. Donc $u(L) \cap \mathcal{O}_{1} \subset \mathrm{u}(\mathrm{I} \cap \mathcal{O})$. (L'inclusion inverse est triviale)。

Définition 1 : Soient $M$ et $M_{1}$ deux A-monels, $G$ et $O_{1}$ leurs milieux respectifs, et $u: M \rightarrow M_{1}$ un quasi-morphisme. Pour un sous-monel $L$ de $M$, on dira que u vérifie $[m, L], s^{\prime} i l$ vérifie les conditions équivalentes du lemme. On dira que u vérifie [m], s'il vérifie [m,L] pour tout sous-monel $L$ de M. On notera de même les propriétés analogues pour un quasi-morphisme d'anels, en remplaçant $M, M_{1}, O, O_{1}$ respectivement par des anels $A_{,} A_{1}$, leurs milieux $\Omega, \Omega_{1}$, et en remplaçant les sous-monels $\mathrm{L}$ par les idéaux $\mathfrak{J}$ de A.

Remarque 1 : Dans le cas des annérdes et des modulordes, les conditions précédentes sont trivialement vérifiées.

Remarque 2 : Lorsque $\mathrm{L} \subset \mathcal{O}, \mathrm{u}$ vérifie $[\mathrm{m}, \mathrm{L}]$.

Remarque 3 : La condition $[\mathrm{m}, \mathrm{M}]$ équivaut à $u(\mathbf{O}) \supset \mathbf{O}$, lorsque $u$ est surjectif, et a $\bar{u}^{-1}\left(\mathcal{O}_{1}\right) \subset \bigcup \bigcup$ lorsque $u$ est injectif. On a le résultat analogue pour les anels.

La condition $[m, M]$ s'écrit $u(M \circ . \mathcal{O}) \cap\left\{\mathcal{O}_{1} \circ u(\mathcal{O})\right\}=\emptyset$ ou $u(M) \cap G_{1}=u(\mathcal{O})$. La première assertion est triviale. Pour le cas injectif, on a $\vec{u}^{-1}\left(\mathcal{O}_{1}\right)=\left\{x \in M ; u(x) \in \mathcal{O}_{1}\right\}$, et par injectivité $\vec{u}^{-1}\left(\boldsymbol{O}_{1}\right)=\boldsymbol{O} \cap \mathrm{s}$ 
avec $S=\left\{x \in M . . \mathcal{O} ; u(x) \in \mathcal{O}_{1} \ldots u(\mathcal{O})\right\}$. Ceci montre l'équivalence de $\bar{u}^{-1}(\mathcal{O})=\mathcal{O}$ avec $s=\emptyset$, c'est-à-dire avec $[\mathrm{m}, \mathrm{M}]$.

Remarque 4 : Si $K$ est un sous-monel de $M$, I'homomorphisme canonique $\varphi: M \rightarrow M / K$ vérifie $[m, M]$ par définition de $M / K$, et, pour la même raison, vérifie $[\mathrm{m}, L]$ pour tout sous-monel $L$ contenant $K$. Mais $\varphi$ ne vérifie pas forcément $[\mathrm{m}, \mathrm{L}]$ lorsque $\mathrm{L}$ et $\mathrm{K}$ ne sont pas comparables. De même, si $\Im$ est un idéal d'un anel A, I'homomorphisme $\varphi: A \rightarrow A / \Im$ vérifie $[m, a]$ pour tout idéal a contenant $\mathfrak{3}$.

Exemple contraire 1 : Soit $\mathbb{Z}$ l'anneau des entiers. Considérons le $\mathbb{Z}$-monel $M=M_{a} \cup M_{b}$, or $M_{a}$ et $M_{b}$ sont isomorphes au $\mathbb{Z}$-module $\mathbb{Z} \times \mathbb{Z}$, et ont en commun le milieu $\mathbf{O}=\mathbb{Z}(1,1)$. Prenons les sous-monels $\mathrm{K}=\mathrm{K}_{\mathrm{a}} \cup \mathrm{K}_{\mathrm{b}}=\mathbb{Z} \times(0) \cup \mathbb{Z} \times(0)$ et $\mathrm{L}=(0) \times \mathbb{Z} U(0) \times \mathbb{Z}$ dont la somme $\mathrm{K}+\mathrm{L}$ est $M$. Notons $\varphi: M \rightarrow M / K$. Tout élément $(m, n)_{a} \in M_{a}$ est congru modulo $K$ à $(n, n) \in \mathcal{O}$. Donc $M / K$ est son propre milieu $\mathcal{O}^{\prime}$. Tout élément de $M / K$, soit $(n, n)+K$, est image par $\varphi$ de $(0, n)_{a} \in L$. Donc $\varphi(L)=M / p$, et on a $\varphi(L) \cap \boldsymbol{O}^{\prime}=\mathrm{M} / \mathrm{P}$. Par contre, on a $\mathrm{L} \cap \mathcal{O}=(0,0)$ dont $I^{\prime}$ image est le zéro de $M / P$. Donc $\varphi$ ne vérifie pas $[\mathrm{m}, \mathrm{L}]$.

Théorème 1 : Soient $M$ et $M_{1}$ deux $A$-monels de milieux respectifs $\mathcal{O}$ et $\mathcal{O}_{1}$, et soit $u: M \rightarrow M_{1}$ un quasi-morphisme de A-monels. 1 - Pour tout sous-monel $L_{1}$ de $M_{1}, u^{-1}\left(L_{1}\right)$ est sous-monel de $M_{0}$. En particulier, le noyau Ker $u=u^{-1}(0)$ de $u$ est un sous-monel. 2 - Soient $B$ une partie de $A$, et $X$ une partie de $M$; on a $u(B X) \subset B u(X)$. On a l'égalité si de plus $u$ est un homomorphisme vérifiant $\bar{u}^{-1}\left(O_{1}\right) \subset \boldsymbol{O}$.

3 - Soit $L$ un sous-monel de $M$. Si u est un homomorphisme vérifiant $[m, L]$, $u(L)$ est un sous-monel de $M_{1}$.

4 - Si $u$ est un homomorphisme vérifiant $[m, M]$, pour tout sous-monel $L$ contenant Ker $u, u(L)$ est un sous-monel.

Remarque 5 : Pour les moduloides, les conditions $[\mathrm{m}, \mathrm{L}]$ et (dans 2) $\bar{u}^{-1}\left(\mathbf{G}_{1}\right) \subset \mathcal{O}$ sont triviales. En particulier, l'image d'un modulorde par un homomorphisme est un moduloide.

1 et 2 se montrent sans difficulté (pour 2, on utilise la notion de combinaison linéaire donnée en II, $\S 2$ b). Pour 3 et 4 , on a $u(0)=0$, et pour tout $a \in A$ et tout $x \in L$, on $a u(a x)=a u(x)$. Soient $x^{\prime}=u(x)$ et $y^{\prime}=u(y)$ deux éléments addibles de $u(L)$, avec $x, y \in L$. Lorsque $x^{\prime}$ et $y^{\prime} \notin \mathcal{O}_{1}$, u étant un homomorphisme, $x$ et y sont addibles,

$d^{\prime}$ où $x^{\prime}-y^{\prime}=u(x)-u(y)=u(x-y) \in u(L)$. Lorsque $x^{\prime} \in O_{1}$, on a $x^{\prime} \in u(L) \cap O_{1}$. Dans le cas 3 , la condition $[m, L]$ nontre que $x^{\prime} \in u(L \cap O)$ et 
il existe $1 \in L \cap \mathcal{L}$ tel que $u(1)=x^{\prime}$. Comme 1 \#y, on a $x^{\prime}-y^{\prime}=u(l)-u(y)=u(1-y) \in u(L)$. Dans 1 e cas 4 , on a $x^{\prime} \in u(M) \cap \mathcal{O}_{1}=u(M \cap G)=u(\mathcal{O})$, et il existe $z \in \mathcal{G}$ tel que $u(z)=x^{\prime}$. Mais on $a u(x-z)=u(x)-u(z)=0$, d'où $x-z \in \operatorname{Ker} u \subset L$, et enfin $z \in L$, donc $z \in I \cap \mathcal{O}$. Alors, y et $z$ étant des éléments addibles de $L$, on a $x^{\prime}-y^{\prime}=u(z)-u(y)=u(z-y) \in u(L)$.

Lemme?: Soit $u: M \rightarrow M_{1}$ un quasi-morphisme de A-monels.

(1) Si $u$ est injectif, Ker $u=(0)$.

(2) Si $u$ est un homomorphisme vérifiant $[m, M]$, et si $K \in r u=(0)$, alors $u$ est injectif.

(1) est trivial. Soient $x, y \in M$ tels que $u(x)=u(y)$. ou bien $u(x) \notin \mathcal{O}_{1}$, et $u$ étant un homoinorphisme, on a $x \not y$, d'où on déduit $0=u(x)-u(y)=u(x-y)$ et $x-y=0$. ou bien $u(x) \in G_{1}$, et grace à $[m, M]$, on a $u(x) \in u(M) \cap \mathcal{O}_{1} \subset u(0)$. Donc il existe $z \in \mathcal{O}$ tel que $u(x)=u(z)$. Mais $z$ \#x, et on déduit comme plus haut $x=z$. Il en est de même pour $y$, et on $a x=y$.

Lemme 3 : Soient $M$ et $M_{1}$ deux A-monels, de milieux respectifs $\mathcal{O}$ et $\mathcal{O}_{1}$. Soit $u: M \rightarrow M_{1}$ un homomorphisme bijectif. Les conditions suivantes sont équivalentes :

(1) $u$ est un isomorphisme (

(2) $\bar{u}^{-1}$ est un homomorphisme.

(3) u vérifie $[\mathrm{m}, \mathrm{M}]$.

(4) $\bar{u}^{-1}\left(\mathcal{O}_{1}\right) \subset \mathcal{O}$.

Il reste à montrer l'équivalence de (1) et (2), ce qui est facile. Dans le cas des moduloides, ces conditions sont trivialement vérifiées, de même que celles de la définition suivante.

Définition 2 : Soient $M$ et $M_{1}$ deux A-monels, de milieux respectifs Oet $O_{1}$. Un homomorphisme injectif $u: M \rightarrow M_{1}$ est dit un monomorphisme s'il vérifie les conditions équivalentes suivantes :

(1) La restriction $v: M \rightarrow u(M)$ de $u$ est un isomorphisme.

(2) $u(M) \cap \mathbf{O}_{1} \subset u(\mathcal{O})$.

(3) $u$ vérifie $[\mathrm{m}, \mathrm{M}]$.

(4) $\vec{u}^{-1}\left(O_{1}\right) \subset \mathcal{O}$.

Si (1) est vérifiée, $u(M) \cap O_{1}$ est le milieu du sous-monel $u(M)$, et $v$ vérifie $[m, M]$, donc $u$ aussi, $\operatorname{car} v(M) \cap\left[u(M) \cap O_{1}\right] \subset v(M \cap O)$ s'écrit $u(M) \cap O_{1} \subset u(\mathcal{O})$. Inversement, si $u$ vérifie $\left[m, M_{1}\right], u(M)$ est un sous-monel. On a enfin $\vec{v}^{-1}\left(\mathcal{O}_{1} \cap u(M)\right)=\vec{u}^{-1}\left(\mathcal{O}_{1} \cap u(M)\right)=\vec{u}^{-1}\left(\mathcal{O}_{1}\right) \subset \mathcal{O}$, et $v$ est un isomorphisme. Le reste est connu. 
Lemme 4 : Un homomorphisme $u: M \rightarrow M_{1}$, de noyau nul et vérifiant $\mid[m, M]$, est un monomorphisme.

En effet, il est injectif (lemme 2).

Théorème 2 : 1 - Soient $M$ et $M_{1}$ deux A-monels, $P$ un sous-monel de $M$ et $\varphi$ la surjection $M \rightarrow M / P$. Si $u: M \rightarrow M_{1}$ est un quasi-morphisme tel que $P \subset$ Ker $u$, il existe un quasi-morphisme $u: M / P \rightarrow M_{1}$ tel que $u=\bar{u} \circ \varphi$. Si $u$ est un homomorphisme, $\bar{u}$ aussi.

2 - Si, de plus, le quasi-morphisme $u$ vérifie [m,L] pour un sous-monel L contenant $P$, alors $\bar{u}$ vérifie $\left[m, L^{\prime}\right]$ où $L^{\prime}$ est le sous-monel $\varphi(L)$. Si, de plus, $u$ vérifie $[\mathrm{m}, \mathrm{L}]$ pour tout sous-monel $\mathrm{L}$ contenant $\mathrm{P}$, $\bar{u}$ vérifie $[\mathrm{m}]$.

Soient $x$ et $y$ dans une même classe modulo $P$. Il existe $z \in M$ tel que $x, y \in z+P$ (II, $\S 2 c$, définition IV de la congruence). On a : $x \not z, x-z \in P, y \not z, y-z \in P$. On en déduit $u(x)-u(z)=u(x-z) \in u(P)=(0)$, donc $u(x)=u(z)$; et on a de même $u(y)=u(z)$. Donc $u$ est constant sur toute classe modulo P. D'autre part, supposons que $u(L) \cap \mathcal{O}_{1} \subset u(L \cap \mathcal{L})$. De $L^{\prime}=\varphi(L)$ (qui est sous-monel, voir remarque 4 et théorème 1) on déduit :

$\bar{u}\left(L^{\prime}\right) \cap G_{1}=u(L) \cap G_{1} \subset u\left(L \cap O^{\prime} \subset u(L) \cap u\left(G_{)} \subset u(L) \cap G_{1}=\bar{u}\left(L^{\prime}\right) \cap O_{1}\right.\right.$. Donc $\bar{u}$ vérifie $\left[\mathrm{m}, \mathrm{L}^{\prime}\right]$. Le reste de la démonstration est trivial.

Théorème 3 : Soit $u: M \rightarrow M$, un homomorphisme de A-monels, vérifiant $[\mathrm{m}, M]$. $u(M)$ et $M / K$ er $u$ sont des $A$-monels isomorphes.

Avec les notations du théorème 2, posons $P=$ Ker $u$. On sait que $\bar{u}$ est un homomorphisme vérifiant $[\mathrm{m}, M /$ Ker $u$ ]. Son image $\operatorname{Im} \bar{u}=u(M)$ est un sous-monel de $M_{1}$ (théorème $1,3^{\circ}$ ). Enfin le noyau de $\bar{u}$ est nul par construction, et (lemme 4) $\bar{u}$ est injectif. Donc $\bar{u}$ se restreint (définition 2) en un isomorphisme de $M / \operatorname{Ker} u$ sur $u(M)$.

Corollaire : Soit $u: M \rightarrow M$, un homomorphisme surjectif de A-monels, tel que $u(\mathcal{O}) \supset \bigcup_{1}$. Alors $M_{1}$ et $M /$ Ker $u$ sont isomorphes.

Theorème 4 : Soient $M$ un A-monel, $K$ un sous-monel et la surjection canonique $\varphi: M \rightarrow M / K$. Soit $\mathscr{L}$ l'ensemble des sous-monels $L$ de $M$, contenant $K$, et $\mathscr{L}^{\prime}$ celui des sous-monels $L^{\prime}$ de $M / K^{\circ}$ (1) L'extension de $\varphi$ aux ensembles de parties induit une bijection de $\mathscr{L}^{\prime}$ sur $\mathscr{L}^{\prime}$. (2) Les A-monels $M / L$ et $(\mathrm{M} / \mathrm{K}) /(\mathrm{L} / \mathrm{K})$ sont isomorphes.

Pour tout sous-monel $L$ considéré, $\varphi$ vérifie $[\mathrm{m}, \mathrm{L}]$ (remarque 4), d'ou la bijection grace au théorème 1. D'autre part, posons $M^{\prime}=M / K^{\prime}, L^{\prime}=L / K$ et $v=\varphi^{\prime} \circ \varphi, \varphi^{\prime}$ étant la surjection canonique $M^{\prime} \rightarrow M^{\prime} / L^{\prime} \cdot \varphi$ et $\varphi^{\prime}$ étant 
des quasi-morphismes surjectifs, $v$ aussi. Par construction des quotients, $\boldsymbol{G}^{\prime}=\varphi(\boldsymbol{G})$ est le milieu de $M^{\prime}$, et $\mathcal{O}^{\prime \prime}=\varphi^{\prime}\left(\boldsymbol{G}^{\prime}\right)=\varphi^{\prime} \circ \varphi(\boldsymbol{G})$ est celui de $M^{\prime} / L^{\prime} \cdot$ En particulier, si $v(x)=\varphi^{\prime} \circ \varphi(x)$ (avec $x \in M$ ) n'est pas dans $G^{\prime \prime}$, il en résulte $\varphi(x) \notin \mathcal{O}^{\prime}$. Donc, les conditions $v(x) \notin G^{\prime \prime}, v(y) \notin \mathcal{O}^{\prime \prime}, v(x) \not v^{\prime}(y)$ entrainent $\varphi(x) \notin \mathcal{O}^{\prime}, \varphi(y) \notin \mathcal{G}^{\prime}, \varphi(x) \not \# \varphi(y)$, d'ou $x \not \# y$. Donc, $v$ est un homomorphisme surjectif vérifiant $[m, M]$. Son noyau est $\left(\varphi^{\prime} \circ \varphi\right)^{-1}(0)=\varphi^{-1}\left(L^{\prime}\right)=L$, ce qui montre l'isomorphisme annoncé.

Corollaire : Soit $u: M \rightarrow M$ un homomorphisme de A-monels, surjectif et tel que $u(\mathcal{O}) \supset G_{1}$. Soient $\mathcal{L}$ l'ensermble des sous-monels $L$ de $M$, contenant $K=$ Ker $u$, et $\mathcal{L}_{1}$ l'ensemble des sous-monels $L_{1}$ de $M_{1}$.

(1) L'extension de $u$ aux ensembles de parties induit une bijection de $\mathscr{L}_{0}$ $\operatorname{sur} \mathscr{L}_{1}$. (2) si $u(L)=L_{1}$ (donc $L=u^{-1}\left(L_{1}\right)$ ), $L_{1}$ et $L / x$ sont $i$ somorphes, et $M / L$ et $M_{1 / L}$ sont isomorphes.

En effet, par le théorème $3, M_{1}$ et $M / K$ sont isomorphes.

Théorème 5 : Soient $K$ et $L$ deux sous-monels d'un A-monel $M$, de milieu $O$. Si K (resp. L) est comparable a $O$, les A-monels $K+L / K$ et $\mathrm{L} / \mathrm{X} \cap \mathrm{L}$ sont isomorphes.

On peut se borner au cas ou $M=K+L$ : si $K$ (resp. $L$ ) est comparable a $O$, il l'est aussi à $O \cap(K+L)$. Considérons les applications canoniques $\mathrm{i}: \mathrm{L} \rightarrow \mathrm{K}+\mathrm{L}$ et $\varphi: \mathrm{K}+\mathrm{L} \rightarrow \mathrm{K}+\mathrm{L} / \mathrm{K}$, et posons $u=\varphi \circ i$. $\varphi$ étant un homomorphisme, $u$ aussi, car il est obtenu par restriction de $\varphi$ a $L$. Puisque $K$ (ou J) est comparable à $G$, tout $x \in X+I$ s'écrit (II, 2 b, démonstration du th. 1) $x=k+1$ (avec $k \in K, I \in L, k \# I$ ), et 1 'on a $\varphi(x)=\varphi(k+1)=\varphi(1)=u(1)$; donc $u$ est surjectif. Notons $\Omega=G \cap L$ et $U^{\prime}$ les milieux respectifs de $L$ et $K+L / K$. Considérons les divers cas.

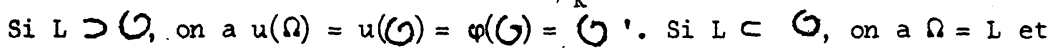
$K+L / K=u(L)=\varphi(L) \subset \varphi(\mathcal{O})=\mathcal{O}^{\prime}$; donc $u(\Omega)=\mathcal{O}^{\prime}$. si $\mathrm{K} \supset \mathcal{O}$, on $a \mathcal{O}^{\prime}=(0)$, donc $u(\Omega) \supset \mathcal{O}^{\prime}$. Si $k \subset \mathcal{O}$, tout $z \in \mathcal{U}$ s'écrit $z=k+1$, avec $k \in K$ et

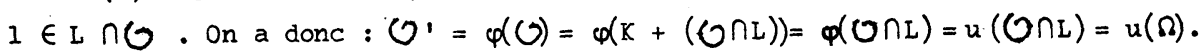
Donc $u$ vérifie les hypothèses du corollaire du théorème 3 et $K+L / K$ est isomorphe à $L /$ Ker $u$. Le noyau de $u$ est : Ker $u=\{I \in L ; \varphi(I)=0\}=\{I \in L ; I \in K\}=X \cap L$.

Exemple contraire 2 : Dans l'exemple contraire 1 ci-dessus, avec les notations du théorème 5 , $u$ est surjectif. Par contre, $(0)=u(\Omega) \neq \mathcal{U}^{\prime}=K+L / K$; enfin $L / L \cap K$ qui est isomorphe a $L, n^{\prime}$ 'est pas i somorphe, a $\mathrm{K}+\mathrm{L} / \mathrm{K}$.

Exemple contraire 3 : Dans l'exemple contraire au théorème 1, chap. II, $\S 2$ b, I'homomorphisme composé $u: F \rightarrow F+G \rightarrow F+G / G$ n'est pas 
surjectif. En effet, la classe modulo $G$ de $(a+b, a+b) \in \mathcal{O}(F+G)$ diffère de toute image $u(x), x \in F$.

Définition 3 : Soient $\mathrm{A}$ un anel, $\mathrm{M}$ un $\mathrm{A}$-monel, et $\mathrm{P}$ une partie non vide de $M$. On appelle annulateur de $P$ l'ensemble Ann $(P)=(0): P=\{a \in A ; \forall x \in P, a x=0\}$.

Il est clair que cet annulateur est un idéal.

Lemme 5 : Soient $M$ un A-monel, et $\Im$ un idéal de A. Pour que $M$ soit un A/ monel, il faut et il suffit que I'annulateur de $M$ contienne $\Im$. Si Ann $(M) \supset \Im$, pour tout $\alpha \in A / \Im$ et tout $x \in M$, prenons a $\in \alpha$ et posons $\alpha \mathrm{x}=\mathrm{a} \mathrm{x}$. Cette définition est indépendante du choix de a dans $\alpha$ : Si $a^{\prime} \in \alpha$, il existe $b \in \alpha$ tel que $a, a^{\prime} \in b+\Im_{0}$ De $a \not b$ et $a-b \in \Im \subset \operatorname{Ann}(M)$, on déduit $a x-b x=(a-b) x=0$; on a de même $a^{\prime} x=b x$, d'où $a x=a^{\prime} x$. Les autres vérifications sont immédiates.

Dans les résultats suivarts concernant les anels, les vérifications sont identiques à celles des théorèmes homologues sur les monels, sauf pour les propriétés liées à la multiplication. Mais la démonstration de ces dernières est immédiate. On notera $A_{\text {et }} A_{1}$ deux anels de milieux respectifs $\Omega$ et $\Omega_{1}$, dans ce qui suit, sauf à la remarque 5 bis.

Théorème 1 bis : Soit $u: A \rightarrow A$, un quasi-morphisme (d'anels). 1 - Pour tout idéal (resp. sous-anel) $\Im_{1}$ de $A_{1}, \bar{u}^{-1}\left(\Im_{1}\right)$ est un idéal (resp. sous-anel) de A. En particulier, le noyau Ker $u=\bar{u}^{-1}(0)$ de $u$ est un idéal. 2 - Si $B$ et X sont deux parties de $A$, on a $u(B X) \subset u(B) u(X)$ 。 on a l'égalité si $u$ est un homomorphisme vérifiant $\vec{u}^{-1}\left(\Omega_{1}\right) \subset \Omega_{0}$ 3 - Soit $B$ un sous-anel de $A$. Si $u$ est un homomorphisme vérifiant [m,B], $u(B)$ est un sous-anel de $A_{1} \cdot$ si, de plus, $u$ est surjectif, et si $B$ est un idéal de $A, u(B)$ est un idéal de $A_{1}$.

4 - Si u est un homomorphisme surjectif vérifiant [m,A], pour tout idéal $\mathfrak{\Im}$ contenant Ker $u, u(\Im)$ est un idéal.

Remarque 5 bis: L'image d'un annérde par un homomorphisme d'annéldes est un annérde. L'image d'un idéal par un homomorphisme surjectif d'annéides est un idéal.

Lemme 2 bis : Soit $u: A \rightarrow A$, un quasi-morphisme.

(1) Si $u$ est injectif, on a Ker $u=(0)$. (2) Si $u$ est un homomorphisme vérifiant $[m, A]$, et si Ker $u=(0)$, $u$ est injectif.

Lemme 3 bis : Soit $u: A \rightarrow A$, un homomorphisme bijectif. Les conditions suivantes sont équivalentes :

(1) $u$ est un isomorphisme ( soit $\Omega_{1} \subset u(\Omega)$ ).

(2) $\bar{u}^{-1}$ est un homomorphisme. 
(3) u vérifie $[m, A]$.

(4) $\bar{u}^{-1}\left(\Omega_{1}\right) \subset \Omega$.

Définition 2 bis : Un homomorphisme injectif $u: A \rightarrow A_{1}$ est dit un monomorphisme, s'il vérifie les conditions équivalentes suivantes :

(1) La restriction $v: A \rightarrow u(A)$ de $u$ est un isomorphisme.

(2) $u(A) \cap \Omega_{1} \subset u(\Omega)$.

(3) $u$ vérifie $[m, A]$.

(4) $\bar{u}^{-1}\left(\Omega_{1}\right) \subset \Omega_{0}$

Lemme 4 bis : Un homomorphisme de noyau nul et vérifiant [m,A]

est un monomorphisme.

Theorème 2 bis : Soient $\Im$ un idéal de A, et $\varphi$ la surjection $A \rightarrow A / g$. Si $u: A \rightarrow A_{1}$ est un quasi-morphisme (resp. homomorphisme) tel que $\mathfrak{g} \subset \mathrm{Ker} u$, il existe un quasi-morphisme (resp. homomorphisme) $\bar{u}: A_{\mathfrak{S}} \rightarrow \mathrm{A}_{1}$ tel que $u=\bar{u} \circ \varphi$.

2 - Si de plus u vérifie $[m, a]$ pour un idéal a contenant $\Im$, alors $\bar{u}$ vérifie $[m, a]$ ou $a$ est l'idéal $\varphi(a)$. Si de plus $u$ vérifie $[m, a]$ pour tout idéal $a$ contenant $\Im, \bar{u}$ vérifie $[\mathrm{m}]$.

Théorème 3 bis : Soit $u: A \rightarrow A_{1}$ un homomorphisme vérifiant [m,A]. Alors, $u(A)$ et $A / \operatorname{Ker} u$ sont des anels isomorphes.

Corollaire : Soit $u: A \rightarrow A_{1}$ un homomorphisme surjectif tel que $\Omega_{1} \subset u(\Omega)$. Alors, $A_{1}$ et $A / K e r ~ u$ sont isomorphes.

Theorème 4 bis : Soient $\Im$ un idéal de $A$, et $\varphi$ la surjection $A \rightarrow A / \mathfrak{\Im}$. Soient $\mathscr{L} I$ 'ensemble des idéaux a de $A$, contenant $\mathfrak{Y}$, et $\mathscr{L}$ ' celui des idéaux de $A / \Re^{\circ}$ (1) L'extension de $\varphi$ aux ensembles de parties induit une bijection de $\mathscr{L}$ sur $\mathscr{L}$. (2) Les anels A/a et $(\mathrm{A} / \mathrm{S} / \mathrm{k} / \mathrm{\jmath})$ sont isomorphes.

Corollaire : Soit $u: A \rightarrow A_{1}$ un homomorphisme surjectif tel que $\Omega_{1} \subset u(\Omega)$. Soient $\mathscr{L}_{l}$ 'ensemble des idéaux a de A, contenant $K=$ Ker $u$, et $\mathscr{L}_{1} I^{\prime}$ 'ensemble des idéaux $a_{1}$ de $A_{1} \cdot$ (1) L'extension de $u$ aux ensembles de parties induit une bijection de $\mathscr{L}$ sur $\mathscr{L}_{1}$. (2) si $u(a)=a_{1}\left(\right.$ donc $\left.\bar{u}^{-1}\left(a_{1}\right)=a\right)$, $a_{1}$ et $a / K$ sont isomorphes, et $A / a$ et $A_{1 / a_{1}}$ sont isomorphes.

Théorème 5 bis : Soient $\boldsymbol{a}$ et $\boldsymbol{b}$ deux idéaux de A. Si a (resp. b) est comparable à $\Omega$, les anels $a+b / a$ et $b / a \cap b$ sont isomorphes.

Application : Soient $A$ un anel de milieu $\Omega, \Im$ un idéal de $A$, et $B$ le plus petit anel agglutiné, intermédiaire entre $A$ et $\bar{A}$, et dont le milieu $O$ contienne $\Im_{0}$ Il est clair que B est obtenu à partir de A par l'agrandissement à $\mathcal{O}=\Omega+\bar{\Im}$ du milieu $\Omega$ de A (et bien sar l'agrandissement corrélatif des 
groupes d'addibilité). Alors, les anels $\mathrm{A} / \mathfrak{\Im}$ et $\mathrm{B} / \sqrt{\mathfrak{I}}$ sont isomorphes, ce qui équivaut à dire que les anneaux $\overline{(\mathrm{A} / \mathfrak{I})}$ et $\overline{\mathrm{A}} / \mathrm{I}$ sont isomorphes comme anneaux quasi-gradués. En effet, soit $u: A \rightarrow B /{ }_{\mathscr{I}}$ la restriction à la partie $A$ de $B$ de la surjection $\varphi: B \rightarrow B / \sqrt{\Im} ; u$ est donc un homomorphisme d'anels. Tout $\beta \in B / \sqrt{\mathfrak{S}} s^{\prime}$ écrit $\beta=b+\overline{\mathfrak{J}}$, où $b \in B$, soit $b=a+\eta$ (avec $a \in A$, $\eta \in \overline{\mathfrak{J}}$, d'après la définition de $B)$; on a donc $\beta=a+\overline{\mathfrak{S}}$ et $u$ est surjectif. Le noyau de $u$ est $\operatorname{ker} u=\{a \in A ; a \in \bar{\Im}\}=\Im_{\text {. }}$ Enfin, tout élément du milieu de $B / \sqrt{\mathfrak{J}}$ est de la forme $\omega+\overline{\mathfrak{T}}$ avec $\omega \in \Omega$, donc est élément de $u(\Omega)$. Le corollaire du théorème 3 bis montre que $A / \Im$ et $B / \sqrt{\Im}$ sont isomorphes.

De même, soit $\bar{M}$ un module quasi-gradué sur un anneau quasi-gradué $\bar{A}$, et soient $M$ et $A$ les monel et anel associés. Soit $\bar{L}$ un sous-module homogène de $\vec{M}$, et $\mathrm{L}$ le sous-monel des éléments homogènes de $\bar{L}$. Alors, les $\bar{A}$-modules quasigradués généralisés $\overline{(M / L)}$ et $\bar{M} / \bar{L}$ sont isomorphes.

$\$ 2$ - OPERATIONS SUR LES IDEAUX.

Les démonstrations de $a, b, c$, sont voisines de celles de [6] pages $147,177,219$ respectivement.

a-Formules :-

Soient $\mathrm{R}$ un anel et $a$ et $b$ deux idéaux de $R$. Le produit $a b$ de ces idéaux est 1 'idéal engendré par 1 'ensemble des produits ab (avec $a \in a, b \in b$ ), et leur somme $a+b$ est l'idéal engendré par $a \cup b(I I, \S 2$ b). On appelle radical de a et l'on note $\sqrt{a}$, l'ensemble des $x \in R$ tels qu'une puissance de $x$ soit dans $R$. Le radical de a est un idéal. On a les résultats suivants, où $a, b$, b' sont des idéaux de $R$, et où $\left\{a_{i}\right\}_{i \in I}$ désigne une famille

quelconque d'idéaux de $\mathrm{R}$ :

(2) $\quad a \cap b \supset a b$

(3) $\lambda(a b) \supset(\lambda a) b \quad$ (avec $\lambda \in \bar{R}, \lambda a$ désignant l'idéal engendré par

l'ensemble des produits $\lambda a$, où a $\in a$ agglutine $\lambda$ )

$$
\begin{aligned}
\sqrt{\sqrt{a}} & =\sqrt{a} \\
\sqrt{a+b} & =\sqrt{\sqrt{a}+\sqrt{b}}
\end{aligned}
$$

$$
\sqrt{\bigcap_{i \in I} a_{i}} \subset \bigcap_{i \in I} \sqrt{a_{i}} \text {. Il y a égalité lorsque I est fini. }
$$

(8) Lorsque $b \subset a$, on $a: \sqrt{a / b}=\sqrt{a / b}$

(9) Lorsque I est fini, on $a:\left(\bigcap_{i \in I} a_{i}\right): b=\bigcap_{i \in I}\left(a_{i}: b\right)$ 
(10) $(a: b): b^{\prime}=a: b b^{\prime}$

(11) Pour $n$ entier $\geqslant 1$, on $a:((a+b) / a)^{n}=\left(a+b^{n}\right) / a$

(12) Rappelons la loi modulaire (Voir II, $\S 2$ b)

Montrons par exemple la relation (1). L'idéal $a\left(b+b^{\prime}\right)$ est

engendré par I'ensemble des termes :

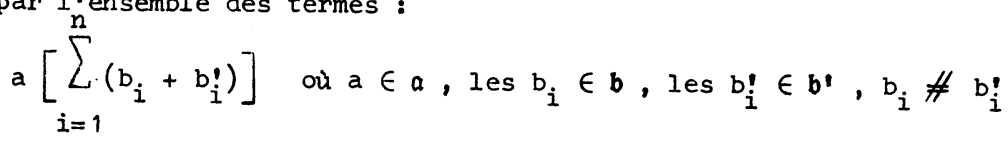

pour tout $i$, les parenthèses ont un total dans le milieu $\Omega$ de $R$, sauf une au plus

(II, $\S 2$ b). Les axiomes de distributivité montrent que le produit par a

est $\sum_{i=1}^{n}\left(a b_{i}+a b_{i}^{\prime}\right)$ avec les conditions analogues d'addibilité. Ceci

montre $a\left(b+b^{\prime}\right) \subset a b+a b^{\prime}$. Inversement, $a b+a b^{\prime}$ est engendré par

$a b \cup a b^{\prime}$, qui est contenu dans le premier membre.

Soient $R$ et $S$ deux anels de milieux respectifs $\Omega$ et $\Omega \mathbf{a}$, a et $\mathbf{b}$

deux idéaux de $R$, et $f: R \rightarrow S$ un quasi-morphisme de noyau $K$. (On sait que $f(a)$

$n^{\prime}$ 'est pas forcément un idéal). Outre le théorème 1 bis (III, $\S 1$ ), on a les

résultats suivants :

(13) $\quad f(a+b) \subset f(a)+f(b)$

(14) $\quad f(a \cap b) \subset f(a) \cap f(b)$.

Il y a égalité dans cette dernière formule si $\mathrm{K} \subset a$, et si $f$ est

un homomorphisme vérifiant $f(a \cap b) \supset \Omega^{\prime} \cap f(a) \cap f(b)$.

Les formules précédentes sont vraies dans le cas où $f$ est un quasi-morphisme ou homomorphisme de A-monels, $R$ et $S$ désignant alors des $A$-monels, et $a$ et $\mathfrak{b}$ des sous-monels de $R$.

$$
f(a: b) \subset f(a): f(b)=\{X \in S ; \forall Y \in f(b), X Y \in f(a)\}
$$

(16) $\quad f(\sqrt{a}) \subset \sqrt{f(a)}$.

Il y a égalité dans ces deux dernières formules si $f$ est un

homomorphisme surjectif qui vérifie $\bar{f}^{-1}\left(\Omega^{\prime}\right) \subset a \cdot\left(\right.$ resp. $\left.f(\Omega)=\Omega^{\prime}\right)$

Montrons, par exemple, ce demier résultat concemant (15).

Si $x \in f(a): f(b)$, par surjectivité de $f, X$ s'écrit $f(x)$ avec $x \in R$; et pour tout $b \in b$, il existe $a \in a$ tel que $X f(b)=f(x b)=f(a)$. Ou bien $f(x b) \in \Omega^{\prime}$ et la condition $\bar{f}^{-1}\left(\Omega^{\prime}\right) \subset a$ implique $x b \in a$. Ou bien $f(x b) \in \Omega$, et $x b$ et a sont addibles car $f$ est un homomorphisme. Il en résulte $0=f(x b)-f(a)=f(x b-a), d^{\prime}$ où $x b-a \in \bar{f}^{-1}\left(\Omega^{\prime}\right) \subset a$, et enfin $x b \in a$. On a donc $X=f(x) \in f(a: b)$. 


\section{b-Idéaux comaximaux :}

Des idéaux $a_{1}, \ldots . \ldots, a_{n}$ d'un anel unitaire $R$ seront dits deux à deux comaximaux $s^{\prime} i l s$ sont distincts de $R$ et si $i \neq j$ implique $a_{i}+a_{j}=R$. Lorsque $n=2$, on dit simplement idéaux comaximaux. On déduit des formules (a) :

Lenme : Soient $b, a_{1}, \ldots, a_{n}$ des idéaux d'un anel unitaire $R$. $\left.1^{\circ}\right)$ Les $a_{i}$ sont deux à deux comaximaux si et seulement si leurs radicaux le sont.

$\left.2^{\circ}\right)$ Si $b$ est comaximal avec chaque $a_{i}$, il l'est avec leur intersection et avec leur produit.

$3^{\circ}$ ) Si les $a_{i}$ sont deux à deux comaximaux, on a :

$$
\bigcap_{i=1}^{n} a_{i}=\prod_{i=1}^{n} a_{i}
$$

\section{c - Idéaux étendus et contractés :}

Soient $R$ et $S$ deux anels unitaires, et $f: R \rightarrow S$ un quasi-morphisme d'anels unitaires. On notera $a, b$ des idéaux de $R$, et $\mathscr{U}, \boldsymbol{B}$ des idéaux de $S$. L'idéal $\mathscr{U}^{\mathcal{E}}=\bar{f}^{1}(\boldsymbol{U})$ est dit contraction de $\boldsymbol{U}$. L'idéal $a^{e}=S f(\mathfrak{a})$ engendré par $f(a)$ dans $S$ est dit extension de $a$.

$$
\text { Il est clair que } R^{e}=S, S^{C}=R,(0)^{e}=(0) \text { et que }(0)^{c} \text { est le }
$$

noyau de $f$. On montre facilement les formules suivantes :

(1) $\quad \boldsymbol{U} \subset \boldsymbol{D}$ implique $\boldsymbol{\vartheta}^{\mathrm{C}} \subset \boldsymbol{\otimes}^{\mathrm{C}}$

$$
a \subset b \text { implique } a^{e} \subset b^{e}
$$

(2) $\boldsymbol{e}^{\text {ce }} \subset \boldsymbol{u}$ et $a^{\text {ec }} \supset a$

En particulier, lorsque $f$ est $I$ 'injection canonique $R \rightarrow \bar{R}$, on a $a^{\text {ec }}=a$, car $a^{e}=\bar{a}$ et $\bar{a}$ et $\bar{a} \cap R=a ; d$ autre part, la relation $\boldsymbol{\ell}^{\mathrm{Ce}}=\boldsymbol{\ell}$ signifie que l'idéal $\ell$ de $\bar{R}$ est homogène.

(3) $\boldsymbol{y}^{\mathrm{Fec}}=\boldsymbol{U}^{f}$ et $a^{\mathrm{ece}}=a^{\mathrm{e}}$

(4) $\quad(\boldsymbol{u}+\mathfrak{x})^{\mathrm{C}} \supset \boldsymbol{x}^{\mathrm{C}}+\mathfrak{x}^{\mathrm{C}}$

$(a+b)^{e}=a^{e}+b^{e}$

(5) $\quad(2 \cap y)^{c}=e^{c} \cap \mathfrak{B}^{c}$

$(a \cap b)^{e} \subset a^{e} \cap b^{e}$

(5) $\quad(\mathscr{U}:)^{c}>\mathscr{U}^{c} B^{c}$

$(a b)^{e}=a^{e} b^{e}$

(7) $\quad(\boldsymbol{2}: \text { b })^{c} \subset \mathscr{2}^{c}: \mathfrak{b}^{\mathrm{C}}$

$(a: b)^{\mathrm{e}} \subset a^{\mathrm{e}}: b^{\mathrm{e}}$

(8) $\quad(\sqrt{\mathfrak{r}})^{c}=\sqrt{\mathscr{\ell}^{\mathrm{e}}}$ et $(\sqrt{a})^{e} \subset \sqrt{a^{e}}$ 
Par exemple, vérifions pour (6) l'inclusion $a^{e} b^{e} \subset(a b)^{e}$. L'idéal $\mathfrak{a}^{\mathrm{e}} \boldsymbol{b}^{\mathrm{e}}$ est par définition engendré par les produits $Y Z$ où $Y \in a^{e}=S f(a)$ et $Y=b^{e}=S f(b)$. Donc $Y$ et $Z$ s'écrivent $Y=\sum_{i}\left[\sum_{j} s_{i j} f\left(a_{i j}\right)\right]$ et $z=\sum_{k}\left[\sum_{l} t_{k I} f\left(b_{k I}\right)\right]$ avec les $s_{i j}$ et $t_{k I} \in s$, les $a_{i j} \in a$, les $b_{k l} \in b$, et avec les conditions habituelles (II, $\S 2 b$ ): les termes intérieurs à un crochet sont mutuellement addibles ; pour Y (et de même pour $\mathrm{Z}$ ), les crochets sauf un au plus ont un total dans le milieu. Dans le produit :

$$
\begin{aligned}
Y Z & =\sum_{i, k}\left[\sum_{j, I} s_{i j} t_{k l} f\left(a_{i j}\right) f\left(b_{k l}\right)\right] \\
& =\sum_{i, k}\left[\sum_{j, 1} s_{i j} t_{k l} f\left(a_{i j} b_{k l}\right)\right]
\end{aligned}
$$

les axiomes de distributivité des anels conservent ces conditions. Il en résulte $Y Z \in(a b)^{e}$, d'où le résultat annoncé.

Soit (E) (resp. (C)) l'ensemble des idéaux étendus (resp. contractés). $a \rightarrow a^{e}$ et $\boldsymbol{U} \rightarrow \mathscr{U}^{\mathrm{C}}$ sont des bijections réciproques entre (E) et (C). (E) est fermé pour l'addition et la multiplication des idéaux. (C) est fermé pour l'intersection, la division et le passage aux radicaux. Ces dernières propriétés résultent immédiatement des formules précédentes, sauf la fermeture de (C) pour la division, dont la démonstration est identique à celle, connue, pour les anneaux : plus précisément, si $a, b \in(c)$, on montre que $a: b=\left(a^{e}: b^{e}\right)^{c}$.

Soient trois anels unitaires $R, S, T$, et deux quasi-morphismes unitaires $f: R \rightarrow S$ et $g: S \rightarrow T$. Il y a associativité des extensions et des contractions : I'extension par g de l'extension par $f$ est l'extension par $g \circ f ;$ on a le résultat analogue pour les contractions. Le résul tat est évident pour les contractions, et se montre sans difficulté pour les extensions. Par suite, si un diagramme de quasi-morphismes unitaires est commutatif, le diagramme associé des extensions (resp. des contractions) est commutatif.

\section{$\S 3$ - IDEAUX PREMIERS, PRIMAIRES, MAXIMAUX.}

Définition 1. Un idéal $p$ d'un anel A sera dit :

(1) premier si et seulement si pour tous $a, b \in A$, les relations $a b \in p$, $a \notin p$ impliquent $b \in p$. 
(2) strictement premier si et seulement si pour tout $b \in A$ et pour tout $\alpha=\sum_{i} a_{i} \in \bar{A}^{b}$, les relations $\alpha b \in p$ et $a_{i} \notin p$ pour tout $i$, impliquent $b \in p$.

(3) largement premier si et seulement si pour tout $b \in A$ et pour tout $\alpha=\sum_{i} a_{i} \in \Lambda^{b}$, les relations $\alpha b \in p$ et $a_{i} \notin p$ pour tout $i$, impliquent $b \in p_{0}$ (3) implique (2), qui implique (1). (Les conditions (2) et (3) se confondent pour un annélde). Noter que ces conditions signifient respectivement : (1) que $I^{\prime}$ anel $A / p$ est sans diviseur de zéro; (2) que de plus, A/p est sans pseudo-diviseur de zéro; (3) que de plus, A/p est sans pseudo-diviseur large de zéro. En particulier, lorsque A est unitaire, (1) signifie que A/p est un quasi-domaine, et (3) qu'il est un domaine.

Noter que si un idéal homogène $\bar{p}$ de $\bar{A}$ est premier dans $\bar{A}, p$ est premier dans $\mathbf{A}$ des trois manières. Inversement, $p$ peut être premier dans $A$, sans que $\bar{p}$ le soit dans $\bar{A}$.

Theorème 1. Soit A un anel.

(1) Un idéal $p$ est premier si et seulement si, quels que soient les idéaux $a$ et $b$ tels que $a b \subset p, p$ contient $a$ ou $b$.

(2) En particulier, si $p$ premier contient $a^{n}$ (avec $n \geq 1$ ), il contient $a_{0}$ (3) Si l'idéal $\Im \neq A$ n'est pas premier, il existe deux idéaux $a$ et $b$ contenant strictement $\mathfrak{Y}$, et tels que $a b \subset \mathfrak{O}$

La démonstration résulte immédiatement des définitions (voir [6], p. 150).

Definition 2. Un idéal $q$ d'un anel A sera dit :

(1) primaire si et seulement si pour tous $a, b \in A$, les relations $a b \in q$, a $\notin q$ impliquent $I^{\prime}$ existence de $n \geq 1$ tel que $b^{n} \in q_{0}$

(2) strictement primaire si et seulement si pour tout $b \in A$ et pour tout $\alpha=\sum_{i} a_{i} \in \bar{A}^{b}$, les relations $\alpha b \in q$ et $a_{i} \notin q$ pour tout $i$, impliquent 2'existence de $n \geq 1$ tel que $b^{n} \in q$.

(3) largement primaire si et seulement si pour tout $b \in A$ et pour tout $\alpha=\sum_{i} a_{i} \in \Lambda^{b}$, les relations $\alpha b \in q$ et $a_{i} \notin q$ pour tout $i$, impliquent I'existence de $n \geq 1$ tel que $b^{n} \in q$.

Noter que (1) signifie que, dans $A / q$, tout diviseur de 0 est nilpotent, (2) que de plus tout pseudo-diviseur de 0 est nilpotent, etc... Theorème 2. Soit A un anel.

10 Si q est un idéal primaire (resp. strictement primaire, resp. largement primaire), son radical $p=\sqrt{q}$ est premier (resp. strictement premier, resp. largement premier). On dit alors que $q$ est primaire pour $p$, ou est p-primaire. 
(2० Soit $q$ un idéal p-primaire. Les relations $a b \in q$ et a $\notin q$ impliquent $b \in p_{0}$ 3. Soient $q$ un idéal p-primaire, et deux idéaux a et b. Les relations $a b \subset q$ et $a \varnothing q$ impliquent $b \subset p$.

Supposons $q$ primaire, $a b \in \sqrt{q}=p$ et $a \notin p$. On a donc, pour un entier $n \geqslant 1, a^{n} b^{n} \in q$ et $a^{n} \notin q_{0}$. Alors il existe $m \geqslant 1$ tel que $\left(b^{n}\right)^{m} \in q_{\text {, soit }} b \in p_{0}$

Lorsque $q$ est strictement primaire, $p=\sqrt{q}$ est premier. Pour tout $b \in A$, et tout $\alpha=\sum_{i} a_{i} \in \bar{A}^{b}$ tels que les $a_{i}$ soient hors de $p$ et deux à deux non addibles, et que $\alpha$ b soit dans $p_{1}$ il existe $n \geqslant 1$ pour lequel $(\alpha b)^{n} \in q_{0}$ La propriété fondamentale des anels montre que $b^{n}$ agglutine (simplement) la somme formelle $\alpha^{n}$. Les termes de cette somme formelle sont hors de $p$, puisque $p$ est premier, car ce sont des produits des éléments $a_{i} \notin$ p. Alors, $q$ étant strictement primaire, il existe $m \geqslant 1$ tel que $\left(b^{n}\right)^{m} \in q$, soit $b \in p_{0}$ Le cas largement primaire se traite de même, grâce aux axiomes $D$ des anels. I'assertion (2) est triviale, et implique (3) en l'appliquant à un élément a de $a_{\bullet} \cdot q_{0}$

Définition 3. Un idéal m d'un anel A sera dit maximai si et seulement s'il est maximal dans l'ensemble des idéaux distincts de A.

Cela signifie que $A / m$ possède seulement deux idéaux distincts (0) et $\mathrm{A} / \mathrm{m} \cdot$ (III, $\S 1$, théorème 4 bis)

Théorème 3. Soit $\mathrm{R}$ un anel, de graduation $\Delta$ associative, dont les seuls idéaux sont (0) et $R$, et tel que $R^{2} \neq(0)$. Alors, $R$ ou est un corpolde, ou est égal à son milieu, lequel a une structure sous-jacente de corps.

Lemme 1. Dans un anel $R$ dont les seuls idéaux sont (0) et $R$, et tel que $R^{2} \neq(0)$, pour tout $x \in R, x \neq 0$, on a $R x=R$.

On rappelle que $R x$ est l'idéal $\left\{\gamma x ; \gamma \in \Lambda^{x}\right\}$ des multiples de $x$. Cet idéal est ici (0) ou $R$. L'ensemble $x=\{x \in R ; R x=(0)\}=(0)$ : $R$ est un idéal. L'hypothèse $R^{2} \neq(0)$ montre que $X$ est différent de $R$, et comme $(0)$ et $R$ sont les seuls idéaux, on a $x=(0)$. Donc, pour tout $x \in R, x \neq 0$, $l$ 'idéal $\mathrm{Rx}$ est différent de $(0)$, donc égal à $\mathrm{R}$.

Lemme 2. Soit $D$ un monorde commutatif (loi notée multiplicativement)

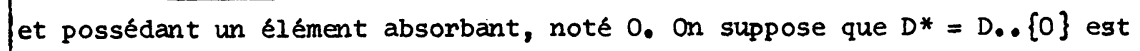
non vide. $S^{\prime} i l$ existe un système $S$ de générateurs de $D$ tel que $S S$ contienne $S$ pour tout $s \in S, s \neq 0$, alors $D^{*}$ est un groupe.

Prenons $a \in S, a \neq 0 ;$ puisque $s$ a $S$, il existe $e \in S$ tel que e $a=a_{\text {. Tout }} d \in D^{*}$ est multiple d'un élément non nul $s$ de $s$, soit $d=d^{\prime} s$ (avec $d^{\prime} \in D$ ). Puisque $S a \supset s$, il existe $t \in S$ tel que $t a=s$, donc que $d^{\prime} t a=d^{\prime} s=d_{0}$ On en déduit de $=d^{\prime} t a e=d^{\prime} t a=d_{0}$. Donc e est unité dans $D$. Pour tout s non nul dans $S, I$ 'hypothèse $S$ s $\supset$ montre qu'il existe 
$s^{\prime} \in s$ tel que $s^{\prime} s=e$, doric que s est inversible dans $s$. Alors, tout élément d de $D^{*}$, étant un produit d'éléments non nuls de $S$, est lui-même inversible.

Lemme 3. Un anel $R$ (commutatif), tel que $R^{*}=R_{\bullet}\left\{_{0}\right\}$ soit un groupe multiplicatif, ou est un corpolde, ou est égal à son milieu, lequel a une structure sous-jacente de corps.

Si le milieu $\Omega$ de $R$ est différent de (0), soit $x$ non nul dans $\Omega$; il est inversible et on a $1=x x^{\prime}$, avec $x^{\prime} \in R_{0}$. Grace à l'axiome $D_{5}$ des anels, ces conditions impliquent $1 \in \Omega$, donc aussi $R=\Omega$, et par suite $R$ est un corps.

Montrons le théorème. Notons $D$ le sous-monolde de $\Delta$ engendré par I'ensemble $\omega(R)=\omega\left(R_{\bullet} . \Omega\right) \cup\{0\}$ des grades des éléments de $R_{\bullet}$ Le lemme 1 montre que $R x=R$ pour tout $x \in R^{*}=R_{\bullet} \cdot\{0\}$. Cette condition montre que, pour tout $\alpha$ non nul de $\omega(R)$, on a : $\omega(R) \alpha \supset \omega(R)$. Le lemme 2 montre alors que $D^{*}$ (D est une graduation associative de $R$ ) est un groupe. $L^{\prime}$ anel $R$ est donc régulier. Il en résulte que la condition (relative à $I^{\prime}$ anel $R$ ) $R x=R$ pour tout $x \in R^{*}$, implique, dans le monolde $R, R x=R$ pour tout $x \in R^{*}$. (dans le monotde, $R x$ signifie $\{r \times ; r \in R\})$ Le lemme 2 montre que $R^{*}$ est un groupe, et le lemme 3 achève la démonstration.

Théorème 4. Soient $A$ un anel de graduation associative $\Delta$, et $m$ un idéal tel que $A^{2} \phi$. Pour que $m$ soit maximal, il faut et il suffit que $A / m$ ou soit un corpolde, ou soit égal à son milieu et aie une structure sous-jacente de corps.

$\mathrm{Si} \mathrm{A} / \mathrm{m}$ est un corpolde, il n'est pas de carré nul, et le théorème 4 bis (III, $\$ 1$ ) montre que $m$ est idéal maximal de $A$.

Inversement, les hypothèses montrent que $I^{\prime}$ anel $R=A / m$ est tel que $R^{2} \neq(0)$, a pour seuls idéaux (0) et $R$ (III, $\S 1$, théorème 4 bis) et admet la graduation associative $\Delta$ (voir II, $\S 2$ d). L'anel $R$ vérifie donc les conditions du théorème 3 , et est un corpolde.

Au lieu de $\Delta$ associative, il suffit de supposer que $A / m$ admette une graduation associative. Donc en particulier, il suffit que la partie stable $\Delta^{\prime}$ de $\Delta$ engendrée par $S=\{0\} \cup \omega\left(A_{\circ} \cdot(\Omega+m)\right)$ soit un monoide.

La dernière démonstration peut se faire directement : $m$ étant maximal, $\boldsymbol{m}$ contient l'idéal $A x+m=\Lambda^{x} x+m$ si et seulement si $A x \subset m$; on a dans le cas contraire $A x+m=A$. L'idéal $m: A=\{x \in A ; A x \subset m\}$ contient $m$ et en est différent, sinon on aurait $A^{2} \subset m_{0}$ Donc on $a m: A=A$, et $A x+m=A$ pour tout $x \in A_{0} \cdot m_{0}$ De ceci, et de $A^{2} f m$, on déduit $A=A^{2}$ et, pour tout $x \in A_{0} \ldots m_{\text {, on déduit } A}=(A x+m)^{2} \subset A x^{2}+m_{1}$ soit $A=A x^{2}+m_{0}$ Prenons a $\in A_{0} \cdot m ;$ puisque $A=A a^{2}+m$, il existe $\beta \in \Lambda^{a^{2}}$ tel que $a \equiv a^{2} \beta(m)$, donc aussi $a \equiv\left[a\left(a^{2} \beta\right)\right] \beta \quad(m)$. Comme $\Delta$ est associative, $a^{2}$ agglutine $\beta^{2}$ (II, $\S 3$, conséquence du corollaire 1) et on obtient $a \equiv$ a e (m) en posant $e=a^{2} \beta^{2} \in A$. 
Ensuite, de $A a+m=A$, pour tout $y$ de $A$ on déduit $I^{\prime}$ existence de $\gamma \in \Lambda^{a}$ tel que $y \equiv a \gamma(m)$, donc que e $y \equiv$ e a $\gamma=a \gamma=y$, et e est unité modulo mo Enfin, pour tout $x \notin m_{2} A=A x+m$ montre $I^{\prime}$ existence de $\delta \in \Lambda^{x}$ tel que $\mathrm{e} \equiv \mathrm{x} \delta(\mathrm{m})$, donc tel que $\mathrm{e}=\mathrm{e}^{2} \equiv(\mathrm{x} \delta)^{2}$. Puisque $\Delta$ est associative, $\mathrm{x}$ agglutine $\delta^{2}$. En posant $x^{\prime}=x \delta^{2} \in A$, on obtient $e \equiv x x^{\prime}(m)$, et $x$ est inversible modulo m. La démonstration s'achève par celle du lemme 3 . Corollaire : Soient $\mathrm{A}$ un anel de graduation associative $\Delta$, et $\mathbf{m}$
un idéal maximal tel que $\mathrm{A}^{2} \Rightarrow \mathrm{m}$. Alors, $\mathrm{m}$ est largement premier.

Remarque 1. Dans un anel unitaire, tout idéal propre a est contenu dans un idéal maximal. En effet, l'ensemble des idéaux propres contenant $a$, ordonné par inclusion, est inductif et contient un élément maximal (lemme de Zom).

Remarque 2. Dans un anel, I'intersection de tous les idéaux premiers est l'ensemble $\sqrt{(0)}$ des éléments nilpotents. En effet, tout idéal premier contient $\sqrt{(0)}$. Inversement, si u n'est pas nilpotent, I'ensemble $E$ des idéaux propres qui ne contiennent aucune puissance de $u$ est inductif et contient (0). Soit $p$ un élément maximal de $E$ (lemme de Zorn). Pour tous $x, y \notin p$, il existe $m, n \geq 1$ tels que $u^{m} \in p+(x)$ et $u^{n} \in p+(y)$, donc tels que $u^{m+n} \in p+(x y)$. Puisque $u^{m+n} \notin p$, on a $x y \notin p ; p$ est premier et ne contient pas $u$.

Théorème 5. Soient $p$ et $q$ deux idéaux d'un anel. A. $p$ est premier et $q$ est $p$-primaire si et seulement si :

(1) $q \subset p$

(2) pour tout $b \in p$, il existe $m \geqslant 1$ tel que $b^{m} \in q$

(3) pour tous $a, b \in A$, les relations $a b \in q$ et $a \notin q$ impliquent $b \in p$.

La démonstration est celle connue pour les anneaux (voir [6], p. 153). Noter que la relation (3) équivaut à ( $\left.3^{\prime}\right)$ : pour tous $a, b \in A$, les relations $a b \in q$ et $b \notin p$ impliquent $a \in q$.

Corollaire 1. Soient $p$ et $q$ deux idéaux d'un anel unitaire A. Supposons que :

(1) $q \subset p_{0}$

(2) pour tout $b \in p$, il existe $m \geqslant 1$ tel que $b^{\mathbf{n}} \in q_{0}$

(3) $p$ est maximal.

Alors, $p$ est premier et $q$ est $p$-primaire.

Si on $a$ ab $\in p$ et $b \notin p$, puisque $p$ est maximal, on en déduit $A=p+(b)$; donc on peut écrire $1=\sum_{i}\left(p_{i}+\gamma_{i} b\right)$ somme finie ou, pour tout $i$, $p_{i} \in p, \gamma_{i} \in \bar{A}^{b}$ (en fait, on devrait écrire $\gamma_{i} \in \Lambda^{b}$, mais on se ramène aisément au cas où les $\left.\gamma_{i} \in \overline{\mathrm{A}}^{\mathrm{b}}\right), p_{i} \# \gamma_{i} b$, et où les parenthèses ont un total dans le milieu $\Omega$, sauf une au plus. Pour tout $i$, soit $n_{i} \geq 1$ tel que 
$p_{i}^{n_{i}} \in q$, et soit $n=\sum_{i} n_{i}$. Alors $1=1^{n}=\left[\sum_{i}\left(p_{i}+\gamma_{i} b\right)\right]^{n}$. En développant, on obtient (axiomes $D$ et propriété fondamentale des anels) une somme de termes, addibles et tous situés dans $\Omega$ sauf un au plus, chacun de la forme $t_{j}=\Pi_{i}\left(p_{i}+\gamma_{i} b\right)^{m_{i}}$ où $\sum_{i} m_{i}=n$, et où $l^{\prime}$ un des exposants $m_{i_{0}} \geq n_{i_{0}}$. Un tel terme, développé à son tour, contiendra un multiple $q_{j} \in q$ de $p_{i_{0}}$, et un élément de l'idéal (b) (axiomes $D$ et propriété fondamentale). On a donc $1=\sum_{j} t_{j}=\sum_{j}\left(q_{j}+\delta_{j} b\right)$, avec $q_{j} \in q, \delta_{j} \in \Lambda^{b}$, et avec les conditions d'addibilité habituelles. On en déduit $a=\sum_{j}\left(a q_{j}+\delta_{j} a b\right)$, donc $a \in q$.

Corollaire 2. Dans un anel unitaire, tout idéal maximal $\mathfrak{m}$ est premier, et ses puissances sont m-primaires.

En effet, $q=m^{k}$ vérifie les conditions du corollaire 1 .

Théorème 6 . Soient $p$ et $q$ des idéaux d'un anel A. 10 Une intersection finie d'idéaux p-primaires (resp. strictement p-primaires) est p-primaire (resp. strictement p-primaire)

$2^{\circ}$ Si $p$ est maximal, une somme et un produit finis d'idéaux p-primaires sont p-primaires

$3^{\circ}$ Soit a un idéal non contenu dans q. Si $q$ est p-primaire, $q$ : a est p-primaire. Soient $q_{1}, \ldots, q_{n}$ des idéaux $p$-primaires d'un anel $A$ et $Q=\bigcap_{i} q_{i}$. On applique le théorème 5 . Si ab $\in Q$ et si $a \notin Q$, il existe $i_{0}$ tel que $a \in q_{i}$; alors $a b \in q_{i}$ entraine $b \in p$. Si, de plus, les $q_{i}$ sont strictement primaires, soient $b \in A$ et $\alpha=\sum_{j} a_{j} \in \bar{A}^{b}$ tels que $\alpha b \in Q$ et $a_{j} \notin Q$ pour tout $j$. Il existe donc $i_{1}$ tel que $a_{1} \notin^{\prime} q_{1}$. Posons $\alpha=\alpha^{\prime}+\alpha^{\prime \prime}$, ou la somme formelle $\alpha^{\prime}$ (resp. $\alpha^{\prime \prime}$ ) est formée des termes $a_{j} \notin q_{i}$ (resp. $\left.a_{j} \in q_{i}\right)$, et est agglutinée par b. Alors, on $a: \alpha^{\prime} b=\alpha b-\alpha^{\prime \prime} b \in q_{i_{1}}$; comme $\alpha^{\prime}$ contient au moins $a_{1}$, et comme $q_{i_{1}}$ est strictement p-primaire, $b$ est dans $p$.

(2) résulte du corollaire 1 du théorème 5. Pour (3), appliquons le théorème 5. On $a(q: a) a \subset q$ et $a \not q$, donc (théorème 2) on $a \quad q: a \subset p$. On sait que $q$ : a contient $q$. Puis, si $c b \in q$ : a et si $c \notin q: a$, il en résulte l'existence de $a \in a$ tel que ca $\notin q$; alors, cba $\in q$ montre que $b \in p$. Donc $q$ : a est $p$-primaire.

Théorème 7. Soient $R$ et $S$ deux anels de milieux respectifs $\Omega$ et $\Omega_{1}$, et $f: R \rightarrow S$ un homomorphisme surjectif tel que $f(\Omega)=\Omega_{1}$. Pour tout idéal a de $R$, contenant Ker $f$ (resp. pourtout idéal el de $S$ ), les idéaux a et $f(a)$ 
(resp. $\bar{f}^{1}(\mathfrak{U})$ et $\left.\mathscr{U}\right)$ sont ensemble maximaux, ou premiers, ou strictement
premiers, ou largement premiers, ou primaires, ou strictement primaires, ou
largement primaires. Si l'idéal $q$ contient Ker $f$ et est $p$-primaire, alors $f(q)$
est $f(p)$-primaire.

En effet, chacune des propriétés envisagées pour ces idéaux

a et $f(a)$. équivaut à une propriété des anels $\mathrm{R} / \boldsymbol{a}$ et $\mathrm{S} / \mathrm{f}(\boldsymbol{a})$. Or, ces anels sont isomorphes (III, $\S 1$, corollaire du théorème 4 bis). La dernière assertion résulte de (III, $\S 2$ a, formules (15)).

Théorème 8. Soient $R$ et $S$ deux anels, et $f: R \rightarrow S$ un quasi-morphisme. Si, dans $S, Q$ est un idéal $\beta$-primaire, alors $\beta^{C}$ est premier, et $Q^{C}$ est $P^{C}$-primaire.

La démonstration est triviale, mais elle ne s'étend pas au cas strictement primaire. Cependant, si $Q$ est idéal homogène de $\bar{R}$ (c'est-à-dire $Q=\overline{(Q \cap R)})$, lorsque $Q$ est strictement (resp. largement) primaire, il en est de même de $Q \cap R$.

Remarque 3. Si un idéal $\mathscr{U}$ de $S$ est intersection $\mathscr{U}=\bigcap_{i=1}^{n} Q_{i}$ d'idéaux primaires $Q_{i}$, il en est de même (III, $\S 2 c$, formule (8)) de son contracté : $\hat{U}^{C}=\bigcap_{i=1}^{n} Q_{i}^{C}$. Au contraire, l'extension ne conserve pas forcément premier et primaire.

\section{$\$ 4$ - ANELS DE FRACTIONS.} a-Définitions :

Monordes de fractions. Soit $R$ un monorde donné (noté multiplicativement)., commutatif, et pouvant éventuellement contenir un élément absorbant (ou annulateur), noté 0 , et un élément neutre, noté 1 s'il est distinct de 0 . Soit $M$ une partie multiplicative de M, c'est-à-dire non vide, multiplicativement stable, et ne contenant pas 0 . Dans le monoide produit $C=R \times M$ (multiplication par composantes), pour tous $(a, s)$ et $\left(a^{\prime}, s^{\prime}\right) \in C$, on pose $(a, s) H\left(a^{\prime}, s^{\prime}\right)$ si et seulement $s^{\prime} i l$ existe $t \in M$ tel que $t s^{\prime} a=t s^{\prime}$. Il est clair que $H$ est une relation d'équivalence sur $C$, et est compatible avec la multiplication. On notera $R_{M}=C / H$ le quotient, qui est donc un monorde commutatif, et on notera $\frac{a}{s}$ la classe d'un couple $(a, s)$. $R_{M}$ est appelé le monorde des fractions de $R$ relatif à $M$. Il possède 1 'élément unité $\frac{s}{s}$ (avec $s \in M)$. Pour tout $m \in M$, et pour tout $\frac{a}{s} \in R_{M}$, on $a: \frac{m a}{m s}=\frac{a}{s}$. Enfin, si $0 \in R, R_{M}$ admet $I^{\prime a n n u l a t e u r ~} 0=\frac{0}{t}$ (avec $t \in M$ ); $\frac{a}{s}=0$ signifie qu'il existe $m \in M$ tel que $m a=0$. (Dans le cas singulier où $0 \in M$, la construction est encore valable, mais conduit à un monorde réduit à un seul élément).

Si l'on ne suppose plus que la multiplication est associative dans $R$, 
mais seulement que les éléments de $\mathbb{M}$ associent dans $\mathrm{R}$ (voir II, $\S 3 \mathrm{~d}$, lemme 4), et si l'on conserve les autres hypothèses, cela suffit pour permettre la même construction avec les mêmes démonstrations. En effet, tout produit d'éléments de $R$ ou deux facteurs au plus ne sont pas dans $M$, vérifie les conditions d'associativité; et seuls de tels produits interviennent dans les démonstrations. On obtient alors un ensemble $R_{M}$ de fractions avec une multiplication commutative, pas forcément associative, possédant un annulateur et un élément neutre.

Anels de fractions. Soient $R$ un anel de milieu $\Omega$ et $M$ une partie multiplicative de $R$ (avec $0 \notin M$ ) . Organisons le monolde $R_{M}$ en un anel (les axiomes $A_{1234}$ des anels sont déjà vérifiés) de la manière suivante : $\frac{a}{s}$ et $\frac{b}{t}$ sont dits addibles dans $R_{M}$ si et seulement $s^{\prime} i l$ existe $m \in M$ tel que mta \# msb ; la somme est alors $\frac{a}{s}+\frac{b}{t}=\frac{m t a}{m t s}+\frac{m s b}{m s t}=\frac{m t a+m s b}{m s t}$ qui est bien (car mst $\in M$ ) dans $R_{M^{\circ}} L^{\prime}$ 'addibilité est indépendante des représentants choisis pour $\frac{a}{s}$ et $\frac{b}{t}$ ( $s^{\prime} i l$ en est ainsi, l'indépendance pour la somme est immédiate) : supposons $\frac{a}{s}=\frac{a^{\prime}}{s^{\prime}}$, soit us'a $=u s^{\prime}$ (avec $u \in M$ ). De mta \#msb on déduit mtusa' = mtus'a \# msus'b, puis mus.ta' \# mus.s'b; donc $\frac{a^{\prime}}{s^{\prime}}$ et $\frac{b}{t}$ sont addibles, car mus $\in M_{0}$. Les axiomes $B_{12}$ des anels sont vérifiés. Le milieu $O$ sera l'ensemble des éléments $\frac{a}{s} \in R_{M}$ tels qu'il existe $m \in M$ vérifiant ma $\in \Omega$. En effet, pour tout $\frac{b}{t} \in R_{M}$, mta $\in \Omega$ implique mta \# msb (Axiome $B_{3}$ ). si $\frac{a}{s} \# \frac{b}{t} \# \frac{c}{u}$ et si $\frac{b}{t} \notin \circlearrowleft$, c'est-à-dire $\mathrm{xb} \notin \Omega$ pour tout $\mathrm{x} \in \mathbb{M}$, les relations mta \#msb et nub \#ntc (avec $\mathrm{m}, \mathrm{n} \in \mathbb{M}$ ) impliquent mtnua \# (msnu)b \# msntc. Puisque msnub $\notin \Omega$, on a (mnt)ua \# (mnt)sc, donc $\frac{a}{s} \# \frac{c}{u}\left(\right.$ Axiome $\left.B_{4}\right)$. Donc $R_{M}$ est la réunion de domaines d'addibilité qui ont deux à deux $\mathcal{O}$ seulement en commun. On vérifie immédiatement que chaque domaine est un groupe additif abélien dont $0=\frac{0}{\mathrm{~s}}$ est le zéro commun. L'associativité résulte de celle de $\mathrm{R}$ grâce au :

Lemme 1 ( $\mathrm{d}^{2}$ addition) : Soient $\mathrm{n}$ fractions de $\mathrm{R}_{\mathrm{l}}$ deux à deux addibles. On peut les réduire au même dénominateur de manière que les nouveaux numérateurs soient addibles.

Pour $n=2$, c'est la définition. Soient $(n+1)$ fractions, soient $\frac{a_{1}}{m}, \ldots, \frac{a_{n}}{m}$ les $n$ premières supposées déjà réduites (avec les $a_{i} \in R$ deux à deux addibles), et soit $\frac{b}{s}$ la dernière. Pour tout $i, \frac{a_{i}}{m} \not \frac{b}{s}$ implique I'existence de $t_{i} \in M$ tel que $t_{i} s a_{i} \# t_{i} m$ b. Posons $t=t_{1} t_{2} \ldots . . t_{n}$. Pour tout $i$, on $a: t s a_{i} \# t m$ b. Alors $t$ n $s \in \mathbb{N}$ est le dénominateur commun cherché. 
Soient $\frac{a}{s}, \frac{b}{t}, \frac{c}{u}$ telles qu'il existe $m \in M$ vérifiant mta \#msb. on en déduit $m(u t) c a \# m(u s) c b$, donc $\frac{c a}{u s}$ et $\frac{c b}{u t}$ sont addibles (Axiome $D_{1}$ ). La distributivité du produit par rapport à la somme est alors aisée à vérifier. Enfin, si $\frac{b}{t} \in R_{M}$ et $\frac{a}{s} \in \mathcal{O}$, il existe $m \in M$ tel que $m a \in \Omega$; on en déduit $m(b a)=b(m a) \in \Omega$, donc $\frac{b a}{t s} \in \mathcal{O}$ (Axiome $\left.D_{5}\right) . R_{M i}$ est donc un anel commutatif et unitaire, appelé anel des fractions de $R$ relatif à la partie multiplicative $M$.

Remarque 1 : Si $R$ est un annérde, $R_{M}$ est un annérde. (C'est évident par définition du zéro et du milieu $\mathcal{O}$ de $\left.R_{\mathrm{M}}\right)$.

Fixons un $s \in M$ (par exemple $s=1$ si $1 \in M$ ) et considérons $I^{\prime}$ application $h: R \rightarrow R_{M}$ définie par $h(a)=\frac{s a}{s}$. Cette définition est indépendante du choix de s. On voit facilement que $h$ est un quasi-morphisme d'anels. En particulier, $a \in \Omega$ entraine sa $\in \Omega$, donc $h(a) \in O ; a \# b$ entraine $s^{2} a \# s^{2} b$, donc $h(a) \# h(b)$. Lorsque $R$ est unitaire, $h$ est un quasimorphisme d'anels unitaires. Lorsque $R n^{\prime}$ 'a pas d'élément unité, $R_{M}$ a encore un élément unité, mais $h n^{\prime}$ est pas unitaire. Pour tout $m \in M, h(m)=\frac{s m}{s}$ admet $\frac{s}{s m}$ pour inverse. Tout élément de $R_{M} s^{\prime e ́ c r i t ~}$

$\frac{a}{m}=\frac{s a}{s} \frac{s}{s m}=h(a) h(m)^{-1}=\frac{h(a)}{h(m)}$.

Lemme 2. Pour tous $a, b \in R$, la relation $h(a) \# h(b)$ équivaut à h'existence de $m \in \mathbb{M}$ tel que ma \#mb. En particulier, $h(a)=h(b)$ équivaut à p'existence de $m \in M$ tel que $h(m a-m b)=0$, donc de $t \in M$ tel que ta $=t b$.

Ce lemme est évident. Rassemblons ces divers résultats (I'unicité sera montrée plus loin):

Théorème 1. Soient $R$ un anel, et $M$ une partie multiplicative de $R$ (non vide et ne contenant pas 0 ). Il existe un anel unitaire $R_{M}$ et un quasimorphisme (unitaire si $\mathrm{R} l$ l'est) $h: \mathrm{R} \rightarrow \mathrm{R}_{\mathrm{M}}$ tel que :

(1) Ker $h=\{a \in R ; \exists m \in \mathbb{M}, \operatorname{ma}=0\}$

(2) Pour tout $m \in M, h(m)$ est inversible

(3) Tout élément de $R_{M i}$ est de la forme $\frac{h(a)}{h(m)}$, avec a $\in R, m \in M$

(4) $\overline{\mathrm{R}}^{\mathrm{h}}=\overline{\mathrm{R}}^{\mathrm{M}}$.

Il y a unicité de $R_{M}$ à un isomorphisme près.

Théorème 2. Soient $R$ un anel et $M$ une partie multiplicative de $R$ (non vide et ne contenant pas 0 ). Soient $R_{M} l$ 'anel et $h$ le quasi-morphisme du théorème 1. Quels que soient l'anel unitaire $S$ et le quasi-morphisme (unitaire si $R I^{\prime}$ 'est) $g: R \rightarrow S$ tels que les éléments de $g(M)$ soient inversibles, il existe un quasi-morphisme unitaire unique $u: R_{M} \rightarrow S$ tel que $g=u \circ h$.

Pour tout $a \in R$, on doit poser $u(h(a))=g(a)$. Pour tout $x=\frac{a}{s} \in R_{M}$, on a $h(a)=h(s) x$, donc on doit avoir $g(a)=u(h(s) x)=g(s) u(x)$, soit enfin 
$u(x)=\frac{g(a)}{g(s)}$. Si $R$ est unitaire, $g=u \circ h$ nécessite $g(1)=u \circ h(1)=u\left(\frac{s}{s}\right)=\frac{g(s)}{g(s)}=1$. Ceci montre 1 'existence et l'unicité de I'application u.

Si $\frac{a}{s} \# \frac{b}{t}$, c'est-à-dire s'il existe $m \in M$ tel que mta \#msb, on a $g(m t) g(a) \# g(m s) g(b)$. Puisque $g(m s t)$ est inversible, donc régulier, $u\left(\frac{a}{s}\right)=\frac{g(a)}{g(s)}$ et $u\left(\frac{b}{t}\right)=\frac{g(b)}{g(t)}$ sont addibles. La vérification des trois autres conditions pour que $u$ soit un quasi-morphisme unitaire est alors immédiate. Enfin, $g=u$ o $h$ montre que Ker $g$ contient Ker $h$ et que $\bar{R}^{g}$ est un agglutiné de $\overrightarrow{\mathrm{R}}^{\mathrm{M}}$.

Soient $S$ un anel de milieu $\mathcal{O}^{\prime}$ et $g: R \rightarrow S$ un quasi-morphisme vérifiant les mêmes conditions que $R_{M}$ et $h$ dans le théorème 1 . Soit $u$ le quasimorphisme associé à g par le théorème 2. Par la condition (4) (th. 1), deux éléments $a, b$ de $R$ sont addibles par $M$ si et seulement s'ils sont addibles par $h$ (resp. g) ; donc $h(a) \# h(b)$ équivaut à $g(a) \# g(b)$. Puisque les éléments inversibles d'un anel sont réguliers, $\frac{a}{s} \# \frac{b}{t}$ équivaut à $\frac{g(a)}{g(s)} \# \frac{g(b)}{g(t)}$. La même condition implique que $h(a) \in \mathcal{O}$ équivaut à $g(a) \in \mathcal{O}^{+}$, donc que $\frac{a}{\mathrm{~s}} \in \mathcal{O}$ équivaut a $\frac{g(a)}{g(s)} \in \mathcal{O}^{\prime}$. Donc u est un homomorphisme, vérifiant $u(\mathcal{O})=\mathcal{S}^{\prime} \mathcal{O}^{\prime}$. La condition (2) sur $s$ et $g$ montre que $u$ est surjectif. Soit $\frac{a}{s} \in R_{M}$ tel que $0=u\left(\frac{a}{s}\right)=\frac{g(a)}{g(s)}$; cela entraine $g(a)=0$; la condition (1) pour $g$ montre $l$ 'existence de $m \in M$ vérifiant $m a=0$, et on a $\frac{a}{s}=0$. Les anels unitaires $R_{M}$ et $S$ sont donc isomorphes (III, $\S 1$, lemme 3 bis), ce qui finit de démontrer le théorème 1.

Remarque 2. Dans les conditions du théorème 1, si. $M^{\prime}$ est une partie multiplicative contenant $M$, et si tout $m^{\prime} \in M^{\prime}$ est produit d'un élément de $M$ par un élément inversible de $R$, on a $R_{M}=R_{M}$. Le remplacement de $M$ par $M^{\prime}$ respecte en effet les quatre conditions.

Lemme 3. Si $R$ est un anel fort, $R_{M}$ est fort. Pour tout $x \in R$ tel que $\frac{x}{m} \notin \mathcal{O}$, soit $i=i(x)$ l'indice de quasirégularité de $x$. Supposons que, pour $n \geq i$ et pour $\frac{a}{s}$ et $\frac{b}{t} \in R_{M}, \frac{a}{s}\left(\frac{x}{m}\right)^{n}$ et $\frac{b}{t}\left(\frac{x}{m}\right)^{n}$ soient addibles et $n$ 'appartiennent pas a $O^{t}$. Il existe $u \in M$ tel que utm ${ }^{n} x^{n}$ et $u^{n}{ }^{n} b x^{n}$ sont addibles et $n$ 'appartiennent pas à $\Omega$. La quasirégularité de $x$ montre que $\left(\mathrm{m}^{n-i} u\right) \mathrm{tm}^{i} a x^{i} \not\left(\mathrm{m}^{\mathrm{n}-i} u\right) \mathrm{sm}^{i} b x^{i}$, donc que $\frac{x}{m}\left(\frac{x}{m}\right)^{i} \# \frac{b}{t}\left(\frac{x}{m}\right)^{i}$. Le lemme suivant est évident.

Lemme 4 . On a : $(\bar{R})_{M}=\overline{\left(R_{M}\right)}$.

Notons les quasi-morphismes $h: R \rightarrow R_{M}$ et $\bar{h}: \bar{R} \rightarrow(\bar{R})_{M}$. Une somme formelle $\alpha \in \bar{R} . . R$ est dans le noyau de $\bar{h}$ si et seulement si elle est annulée par au moins un élément $m \in M$, qui est un diviseur ou un pseudo-diviseur de zéro. Ainsi, la localisation détruira bien des sommes formelles. D'autre part, Ker $\bar{h}$ 
contient $\overline{\operatorname{Ker} h}$, et en est différente en général.

Une graduation de $R_{M}$ dans le cas associatif.

Lemme 5 . Soit un anel $R$ de graduation $\Delta$, et $M$ une partie multiplicative de $R$ (avec $0 \notin M)$. Si les éléments de $\omega(M)$ associent dans $\Delta, \Delta_{\omega(M)}$ est une graduation de $\mathrm{R}_{\mathrm{M}}$. Si $\Delta$ est associative, $\Delta_{\omega(\mathrm{M})}$ est une graduation associative de $\mathrm{R}_{\mathrm{M}}$.

Notons $A=R_{M}$ et $\Delta^{\prime}=\Delta_{\omega}(M)$. Soient $\Omega$ et $\mathcal{O}$ les milieux respectifs de $R$ et $A$. Ecartons le cas où $M$ rencontre $\Omega$, donc où $R_{M}$ est égal à son milieu, et est gradué trivialement par $\{0\}=\Delta^{\prime}$. Lorsque $M \cap \Omega$ est vide, on a $0 \notin \omega(M)$. Le grade de $\frac{a}{s} \in A$ sera par définition $\omega^{\prime}\left(\frac{a}{s}\right)=\frac{\omega(a)}{\omega(s)} \in \Delta^{\prime}$, qui est indépendant du représentant choisi : en effet, les relations ts'a = tsa' $\notin \Omega$, (avec $t \in M$ ) impliquent $\omega(t) \omega\left(s^{\prime}\right) \omega(a)=\omega\left(t s^{\prime} a\right)=\omega\left(t s a^{\prime}\right)=\omega(t) \omega(s) \omega\left(a^{\prime}\right)$. Alors, à tout $\frac{\alpha}{\sigma} \in \Delta^{\prime}$, on fait correspondre l'ensemble $\frac{A}{\sigma} \frac{\alpha}{\sigma}$ formé de $O$ et (s'il y a lieu) des fractions $\frac{a}{s} \notin \mathcal{O}$ de grade $\frac{\alpha}{\sigma}$. Quels que soient les grades $\frac{\alpha}{\sigma}, \frac{\beta}{\tau} \in \Delta^{\prime}$ tels que $\frac{A_{\alpha}}{\sigma} \frac{\beta}{\tau} \not \subset \mathcal{O}^{\prime}$, prenons deux fra cions $\frac{a}{s}, \frac{b}{t} \notin 0$, de grades respectifs $\frac{\alpha}{\sigma}$ et $\frac{\beta}{\tau}$, et vérifiint $\frac{a b}{s t} \notin 0$. De ab $\notin \mathcal{O}$ et st $\notin \mathcal{O}$, on déduit

$\omega^{\prime}\left(\frac{a b}{s t}\right)=\frac{\omega(a b)}{\omega(s t)}=\frac{\omega(a) \omega(b)}{\omega(s) \omega(t)}=\omega^{\prime}\left(\frac{a}{s}\right) \omega^{\prime}\left(\frac{b}{t}\right)$. Donc $\Delta^{\prime}$ est bien une graduation de A. (I, $\S 3$ et II, $\S 2$ a).

b - L'extension par $h: R \rightarrow R_{M}$

Lemme 6. Soient $R$ un anel de milieu $\Omega$, $M$ une partie multiplicative de $R$ (avec $0 \notin M$ ) et a un idéal de $R$.

(1) Si $a^{e}$ est l'extension de a à $R_{M}$, tout élément de $a^{e}$ peut s'écrire $\frac{a}{m}$, avec $a \in a$ et $m \in M$.

(2) $\frac{b}{t} \in a^{e}$ (avec $b \in R, t \in M$ ) équivaut à l'existence de $m \in M$ tel que $m b \in a$.

(3) En particulier, $\Omega^{e}$ est le milieu de $R_{M^{*}}$ Tout $x \in a^{e}=R_{M} f(a) s^{\prime}$ écrit (II, $\S 2$ b) :

$x=\sum_{j}\left[\sum_{i} \frac{b_{i}^{j}}{t_{i}^{j}} \cdot \frac{s a_{i}^{j}}{s}\right]$ où les $a_{i}^{j} \in a$, où le même $a_{i}^{j}$ peut intervenir plusieurs fois, où pour tout $j$ fixé, les fractions contenues dans le crochet d'indice $j$ sont addibles deux à deux, et où les crochets ont un total dans le milieu $\boldsymbol{O}$, sauf un au plus. Le lemme 1 permet de remplacer chaque crochet par une fraction $\frac{c_{j}}{u_{j}}$, oùchaque $c_{j}$ est dans $a$, comme combinaison linéaire simple des $a_{\dot{I}}^{j}$. Le lemme 1 permet de remplacer $\sum_{j} \frac{c_{j}}{u_{j}}$ par $x=\frac{a}{m}$, ou a est dans $a$, comme 
combinaison linéaire simple des $c_{j} \in a_{\text {. }}$

Si une fraction $\frac{b}{t}$ (avec $b \in R, t \in M$ ) est dans $a^{e}$, ce qui précède montre qu'elle est égale à une fraction $\frac{a}{s}$, avec a $\in$ a. Donc, il existe $u \in M$ tel que (us) b = uta $\in$ a.

Définition. Soient $\mathrm{R}$ un anel, $\mathrm{M}$ une partie multiplicative de $\mathrm{R}$ (avec $0 \notin \mathrm{M}$ ) et a un idéal de $\mathrm{R}$. On appelle fermeture divisible de a dans $\mathrm{R}$ relativement à $M, I$ 'ensemble $a_{d}=\{x \in R ; \exists m \in M$ tel que $m x \in a\}$. Lorsque $a_{\mathrm{d}}=\boldsymbol{a}$, on dit que $\mathrm{M}$ est premier à $\mathbf{a}$.

on voit d'abord que

$$
a_{d}=\bigcup_{m \in M}(a:\{m\})=\bigcup_{m \in M}(a:(m)) \text {. }
$$

Propriétés. (1) Pour que $a_{d}=R$, il faut et il suffit que a rencontre M. Soit $s \in M$; si $R=a_{d}$, il existe $m \in M$ tel que $m s \in a_{\text {. Inversement, }}$ $t \in M$ implique $a:(t)=R$.

(2) Si h est le quasi-morphisme $R \rightarrow R_{M}$, on a $a_{d}=a^{e C}$.

En. effet, $x \in a_{d}$ signifie qu'il existe $m \in M$ et $a \in a$ tels que $m x=a$; on a donc $h(x)=\frac{a}{m} \in a^{e}$. Inversement, si $h(x) \in a^{e}$, le lemme 6 entrafne l'existence de $a \in a$ et de $m \in M$ tels que $h(x)=\frac{a}{m}=\frac{h(a)}{h(m)}$, et (lemme 2) il existe $t \in M$ vérifiant $(\mathrm{tm}) \mathrm{x}=$ ta $\in a_{\text {. Dunc }} \mathrm{x}$ est dans $a_{\mathrm{d}}$.

(3) En particulier, $a_{d}$ est un idéal et contient Ker $h$.

(4) Pour que $M$ soit premier à $a$, il faut at il suffit que $a^{\text {eC }}=a$, ou que $a:(m)=a$ pour tout $m \in M$. Alors, le lemme $\sigma$ montre que $\frac{b}{t} \in a^{e}$ si et seulement si $b \in a$.

Théorème 3. Soient $\mathrm{R}$ un anel, $\mathrm{M}$ une partie multiplicative de $\mathrm{R}$ (avec $(0 \in M)$, a un idéal de $R$, et $h: R \rightarrow R_{M^{*}}$ (a) $a^{\text {ec }}=a_{d}$ est $l^{\prime}$ 'ensemble des $x \in R$ tels que $m x \in a$ pour un $m \in M$. $a^{e}$ est distinct de $R_{M}$ si et seulement si $M \cap a$ est vide.

(b) a est un idéal contracté et vérifie $a=a^{e c}$ si et seulement si $M$ est premier à a. Alors, $\frac{b}{t} \in a^{e}$ (avec $b \in R, t \in M$ ) équivaut à $b \in a$.

(c) Tout idéal $\Im$ de $R_{M}$ est étendu, et on a $\Im=\Im^{c e}$.

(d) L'application $a \mapsto a^{e}$ est une bijection de I'ensemble (c) des idéaux contractés sur I'ensemble des idéaux de $R_{M}$, et conserve I'intersection, Ia ãivision et la prise de radical des idéaux.

(a) et (b) étant connus (Propriétés 2, 1, 4), montrons (c). Pour tout $\frac{x}{m} \in \mathfrak{J}$, on a $\frac{m x}{m} \in \mathfrak{J}$, donc $x \in \mathcal{J}^{\mathrm{C}}$. Il en résulte $\mathfrak{J} \subset \mathfrak{J}^{\mathrm{ce}}$; I'inclusion inverse est connue (III, $\S 2 c$ ). Enfin, (d) résulte de (c) et des considérations de 'III, $\S 2 c)$.

Nous savons (III, $\S 3$, théurème 8 ) que la contraction d'un idéal P-primaire est $\boldsymbol{B}^{\mathrm{C}}$ - primaire. 
Théorème 4. Soient $\mathrm{R}$ un anel, $M$ une partie multiplicative, et $q$ un idéal p-primaire disjoint de $M$.

(a) $p$ est disjoint de $M, p$ et $q$ sont des idéaux contractés et contiennent le noyau de $h$.

(b) $q^{e}$ est primaire pour $p^{e}$.

Puisque $\mathbb{M}$ et $q$ sont disjoints, toute puissance d'un $x \in \mathbb{M}$ est dans $M$, et ne peut être dans $q$; cela entrâne $x \notin p$. Par suite, $M$ est premier à $p$, donc à $q$, et ces idéaux $p$ et $q$ sont contractés (th. $3, b$ ). La définition de p premier (resp. q-primaire) implique, grâce à la dernière assertion (b) du théorème 3 , que $p^{e}$ est premier (resp. $q^{e}$ est primaire). Par définition du radical, la même assertion montre que $\mathfrak{p}^{e}=\sqrt{q^{e}}$.

Corollaire 1. L'application $p \mapsto p^{e}$ est une bijection de l'ensemble des idéaux premiers contractés (c'est-à-dire disjoints de $M$ ) de $R$ sur 1 'ensemble des idéaux premiers de $R_{M}$.

Cela résulte des théorèmes 4 et 3 (c), de (III, $\S 2$ ) et de (III, $\S 3$, théorème 8).

Corollaire 2. Pour un idéal premier contracté $p$ donné, l'application $q \mapsto q^{e}$ est une bijection de $I^{\prime}$ ensemble des idéaux $p$-primaires de $R$ sur l'ensemble des idéaux $p^{e}$-primaires de $R_{M}$. Cette bijection conserve la division let l'intersection de deux idéaux.

La première assertioh résulte du théorème 4. La deuxième vient de ce que, si $q$ et $q^{\prime}$ sont $p$-primaires, leur intersection et leur quotient le sont aussi (sauf dans le cas trivial $q^{\prime} \subset q$, cas où les quotients sont $R$ et $R_{M}$ )

Remarque 3. (Transitivité) Soient $M$ et $M^{\prime}$ deux parties multiplicatives d'un anel $R$, telles que $M \subset M^{\prime}$ et $0 \notin M^{2}$, et $h$ (resp. $h^{\prime}$ ) le quasi-morphisme $R \rightarrow R_{M}$ (resp. $R \rightarrow R_{M^{\prime}}$ ). Alors les anels $\left(R_{M}\right)_{h}\left(M^{\prime}\right)$ et $R_{M}$, sont canoniquement isomorphes.

Il existe (th, 2) un quasi-morphisme unique $u: R_{M} \rightarrow R_{M}$, tel que $h^{\prime}=u \circ h$. Utilisons le théorème 1. On montre aisément (voir [6] p. 226) que $h\left(M^{\prime}\right)$ est une partie multiplicative de $R_{M}$, que le noyau de $u$ est l'ensemble des éléments de $R_{M}$ annulés par au moins un élément de $h\left(M^{\prime}\right)$, que les éléments de $u\left(R\left(M^{\prime}\right)\right)=h^{\prime}\left(M^{\prime}\right)$ sont inversibles dans $R_{M^{\prime}}$, et que tout élément de $R_{M^{\prime}}$

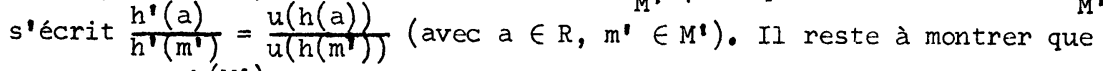

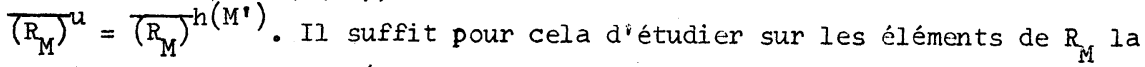
condition d'addibilité (resp. d'appartenance au milieu) dans ces deux agglutinés simples de $R_{M^{*}}$.

Soient $a_{1}$ et $b_{1}$ dans $R_{M}$, qui s'écrivent $a_{1}=\frac{h(a)}{h(s)}$ et $b_{1}=\frac{h(b)}{h(t)}$ 
(avec $a, b \in R$ et $s, t \in M)$. La condition $u\left(a_{1}\right) \# u\left(b_{1}\right)$ équivaut (car $h^{\prime}=u \circ h$ ) à $\frac{h^{\prime}(a)}{h^{\prime}(s)}=\frac{h^{\prime}(b)}{h^{\prime}(t)}$, donc à $l^{\prime}$ existence de $m^{\prime} \in \mathbb{M}^{\prime}$ tel que $m^{\prime}$ ta \#\# $m^{\prime} s b$. Cela implique $h\left(m^{0}\right) h(t) h(a) \not \# h\left(m^{1}\right) h(s) h(b)$, donc $h\left(m^{0}\right) a_{1} \not \# h\left(m^{0}\right) b_{1}$. Inversement, $h\left(m^{\prime}\right) a_{1} \# h^{\prime}\left(m^{\prime}\right) b_{1}$ implique $h\left(m^{\prime} t a\right) \# h\left(m^{t} s b\right)$, donc implique (lemme 2)

l'existence de $m \in M$ tel que $\left(\mathrm{mm}^{\prime}\right)$ ta $\#\left(\mathrm{~mm}^{2}\right) \mathrm{sb}$. Enfin, on applique $\mathrm{h}^{\prime}$ et on divise dans $R_{M}$, par l'inversible $h\left(m m^{\prime} t s\right)$ pour obtenir $\frac{h^{\prime}(a)}{h^{\prime}(s)}=\frac{h^{\prime}(b)}{h^{\prime}(t)}$.

Les conditions successives suivantes sont équivalentes : $a_{1}=\frac{h(a)}{h(s)}$ est dans le milieu de $\left(R_{M}\right)^{u} ; u\left(a_{1}\right)=\frac{h^{\prime}(a)}{h^{\prime}(s)}$ est dans le milieu de $R_{M^{\prime}}$; il existe $m^{\prime} \in M^{\prime}$ tel que $m^{\prime} x$ soit dans le milieu de $R$; il existe $n^{\prime} \in M^{\prime}$ tel que $h\left(n^{\prime} a\right)=h\left(n^{\prime}\right) h(a)$ soit dans le milieu de $R_{M} ; h(a)$ est dans le milieu

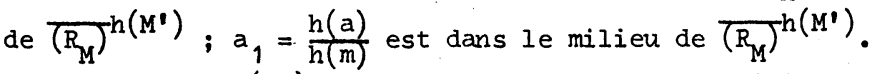

Donc $h\left(M^{\prime}\right) \subset R_{M}$ et $u$ vérifient les conditions du théorème 1 , et si on note $g$ le quasi-morphisme canonique $R_{M} \rightarrow\left(R_{M}\right)_{h}\left(M^{\prime}\right)$, il existe un isomorphisme

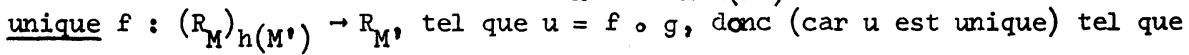
$h^{\prime}=f \circ g \circ h_{0}$

Alors, la transitivité des extensions (III, $\S 2 \mathrm{c}$ ) montre que $\rho$ induit, entre les ensembles d'idéaux de $\left(R_{M}\right)_{h}\left(M^{\prime}\right)$ et de $R_{M}$, une bijection telle que, pour tout idéal a de $R$, on ait $\quad f\left(\left(a^{e}\right)^{e}\right)=a^{e^{\prime}}$ (en notant e, e' et é les extensions respectives par $h, h^{\prime}$ et $\left.g\right)$.

Remarque 4. (Permutabilité des passages au quotient et à un anel de fractions) Soient $R$ un anel, $M$ une partie multiplicative de $R$ (avec $0 \notin M$ ), et $a$ un idéal disjoint de $M$. Soit $a^{e} l$ 'extension de a par $h: R \rightarrow R_{M}$. Alors, les anels $R_{M / a}$ e et $(R / a){ }_{(M+a) / a}$ sont canoniquement isomorphes.

La démonstration est construite comme pour la remarque 3 . Notons $f$ et $f^{\prime}$ respectivement les homomorphismes $R \rightarrow R / a$ et $R_{M} \rightarrow R_{M / a}$. On montre aisément

(voir [6] p. 226) qu'il existe un quasi-morphisme unique $u: R / a \rightarrow R_{M / a} e$ vérifiant $u \circ f=f^{\prime} \circ h$, que $f(M)=(M+a) / a$ est une partie multiplicative de $R / a$ ne contenant pas zéro, que le noyau de $u$ est l'ensemble des $a_{1}=f(a) \in R / a$ annulés par au moins un élément $m_{1}$ de $f(M)$, que tout élément de $u(f(M))$ est inversible dans $R_{M / a}{ }^{e}$, et que tout élément de $R_{M} / a$ e peut s'écrire $\frac{u\left(a_{1}\right)}{u\left(m_{1}\right)}$, avec $a_{1} \in R / a$ et $m_{1} \in f(M)$. Pour appliquer le théorème 1 à $f(M) \subset R / a$ et $u$, montrons aussi que $(\overline{R / a})^{u}=(\bar{R} / \mathfrak{a})^{f(M)}$.

Soient $\Omega, \mathcal{O}$ et $\mathcal{O}_{1}$ les milieux respectifs de $R, R_{M}$ et $R_{M} / a^{e}$. On a $\mathcal{O}_{1}=\left\{x+a^{e} ; x \in \mathcal{O}\right\}=\left(\mathcal{Q}+a^{e}\right) / a^{e}$. Mais le lemme 6 et la formule 4 de (III, § 2c) montrent $\mathcal{O}+a^{e}=\Omega^{e}+a^{e}=(\Omega+a)^{e}$, d'où $\mathcal{O}_{1}=(\Omega+a)^{e} / a^{e}$. Soit $a_{1} \in R / a$ tel que $u\left(a_{1}\right) \in \mathcal{O}_{1}$, et prenons un $a \in R$ tel que $a_{1}=f(a)$. 
La condition $u\left(a_{1}\right)=f^{\prime} \circ h(a) \in \mathcal{O}_{1}$ équivaut à $h(a) \in(\Omega+a)^{e}$, donc équivaut à l'existence de $m \in M$ tel que ma $\in \Omega+a$, donc enfin à l'existence de $f(m) \in f(M)$ tel que $f(m) f(a) \in(\Omega+a) / a^{*}$ Puisque $(\Omega+a) / a$ est le milieu de $R / a^{\prime}$ $a_{1}$ est dans le milieu de $\left.\overline{(R / a)}\right)^{f(M)}$.

Soient $a_{1}, b_{1} \in R / a$ et $a, b \in R$ tels que $a_{1}=f(a)$ et $b_{1}=f(b)$. Supposons $u\left(a_{1}\right)=f^{\prime} \circ h(a)$ et $u\left(b_{1}\right)=f^{\prime} \circ h(b)$ addibles et non situes dans $G_{1}$. Ceci équivaut ( $f^{\prime}$ est un homomorphisme) à $h(a)$ et $h(b)$ addibles et non situés dans $(\Omega+\mathfrak{a})^{e}$. Donc ceci signifie qu'il existe $m \in M$ tel que ma \#mb, et qu'on $a:$ sa, sb $\notin \Omega+a$ pour tout $s \in M_{0}$. f étant un homomorphisme, et $(\Omega+a) / a$ étant le milieu de $R / a$, ces conditions équivalent enfin aux suivantes : il existe $f(m) \in f(M)$ tel que $f(m) a_{1} \# f(m) b_{1}$ et, pour tout $f(s) \in f(M), f(s) a_{1}$ et $f(s) b_{1}$ ne sont pas dans le milieu de $R / a^{*}$

Donc $f(M) \subset R / a$ et $u$ vérifient les conditions du théorème 1 , et si $I^{\prime}$ on note $h^{\prime}$ le quasi-morphisme canonique $R / \mathfrak{a} \longrightarrow(R / \mathfrak{a}) f(M)$, il existe un isomorphisme unique $v:\left(R / a^{\prime} f(M) \longrightarrow R_{M} / \mathfrak{a}\right.$ e tel que $u=v \circ h^{\prime}$, donc (unicité de $u$ ) tel que $\rho^{\prime} \circ h=v \circ h^{\prime} \circ f$.

c-Exemples :

Donnons quelques exemples de parties multiplicatives dans un anel $R$ :

(1) $M$ est le complément dans $R$ d'un idéal premier $p_{0}$ Alors $R_{M}$ est noté $R_{p}$.

(2) $M$ est le complément dans $R$ de la réunion $\underset{i \in I}{U} p_{i}$ d'une famille quelconque d'idéaux premiers.

(3) $M$ est l'ensemble des puissances de $s$, où $s$ est un élément non nilpotent de $R$.

(4) $M$ est l'ensemble des éléments d'un sous-anel de $R$, non contenus dans un idéal premier de $R$.

(5) Etudions le cas où $M$ est le complément d'une réunion finie $\bigcup_{i=1}^{n} p_{i}$ d'idéaux premiers vérifiant les conditions : (a) pour tout $i=1, \ldots, n$ on a $p_{i} \not \underset{j \neq i}{U} p_{j}$;

(b) pour tout $i=1, \ldots, n$, si un idéal premier $\pi$ est tel que

$p_{i} \subset \pi \subset \bigcup_{j=1}^{n} p_{j}$, on a $p_{i}=\pi$.

Si la famille finie $\left\{p_{i}\right\}_{i=1, \ldots, n}$ donnée ne vérifie pas ces conditions,

on peut se ramener (avec le même $M$ ) à une famille qui les vérifie. En effet, considérons la famille $F$ des suites $S=\left\{\pi_{1}, \ldots, \ldots, \pi_{n}\right\}$ de $n$ idéaux premiers tels que pour tout $i=1, \ldots, n$, on ait $p_{i} \subset \pi_{i} \subset \bigcup_{j=1}^{n} p_{j}$. Ordonnons-la en posant $s^{\prime} \leqslant S^{\prime}=\left\{\pi_{1}^{\prime}, \ldots, \pi_{n}^{\prime}\right\}$ si et seulement si $\pi_{i} \leqslant \pi_{i}^{\prime}$ pour tout $i=1, \ldots, n$. Il est clair que l'idéal réunion d'une chatne croissante d'idéaux premiers contenant $p_{i}$ et contenus dans $\bigcup_{j=1}^{n} p_{j}$ est premier et vérifie les mêmes inclusions. Par quite, 
la famille $\mathrm{F}$ est inductive et (lemme de Zorm) possède un élément maximal $\left\{p_{1}^{\prime}, \ldots, p_{n}^{\prime}\right\}$. Les idéaux $p_{i}^{\prime}$ vérifient la condition (b). Enfin, pour avoir la condition (a), on supprime parmi eux les $p_{i}^{!}$superflus, et ceux qui restent vérifient encore la condition (b).

Lemme 7. Si $p_{1}, \ldots, p_{n}$ sont des idéaux premiers de $R$ vérifiant les conditions (a) et (b) ci-dessus, et si $M$ est le complément de $n$ $\bigcup_{i=1} p_{i}$, la famille des idéaux $p_{1}^{e}, \ldots, p_{n}^{e}$ est celle de tous les idéaux maximaux de $\mathrm{R}_{\mathrm{M}^{*}}$

Tout idéal $p_{i}^{e}$ est propre. Comme $R_{l i}$ est unitaire, $p_{i}^{e}$ est contenu dans un idéal maximal $\Im$ de $R_{M}$, et $\Im$ est premier (III, $\S 3$, remarque 1 et corollaire 2 du théorème 5). Donc, I'idéal $\Im^{\complement}$ est premier (III, $\S 3$, théorème 8 ) et vérifie $p_{i} \subset \mathcal{Y}^{c} \subset \bigcup_{j=1}^{n} p_{j}$. Par la condition (b), $p_{i}$ est l'idéal contracté $\mathcal{Y}^{\mathbb{S}}$ et on a enfin $p_{i}^{e}=\Im^{c e}=3$ Donc, chaque $p_{i}^{e}$ est maximal, et $p_{1}^{e}, \ldots, p_{n}^{e}$ vérifient les conditions (a) et (b) (III, $\S 4$, théorème 3 ). Soït un idéal maximal $\mathfrak{m}$ de $R_{M}$ (donc $\mathfrak{m} \subset \bigcup_{i=1}^{n} p_{i}^{e}$ ), mais distinct de tout $p_{i}^{e}$. Pour tout $i, \mathfrak{m}$ et $p_{i}^{e}$ étant maximaux et non comparables, sont comaximaux. Donc $\boldsymbol{m}$ et $\bigcap_{i=1}^{n} p_{i_{n}}^{e}$ sont comaximaux (III, $\S 2 b$ ). Alors, $R=\mathfrak{m}+\bigcap_{i=1}^{n} p_{i}^{e}$ serait contenu dans $\bigcup_{i=1} p_{i}^{e}$, ce qui est une contradiction.

Voici un exemple où la condition (b) n'est pas vérifiée. Soient I'anneau $\mathbb{Z}$ des entiers, et I'anneau $\bar{A}=\mathbb{Z}$ e $\oplus \mathbb{Z}$ a, où e est unité de $\bar{A}$, et où $a^{2}=$ a. Dans l'annérde $A=\mathbb{Z}$ e $U \mathbb{Z}_{\text {a }}$, les idéaux $p_{1}=\mathbb{Z}$ e et $p_{2}=2 \mathbb{Z}$ e U $2 \mathbb{Z}$ a sont premiers, non comparables, et leur réunion est un idéal premier.

\section{$\S 5$ - IDEAUX LARGES.}

a-Définitions.

Soient $\mathrm{A}$ un anel de milieu $\Omega$ et de graduation fixée $\Delta, \Im$ un idéal et $B$ un sous-anel de $A$, tous deux contenant $\Omega_{0}$

$\Im$ (resp. B) est dit large en grade $\alpha \in \Delta, s^{\prime}$ il contient tous les éléments de $A$ ayant ce grade, C'est-à-dire s'il contient $A_{\alpha}$, ou encore si $A_{\alpha}=\Im_{\alpha}$ (resp. $B_{\alpha}$ ) (la notation $\Im_{\alpha}$ signifie $\Im \cap A_{\alpha}$ ). Noter que $\Im$ est large sur tout grade nul ( $\alpha$ est dit nul lorsque $A_{\alpha}=\Omega$, et il est dit vide si de plus $\alpha \neq 0$; voir I, $\S 3$ ).

$\Im$ (resp. B) est dit faiblement large si, pour tout $\alpha \in \Delta, \Im_{\alpha}$ (resp. $\mathrm{B}_{\alpha}$ ) est égal à $\Omega$ ou à $\mathrm{A}_{\alpha}$, C'est-à-dire si $\Im$ (resp. B) est large en grade $\alpha$, pour tout $\alpha \in \omega(\Im)$ (resp. $\omega(B)$ ). 
Le sous-anel B est dit large, $S^{\text {il }}$ il est faiblement large et si, pour tous grades $\alpha$ et $\beta$ non vides, B large en grades $\alpha$ et $\beta$ implique B large en grade $\alpha \beta$.

L'idéal 3 est dit large, s'il est faiblement large et si, pour tout grade $\alpha$ non vide, $\Im$ large en grade $\alpha$ implique $\Im$ large en grade $\delta \alpha$, quel que soit $\delta \in \Delta$.

Lemme 1. (a) Soient $F$ et a deux idéaux de A. Si F' est faiblement large, on $\mathrm{a} F+a=\mathrm{F} \cup(a+\Omega)$.

(b) Si L et $\mathrm{L}^{\prime}$ sont des idéaux faiblement larges (resp. larges) de A, il en est de même de $L+L^{\prime}=L U L^{\prime}$.

(a) est trivial, car F contient $\Omega$, et (b) en résulte aussitôt. Remarque 1. La notion d'idéal large dépend de la graduation donnée $\Delta$,

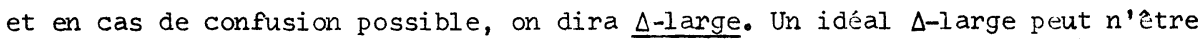
pas large pour une autre graduation $\Delta^{\prime}$. Cependant, si une graduation $\Delta^{\prime}$ prolonge (II, §4) une graduation $\Delta$, tout idéal $\Delta^{\prime}$-large est $\Delta$-large. Montrons-le: Pour un anel $A$, soit $\Delta_{0}$ sa graduation propre, soit $(\Delta, \theta)$ une graduation donnée, et soit $\left(\Delta^{\prime}, \theta^{\prime}=\varphi \circ \theta\right)$ une graduation prolongeant $\Delta$ (voir II, $\S 4$ ), donnée par $\varphi: \Delta^{\prime} \rightarrow \Delta$. Notons $B==^{-1}\left(\Delta_{0}^{*}\right)$ et $B^{\prime}=\varphi^{-1}(B)$. Les applications $\theta, \varphi$ et $\theta^{\prime}$ sont des bijections entre $\Delta_{0}^{*}$, B et $B^{\prime}$; pour tous $\alpha^{\prime}, \beta^{\prime} \in \Delta^{\prime}$, la relation $\varphi\left(\alpha^{\prime}\right) \varphi\left(\beta^{\prime}\right) \neq 0$ implique $\varphi\left(\alpha^{\prime}\right) \varphi\left(\beta^{\prime}\right)=\varphi\left(\alpha^{\prime} \beta^{\prime}\right)$ (voir II, $\S 4$ ). Soit L un idéal $\Delta^{\prime}$-large. Pour tout $\alpha \in \omega(L), \alpha \neq 0$ (donc $\alpha \in B$ ), et pour tout $\delta \in \Delta^{*}$, posons $\alpha^{\prime}=\bar{\varphi}^{-1}(\alpha)$ et $\delta^{\prime}=\bar{\varphi}^{-1}(\delta)$ (on sait que $\varphi$ est injective $\left.\operatorname{sur} \vec{\varphi}^{1}\left(\Delta^{*}\right)_{\bullet}\right)$; on a $A_{\alpha}=A_{\alpha^{*}} \subset L_{\text {. }}$ ou bien $\delta \alpha \notin B$, et $A_{\delta} \alpha=\Omega \subset I_{0}$ Ou bien $\delta \alpha \in B$, et $\delta \alpha \neq 0$ implique $\delta \alpha=\varphi\left(\delta^{\prime}\right) \varphi\left(\alpha^{\prime}\right)=\varphi\left(\delta^{\prime} \alpha^{\prime}\right)$. Donc on a $\mathrm{A}_{\delta \alpha}=\mathrm{A}_{\delta^{\prime} \alpha^{\prime}}$. Puisque $\mathrm{L}$ est $\Delta^{\prime}$-large et contient $\mathrm{A}_{\alpha^{\prime}}=\mathrm{A}_{\alpha}$, il contient $\mathrm{A}_{\delta} \alpha{ }$ Remarque 2. En particulier, tout idéal $\Delta$-large est aussi $\Delta_{0}$-large, si $\Delta_{0}$ est la graduation propre. On voit donc que les seuls idéaux d'un anel A qui pourront être larges sont ceux qui le sont déjà pour la graduation propre.

Remarque 3 . Lorsque la graduation $\Delta$ est associative, il existe une bijection évidente entre l'ensemble des idéaux $\Delta$-larges de A, et l'ensemble des $\Delta$-idéaux de $\Delta$. Dans le cas d'une graduation non-associative, la situation se complique un peu; nous nous bornerons, grâce à la remarque 2, à étudier le cas de la graduation propre:

Remarque 4. La graduation propre $\Delta_{0}$ possède une loi multiplicative partout définie, pas forcément associative. Une partie non vide $\Lambda$ de $\Delta_{0}$ est dite un idéal si et seulement si $\Delta_{0} \Lambda=\left\{\delta \alpha ; \delta \in \Delta_{0}, \alpha \in \Lambda\right\} \subset \Lambda$. Pour tout idéal $\Lambda$, il est donc clair que $\mathrm{A}_{\Lambda}=\bigcup_{\alpha \in \Lambda} \mathrm{A}_{\alpha}$ est un idéal $\Delta_{0}$-large de $\mathrm{A}$. Inversement, si $L$ est idéal $\Delta_{0}$-large de $A$, posons $\Lambda=\omega(L)=\{\omega(a) ; a \in L\}$. 
Pour tout $\alpha \in \Lambda, \alpha \neq 0$, et pour tout $\delta \in \Delta_{0}$ tels que $\delta \alpha \neq 0$, on a $A_{\delta \alpha} \neq \Omega$ puisque $\Delta_{0}$ est la graduation propre; mais L est large en grade $\delta \alpha$ car il est large en grade $\alpha \neq 0$. On a donc $\mathrm{L} \supset \mathrm{A}_{\delta \alpha} \neq \Omega$, d'où $\delta \alpha \in \Lambda$; et $\Lambda$ est un idéal de $\Delta_{0}$. Il existe donc une bijection évidente entre l'ensemble des idéaux de $\Delta_{0}$ et l'ensemble des idéaux larges de $A$.

Lemme 2. Soient 1 'anel $A$ de graduation $\Delta$, a et $L$ deux idéaux de $A$, et I'anel $\mathrm{A} / \mathrm{a}$ canoniquement gradué par $\Delta$ (II, $\S 2 \mathrm{~d}$ ). Si L est $\Delta-1$ arge (resp. faiblement large), alors $(L+a) / a$ est $\Delta$-large (resp. faiblement large) dans $A / a^{\circ}$ En particulier, si $\Omega$ est le milieu de $A$, pour qu'un idéal $L$ contenant $\Omega$ sait $\Delta$-large (resp. faiblement large) dans $A$, il faut et il suffit que $L / \Omega$ soit $\Delta-1$ arge (resp. faiblement large) dans $I^{\prime}$ annéide $A / \Omega$ •

Par contre, si l'idéal $\mathrm{H}$ contient $a$ et si $\mathrm{H} / \mathrm{a}$ est large dans $\mathrm{A} / \mathrm{a}$, $\mathrm{H} \mathrm{n}^{\prime}$ 'est pas forcément large dans A. (par exemple $a / a$ est large lorsque $a \supset \Omega$ ) Notons $A^{\prime} I^{\prime}$ 'anel $A / a, \Omega^{\prime}=(\Omega+a) / a$ son milieu, $L^{\prime}=(L+a) / a$ et $\omega^{\prime}(\mathrm{X})$ le grade $\mathrm{d}^{\prime}$ un élément $\mathrm{X}$ de $\mathrm{A} / \mathrm{a}^{*}$ Pour tout grade $\alpha \in \Delta$, on a $A_{\alpha}^{\prime}=\left(A_{\alpha}+a\right) / a$, et la condition $A_{\alpha}^{\prime} \neq \Omega^{\prime}$ équivaut à $A_{\alpha}+a \neq \Omega+a$, c'est-à-dire à $A_{\alpha} \cdot .(\Omega+a) \neq \phi$. Il est clair que si L est large en grade $\alpha$,

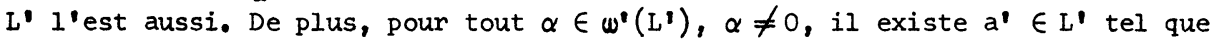
$\omega^{\prime}\left(a^{\prime}\right)=\alpha$; donc $a^{\prime}$ est la classe modulo a d'un élément a de $L, a \notin \Omega+a$, tel que $\omega^{\prime}\left(a^{\prime}\right)=\omega(a)=\alpha$, et L est donc large en grade $\alpha$. Par suite, pour tout $\delta \in \Delta, L$ est large en grade $\delta \alpha$, donc $L$ ' l'est aussi.

Pour toute partie $P$ de $A$, contenant $\Omega$, on appelle noyau large de $P$, et on note $P_{L}$, le plus grand idéal large de $A$, contenu dans $P$. Le lemme 1 montre l'existence du noyau large, comme réunion des idéaux larges contenus dans $P$. De même, on appelle noyau faiblement large de $P$, et on note $P_{F}$, le plus grand idéal faiblement large contenu dans $P$. On a $\Omega \subset P_{L} \subset P_{F} \subset P$.

Pour toute partie $P$ de $A$, on appelle enveloppe large (resp. faiblement large) de $P$, et on note $E I(P)$ (resp. FI(P)), le plus petit idéal large (resp. faiblement large) contenant $P$.

Soit $\Im$ un idéal de $A$, contenant $\Omega_{0}$ Si le noyau large (resp. faiblement large) de $\Im$ est $\Omega$, on dit que $\Im$ est un idéal étroit (resp. strictement étroit). $\Im$ est strictement étroit si et seulement si pour tout grade non vide $\alpha \in \Delta^{*}$, il existe $a \in A_{\alpha}$ tel que a $\not \Im$. L'idéal $\Im$ est étroit si et seulement si pour tout grade non vide $\alpha \in \Delta^{*}$, il existe un élément $\mathrm{b} \in \mathrm{A}$, b $K \mathcal{Y}$, dont le grade $\omega(\mathrm{b})=\beta$ est $\alpha$ ou un multiple de $\alpha$.

Lemme 3. Soient I'anel A de graduation $\Delta$ et de milieu $\Omega$, deux idéaux $a$ et $b$ de $A$ tels que $b \supset a \supset \Omega$, et $I$ 'annélde $A / a$ canoniquement gradué par $\Delta_{0}$ Si a est le noyau lange de $b$, alors $b / a$ est étroit. Inversement, si a est large et si $b / a$ est étroit, alors a est le noyau large de b. 
Prenons les notations du lemme 2 et désignons par $x^{\prime}, y^{\prime}, \ldots$. les classes modulo a de $x, y, \ldots$ Supposons que a soit le noyau large $b_{L}$ de $b$. Pour tout $\alpha \in \omega^{\prime}(b / a), \alpha \neq 0$, soit $x \in b / a ; x \neq 0 ;$ il existe $x \in b, x \notin a$, tel que $X=x^{\prime}$. Puisque $a=b_{L}$, il existe un élément $y \in A, y \notin b$, dont le grade est $\alpha$ ou un multiple de $\alpha$. Alors, $y^{\prime}$ est de même grade et $n^{\prime}$ appartient pas a $b / a$. Donc $b / a$ est étroit.

Inversement, remarquons d'abord que I'hypothèse a large n'est pas superflue, car $b / b$ est étroit quel que soit $b$. Supposons donc $a$ large et $b / a$ étroit. Pour tout $x \in b_{0} . a$, posons $\alpha=\omega(x)=\omega^{\prime}\left(x^{\prime}\right)$. Puisque $b / a$ est étroit, il existe un grade $\beta$, qui est $\alpha$ ou un multiple de $\alpha$, et il existe $Y \in A_{\beta}^{\prime}=\left(A / a_{\beta}\right.$ tels que $Y \notin b / a$. Soit alors $y \in A_{\beta}$ tel que $Y=y^{\prime} ; 1 a$ condition $y^{\prime} \in \mathrm{b} / \mathrm{a}$ implique $\mathrm{y} \notin \mathrm{b}$. Donc $a$ est le noyau large de $b$.

\section{b-Anels de fractions :}

Dans ce paragraphe $b$, on désigne par $R$ un anel de milieu $\Omega$ et de graduation $\Delta$, M une partie multiplicative telle que $0 \notin \mathbb{M}, \mathcal{O}^{\prime}$ le milieu de $R_{M}$, et on considere $l^{\prime}$ extension et la contraction par le quasi-morphisme $h: R \rightarrow R_{M^{*}}$ Dans la suite, I'hypothèse éventuelle que $\omega(M)$ associe dans $\Delta$ signifie que tout élément de $\omega(M)$ associe dans $\Delta$ (II, $\S 3 d_{2}$ lemme 4 ).

Lemme 4. Si $I^{\prime i d e ́ a l ~} \mathrm{~F}$ de $\mathrm{R}$ est faiblement large, alors $\mathrm{F}^{\mathrm{e}} \mathrm{l}^{\text {'est }}$ aussi. Si $\omega(M)$ associe dans $\Delta_{2}$ et si l'idéal $L$ de $R$ est $\Delta-l$ arge, alors $L^{e}$ est $\Delta_{\omega}(M)^{-l \text { arge. }}$

Pour la démonstration, on peut supposer que $F \cap M=\varnothing$, sinon $F^{e}=R_{M}$, et supposer que $R$ est un annérde, grace au lemme 2 .

Soit un élément non nul de $F^{e}$, qui peut s'écrire $\frac{a}{s}$ avec $a \in F$, $s \in M\left(I I I, \S 4 a\right.$, lemme 6). Soit $\frac{b}{t}$ une fraction non nulle de $R_{M}$, addible a $\frac{a}{s}$. Réduisons-les (III, $\S 4 a$, lemme 1) au mêne dénominateur $\frac{\mathrm{mta}}{\mathrm{mts}} \# \frac{\mathrm{msb}}{\mathrm{mst}}$ de manière que mta et msb soient addibles. Puisque a est dans $F$ et que $\frac{a}{s}$ est non nul, mta est élément non nul de $\mathrm{F}$; alors $\mathrm{F}$ faiblement large implique msb $\in F$, et enfin $\frac{b}{t}=\frac{m s b}{m s t} \in F^{e}$. Donc $F^{e}$ est faiblement large.

$D^{\prime}$ abord, $L^{e}$ est faiblement large. On notera $\omega^{\prime}(x)$ le grade d'un élément $x$ de $R_{M}$. Pour tout grade $\xi \in \omega^{\prime}\left(L^{e}\right), \xi \neq 0$, prenons un élément de $L^{e}$ ayant ce grade, soit $\frac{a}{s}$ vérifiant les conditions a $\in L$, a $\neq 0, s \in \dot{M}$ et $\xi=\frac{\alpha}{\sigma}$ (ou l'on pose $\alpha=\omega(a), \sigma=\omega(s)$ ). Pour tout grade $\frac{\alpha \beta}{\sigma \gamma}$ non nul (c'est-à-dire dont le groupe d'addibilité diffère de (0); I, $\S 3$ ) multiple de $\frac{\alpha}{\sigma}$, quel que soit l'élément (non nul) $\frac{c}{r}$ de $R_{M}$, de grade $\omega^{\prime}\left(\frac{c}{r}\right)=\frac{\gamma}{p}=\frac{\alpha \beta}{\sigma}$ (en posant $\omega(c)=\gamma$ et $\omega(r)=p)$, il existe $\mu \in \omega(M)$ tel que $\mu \sigma \gamma \gamma=\mu \rho \alpha \beta$. Noter que tout produit d'éléments de $\Delta$, où deux facteurs au plus ne sont pas dans $\omega(M)$, est associatif, puisque $\omega(M)$ associe dans $\Delta$. Prenons $m \in M$ de grade 
$\omega(m)=\mu$. Alors, $\frac{c}{r} \neq 0$ implique $m$ stc $\neq 0$, donc aussi

$\omega(m$ stc $)=\mu \sigma \tau \gamma=(\mu \rho \beta) \alpha$. Puisque $L$ large contient $a$, il est large en grade $\alpha=\omega(a)$, donc en grade $(\mu \rho \beta) \alpha$; donc on a m stc $\in L$, d'où $\frac{c}{r}=\frac{m \text { st } c}{m \text { st } r} \in L^{e}$. Ceci montre que $L^{e}$ est $\Delta_{\omega(M)}$-large.

Au contraire, la contraction d'un idéal faiblement large dans $R_{M}$. $n$ 'est pas forcément faiblement large dans $R$. On remarque d'abord que $\mathcal{O}=\Omega^{\mathrm{e}}$ et que $\mathcal{O}^{\mathrm{C}}=\Omega^{\mathrm{eC}}=\Omega_{\mathrm{d}}$ (III, $\S 4 \mathrm{~b}$, lemme 6 , propriété 2$)$. Ensuite, on a :

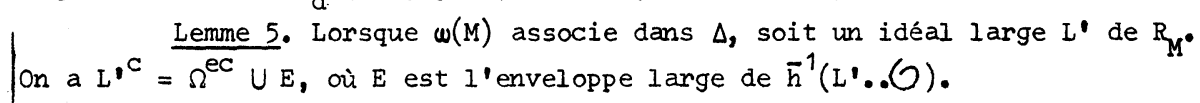

On peut se ramener au cas d'un annérde grâce au lemme 2 et à (III, $\S 4 b$, remarque 4); mais la preuve directe est aussi simple : pour tout $a \in \bar{h}^{-1}\left(L^{\prime} . . O\right)$, comme $h(a) \notin \mathcal{O}$ et $a \notin \Omega$, les grades $\omega(a)=\alpha$ et $\omega^{\prime}(h(a))=\frac{\sigma \alpha}{\sigma}$ (où I'on pose $\sigma=\omega(s) \in \omega(M)$ ) sont non nuls. Pour $\mu=\alpha$ ou pour tout multiple $\mu=\delta \alpha$ (avec $\delta \in \Delta$ ) non vide dans $R$, et pour tout élément $\mathrm{b} \in \mathrm{R}_{\mu} \ldots \Omega$ tel que $\mathrm{b} \notin \Omega^{\mathrm{ec}}$, on a $\omega^{\prime}(h(b))=\omega^{\prime}\left(\frac{s b}{s}\right)=\frac{\sigma \mu}{\sigma}=\frac{\sigma \delta}{\sigma} \frac{\sigma \alpha}{\sigma}$ ou $\frac{\sigma \alpha}{\sigma}$. Puisque L' est large en grade $\frac{\sigma \alpha}{\sigma}$, il $l^{\prime}$ 'est en grade $\frac{\sigma \mu}{\sigma}$ et $l^{\prime}$ on $a h(b) \in L^{\prime}$, soit $b \in L^{\prime c}$. Par suite, $L^{\prime c}$ contient $l$ 'enveloppe large de $a$, donc enfin celle de $\bar{h}^{-1}\left(L^{\prime} . . O\right)$.

Lemme 6. Si $L$ est idéal $\Delta-1$ arge de $I$ 'anel $R$, et si $\omega(M)$ associe dans

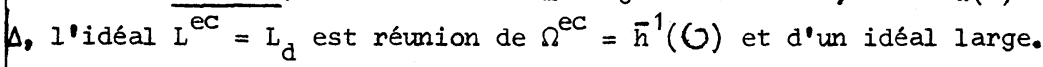

$L^{e}$ étant large dans $R_{M}$ (lemme 4 ), le lemme 5 montre le résultat.

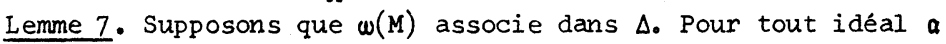
contenant $\Omega$ et tel que $M$ soit premier à $a$, le noyau large de $a^{e}$ est 1 'extension du noyau large de $a$.

a contient $a_{L}$, donc $a^{e}$ contient I'idéal $\left(a_{L}\right)^{e}$, qui est large (lemme 4). Tout $x \in a^{e}, x \notin\left(a_{L}\right)^{e}$, s'écrit $x=\frac{a}{s}$ (III, $\S 4 b$, lemme 6) avec $a \in a_{0} a_{L}$ vérifiant la condition : pour tout $m \in M$, ma $\notin a_{L}$. Puisque $a_{L}$ est le noyau large de $a$, il existe $b \in R_{0} . a$, dont le grade $\beta=\omega(b)$ est ou $\alpha=\omega(a)$ ou un multiple $\delta \alpha$. Puisque $M$ est premier à $a$, la fraction $\frac{b}{s} n$ 'est pas dans $a^{e}$ (III, $\S 4 b$, théorème 3 ), et son grade $\omega^{\prime}\left(\frac{b}{s}\right)=\frac{\beta}{\sigma}{ }^{5}$ est égal à $\frac{\alpha}{\sigma}$ ou $\frac{\delta \alpha}{\sigma}$, c'est-à-dire à un multiple du grade de $x$. Donc, $x n^{\prime}$ 'est pas dans le noyau large de $a^{e}$, et ce noyau large est $\left(a_{L}\right)^{e}$.

Lemme 8. Supposons que $\omega(M)$ associe dans $\Delta$, et que $p$ soit un idéal premier disjoint de M. Pour tout idéal p-primaire $q$ (et pour $p$ en particulier), on a : $\left(q^{e}\right)_{L}=\left(q_{L}\right)^{e}$. En particulier, si $q$ est étroit, son extension aussi.

En effet, $M$ est premier à $q$. 
Dans la suite, anels et anneldes sont supposés commutatifs, ainsi que leurs graduations éventuelles. $\S 1$ - ANELS ET MONELS NOETHERIENS.

Proposition et définition. Soit A un anel unitaire. Un A-monel unitaire $M$ sera dit noethérien si et seulement $s^{\prime} i l$ vérifie les conditions équivalentes suivantes :

1 - Toute suite croissante de sous-monels de $M$ est stationnaire.

2 - Toute famille non vide de sous-monels de $M$ possède un élément maximal pour l'inclusion.

3 - Tout sous-monel de $M$ est de type fini.

L'anel A sera dit noethérien $s^{\prime} i l$ l'est comme monel sur lui-meme.

La démonstration classique relative aux modules sur un anneau $s^{\prime}$ applique aux monels, sans changement. Remarquer que ces définitions comprennent le cas particulier d'un annérde A, et d'un A-modulorde M. D'autre part, les propriétés de noethériannité et de régularité sont étrangères : par exemple, un anneau non noethérien est un annérde régulier. Théorème 1 : Dans un anel noethérien, tout idéal contient une puissance de son radical, et tout idéal contient un produit d'idéaux premiers. En particulier, (0) est un produit d'idéaux premiers.

Soit $\left\{x_{1}, \ldots, x_{r}\right\}$ un système de générateurs de $\sqrt{a}$. Il existe un entier s tel que $x_{i}^{s} \in a$, pour tout $i=1, \ldots, r$. Soit $m=r(s-1)+1$. L'ensemble des éléments $y_{j}=\prod_{i=1}^{r} x_{i}^{\alpha_{i}}$ où $\alpha_{1}+\ldots+\alpha_{r}=m$ est un système de générateurs de $(\sqrt{a})^{m}$. Dans tout $y_{j}$, I'un au moins des exposants, soit $\alpha_{k}$ ' est au moins égal à s. Donc $x_{k}^{\alpha} \in a$, et on $a y_{j} \in a$ et $(\sqrt{a})^{m} \subset a$.

Soit F la famille des idéaux de $\mathrm{A}$ qui ne contiennent aucun produit d'idéaux premiers. Si $\mathrm{F}$ est non vide, elle possède un élément maximal $\mathfrak{J}$, puisque A est noethérien. Mais $\Im n^{\prime}$ 'est pas premier, et il existe deux idéaux $a$ et $b$, distincts de $\mathfrak{J}$, contenant $\Im$ et tels que $\mathbf{a} b \subset \boldsymbol{J}$. Puisque $\mathfrak{I}$ est maximal dans $F$, a (resp. b) contient un produit d'idéaux premiers. Il en est de meme de $\boldsymbol{a} \mathfrak{b}$, donc de $\boldsymbol{\Im}$, d'où une contradiction. 
Corollaire : Soit $\mathrm{A}$ un anel noethérien, et soit $q$ un idéal $p$-primaire. Il existe un entier $m \geqslant 1$ tel que $p^{m} \subset q ;$ le plus petit entier $m$ vérifiant cette relation est dit $l^{\prime}$ exposant de $q$. Si les idéaux $\boldsymbol{a}$ et $\boldsymbol{b}$ vérifient les conditions $\mathbf{a} b \subset \mathbf{q}$ et $\boldsymbol{a} \not \mathcal{q}$, il existe un entier $m \geqslant 1$ tel que $b^{m} \subset q$.

$q$ étant $p$-primaire, on $a b \subset p$ d'où $b^{m} \subset p^{m} \subset q$, si $m$ est $l^{\prime}$ exposant de $q$.

Théorème 2 : Si l'image d'un anel (resp. monel) noethérien par un quasi-morphisme est un anel (resp. monel), cette image est noethérienne.

Soit $u: A \rightarrow A_{1}$ un quasi-morphisme. On suppose que $u(A)$ est sous-anel de $A_{1}$. Soit $\left\{\mathfrak{h}_{h}\right\}$ une suite croissante d'idéaux de $u(A)$. $\left\{\bar{u}^{-1}\left(\mathfrak{H}_{n}\right)\right\}$ est une suite croissante d'idéaux de $\mathbf{A}$, donc elle est stationnaire. Comme $u \circ \vec{u}^{-1}\left(\mathfrak{f}_{n}\right)=\mathfrak{J}_{n}$, la suite $\left\{\mathfrak{I}_{n}\right\}$ est stationnaire. On le montre de même pour les monels. On en déduit :

Corollaire : L'image d'un annérde (resp. modulorde) noethérien par un homomorphisme est un annérde (resp. modulorde) noethérien.

Lemme 1 : Soient $M$ un monel sur un anel $A$, et $f$ un quasi-morphisme de source M. Si le A-monel agglutiné $\bar{M}^{f}$ est noethérien, alors $M$ est
noethérien.

Soient $O$ le milieu de $M, P$ et $Q$ deux sous-monels de $M$; notons $P^{\prime}$ et $Q^{\prime}$ les sous-monels engendrés dans $\bar{M}^{f}$ par $P$ et $Q$ respectivement. Supposons que $P^{\prime}=Q^{\prime}$, ce qui entraine $Q \subset P^{\prime}$. Tout élément $q \in Q$ est donc une somme finie d'éléments de $P$, non addibles dans $M$, mais addibles dans $\bar{M}^{f}$ et dans $\bar{M}: q=p_{1}+\ldots+p_{s}$. Mais $q \in M$, et $\bar{M}$ étant somme quasi-directe des groupes d'addibilité de $M$, les $p_{i}$ doivent appartenir à $O$, sauf un au plus. Par suite on a $S=1, q=P_{1}, P_{1} \in P$, et par conséquent $Q \subset P$; on $a \subset Q$ par symétrie. Donc $P^{\prime}=Q^{\prime}$ implique $P=Q$. Soit $\left\{P_{n}\right\}$ une suite croissante de sous-monels de $M$. La suite associée $\left\{P_{n}^{\prime}\right\}$ de sous-monels de $\bar{M}^{f}$ est croissante, donc stationnaire puisque $\bar{M}^{f}$ est noethérien. Ce qui précède montre que $\left\{P_{n}\right\}$ est stationnaire.

Théorème 3 : De deux A-monels (resp. anels) agglutinés comparables d'un même A-monel (resp. anel), si le plus agglutiné est noethérien, le moins agglutiné aussi est noethérien.

Deux agglutinés $P$ et $Q$ d'un même A-monel $M$ sont dits comparables $s^{\prime} i l s$ le sont comme parties de $\bar{M}$, c'est-à-dire ( $s i$ par exemple $P \subset Q$ ) si deux éléments addibles quelconques de $\mathrm{P}$ sont éléments addibles de $\mathrm{Q}$. On passe alors de $P$ à $Q$ par au plus deux agglutinations simples (II, $\S 3$ ). on en déduit le résultat par le lemme 1. Lorsqu'un agglutiné B d'un anel A 
est lui-même un anel, les notions de sous-A-monel et d'idéal de B se confondent. Ceci montre le théorème dans le cas des anels agglutinés de A.

Par contre, même si $u: A \rightarrow A_{1}$ (resp. $M \rightarrow M_{1}$ ) est un homomorphisme, $I^{\prime}$ image de $u$ peut $n^{\prime}$ être pas un sous-anel de $A_{1}$ (resp. un sous-nonel de $M_{1}$ ). De plus, l'addibilisé [Im $u$ ] de Im $u$ dans $A_{1}$ (resp. $M_{1}$ ), qui est le sous-anel (resp. le sous-monel) engendré par Im $u$, peut $n$ 'être pas noethérien (voir plus loin l'exemple contraire 3). En particulier, montrons qu'un anel A peut etre noethérien, sans que l'anneau associé $\bar{A}$ le soit.

Exemple contraire 1: Soit $Q$ le corporde des fractions monomiales sur le corps $\mathbf{C}$ des nombres complexes, par rapport à une infinité d'indéterminées $\left\{y_{i}\right\}_{i} \in \mathbb{N}$. Deux fractions sont addibles si elles sont semblables, ou si l'une est nulle. $Q$ est noethérien, mais $I^{\prime}$ anneau $\bar{Q}$ associé $n^{\prime}$ est pas

noethérien. Considérons en effet la suite croissante d'idéaux de $\bar{Q}$ définie par : $a_{0}=(0)$ et $a_{n+1}=a_{n}+\bar{Q}\left(y_{2 n}+y_{2 n+1}\right)$. Soit le $\mathbb{c}$-homomorphisme d'anneaux $\varphi: \bar{Q} \rightarrow \mathbf{C}$ défini par : pour tout $a \in \mathbf{C}, \varphi(a)=a ;$ pour tout $i<n$, $\varphi\left(y_{2 i+1}\right)=-1$; pour tout $i \leqslant n, \varphi\left(y_{2 i}\right)=\varphi\left(y_{2 n+1}\right)=1$. Alors on a $\varphi\left(a_{n}\right)=(0)$, mais $\varphi\left(a_{n+1}\right)=\mathbb{C}$; ceci montre que la suite des idéaux $a_{n}$ est strictement croissante, et que $\bar{Q} n^{\prime}$ est pas noethérien. D'autre part, considérons $\bar{Q}$ comme un anel égal à son milieu ; l'injection $j: Q \rightarrow \bar{Q}$ est un homomorphisme d'anels, et le sous-anel engendré par $j(Q)$ est $\bar{Q}$, non noethérien.

Théorème 4 : Soient $M$ un A-monel, et $N$ un sous-monel de $M$. Pour que $M$ soit noethérien, il faut et il suffit que $N$ et $M / N$ soient noethériens.

Si $M$ est noethérien, $\mathrm{N} I$ 'est aussı; le théorème 2 montre que $\mathrm{M} / \mathrm{N}$ est noethérien. Inversement, supposons $\mathrm{N}$ et $\mathrm{M} / \mathrm{N}$ noethériens. Soit $\mathcal{O}$ le milieu de $M$. Soit $\left\{\mathrm{P}_{n}\right\}$ une suite croissante de sous-monels de $M$. Posons $P_{n}^{\prime}=P_{n}+\mathcal{O}$ et $F_{n}^{\prime \prime}=P_{n} \cap \mathcal{O}$. Alors, $\left\{P_{n}^{\prime}\right\}$ et $\left\{P_{n}^{\prime \prime}\right\}$ sont des suites croissantes de sous-monels de $M_{0}\left\{P_{n}^{\prime} \cap N\right\}$ et $\left\{P_{n}^{\prime}+N / N\right\}$ sont des suites croissantes de sous-monels de $\mathrm{N}$ et de $\mathrm{M} / \mathrm{N}$ respectivement. Ces deux demières suites sont donc stationnaires à partir d'un entier $q$. Pour $n \geqslant q$. on a $P_{n}^{\prime} \cap N=P_{q}^{\prime} \cap N$ et $P_{n}^{\prime}+N / N=P_{q}^{\prime}+N / N^{\prime}$ d'où aussi $P_{n}^{\prime}+N=P_{q}^{\prime}+N$ (III, $\oint 1$, th. 4, bijection entre sous-monels). Le corollaire de la loi modulaire (II, $\S 2$ b) appliqué à $P_{n}^{\prime}, P_{q}^{\prime}, N$ montre que $P_{n}^{\prime}=P_{q}^{\prime}$. On montre de même que $P_{n}^{\prime \prime}=P_{q}^{\prime \prime}$. Le même corollaire appliqué à $P_{n}, P_{q}, U$ implique alors $P_{n}=P_{q}$, et la suite $\left\{P_{n}\right\}$ est stationnaire. Corollaire 1 : Soit $u: M \rightarrow M_{1}$ un homomorphisme de A-monels, vérifiant $[\mathrm{m}, \mathrm{M}]$. Si l'image et le noyau de $u$ sont noethériens, $M$ est noethérien.

En effet, I'image de $u$ est un sous-monel, et $u(M)$ et $M / K e r ~ u$ sont isomorphes (III, $\S 1$, th. 3 ). 
Corollaire 2 : Soit $u: M \rightarrow M_{1}$ un homomorphisme de moduloides. Si l'image et le noyau de $u$ sont noethériens, $M$ est noethérien.

Théorème 5 : Soit $M$ un A-monel de milieu 0 . Soient $\mathrm{M}_{1}, \ldots, \mathrm{M}_{\mathrm{S}}$ Les sous-monels tels que $M=M_{1}+\ldots+M_{s} \cdot(1)$ Pour que $M$ soit noethérien, 11 faut et il suffit que $M_{1}, \ldots, M_{s}$ et $\mathcal{O}$ soient noethériens.

(2) Si chaque $M_{i}$ est noethérien, et si tous les $M_{i}$, sauf un au plus, sont kontenus dans $\mathcal{O}, M$ est noethérien.

La condition (1) est évidemment nécessaire. D'autre part, si $M=N+P$, avec $N$ et $P$ noethériens, et si $N$ est comparable à $G$, nous savons (III, $\S 1$, th. 5) que $N+P / P$ est isomorphe à $N / N \cap P$, donc est noethérien (th. 4). Dans le cas (1) ou le cas (2), la démonstration s'achève par récurrence sur s. On a la conséquence immédiate :

Corollaire : Un A-monel, somme de sous-monels noethériens dont un contient le milieu, est noethérien.

Exemple contraire 2 : Montrons que la condition " $O$ noethérien" du théorème $5 \mathrm{n}^{\prime}$ est pas superflue. Soit A l'annérde des monômes

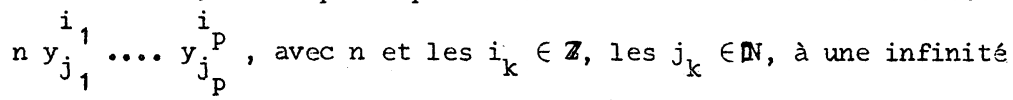
d'indéterminées. A est noethérien, ses seuls idéaux étant les idéaux monogènes $(n)$. Mais nous savons que $\bar{A} n$ 'est pas noethérien. Un support de la graduation propre $\Delta_{0}$ de $A$ est formé de 0 et des monômes de coefficient 1 . Construisons un $A$-monel $P$ de graduation $\Gamma=\Delta^{\prime} U \Delta^{\prime \prime}$, avec $\Delta^{\prime}$ et $\Delta^{\prime \prime}$ isomorphes à $\Delta_{0}$, et $\Delta^{\prime} \cap \Delta^{\prime \prime}=\{0\}$. Notons $\alpha^{\prime}$ (resp. $\alpha^{\prime \prime}$ ) I'homologue de $\alpha \in \Delta_{0}$ dans $\Delta^{\prime}$ (resp. $\Delta^{\prime \prime}$ ). Pour tout $\delta \in \Delta_{0}$, et pour tout $\alpha^{\prime}$ (resp. $\left.\alpha^{\prime \prime}\right) \in \Gamma$, on pose alors pour produit $\delta \alpha^{\prime}=(\delta \alpha)^{\prime}\left(\right.$ resp. $\delta \alpha^{\prime \prime}=\left(\begin{array}{l}\delta \\ \gamma\end{array}\right)^{\prime \prime}$.). Pour tout $\gamma \in \Gamma^{*}=\Gamma \ldots\{0\}$, on pose $P_{\gamma}=\bar{A} \times \bar{A}_{\text {. }}$ Le A-monel $P$ aura pour milieu $O=\bar{A}(1,1)$ contenu dans chaque $P_{\gamma}$, et sera égal à $\bigcup_{\gamma \in \Gamma}{ }^{*} P_{\gamma}$, avec le produit défini comme suit. Soient $\gamma \in \Gamma^{*},(u, v) \in \mathrm{P}_{\gamma}$ et $n \alpha \in A$; le produit de $n \alpha$ et de $(u, v)$ est le couple $(n \alpha u, n \alpha v) \in \mathrm{P}_{\alpha \gamma}$. Soit $\mathrm{K}$ le sous-monel engendré par les deux éléments $(3,0) \in \mathrm{P}_{1}$, et $(2,0) \in \mathrm{P}_{1}$ " K $\underline{K}$ est donc ainsi formé : pour $\alpha^{\prime} \in \Gamma$, on a $K_{\alpha^{\prime}}=(3 \mathbb{Z} \alpha, 0)$ et pour $\alpha^{\prime \prime} \in \Gamma$, on a $K_{\alpha^{\prime \prime}}=(2 \mathbf{z} \alpha, 0)$ (là interviennent les Iois produit sur $\Gamma$ et sur $P$ ). $X$ est noethérien, car tout sous-monel est engendré par deux éléments au plus : $(3 \mathrm{~m}, 0) \in \mathrm{P}_{1}$ ' et $(2 \mathrm{p}, 0) \in \mathrm{P}_{1 \text { " }}$ avec $m, p \in \mathbb{Z}$. Soit de même $\mathrm{L}$ le sous-monel noethérien de $P$ engendré par les leux éléments $(0,3) \in P_{1}$, $\in t(0,2) \in P_{1}$. $O$ et $K$ (resp. L) ont en commun le seul élément $(0,0)$. L'ólément $(1,1)$ qui engendre le milieu de $P$ est une combinaison linéaire des générateurs donnés pour $K$ et $L$ : $(1,1)=(3,3)-(2,2)=[(3,0)+(0,3)]-[(2,0)+(0,2)]$. On a donc 
$\mathcal{O} \subset K+L$, et $\mathcal{G}$ est un sous-A-monel non noethérien de $K+L$. Donc, la somme de deux sous-monels noethériens peut n'être pas noethérienne.

Exemple contraire 3 : Prenons $A, \Delta_{0}, \Gamma, P, K$ et $L$ comme dans $I^{\prime}$ exemple précédent. Soient $K^{\prime}$ et $L^{\prime}$ des $A$-monels (qui sont des moduloides) respectivement isomorphes à $K$ et $L$. Soit $M^{\prime}=K^{\prime} U L^{\prime}$ le $A$-monel dont le milieu est réduit à $(0,0)$. Soit enfin $u: M^{\prime} \rightarrow P^{\prime} I^{\prime h o m o m o r p h i s m e ~ a i n s i ~ d e ́ f i n i ~: ~}$ sa restriction à $K^{\prime}$ (resp. $L^{\prime}$ ) est le monomorphisme canonique $K^{\prime} \rightarrow K \rightarrow P$ (resp. $L^{\prime} \rightarrow L \rightarrow P$ ). Alors, $I^{\prime}$ image de $u$ est $X \cup L$, et $I^{\prime}$ agglutiné (dans $P$ ) de I'image de $u$ est $K+L$. On a donc un homomorphisme de A-monels dont la source $M$ ' est noethérienne, dont $I^{\prime}$ image $n^{\prime}$ 'est pas un sous-monel et dont $l^{\prime}$ agglutiné de $l^{\prime}$ image $n$ 'est pas noethérien.

Théorème 6 : Soit $A$ un anel unitaire. Pour que tout A-monel de type fini soit noethérien, il faut et il suffit que $\bar{A}$ soit un anneau noethérien.

Soit $M$ un A-monel, et soient $\bar{A}$ et $\bar{M}$ les anneau et module associés ; les notions de sous-A-monel et de sous $\bar{A}-$-module sont confondues pour $\bar{M}$. D'une part, $\bar{A}$ est $A-$ monel engendré par 1 ; il est donc nécessaire que $\bar{A}$ soit noethérien. Inversement, si $\bar{A}$ est un anneau noethérien, et si $M$ est un A-monel de type fini, $\bar{M}$ est de type fini comme A-monel, donc comme $\bar{A}-$ module. On sait alors que $\bar{M}$ est noethérien comme $\bar{A}$-module, donc comme $A-m o n e l$. Le théorème 3 montre que $M$ est un $A$-monel noethérien.

Théorème 4 bis : Soient $\mathrm{A}$ un anel, et $\mathfrak{Y}$ un idéal de A. (1) Si A est un anel noethérien, $A / \Im$ est un anel noethérien. (2) Si $\mathfrak{S}$ et $\mathrm{A} / \mathrm{S}$ sont des anels noethériens, A est un anel noethérien. La démonstration est analogue à celle du théorème 4 , et on en déduit de même :

Corollaire 1 bis : Soit $u: A \rightarrow A$, un homomorphisme d'anels vérifiant $[\mathrm{m}, \mathrm{A}]$. Si les anels $u(A)$ et Ker $u$ sont noethériens, $A$ est noethérien.

Corollaire 2 bis : Soit $u: A \rightarrow A$, un homomorphisme d'annérdes. Si les annérdes $u(A)$ et Ker $u$ sont noethériens, A est noethérien.

On appellera module (resp. anneau) quasi-gradué noethérien, un module (resp. anneau) dont les sous-modules (resp. idéaux) homogènes vérifient la condition maximale (nous dirons plus brièvement qu'il est homogènement noethérien). Si un module est noethérien, pour toute graduation, il est homogènement noethérien. Plus généralement, si un module (resp. anneau) est homogènement noethérien pour une graduation donnée, il l'est encore pour une graduation plus fine (c'est-à-dire restreignant l'ensemble des éléments homogènes). Par contre, un module quasi-gradué noethérien peut très bien n'être pas noethérien comme module. 
Dans un anneau quasi-gradué noethérien, tout idéal homogène contient une puissance de son radical homogène. Tout idéal homogène contient un produit d'idéaux premiers homogènes. En particulier, (0) est un produit d'idéaux premiers homogènes.

Soit un homomorphisme quasi-homogène d'un anneau quasi-gradué homogènement noethérien dans un autre anneau quasi-gradué. Si l'image de la partie homogène est additivement fermée dans la partie homogène du but, cette image est un anneau quasi-gradué homogènement noethérien. Une somme de sous-modules homogènement noethériens est homogènement noethérienne dans les cas suivants :

(1) l'un au moins des sous-modules contient le sous-module $\mathcal{O}$ de grade nul ; (2) tous les sous-modules, sauf un au plus, sont contenus dans 0 . Mais la somme de deux sous-modules homogènement noethériens peut ne pas l'etre, donc aussi n'etre pas un module noethérien.

Soient $\overline{\mathrm{M}}$ un $\overline{\mathrm{A}}$-module quasi-gradué, et $\overline{\mathrm{N}}$ un sous-module homogène de $\bar{M}$. Pour que $\bar{M}$ soit homogènement noethérien, il faut et il suffit que $\bar{N}$ et $\bar{M} /$ N le soient (outre le théorème 4 , voir III, $\S 1$, application). Indications sur les monels artiniens. Un monel $M$ sur un anel unitaire A sera dit artinien si et seulement s'il vérifie les conditions équivalentes suivantes : (1) toute suite décroissante de sous-monels de $M$ est stationnaire ; (2) toute famille non vide de sous-monels de $M$ possède un élément minimal pour l'inclusion. Un anel est dit artinien s'il l'est comme monel sur lui-même.

Les théorèmes $2,3,4,4$ bis, 5 et leurs corollaires sont encore vrais dans le cas artinien (les démonstrations sont analogues).

Si A est un anel artinien, I'anneau $\bar{A}$ peut n'etre pas artinien, et un A-monel monogène peut n'etre pas artinien. En effet, dans l'exemple contraire 1, on a la suite strictement décroissante d'idéaux (resp. de sous-Q-monels) de $\bar{Q}$ définie par $b_{n}=\left(\left(y_{1}+y_{2}\right)^{n}\right)$. $\$ 2$ - LE THEOREME DE HILBERT.

Rappelons que M. Krasner a déjà étudié [4] de manière très complète les annérdes de polynomes à $p$ variables sur un corporde $q$ sans torsion, (c'est-a-dire dont $\Delta^{*}=\Delta . .\{0\}$ est un groupe sans torsion, $\Delta$ étant la graduation propre de q). Indiquons d'abord un résultat immédiat, mais important.

Théorème 1 : Pour que tout anel de polynômes à une variable sur um anel A soit noethérien, il faut et il suffit que l'anneau $\bar{A}$ soit noethérien. La condition est suffisante, $\operatorname{car} \bar{A}[X]$ est alors noetherien, et est I'anneau agglutiné de tout anel $A_{E}[\mathrm{X}]$ de polynômes à une variable sur $A_{\text {. }}$ Cette condition est nécessaire pour le cas particulier $\xi=0$. 
Soient $A$ un anel de milieu $\Omega$, et de graduation donnée $\Delta$, un grade donné $\xi \in \Delta$, et enfin $A_{\xi}[X] 1$ 'anel des polynômes à une variable, de pente $\xi$, et $O$ le milieu de cet anel. Pour un idéal quelconque a de $A_{\xi}[x]$, on note $a^{\prime}=a+\mathcal{O}$ et $a^{\prime \prime}=a \cap \mathcal{O}$. On note $L_{i}(a)$ (resp. $M_{i}(a)$ ) la partie de $\bar{A}^{\xi, i}$ formée des coefficients dominants des polynômes de degré $i \geqslant 0$ de l'idéal $a^{\prime}$ (resp. a"). Nous avons les inclusions ensemblistes $L_{i}(a) \subset L_{i+1}(a)$ et $M_{i}(a) \subset M_{i+1}(a)$, car si $f \in a$ ou $\mathcal{O}, X f \in a$ ou $\mathcal{O}$. $L_{i}(a)$ et $M_{i}$ (a) sont des sous-A-monels de $\bar{A}^{\xi, i}$ : pour tout $a \in A, f \in a$ (resp. $O$ ) implique af $\in a$ (resp. $O$ ). Soient $\alpha$ et $\beta \in L_{i}(a)$; donc il existe des $\xi$-polymónes $f$ et $g \in a^{\prime}$ tels que $f=\alpha \mathrm{x}^{i}+\ldots$ et $\mathrm{g}=\beta \mathrm{x}^{i}+\ldots$. Si $\alpha$ et $\beta \mathrm{n}^{\prime}$ appartiennent pas au milieu de $\vec{A}^{\xi, i}$, $f$ et $g$ sont addibles, donc $f-g \in a^{\prime}$ et $\alpha-\beta$ est dans $L_{i}(a)$. Si par exemple $\beta$ est dans le milieu de $\bar{A}^{\xi}, i$, alors $\beta x^{i} \in \mathcal{O}$, on a donc $f-\beta x^{i} \in \Theta$, d'où encore $\alpha-\beta$ est dans $L_{i}(a)$. Donc $L_{i}(a)$ est un sous-A-monel de $\bar{A}^{\xi, i}$. La démonstration analogue pour $M_{i}(a)$ est plus simple, car deux polynomes de $a^{\prime \prime}$ sont toujours addibles.

Si de plus $\xi$ associe, $\bar{A}^{\xi, i}$ est un anel, et $L_{i}(a)$ et $M_{i}(a)$ sont des idéaux de cet anel.

Lemme 1 : Soient $a$ et $b$ dewx idéaux de $A_{\xi}[x]$ vérifiant les conditions suivantes : $a \subset b$; pour tout entier $i \geqslant 0$, on $a L_{i}(a)=L_{i}$ (b) et $M_{i}(a)=M_{i}(b)$. Alors, $a$ et $b$ sont égaux.

Soit $g=\beta x^{i}+\ldots$ un polynome de $b \subset b^{\prime}=b+\mathcal{O}$. si $\beta x^{i}$ appartient à $O$, donc à $a^{\prime}$, on prend $f_{i}=\beta x_{i}^{i}$. Sinon, puisque $L_{i}(a)=L_{i}(b)$, il existe un polynome $f_{i} \in a^{\prime}$ de la forme $\beta x^{i}+\ldots$; $g$ et $f_{i}$ sont addibles, car ils le sont à $\beta x^{i} \notin^{\prime} \mathcal{O}$. Dans les deux cas, comme $a^{\prime} \subset b^{\prime}, g-f_{i}$ appartient à $b^{\prime}$ et son degré est $\leqslant i-1$. Par récurrence sur $j$, on obtient ainsi une suite $\left\{f_{i-j}\right\}(j=1,2, \ldots)$, où $f_{i-j} \in a^{\prime}$ est nul ou de degré $i . j$, où $g$ et les $f_{i-j}$ sont addibles entre eux, suite telle que $g-f_{i}-\ldots-f_{i-j}$ soit un élément de $b^{\prime}$ nul ou de degré $\leqslant i-j-1$. Pour $j=i$, la différence sera nulle, d'où $g \in a^{\prime}=a+\mathcal{U}$. Par suite, il existe $f \in a$ et $\varphi \in \mathcal{O}$ tels que $g=f+\varphi, d^{\prime}$ où $\varphi=g-f \in a+b \subset b$, et $\varphi \in b^{\prime \prime}$. Soit $\varphi=\gamma x^{i}+\ldots$; puisque $M_{i}(a)=M_{i}(b)$, il existe un élément $h_{i} \in a^{\prime \prime}=a \cap \mathcal{O}$ de la forme $n_{i}=x^{i}+\ldots$. Dans $O$ tout est addible, et $\varphi-h_{i}$ est un élément de $b^{\prime \prime}$ nul ou de degré $\leqslant i-1$. Par récurrence sur $j$, on obtient une suite $\left\{h_{i-j}\right\}(j=1,2, \ldots)$ où $h_{i-j} \in a^{\prime \prime}$ est nul ou de degré $i-j$, où $\varphi-h_{i}-\ldots-h_{i-j} \in$ b" est nul ou de degré $\leqslant i-j-1$. Pour $j=i$, la différence est nulle et $\varphi \in a^{\prime \prime} \subset a$. Finalement, $g$ est dans $a$, et $a=b$.

Montrons qu'un anel de polynomes sur un anel noetherien fort $n$ 'est pas forcément noethérien. Dans l'exemple suivant, tous les produits sont 
commutatifs, et on note $\mathbb{N}$ l'ensemble des entiers naturels et $\mathbf{c}$ le corps des nombres complexes. On considère les indéterminées $x$ et $\left\{y_{i}\right\}_{i} \in \mathbb{N}$ et le monorde $\Delta$ formé de l'annulateur 0 , et des monômes ( 1 compris) par rapport à $x, y_{i}$ et $y_{i}^{-1}\left(i \in \mathbb{N}\right.$ ) avec les relations $x^{s}=x^{s+1}$ ( $s$ entier $\geqslant 1$ ) et, pour un entier $d$ donné, $1 \leqslant \mathrm{~d} \leqslant \mathrm{~s}$, quels que soient $\alpha \in \mathbb{Z}$ et $i \in \mathbb{N}$, $y_{i}^{\alpha} x^{d}=x^{d}$. Soit A l'annérde construit sur $\Delta$ et $\operatorname{sur} \boldsymbol{c}$ (voir chap. I, $\S 2$, exemple $3^{\circ}$ et prendre $R_{\alpha}=\mathbb{C}$ ). Le corpoide $\mathrm{Q}$ des fractions monomiales par rapport aux $y_{i}$ est un sous-annérde unitaire de $A$. A est un annérde noethérien fort (ses seuls idéaux sont $(0)$ et les $\left(x^{j}\right)_{0}$ ), de graduation $\Delta_{\text {. Posons }}$ $\boldsymbol{\xi}=\boldsymbol{\omega}(\mathrm{x})$. L'anel de polynômes $\mathrm{A}_{\boldsymbol{\xi}}[\mathrm{X}]=\mathrm{R}$ est un annérde fort (clair), mais $n^{\prime}$ est pas noethérien. Considérons la suite d'idéaux définis par $J_{0}=(0)$ et $J_{n+1}=J_{n}+R \cdot\left(y_{2 n}+y_{2 n+1}\right) x^{d}$. Soit $p_{n}$ la partie de $J_{n}$ formée des polynômes de degré total d par rapport à $x$ et $x ; x n^{\prime} y$ figure pas, puisque $x^{d}$ est en facteur dans tout élément de $J_{n}$. Donc $P_{n} s^{\prime o b t i e n t ~ e n ~ m u l t i p l i a n t ~ p a r ~} x^{d}$ l'ensemble $a_{n}$ déterminé par $a_{0}=(0)$ et $a_{n+1}=a_{n}+\bar{Q}_{0}\left(y_{2 n}+y_{2 n+1}\right)$. La suite $\left\{a_{n}\right\}$ est une suite strictement croissante d'idéaux de $\bar{Q}$ (voir $\S 1$, exemple contraire 1). Donc la suite $\left\{J_{n}\right\}$ est strictement croissante et $A_{\xi}[\mathrm{X}] \mathrm{n}$ 'est pas noethérien.

Théorème 2 (de Hilbert) : Soient A un anel de graduation $\Delta$, et $\boldsymbol{\xi} \in \Delta$. Supposons qu'il existe un entier $r \geqslant i$ tel que pour tout $i \geq r$, on ait $\bar{A}^{\xi, i}=\bar{A}^{\xi, r}$. Si le $A-m o n e l \bar{A}^{\xi, r}$ est noethérien, 1 anel de polynomes $A_{\xi}[\mathrm{X}]$ est noethérien.

Faisons deux remarques. D'abord, lorsqu'une puissance de $\xi$ n'est pas un produit associatif, ou lorsque $\xi$ est nilpotent, I'hypothèse signifie que $\overline{\mathbf{A}}$ est noethérien. Ensuite si, outre les hypothèses du théorème, $\xi$ associe dans $\Delta(I I, \S 3$, lemme 4$)$, pour tout $i \geqslant 1, \bar{A}^{\xi, i}=\bar{A}^{i}$ est un anel, et 1 'hypothèse noethérienne porte en fait sur 1 'anel $\bar{A}^{\xi^{r}}$ (pour ce dernier, les notions d'idéal et de sous-A-monel sont confondues.).

Remarquer que les hypothèses impliquent A noethérien. Soit $\left\{a_{j}\right\}_{j=0,1, \ldots}$ une suite croissante didéaux de $A_{\xi}[X]$. La suite double $L_{i}\left(a_{j}\right)$ croit avec $i$ (resp. $j$ ) lorsque $j$ (resp.i) est fixé, et il en est de même de la suite double $M_{i}\left(a_{j}\right)$. $\xi$ étant quasi-régulier pour $r$, quels que soient $i \geqslant r$ et $j \geq 0, L_{i}\left(a_{j}\right)$ est un sous-A-monel de $\bar{A}^{\xi}, r$. Ce dernier étant noethérien, la famille $\left\{L_{i}\left(a_{j}\right)\right\}_{i} \supseteq r, j \geqslant 0$ possède un élément maximal, soit $L_{p}\left(a_{q}\right)$ (avec $p \geqslant r$ ). Alors, pour tout $i \geqslant p$, on a pour tout $j \geqslant q, L_{p}\left(a_{q}\right)=L_{i}\left(a_{q}\right)=L_{i}\left(a_{j}\right)$. $D^{\prime}$ autre part, pour tout $i<p$, on sait que $\bar{A}^{\xi}, i$ est noetherien, puisque $\bar{A}^{\xi}, r$, 
qui est un agglutiné, est noethérien. Donc, quel que soit $i<p$, il existe $j_{0}(i)$ tel que la suite $\left\{L_{i}\left(a_{j}\right)\right\}_{j=0,1, \ldots}$ soit stationnaire pour $j \geqslant j_{0}(i)$. Posons $1=\sup \left[p,\left\{j_{0}(i)\right\}_{i<p}\right]$. Alors $j \geqslant I$ implique $L_{i}\left(a_{j}\right)=L_{i}\left(a_{1}\right)$ pour tout $i$. Ce qui précède s'applique sans changement à $M_{i}\left(a_{j}\right)$, et il existe un entier $m \geqslant r$ tel que, pour tout $i, j \geqslant m$ implique $M_{i}\left(a_{j}\right)=M_{i}\left(a_{m}\right)$. Posons $k=\sup (1, m)$. Alors, quel que soit $j \geqslant k$, les idéaux $a_{k}$ et $a_{j}$ vérifient les conditions du lemme, donc sont égaux. La suite des idéaux $a_{j}$ est donc stationnaire.

Définition 1 : Soient $\Delta$ une graduation, et $\xi \in \Delta$. Si les puissances de $\xi$ sont associatives, $\xi$ sera dit quasi-régulier (dans $\Delta$ ) s'il existe un indice $r=r(\xi) \geqslant 1$ tel que, pour tout $n \in \mathbb{N}$ et pour tous $\alpha, \beta \in \Delta$, la relation $\alpha \xi^{n}=\beta \xi^{n}$ implique $\alpha \xi^{r}=\beta \xi^{r}$. Une graduation $\Delta$ sera dite forte si tout $\xi \in \Delta$, dont les puissances sont associatives, est quasi-régulier.

Définition 2 : Soient $\Delta$ une graduation, et $\xi \in \Delta . \xi$ sera dit stationnaire (dans $\Delta$ ) s'il existe un indice $r=r(\xi) \geqslant 1$ tel que, pour tout $n \geq 1:\left(1^{\circ}\right)$ quel que soit le produit non associatif $\omega_{1} \ldots \omega_{s} \xi \ldots \xi$ (les $\omega_{j} \in \Delta ; n$ facteurs $\xi$ ), le produit $\omega_{1} \ldots \omega_{s} \xi \ldots \xi$ (r facteurs $\xi$ ) est aussi non associatif ;

$\left(2^{\circ}\right)$ si les puissances de $\xi$ sont associatives, $\xi$ est quasi-régulier pour $r$. La graduation $\Delta$ sera dite très forte si tout $\xi \in \Delta$ est stationnaire.

Corollaire 1 : Soient $A$ un anel de graduation $\Delta$, et $\xi \in \Delta_{0}$ Si $\xi$ est stationnaire pour $I^{\prime}$ indice $r$, et si $\bar{A}^{\xi, r}$ est noethérien, $A_{\xi}[\mathrm{X}]$ est noethérien.

Soit un entier $\mathrm{e} \geq \mathrm{r}$. Si, pour toute factorisation de $a \in A . . \Omega$, $a=\prod_{j=1}^{q} f_{j}$, le produit $\omega\left(f_{1}\right) \ldots \omega\left(f_{q}\right) \xi \ldots \xi$, avec e facteurs $\xi$, est associatif, on a $\omega_{e}^{\prime}(a)=\omega(a) \xi^{e}$ ( $\Delta$-grade $\omega^{\prime}$ vu en II, $\S 3$ d), et il en est de même du produit avec $r$ facteurs $\xi_{0}$. Si $a, b \in A_{0} . \Omega$ vérifient ces conditions pour I'indice e, avec $\omega(a) \xi^{e}=\omega(b) \xi^{e}$, puisque $\xi$ est quasi-régulier, on a $\omega(a) \xi^{r}=\omega(b) \xi^{r}$. Si, pour une factorisation de a $\in A_{0} . \Omega$, $a=\prod_{j=1}^{q} f_{j}$, le produit $\omega\left(f_{1}\right) \ldots \omega\left(f_{q}\right) \xi \ldots \xi$, avec e facteurs $\xi$, n'est pas associatif (donc $\omega_{e}^{\prime}(a)=0$ ), il en est de même, puisque $\xi$ est stationnaire, du produit avec $r$ facteurs, et l'on a $\omega_{r}^{\prime}(a)=0$. Notons le cas particulier suivant :

Corollaire 2 : Soient $A$ un anel de graduation $\Delta$, et $\xi \in \Delta$. Si $\xi$ associe dans $\Delta, s^{\prime} i l$ est quasi-régulier pour $r$, et si $\bar{A}^{\xi}, r$ est noethérien, alors $A_{\xi}[X]$ est noethérien. 
Lemme 2 : Soient $\mathrm{A}$ un anel de graduation $\Delta, \xi \in \Delta$ et $\mathrm{b} \in \mathrm{A}$. Si $\xi$ et $\omega(\mathrm{b})$ associent dans $\Delta$, et si $\xi$ divise $\omega(\mathrm{b})$, chacun des anels suivants est un agglutiné du précédent :

$$
\bar{A}^{\xi}, e, \bar{A}^{\omega(b)}, e, \bar{A}^{b^{e}}, \Lambda^{b^{e}} \text {. }
$$

Pour ces quatre agglutinés de $\mathrm{f}$, les notations sont définies

en II, $\S 3$. Posons $\beta=\omega(b)$ et $\beta=\xi \eta$. Comme $\xi$ et $\beta$ associent, les $\Delta$-grades de $a \in A$ dans $\bar{A}^{\xi}, e$ et dans $\bar{A}^{\beta, e}$ sont respectivement $\omega^{\prime}(a)=\omega(a) \xi^{e}$ et $\omega^{\prime \prime}(a)=\omega(a) \beta^{e}$. Supposons que $l^{\prime}$ on ait $\beta^{e}=\left(\xi^{n} \beta^{e-n}\right) \lambda$, avec $\lambda \in \Delta$, pour un entier $n, 0 \leqslant n<e$. On utilise successivement l'associativité par $\beta, \xi, \xi, \xi, \beta, \xi$ pour écrire : $\beta^{e}=\left\{\left[\xi^{n}(\xi \eta)\right] \beta^{e-n-1}\right\} \lambda=\left[\left(\xi^{n+1} \eta\right) \beta^{e-n-1}\right] \lambda=$ $=\left[\xi^{n+1}\left(\beta^{e-n-1} \eta\right)\right] \lambda=\xi^{n+1}\left[\left(\beta^{e-n-1} \eta\right) \lambda\right]=$ $=\xi^{n+1}\left[\beta^{e-n-1}(\eta \lambda)\right]=\left(\xi^{n+1} \beta^{e-n-1}\right)(\eta \lambda)$. Ceci montre par récurrence l'existence de $\mu \in \Delta$ tel que $\beta^{e}=\xi^{e} \mu$. Puisque $\xi$ associe, on en déduit : $\omega^{\prime \prime}(a)=\omega(a)\left(\xi^{e} \mu\right)=\left(\omega(a) \xi^{e}\right) \mu=\omega^{\prime}(a) \mu$. Par suite, tout agglutiné par $\xi, e$ l'est aussi par $\beta, e$, et d'autre part le milieu de $\bar{A}^{\beta, e}$ contient celui de $\bar{A}^{\xi}$, e Finalement, $\vec{A}(b), e$ est un agglutiné de $\bar{A}^{\xi}, e$. Soit $\Omega$ le milieu de $A$. Si $b^{e} \in \Omega$, cn a $\bar{A}^{e}=\bar{A}$. Supposons maintenant $b^{e} \notin \Omega$, ce qui entraine $\omega\left(b^{e}\right)=\beta^{e}$. Soit alors $\alpha=\sum_{i} a_{i} \in \bar{A}^{\beta, e}$. Les $w\left(a_{i} b^{e}\right)$ non nuls ont pour valeur $\omega\left(a_{i}\right) \beta^{e}$, donc sont égaux puisque $\alpha \in \bar{A}^{\beta, e}$. Si de plus $\alpha$ est dans le milieu de $\bar{A}^{\beta}, e$, j.l est donc dans le milieu de $\overline{\mathrm{A}}^{\mathrm{b}}$. Donc $\overline{\mathrm{A}}^{\mathrm{b}}$ est un agglutiné de $\overline{\mathrm{A}}^{-\beta, e}$. La troisième relation d'agglutination est connue.

Lemme 3 : Soient $\mathrm{A}$ un anel de milieu $\Omega, \mathrm{b} \in \mathrm{A}$ et $\mathrm{i}$ un entier $\geq 1$. Notons $k_{i}$ le noyau de $I$ 'homomorphisme $u_{i}: \Lambda^{b^{i}} \rightarrow A$ de A-monels défini par $\alpha \mapsto \alpha b^{i}$. Si $k_{1}$ est un A-monel (ou un $\bar{A}$-module) noetherien, il en est de méme de $k_{i}$ pour tout $i$ 。

Pour chaque $i$, par définition de $\Lambda^{b^{i}}$, $v_{i}$ est un homomorphisme de A-monels, et $k_{i}$ est contenu dans le milieu. $\circlearrowleft_{j}$ de $\Lambda^{b^{i}}$. Donc $k_{i}$ est son propre agglutiné dans $I^{\prime}$ anneau $\bar{A}$, et le $A-m o n e l ~ k_{i}$ peut être considéré comme un $\bar{A}$-module (donc comme un idéal de $\bar{A}$ ) ; pour $k_{i}$, les notions de sous-A-monel et de sous $\bar{A}$-module sont confondues. Montrons le lemme par récurrence sur $i$. Notons $\varphi$ I'homothétie de $\bar{A}$, de rapport $b^{i}$, qui est un homomorphisme de $\bar{A}$-modules. Pour $\alpha \in \bar{A}$ les conditions suivantes sont équivalentes : $\alpha \in k_{i+1}$, $\alpha b^{i+1}=0, \alpha b^{i} \in k_{1}$. Donc $\varphi$ se restreint en un homomorphisme $\psi: k_{i+1} \rightarrow k_{1}$ 
de $\bar{A}$-modules. Son noyau est ker $\downarrow=\left\{\alpha \in k_{i+1} ; \alpha b^{i}=0\right\}=\left\{\alpha \in k_{i+1} ; \alpha \in k_{i}\right\}=k_{i}$. Si $k_{1}$ et $k_{i}$ sont des $\bar{A}$-modules noethériens, il en est donc de même du noyau et de $I$ 'image de $\downarrow$, et $I$ 'on sait alors que $k_{i+1}$ est un $\bar{A}$-module noethérien.

D'autre part, $l$ 'hypothèse que $k$, soit un anneau noethérien est suffisante pour le lemme.

Théorème 3 : Soient $A$ un anel de graduation $\Delta, \xi \in \Delta$ et $\mathrm{b} \in \mathrm{A}$. Soit $k_{1}$ le noyau de $l$ 'homomorphisme $u_{1}: \Lambda^{b} \rightarrow$ A défini par $u_{1}(\alpha)=\alpha b$. on suppose que $\xi$ et $\omega(t)$ associent dans $\Delta$, que $\xi$ divise $\omega(b)$, et que $\xi$ est quasi-régulier pour l'indice $r_{0}$ Si les A-monels $A$ et $k_{1}$ sont noethériens, l'anel de polynomes $A_{\xi}[X]$ est noethérien.

$$
\text { L'homomorphisme } u_{r}: \Lambda^{b^{r}} \rightarrow \text { A défini par } u_{r}(\alpha)=\alpha b^{r} \text {, vérifie }
$$
$\left[\mathrm{m}, \Lambda^{\mathrm{b}^{\mathrm{r}}}\right]$ par construction de I'agglutiné large $\Lambda^{\mathrm{b}^{r}}$. Son noyau Ker $u_{r}$ est noethérien puisque $k_{1}$ l'est, d'après le lemme 3 . Par suite, $\Lambda^{b^{r}}$ est un A-monel noethérien (IV, $\S 1$, corollaire 1 du théorème 4), puisque A est noethérien. Mais (lemme 2), $\Lambda^{b^{r}}$ est un agglutiné de $\bar{A}^{\xi}, r$; il en résulte (IV, $\S 1$ th. 3 ) que $\bar{A}^{\overline{5}}, \mathrm{r}$ est noethérien. Donc, grâce au théorème 2,1 'anel de polynômes est noethérien.

Soit $A$ un anel de graduation $\Delta$ et de milieu $\Omega$. On rappelle (III, $\S 2$ d) que deux éléments $\gamma, \delta \in \Delta$ sont dits agglutinés par $\alpha \in \Delta$ si $\alpha \gamma=\alpha$ 8. Pour tout $a \in A$, posons $N(a)=\{x \in A ; a x \in \Omega\}=\Omega:\{a\}$. Si h est 1 'homothétie de $A$, de rapport $a, N(a)=\bar{h}^{-1}(\Omega)$ est donc un sous-A-monel de $A$, donc un idéal.

Lemme 4: Soient $A$ un anel de graduation $\Delta$, et $a, b \in A$. Si $\alpha=\omega(a)$ et $\beta=\omega(b)$ produisent une même agglutination dans $\Delta$, et si $N(a)=N(b)$, les agglutinés $\bar{A}^{a}$ et $\bar{A}^{b}$ sont égaux.

$$
N(a)=N(b) \text { signifie que pour tout } x \in A \text {, ax } \in \Omega \text { équivaut à }
$$

$\mathrm{bx} \in \Omega_{0}$. Lorsque $\omega(\mathrm{ax}) \neq 0$ (ce qui équivaut donc à $\omega(\mathrm{bx}) \neq 0$ ), comme $\alpha$ et $\beta$ agglutinent de meme dans $\Delta$, on a : $\omega(a x)=\omega(a) \omega(x)=\alpha \omega(x)=\beta \omega(x)=\omega(b) \omega(x)=\omega(b x)$. Soit alors $\xi=\sum_{i} x_{i} \in \bar{A}^{a}$. Les $\omega\left(a x_{i}\right) \neq 0$ ont donc une même valeur $\gamma$. Ce qui précède montre que $\omega\left(a x_{i}\right)=0$ implique $\omega\left(b x_{i}\right)=0$, et que $\omega\left(a x_{i}\right)=\gamma$ implique $\omega\left(b x_{i}\right)=\gamma$. Donc les $\omega\left(b x_{i}\right) \neq 0$ ont une même valeur, par suite $\xi \in \bar{A}^{b}$, et le milieu de $\bar{A}^{a}$ est contenu dans celui de $\bar{A}^{b}$. Donc $\bar{A}^{b}$ est un agglutiné de $\bar{A}^{a}$. Par symétrie, ces deux agglutinés sont égaux. 
Théorème 4 : Dans un anel noethérien $A$ dant une graduation est forte, pour tout a $\in A$, la suite des agglutinés $\bar{A}^{n}(n=1,2, \ldots)$ est stationnaire, et $A$ est un anel fort.

Soient A l'anel noethérien, $\Omega$ son milieu, et $\Delta$ sa graduation forte. Soit a $\in$ A. S'il existe une puissance $m(a) \geqslant 1$ de a, qui soit dans $\Omega$ (on dira, dans ce cas, que a est nilpotent par rapport au milieu $\Omega$ de $A$ ), pour tout $n \geq m(a), a^{n}$ appartient à $\Omega$, donc agglutine complètement $A$ en $\bar{A}_{0}$ La suite des agglutinés $\overline{\mathrm{A}}^{\mathrm{n}}, \mathrm{n}=1,2,3, \ldots$ est donc stationnaire à partir de $\mathrm{m}(\mathrm{a})$. Donc a est quasi-régulier.

Si a n'est pas nilpotent par rapport à $\Omega$, pour tout entier $n \geq 1$, $a^{n} n^{\prime}$ est pas dans $\Omega$, donc $\omega\left(a^{n}\right) \neq 0$, et le produit de $n$ facteurs $\alpha=\omega(a)$ est associatif. Puisque $\Delta$ est forte, il existe un entier $r(\alpha)$ tel que, pour tout $n \geq r(\alpha), \alpha^{n}$ produise la même agglutination que $\alpha^{r(\alpha)}$ dans $\Delta$. D'autre part, puisque A est noethérien, la suite d'idéaux $N\left(a^{n}\right), n=1,2, \ldots$ est stationnaire à partir d'un entier $s(a)$. Posons $t(a)=\sup (r(a), s(a))$. Pour tout $n \geq t(a), \alpha^{n}$ et $\alpha^{t(a)}$ produisent la nềme agglutination dans $\Delta$, et $\mathrm{N}\left(\mathrm{a}^{\mathrm{n}}\right)=\mathrm{N}\left(\mathrm{a}^{\mathrm{t}(\mathrm{a})}\right)$. Le lemme 4 montre que les agglutinés $\bar{A}^{\mathrm{t}(\mathrm{a})}$ et $\overline{\mathrm{A}}^{\mathrm{a}}$ sont égaux. Donc a est quasi-régulier.

Théorème 5: Soient $\mathrm{A}$ un annérde noethérien de graduation $\Delta$, $\gamma \in \Delta$ et $c_{1}, \ldots, c_{s}$ des éléments non nuls de grade $\gamma$. Pour chaque $i=1, \ldots, s$, soit $\varphi_{i}: \bar{A}^{\gamma} \rightarrow A$ I'homomorphisme de A-monels défini par $\varphi_{i}(\alpha)=\alpha c_{i} \cdot \operatorname{si} \bigcap_{i=1}^{S} \operatorname{ker} \varphi_{i}$ est un A-monel noethérien, alors $\bar{A}^{\gamma}$ est un A-monel noethérien.

D'abord, il est clair que $\Lambda^{C^{i}}$ est un agglutiné de $\bar{A}^{\gamma}$. Soit $u_{i}: \Lambda^{c_{i}} \rightarrow A$ I'homomorphisme de A-monels défini par $u_{i}(\alpha)=\alpha c_{i}$. $\varphi_{i}$ est la restriction de $u_{i}$ à $\vec{A}^{\gamma}$, donc est un homomorphisme. De plus $\varphi_{i}$ vérifie [m, $\bar{A}^{\gamma}$ ] trivialement, puisque A est un annélde. Donc, l'image de $\varphi_{i}$ est un idéal de A, donc un A-modulorde noethérien.

Notons $\mathrm{R}$ la partie du produit cartésien $\mathrm{A}^{\mathrm{S}}$ formée des s-uples $\left(a_{1}, \ldots, a_{s}\right)$ où les $a_{i}$ sont deux à deux addibles. Deux éléments $\left(a_{1}, \ldots, a_{s}\right)$ et $\left(b_{1}, \ldots, b_{s}\right)$ de $R$ seront addibles si et seulement si les $a_{i}$ et $b_{j}$ sont deux à deux addibles; alors, leur somme se fait par composantes. De même, le produit par $\lambda \in A$ d'un élément de $R$ se fait par composantes. Ainsi, $R$ est un A-modulorde, comme on peut le voir aisement.

$$
\text { Pour tout } \alpha=\sum_{j=1}^{n} a_{j} \in \bar{A}^{\gamma} \text {, les } a_{j} c_{i} \neq 0 \text { sont tous addibles entre }
$$


eux, par définition de $\bar{A}^{\gamma}$. Considérons donc $l^{\prime}$ application $\varphi: \bar{A}^{\gamma} \rightarrow \mathrm{R}$ définie par $\varphi(\alpha)=\left(\alpha c_{1}, \ldots, \alpha c_{s}\right), \varphi$ est un homomorphisme de A-monels vérifiant $\left[\mathrm{m}, \overline{\mathrm{A}}^{\gamma}\right]$, puisqu'il en est de même de chaque composante $\varphi_{i}$. $D^{\prime}$ après le corollaire 1 du theorème $4\left(\mathrm{IV}, \S 1\right.$ ), comme R est noethérien, pour que $\bar{A}^{\gamma}$ soit noethérien, il suffit que le noyau de $\varphi$ le soit. Or, ce noyau est $\operatorname{Ker} \varphi=\left\{\alpha \in \overline{\mathrm{A}} ; \forall_{i}, \alpha c_{i}=0\right\}=\bigcap_{i=1}^{s} \operatorname{ker} \varphi_{i}$.

Application : Soient $A$ un annérde noethérien de graduation $\Delta$, et $\xi, \beta \in \Delta$. Supposons que $\xi$ divise $\beta$, que $\xi$ et $\beta$ associent dans $\Delta$, et que $\xi$ soit quasi-régulier pour $r$.

$1 \%$ L'idéal engendré par le groupe d'addibilité A $\gamma\left(\right.$ avec $\gamma=\beta^{r}$ ) possède un système fini $\left\{c_{1}, \ldots, c_{s}\right\}$ de générateurs pris dans $A_{\gamma} \cdot$ si de plus (avec les notations du théorème 5), le A-monel $\bigcap_{i}$ ker $\varphi_{i}$ est noethérien, l'anel $A_{\xi}[X]$ est noethérien.

$2 \%$ L'idéal $(\beta)$ de $A$ engendré par le groupe d'addibilité $A_{\beta}$ possède un système fini $\left\{b_{1}, \ldots, b_{t}\right\}$ de générateurs pris dans $A_{\beta}$. Les produits $c_{i}$ non nuls de $r$ facteurs $b_{j}$ sont en nombre fini, et sont éléments de $A_{\gamma}$ (avec $\gamma=\beta^{r}$ ). Si de plus (avec les notations du théorème 5), $\prod_{i}$ ker $\varphi_{i}$ est un A-monel noethérien, 1 'anel $A_{\xi}[X]$ est noethérien.

$\S 3$ - MONOIDES NOETHERIENS.

a) Notion de monotde noethérien. Charpente d'un monotde :

Soit $D$ un monolde (ou demi-groupe) commutatif. On appellera D-idéal (ou idéal) de $D$ toute partie non vide $H$ de $D$, stable par multiplication par les éléments de $D$, c'est-à-dire, telle que $D H \subset H$. Une partie $S$ de $D$ est un système de D-générateurs de $\mathrm{H}$ si $\mathrm{H}$ est le plus petit idéal contenant $\mathrm{S}$, C'est-ä-dire, si $H=D S U S$ (resp. DS si $D$ possède un élément unité). Lorsque $S=\{a\}$, on notera (a) le D-idéal.

On dit que le monorde $D$ est noethérien si et seulement si tout D-idéal de $D$ est de type fini, c'est-à-dire possède un système fini de D-générateurs. Ceci équivaut évidemment à la condition de chaine croissante pour les D-idéaux. Par exenple, si la graduation propre d'un anel noethérien est un monorde, celui-ci est noethérien. Il est clair que la propriété noethérienne d'un monofide se conserve par un morphisne et dans un produit direct. Bien entendu, pour un D-idéal (qui est aussi un sous-monolde), on . distinguera système de générateurs (comme monoride) et système de D-générateurs (comme idéal)。

Deux éléments $a$ et $b$ d'un monorde $D$ seront dits associés au sens 
large si et seulement si les D-idéaux (a) et (b) sont égaux. Cela signifie que chacun des éléments a et b divise l'autre, ou lui est égal. L'association large est, évidemment, une relation d'équivalence sur $D$ compatible avec le produit dans $D$. Le monoide quotient sera appelé la charpente de D. On appellera charpente (ou hololde) tout monotde ou deux éléments associés au sens large sont egaux. Il est évident qu'un monolde est noethérien si et seulement si sa charpente $1^{\prime}$ est. Pour un D-idéal $\mathrm{H}$, appelons système minimal $\mathrm{T}$ de D-générateurs de $\mathrm{H}$ tout système ( $s^{\prime} i l$ en existe) de D-générateurs tels qu'aucum d'eux n'en divise un autre.

Lemme 1. Soit $\mathrm{H}$ un idéal d'une charpente D. Si H possede un systeme minimal $\mathrm{T}$ de $\mathrm{D}$-générateurs, tout autre système de $\mathrm{D}$-générateurs de $\mathrm{H}$ contient $\mathrm{T}$. En particulier, si $\mathrm{H}$ est de type fini, il possede un plus petit systeme de D-génerateurs.

Soit $S$ un autre système de D-générateurs de $H$. Quel que soit $x \in T$, il existe $s_{x} \in S$ tel que $\left(s_{x}\right) \supset(x)$, et il existe $y \in T$ tel que $(y)=\left(s_{x}\right)$. D étant une charpente, et $\mathrm{T}$ étant minimal, on a $\mathrm{y}=\mathrm{x}=\mathrm{s}_{\mathrm{x}}$, d'où $T \subset S$. Enfin, si $H$ admet un système fini de D-générateurs, on en extrait aisément un système minimal.

La relation d'association large est distincte de l'association au sens strict habituel (où a est produit de b par un élément inversible). on le voit en considérant le monorde des monomes en $x, y, z$ dans l'exemple suivant.

Soient $k$ un corps, et $A$ l'anneau noethérien des polynomes sur $k$, à trois variables $x, y, z$ liées par la relation $x-x y z=0$. Les idéaux $(x)$ et $(x z)$ de $I^{\prime}$ anneau $A$ sont égaux. Mais nous verrons que $x$ et $x z$ sont diviseurs propres l'un de l'autre, et que $x$ n'est pas un produit d'irréductibles. Supposons que $x$ et $x z$ soient associés stricts dans $A$, c'est-à-dire qu'il existe $f(x, y, z)$, élément inversible de $A$, tel que $x=x z$ f. Soit $g(x, y, z)$ un inverse de $f_{0}$. On a donc $x z=x g$, d'où l'on déduit $g=z+(1-y z) q_{0}(x, y, z)$, soit encore $g=z+(1-y z) q(y, z)$ car, multiplies par (1-yz) les termes en $x$ de $q_{0}$ disparaissent. De $x=x z f=x z y$, on déduit $z f=z y+(1-y z) \quad D_{0}(x, y, z)$. Donc $z$ est en facteur dans $p_{0}$, et $l^{\prime}$ on $a$ $f=y+(1-y z) \quad p_{1}(x, y, z)$, donc de même que plus haut : $f=y+\left(\begin{array}{ll}1-y & z\end{array}\right) \quad p(y, z)$. Donc $f$ et $g$ appartiendraient au sous-anneau $\mathbf{k}[y, z]$, dont les seuls inversibles sont les scalaires. On a une contradiction. Donc $x$ et $x z$ ne sont pas associés stricts. D'autre part, tout élément $p(x, y, z)$ de A s'écrit $p=q(y, z)+x r(x, y, z)$, avec unicité pour le polynôme $q$, puisque $x$ est en facteur dans $x=x y z$. Si $x$ était un produit d'irréductibles, 
soit $x=\prod_{i=1}^{n} p_{i}$ avec $n \geqslant 1$, et, pour tout $i, p_{i}$ irréductible et égal à $q_{i}(y, z)+x r_{i}(x, y, z)$. On en déduit $x=\prod_{i=1}^{n} q_{i}(y, z)+x R(x, y, z)$.

L'unicité précédente montre que $\prod_{i=1}^{n} q_{i}=0, d^{\prime}$ ou, puisque $k[y, z]$ est intègre, l'existence de $i_{0}$ tel que $q_{i_{0}}=0$. Donc, l'irréductible $p_{i_{0}}$ serait divisible par x. Mais $x n^{\prime}$ est pas irréductible, car on sait que $x$ et $x z$ sont diviseurs propres l'un de l'autre. D'autre part, $x n^{\prime}$ est pas inversible, comme diviseur de zéro. Alors, que $x$ soit associé à $P_{i}$ ou en soit un diviseur propre, $p_{i}$ ne peut pas être irréductible, ce qui est une contradiction. Dans l'anneau noethérien $A, x n^{\prime}$ est pas produit d'irréductibles.

b) Le graphe d'une charpente noethérienne : Pour un élément $x$ d'une charpente $D$, les puissances $\left\{x^{n}\right\}_{n} \geqslant 1$ sont, ou toutes égales à partir d'un exposant e, appelé indice stationnaire de $x$, ou toutes différentes (on pose alors $e=\infty$ ). En effet, si par exemple $x^{i}=x^{j}$ avec $1 \leq i<j$, on en déduit (D étant une charpente), $x^{i}=x^{i+1}$, et enfin $x^{i}=x^{n}$ pour tout $n \geq i$.

Lemme 2 (des unités) : Soient D une charpente noethérienne, et $x$ et $a_{0}$ deux éléments de $D$. Les propriétés suivantes sont équivalentes : (a) $x$ est unité de $a_{0} ;(b)$ il existe une suite $\left\{a_{n}\right\}_{n} \geq_{0}$ d'éléments de $D$ tels que, quel que soit $n, a_{n}=x a_{n+1}$. Au contraire, si $x n^{\prime}$ est pas unité de $a_{0}$, il existe $j \geq 0$ et $b \in D$ tels que $a_{0}=x^{j} b$ et que $x$ ne divise pas $b$.

Si (a) est vérifiée, on pose $a_{n}=a_{0}$, pour tout $n$. Inversement, comne $D$ est noethérienne, la suite croissante d'idéaux $\left(a_{n}\right)$ est stationnaire à partir d'un entier s。 Quel que soit $n \geq s, a_{n}=a_{s}$. On a alors $a_{0}=x^{s+1} a_{s+1}=x x^{s} a_{s}=x a_{0}$. La deuxième assertion est évidente. Si $x$ et $a_{0}$ vérifient les propriétés équivalentes du lemme, on dira que $x$ divise $a_{0}$ indéfiniment. Cela implique la propriété : (c) toute puissance de $x$ divise $a_{0}$. Si l'indice stationnaire de $x$ est fini, (a) et (c) sont équivalentes. Il $n^{\prime} e n$ est pas forcément ainsi lorsqu'il est infini.

Dans l'étude qui va suivre, on suppose dans la charpente noethérienne D l'existence d'un élément unité, ce qui ne modifie en rien les résultats et leur généralité, mais allège parfois la rédaction. Dans le cas contraire, l'essentiel est de se souvenir que l'expression a divise b signifie $(a) \supset(b)$ (donc soit $b=c a, c \in D$; soit $a=b$ ). 
On va définir, pour tout élément $\alpha$ de l'ensemble $\Omega$ des ordinaux inférieurs strictement à un certain ordinal $\lambda$, un ensemble $R_{\alpha}$ de facteurs résiduels ou résidus d'indice $\alpha_{\text {. }}$ On notera $D_{\alpha}$ le $D$-idéal engendré par $R_{\alpha}$, et, D étant nocthérien, $\mathrm{x}_{\alpha} \subset \mathrm{R}_{\alpha}$ le plus petit système, fini, de $D$-générateurs de $D_{\alpha}$. On notera $M_{\alpha}$ le sous-monoìde de $D$ engendré par $U_{\alpha}=\underset{\beta<\alpha}{U} X_{\beta}$, c'est-à-dire l'ensemble des produits finis de puissances non toutes nulles d'éléments de $\mathrm{U}_{\alpha} \cdot \mathrm{X}=\mathrm{U}_{\alpha<\lambda} \mathrm{X}_{\alpha}$ sera un système de générateurs du monoìde $\mathrm{D}$. On pose d'abord $R_{1}=D_{1}=D$, donc $x_{1}=\{1\}$, puis $R_{2}=D_{2}=D_{0} .\{1\}$.

\section{Définition des suites de factorisation et des résidus. Pour un}

ordinal $\sigma \in \Omega$, supposons connus :

(10) Pour tout $d \in D$, au moins une $\sigma$-suite de factorisation de d, c'est-à-dire que pour chaque $\alpha<\sigma($ et $\alpha \geq 2)$ est connu un couple $\mathrm{C}_{\alpha}=\left\{m_{\alpha}, \mathrm{E}_{\alpha}\right\}$ dans lequel $m_{\alpha} \in D$, et $E_{\alpha}$ ou est vide, ou contient un seul élément $d_{\alpha} \in D ; d_{\alpha}$ est appelé un résidu d'indice $\alpha$ de d. On prend $C_{\alpha}(1)=(1, \emptyset)$ et, si $d \neq 1, c_{2}(d)=(1, d)$.

(20) Pour tout $\alpha<\sigma$, l'ensemble $R_{\alpha}$ des résidus, et aussi l'idéal $D_{\alpha}$, le système $\mathrm{x}_{\alpha}$, et le monorde $\mathrm{M}_{\alpha}$.

Nous supposons de plus que ces éléments connus vérifient les conditions suivantes :

(a) Quel que soit $\alpha<\sigma, R_{\alpha}$ est l'ensemble des résidus $d_{\alpha}$, pour tous les $d \in D$, et pour toutes les $\sigma$-suites de factorisation de $d$.

Quel que soit $a \in D$, quelle que soit la $\sigma$-suite de factorisation de $d$, et quel que soit 1 ordinal $\alpha<\sigma$ :

(b) $m_{\alpha} \in M_{\alpha}$; si $E_{\alpha}=\emptyset, d=m_{\alpha}$; sinon, $d_{\alpha} \notin M_{\alpha}$ et on a $d=m_{\alpha} d_{\alpha}$ (donc on a $D=M_{\alpha} \cup D_{\alpha}$ ).

(c) Si $\alpha$ est de première espece et si $E_{\alpha-1} n^{\prime}$ est pas vide : ou bien $d_{\alpha-1} \in M_{\alpha}$, et on a $c_{\alpha}=\left\{m_{\alpha-1} d_{\alpha-1}, \emptyset\right\}$.

(d) ou bien $d_{\alpha-1} \notin M_{\alpha}$, et on a $d_{\alpha-1}=p_{\alpha-1} d_{\alpha}, m_{\alpha}=m_{\alpha-1} p_{\alpha-1}$; $p_{\alpha-1}$ egale 1 ou appartient au monoride engendré par $x_{\alpha-1} ; d_{\alpha}$ est étranger a certains éléments de $x_{\alpha-1}$ (notamment ceux qui figurent dans $p_{\alpha-1}$ ) et admet les autres (un au moins) pour unités.

(e) Si $\alpha$ est de seconde espece, il existe $\beta<\alpha$ tel que $C_{\beta}=C_{\alpha}$.

(f) S'il existe $\beta<\alpha$ tel que $E_{\beta}=\varnothing$, on a $C_{\beta}=C_{\alpha^{*}}$

On dit que la $\sigma$-suite est achevée s'il existe $\varphi<\sigma$ tel que $E_{\varphi}=\emptyset$, et qu'elle s'achève en $\varphi$, si $\varphi$ est le plus petit ordinal vérifiant cette condition. Noter que $\varphi$ est de première espèce. 
Alors, montrons pour tout $d \in D$ qu'une $\sigma$-suite de factorisation de $d$ se prolonge en une $(\sigma+1)$-suite, c'est-à-dire qu'il existe un couple $C_{\sigma}$ convenable, et montrons l'existence d'un ensemble $R_{\sigma}$ de résidus d'indice $\sigma$. $S^{\prime} i l$ existe $B<\sigma$ tel que $E_{\beta}$ soit vide, on pose $C_{\sigma}=C_{B^{*}}$. Etudions le cas contraire.

Lorsque $\sigma$ est de première espèce, notons $x_{\sigma-1}=\left\{x_{\sigma-1, i}\right\}_{i \in I}$ ou plus simplement $\left\{x_{i}\right\}_{i \in I}$, le plus petit système (fini) de D-générateurs de $D_{\sigma-1}$. Soit $I^{0}=\left\{i \in I ; x_{i}\right.$ divise $\left.d_{\sigma-1}\right\}$. Comme $d_{\sigma-1} \in D_{\sigma-1}$, I० n'est pas vide. Procédons par disjonction des cas :

(1) $\operatorname{si~} d_{\sigma-1} \in M_{\sigma}$, on prend $C_{\sigma}=\left\{m_{\sigma-1} d_{\sigma-1}, \emptyset\right\}$.

(2) Si $d_{\sigma-1} \notin M_{\sigma}$ et si, quel que soit $i \in I^{0}, x_{i}$ est unité de $d_{\sigma-1}$, on pose $\mathrm{C}_{\sigma}=\mathrm{C}_{\sigma-1}$

(3) Si $d_{\sigma-1} \notin M_{\sigma}$ et $s^{\prime} i l$ existe $i_{1} \in I^{\circ}$ tel que $x_{i}$ ne soit pas unité de $\mathrm{d}_{\sigma-1}$ : le lemme des unités montre 1 'existence dé $j_{1} \geq 1$ et de $b_{1} \in D$ tels que $d_{\sigma-1}=x_{i_{1}}^{j} b_{1}$ et que $x_{i}$ ne divise pas $b_{1}$. Alors, $a_{\sigma-1} \not M_{\sigma}$ implique $b_{1} \notin M_{\sigma}$ (qui contient $M_{\sigma-1}$ ), donc $b_{1} \in D_{\sigma-1}$. Donc $I^{1}=\left\{i \in I ; x_{i}\right.$ divise $\left.b_{1}\right\}=\left\{i \in I^{\circ} ; x_{i}\right.$ divise $\left.b_{1}\right\}$ est non vide. On poursuit I'étude avec $b_{1}$ et $I^{1}$ comme avec $d_{\sigma-1}$ et $I^{\circ}$. Finalement on obtient une partie non vide $\left(\operatorname{car} \mathrm{d}_{\sigma-1} \notin \mathrm{M}_{\sigma}\right)^{-1} I^{\mathrm{n}}$ de $I$, un élément $\mathrm{b}_{n} \notin \mathrm{M}_{\sigma}$ et une factorisation $\mathrm{d}_{\sigma-1}=\mathrm{P}_{\sigma-1} \mathrm{~b}_{n}$ avec

$\mathrm{P}_{\sigma-1}=\prod_{k=1}^{n} x_{i_{k}}^{j_{k}}$ où les $i_{k} \notin I^{n}$, où les $x_{i}$ pour $i \notin I^{n}$ ne divisent pas $b_{n}$, et où les $x_{i}$ pour $i \in I^{n}$ sont unités pour $b_{n}$. on pose alors $d_{\sigma}=b_{n}$, et $m_{\sigma}=m_{\sigma-1} P_{\sigma-1}$. On a donc $d_{\sigma} \notin \mathbb{M}_{\sigma}$ et $m_{\sigma} \in M_{\sigma}$. Noter que $d_{\sigma}$ peut dépendre du choix des indices $i, \ldots .$.

Lorsque $\sigma$ est de seconde espèce, la chaine d'idéaux $\left\{\left(d_{\alpha}\right)\right\}_{\alpha<\sigma}$ est croissante, donc stationnaire car $D$ est noethérien. Donc il existe un ordinal $\beta<\sigma$ tel que $\beta \leq \alpha<\sigma$ implique $d_{\beta}=a_{\alpha}$ on pose alors $C_{\sigma}=C_{\beta^{*}}$. Puisque $M_{\sigma}=\bigcup_{\alpha<\sigma} M_{\alpha}$, on a $m_{\sigma} \in M_{\sigma}$, et $d_{\beta} \notin M_{\alpha}$ pour $\beta \leqslant \alpha<\sigma$ implique $d_{B} \notin M_{\sigma}$. Il est clair que la $(\sigma+1)$-suite déterminée pour d vérifie les conditions annoncées.

$$
\text { Par définition, } R_{\sigma} \text { est I'ensemble des résidus } d_{\sigma} \text { ainsi définis }
$$
pour tous les $d \in D$, et pour toutes les $(\sigma+1)$-suites de factorisation de $d$. Il est clair que la famille des monoides $M_{\alpha}$, ordonnée par inclusion, est 
strictement croissante. Donc, il existe un ordinal $\lambda$ tel que $M_{\lambda}=D_{\text {. Donc, }}$ toute $\lambda$-suite d'un $d \in D$ est achevée et donne une factorisation $m_{\lambda}$ de $d$ (pas forcément unique), par les éléments de $X=\underset{\alpha<\lambda}{U} x_{\alpha^{\circ}}$ Le fait qu'il puisse exister des $\lambda$-suites, ou des factorisations autres que celles envisagées dans l'étude précédente, n'interviendra pas dans les démonstrations ultérieures. Appelons $\mathrm{x}_{\alpha}$ la couche d'indice $\alpha$.

Propriété d'absorption dans les couches $\mathrm{x}_{\alpha}$. Soit $\mathrm{d} \in \mathrm{x}_{\sigma}$, et considérons une $(\sigma+1)$-suite de factorisation de d. Certainement $E_{\sigma}$ est non vide, sinon $d \in M_{\sigma}$ et ne serait pas un résidu d'indice $\sigma$. Donc $d=m_{\sigma} d_{\sigma}$,

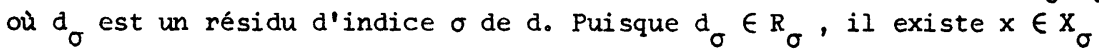
tel que $x$ divise $d_{\sigma}$ qui divise $d . x_{\sigma}$ étant le plus petit système de $D$-générateurs de $D_{\sigma}$, on a $x=d_{\sigma}=d$, et $d=m_{\sigma} d$. Si $m_{\sigma}$ est différent de 1 , soit $\mathrm{X}_{\alpha}$ la première couche dont des éléments figurent dans $\mathrm{m}_{\sigma}$, et soit $\mathrm{z}$ le premier élément de cette couche mis en facteur dans la $(\sigma+1)$-suite par le procédé (3) de factorisation. Mais $d=m_{\sigma} d$ implique $d=z d$; donc (lemme 2) $z$ est unite pour $d$, ce qui contredit (3). Donc on a $m_{\sigma}=1$.

Remarque : Les éléments d'une couche $\mathrm{X}_{\sigma}$ quelconque sont des résidus d'indice $\alpha$, pour tout $\alpha \leqslant \sigma$.

Lemme 3 (d'absorption): Quelles que soient les couches $\mathrm{x}_{\alpha}$ et $\mathrm{x}_{\beta}$, $\alpha<\beta$, tout élément $x_{\beta}$ de $x_{\beta}$ absorbe certains éléments, et un au moins, de $x_{\alpha}$,et $n$ 'est divisé par aucun des autres.

En particulier, entre éléments de $\mathrm{X}, \mathrm{x}$ divise $\mathrm{y}$ signifie que $\mathrm{y}$ absorbe $x$, que $x$ est unité de $y$.

On constate maintenant que les couches $\mathrm{x}_{\alpha}$ et les idéaux $D_{\alpha}$ peuvent être déterminés de façon plus simple. On pose $Y_{1}=$ D. Puis, si $\alpha \geqslant 2$ est de première espèce, soit $Y_{\alpha} I^{\prime}$ 'ensemble des éléments de $Y_{\alpha-1}$ qui admettent certains éléments (un au moins) de $X_{\alpha-1}$ comme unités, et qui ne sont pas multiples des autres. Si $\alpha$ est de seconde espèce, on pose $Y_{\alpha}=\bigcap_{\beta<\alpha} Y_{\beta}$.
Il est clair que $Y_{\alpha} \subset R_{\alpha}$ et le lemme 3 montre que $X_{\alpha} \subset Y_{\alpha}$.Donc $\quad Y_{\alpha}$ engendre le D-idéal $D_{\alpha}$ et détermine la couche $X_{\alpha}$. La definition des couches est donc indépendante de celle des suites de factorisation. Il en résulte que l'ensemble $X$ de générateurs de la charpente $D$, la partition de $\mathrm{X}$ en un ensemble bien ordonné de couches finies $\mathrm{X}_{\alpha}$, et les conditions d'absorption (lemne 3 ) sont uniquement déterminés par $D$. La donnée de $\mathrm{X}$, de sa partition $\left\{\mathrm{x}_{\alpha}\right\}_{\alpha \in \Omega}$ et des conditions d'absorption sera appelée le graphe $X$ de la charpente noethérienne D. Et si M est le monorde commutatif, 
libre au sens de Ljapin ([5], chap. IX), engendré par X et les relations d'absorption, $D$ est un monolde quotient de $M$.

On appelle partie transverse d'un monorde $D$ toute partie non vide $T$ de $D$ dont aucun élément $n$ 'est multiple d'un autre (c'est-à-dire, $\forall a$ et $b \in T$, $a \neq b$, les idéaux $(a)$ et (b) ne sont pas comparables). Par exemple, une couche $\mathrm{x}_{\alpha}$ est une partie transverse. Une partie d'une partie transverse est transverse.

Lemme 4 : Le graphe d'une charpente noethérienne ne contient pas de partie transverse infinie.

$$
\text { Si } T=\left\{x_{n}\right\}_{n \in \mathbb{N}} \text { est une partie transverse infinie d'un monorde } M \text {, }
$$

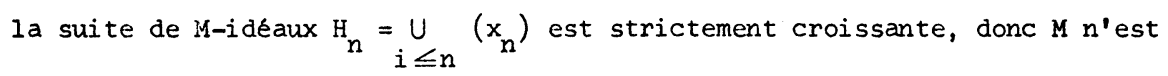
pas noethérien. En particulier, si $D$ est une charpente noethérienne, toute partie transverse de $D$, donc de son graphe $X$, est finie.

\section{c) Le théorème fondamental :}

Theorème : Une charpente (commutative) D est noethérienne si et seulement si elle est inage homomorphe d'une charpente $M$ vérifiant les conditions suivantes : Il existe un système $\mathrm{X}$ de générateurs du monorde commutatif $\mathrm{M}$, et une partition de $\mathrm{X}$ en un ensemble bien ordonné de couches finies non vides $\mathrm{x}_{\alpha}$, ensemble indexé par les ordinaux strictement inférieurs

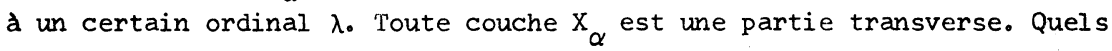
que soient les oráinaux $\alpha$ et $\beta$ tels que $\alpha<\beta<\lambda$, quel que soit $y \in x_{\beta}$, il existe une partie non vide $\mathrm{x}_{\alpha}(\mathrm{y}) \subset \mathrm{x}_{\alpha}$ telle que $\mathrm{x} \in \mathrm{x}_{\alpha}$ (y) équivaut à $x y=y$. Ces relations, et celles de commutativité, sont les seules existant entre les éléments de $\mathrm{X}$. Enfin, toute partie transverse de $\mathrm{X}$ est finie.

Un ensemble $X$ vérifiant les conditions du théorème, sauf peut-être la dernière (finitude des parties transverses), sera appelé un graphe de générateurs. On dira que le graphe est noethérien g'il n'a pas de partie transverse infinie. Le thérème s'énonce alors plus brièvement : Une charpente D est noethérienne si et seulement si elle est une image homomorphe du monoide "libre" engendré par un graphe noethérien.

Nous avons vu que la condition cst nécessaire. Il reste a montrer qu'elle est suffisante. soit donc $X$ un graphe de générateurs, et $M$ le monorde "libre" qu'il engendre. Supposons que M possède une partie transverse infinie $P$, et montrons qu'il en est de même pour $X$. D'abord, voyons deux lemmes préliminaires.

Si $m \in M$, il est un produit de puissances d'un nombre fini de générateurs appartenant à $X$, qui forment une partie transverse. En effet, si 
I'un $x$ de ces générateurs en divise un autre $y, x$ est unité pour $y$, et peut être omis dans le produit. Nous dirons qu'une telle décomposition de $m$ est réduite (ou irréductible). Une décomposition réduite est unique. Sinon, deux décompositions réduites distinctes de $m$ donneraient une relation supplémentaire entre éléments de $\mathrm{X}$, autre que celles imposées (voir théorème) à un graphe.

Lemme 5 : (1) Si un générateur $x \in X$ divise un élément $m$ de $M$, il divise (c'est-à-dire est unité pour, ou est égal à) un facteur de la décomposition réduite de $m$.

(2) Soient $m \in M,\left\{x_{1}, \ldots, x_{s}\right\}$ une partie transverse de $x$, et $n_{1}, \ldots, n_{s}$ des entiers $\geq 1$. Si, pour tout $i=1, \ldots, s, x_{i}{ }_{i}$ divise $m$, alors $\prod_{i=1}^{s} x_{i}{ }^{i}$ divise $m$.

Si $m=x^{e} p$, avec $e \geq 1$, de la décomposition réduite de $p=\prod_{j=1}^{r} z_{j}^{e_{j}}$, déduisons comme ci-dessus celle de $m$; si donc $x n^{\prime}$ 'est pas unité d'un $z_{j}, x$ figure dans la décomposition réduite de $m$, avec un exposant $\geq e$ 。 Si $x_{i}$ est unité pour un facteur de la décomposition réduite de $m$, il l'est pour $m$, et $l^{\prime}$ 'on a $m=x_{i}^{n_{m}}$. Dans le cas contraire, $x_{i}$ figure dans la décomposition réduite de $m$, avec un exposant $\geq n_{i}$, d'où le résultat. Remarquons que si $x \in X$ est tel que $x^{n+1}$ ne divise pas $m, x$ 'est pas unité de $m$; si donc $m=x^{n} p, p$ est unique.

Lemme 5 : Soit $P$ une partie transverse infinie de $M$.

(1) Quel que soit $P$ dans $P$, sa décomposition réduite contient au moins un générateur $y \in X$, avec l'exposant $e \geq 1$, tel qu'il existe une partie infinie $\mathrm{U}\left(\mathrm{y}^{e}\right)$ de $\mathrm{P}$ dont $\mathrm{y}^{\mathrm{e}}$ ne divise aucun élément. Lorsqu'un générateur $\mathrm{y} \in \mathrm{X}$ vérifie cette condition pour au moins un élément $\mathrm{P}$ de $\mathrm{P}$, il est dit facteur régulier de $\mathrm{P}$.

(2) Si y est un facteur régulier de $P$, il existe un entier $k, 0 \leqslant k \leqslant e-1$, et une partie $V$ de $M$ dont $y$ ne divise aucun élément, tels que $y^{k} V$ soit une partie infinie de $P$ (donc $V$ est infinie transverse) et que tout facteur $z$ régulier pour $V$ soit régulier pour $P$.

(3) $P$ possède une infinité de facteurs réguliers.

$$
\text { Soit } \mathrm{p}=\prod_{j=1}^{s} \mathrm{y}_{j} \mathrm{e}_{j} \text { la décomposition réduite de } \mathrm{p} \text {, avec les } \mathrm{y}_{j} \in \mathrm{X} \text {. }
$$

Pour chaque $j$, notons $F_{j}$ l'ensemble des éléments de $P$ que $y_{j}{ }_{j}$ ne divise pas. 
Si tous les $F_{j}$ étaient finis, leur réunion $F$ le serait aussi, et $P_{\circ} . F$ serait non vide. Donc, pour un $h \in P_{0} . F$, tout $y_{j}{ }_{j}$ diviserait $h$, et $p$ diviserait $h$ (lemme 5) ; P ne serait pas transverse.

Si y est régulier pour $P$, d'exposant e, pour tout entier $i$, $0 \leqslant i \leqslant$ e -1 , notons $U_{i}$ la partie de $U\left(y^{e}\right)$ formée des éléments divisibles par $y^{i}$, mais non par $y^{i+1}$. Comme $U\left(y^{e}\right)$ est la réunion des $U_{i}$, $I^{\prime} u n$ de ces ensembles, soit $U_{k}$, est infini. Lorsque $k=0, v=U_{0}$ convient évidemment. Lorsque $k \geq 1$, pour chaque $u \in U_{k}, y^{k+1}$ ne divise pas $u$, donc $y n^{\prime}$ est pas

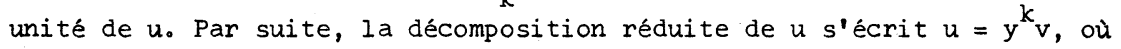
$v \in M$ est unique et écrit sous forme réduite, où y ne divise pas $v$, où tout générateur $g$ figurant dans $v$ ne divise pas $y$ (en particulier $\{y, g\}$ est transverse). On prend pour V l'ensemble de ces éléments $v$ ainsi définis, pour $u$ parcourant $U_{k}$. On a $U_{k}=y^{k} v$, et $V$ est infini transverse. Toujours pour $\mathrm{k} \geq 1$, soit $\mathrm{z}$ un facteur régulier pour $\mathrm{V}$, avec l'exposant $\mathrm{a} \geq 1$ dans $v_{0} \in V_{0}\{y, z\}$ étant transverse, $z^{n}$ divise un $v \in V$ si et seulement si $y^{k} z^{n}$ divise $y^{k} v$. Donc, $z$ est facteur de $u_{0}=y^{k} v_{0}$ avec $1^{\prime}$ exposant a i et si $\mathrm{z}^{\mathrm{a}}$ ne divise pas un $\mathrm{v} \in \mathrm{V}$, il ne divise pas $\mathrm{y}^{\mathrm{k}} \mathrm{v} \in \mathrm{U}_{\mathrm{k}}$. Donc $\mathrm{z}$ est facteur régulier pour $P$.

Notons $\mathrm{y}_{1}$ un facteur régulier de $\mathrm{P}$, et $\mathrm{V}_{1}$ la partie infinie transverse associée à $y_{1}$ par l'assertion (2) du lemme. Supposons connus $n$ facteurs réguliers distincts $y_{1}, \ldots . . . y_{n}$ de $P$, et un ensemble infini transverse $v_{n}$ dont $y_{i}$ ne divise aucun élément, quel que soit $i=1, \ldots, n$, et dont tout facteur régulier soit régulier pour $P$. Prenons alors un facteur $y_{n+1}$ régulier pour $v_{n}$, et soit $v_{n+1} l$ 'ensemble infini transverse qui lui est associé par (2) : On sait qu'il existe un entier $k$ tel que $y_{n+1}^{k} v_{n+1}$ soit une partie infinie de $v_{n}$, que pour tout $i \leqslant n, y_{i}$ est distinct de $y_{n+1}$ ' que $y_{n+1}$ ne divise aucun élément de $v_{n+1}$, et que tout facteur régulier

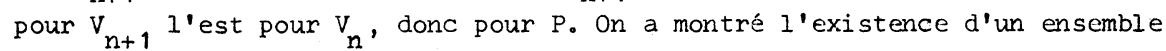
infini $Y=\bigcup_{n \geqslant 1}\left\{y_{n}\right\}$ de facteurs réguliers de $P$ 。

Achevons la démonstration du théorème. Dans la construction précédente de $Y$, posons $Y_{n}=\left\{y_{1}, \ldots, y_{n}\right\}, V_{0}=P$, et pour tout $n \geq 0$, notons $F_{n}$ l'ensemble des facteurs réguliers pour $V_{n}$. Nous savons que, pour tout $n \geq 0, F_{n}$ est infini et $F_{n}$ contient $F_{n+1}$. Les couches $x_{\alpha}$ sont bien ordonnées, et, pour tout $n \geq 0$, il existe un plus petit ordinal $\alpha_{n+1}$ tel que $F_{n} \cap x_{\alpha_{n+1}}$ soit non vide; prenons $y_{n+1}$ dans $F_{n} \cap x_{\alpha_{n+1}}$. Alors, quel que soit $x \in F_{n+1} \subset F_{n}$, on a $\alpha_{n+1} \leqslant \alpha(x)$, si $\alpha$ (x) désigne l'ordinal de la couche de $x$. En particulier, si $Y_{n}$ est transverse, pour tout $i \leqslant n$ on a 
$\alpha_{i} \leq \alpha_{n+1}$, donc $y_{n+1}$ ne divise pas $y_{i}$ (c'est-à-dire $n^{\prime}$ en est pas unité). $D^{\prime}$ autre part, pour tout $i \leq n, y_{i}$ ne divise aucun élément de $v_{i}$ (lemme $\sigma$ ), donc de $F_{i}$, donc ne divise pas $y_{n+1}$. Cela entraine que $Y_{n+1}$ aussi est transverse. Donc, pour tout $n \geq 1, Y_{n}$ est transverse. La réunion $Y=\underset{n \geq 1}{U} Y_{n}$ est donc une partie infinie transverse du graphe.

On connait donc la structure de toute charpente noethérienne. Pour passer d'une charpente $C$ à un monolde noethérien $D$ admettant $C$ pour charpente, il faut opérer une sorte de "dilatation" de la charpente. Pour tout $t \in C$, on se donne un ensemble $D_{t}$, ces ensembles étant deux à deux disjoints ; on note $D$ leur réunion. Puis, pour tout couple $\left(t, t^{\prime}\right) \in C^{2}$ on se donne une application $\varphi_{t, t^{\prime}}: D_{t} \times D_{t} \rightarrow_{t t^{\prime}}$ vérifiant d'abord les conditions suivantes (commutativité et associativité) : Quels que soient $t$, $t$ ' et $t$ " dans $C$, quels que soient a dans $D_{t}$, a' dans $D_{t}$, et a" dans $D_{t " \text { " }}$ on $a: \varphi_{t, t^{\prime}} \cdot\left(a, a^{\prime}\right)=\varphi_{t^{\prime}, t^{\prime}} \cdot\left(a^{\prime}, a\right)$ $\varphi_{t t^{\prime}, t^{\prime \prime}} \cdot\left[\varphi_{t, t^{\prime}} \cdot\left(a, a^{\prime}\right), a^{\prime \prime}\right]=\varphi_{t, t^{\prime} t^{\prime \prime}} \cdot\left[a, \varphi_{t} t^{\prime \prime} \cdot\left(a^{\prime}, a^{\prime \prime}\right)\right]$ Ensuite, pour tout $t \in C$, notons $E_{t}=\left\{t^{\prime} \in C ; t t^{\prime}=t\right\}$ et $U_{t}=U_{t, \in E_{t}} D_{t}$. On a $E_{t}^{2} \subset E_{t} d^{\prime}$ ou $U_{t}^{2} \subset U_{t}$ et, (par définition de $E_{t}$ ), quel que soit $d \in E_{t}$, $d U_{t} \subset D_{t}$. Soient d et $d^{\prime}$ dans $D_{t}$ vérifiant $d^{\prime}=d u ; s i$ $u \in D_{\theta}$, on doit avoir $t \theta=t$, donc $u \in U_{t}$. Donc, pour que les éléments de tout $D_{t}$ soient associés entre eux au sens large, il faut et il suffit que : (AI) quels que soient $t \in C$ et $d \in D_{t}$, on ait $d U_{t}=D_{t}{ }^{\circ}$

Cette dernière condition peut s'exprimer autrement. Supposons maintenant que pour tout $t \in C$ un élément au moins $x=x(t) \in D_{t}$ vérifie $x U_{t}=D_{t}$. La relation $x u=x u^{\prime}$ définit sur $U_{t}$ une relation d'équivalence pour laquelle $\bar{u}$ désignera la classe de $u$, et $\bar{U}_{t}$ l'ensemble quotient. La condition (Al) s'écrit alors : pour tout $t \in C$, pour tout $u \in U_{t}$, on a $x u U_{t}=x U_{t}$, ce qui équivaut à $\bar{u} \bar{U}_{t}=\bar{U}_{t}$, donc au fait que $\bar{U}_{t}$ soit un groupe. Finalement, la condition (Al) d'association large peut être formulé ainsi : quel que soit $t \in C$, il existe $x \in D_{t}$ vérifiant les conditions $x U_{t}=D_{t}$, et $\bar{U}_{t}$ est un groupe. Bien entendu, ces conditions, que $l^{\prime}$ 'on peut expliciter, sont imposées aux fonctions $\varphi_{t, t}{ }^{\circ}$

d) Exemples d'annéldes noethériens pas forts :

Dans les exemples qui suivent, nous allons montrer qu'un annélde noethérien peut être ni fort, ni strict, et qu'il peut etre strict, mais pas fort (Chap. II, $\S 1$ ). I,'annérde construit A aura ses domaines d'addibilité 
isomorphes au groupe additif du corps à deux éléments, et sera déterminé par la donnée de sa graduation propre D (commutative) (voir chap. I, $\S 2$, exemple $3^{\circ}$ ). On pourra prendre $D$ pour support de $A$. On se donne le graphe noethérien suivant : $\mathrm{X}=\bigcup_{\alpha<\lambda} \mathrm{x}_{\alpha}$, avec $\mathrm{x}_{1}=\left\{1=\mathrm{e}_{1}\right\}, \mathrm{x}_{2}=\left\{\mathrm{e}_{2}, \mathrm{x}\right\}$ et pour $2<\alpha<\lambda, x_{\alpha}=\left\{e_{\alpha}\right\}$, en supposant que $x$ n'est unité pour aucun élément du graphe (donc, pour $\alpha<\beta<\lambda$, on a $e_{\alpha} e_{\beta}=e_{\beta}$, et aussi $1 \mathrm{x}=\mathrm{x}$ ).

$\left.1^{\circ}\right)$ On suppose infini le cardinal de $\lambda$. On considère le monoide "libre" du graphe $X$, et soit $D$ son quotient par les relations suivantes : Tout élément $e_{\alpha}$ est idempotent. Une suite $\left\{\lambda_{n}\right\}_{n \geqslant 1}$ strictement croissante d'ordinaux $<\lambda$ est donnée, et quel que soit $i \geqslant 1$, quels que soient $\alpha$ et $\beta<\lambda_{i}$ ' on a $x^{i} e_{\alpha}=x^{i} e_{\beta}$. En fait, seules les puissances de $x$ agglutinent. On voit que, lorsque $I^{\prime}$ exposant $i$ augmente, $x^{i}$ agglutine a 1 un ensemble de génerateurs $e_{\alpha}$ de plus en plus important. Donc $\times n^{\prime}$ est pas quasi-régulier strict, ni quasi-régulier, et l'annérde noethérien A n'est ni strict, ni fort.

$\left.2^{\circ}\right)$ On suppose toujours infini le cardinal de $\lambda$. On suppose donnée une suite $\left\{\lambda_{i}\right\}_{i \geq 1}$ strictement croissante d'ordinaux $<\lambda$, tout intervalle $\left[\lambda_{i}, \lambda_{i+1}[\right.$ étant partagé en $i$ intervalles par une suite $\lambda_{i}{ }^{0}=\lambda_{i}<\lambda_{i}{ }^{1}<\ldots \ldots<\lambda_{i}^{i-1}<\lambda_{i}^{i}=\lambda_{i+1}$ on considere le monotde "libre" du graphe $X$, et soit $D$ son quotient par les relations suivantes : Tout élément $e_{\alpha}$ est idempotent. Quels que soient les entiers $n \geqslant 1$ et $i \geqslant 1$, quels que soient $\alpha$ et $\beta \in\left[\lambda_{i}, \lambda_{i}^{n}\left[\right.\right.$ (ou $\left[\lambda_{i}, \lambda_{i+1}[\right.$ si $n>i)$, on a $x^{n} e_{\alpha}=x^{n} e_{\beta}$.

$x^{n+1}$ agglutine strictement plus que $x^{n}$, donc $x n^{\prime}$ est pas quasirégulier, et A n'est pas fort. Cependant, A est strict. Seuls les $x^{n}$ (et

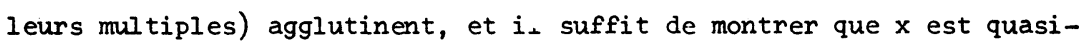
régulier strict. Montrons d'abord cela relativement à l'ensemble $X$ des générateurs. Soit $e_{\alpha} \in \mathrm{X}$ un générateur tel que $\alpha$ appartienne à un intervalle $\left[\lambda_{i}, \lambda_{i_{\alpha}+1}\left[\right.\right.$. Par définition, seuls pourront être agglutinés à e $e_{\alpha}$ les générateurs e $e_{\beta}$ pour lesquels $\beta \in\left[\lambda_{i}, \lambda_{i_{\alpha}+1}[\right.$, et ils seront tous agglutinés par $x^{i} \alpha$. En posant $i\left(x, e_{\alpha}\right)=i_{\alpha^{\prime}}$, on voit que $x$ est quasi-régulier strict relativement a $X$. Enfin, un élément a quelconque est un produit fini d'idempotents $e_{\alpha}$, et éventuellement d'une puissance de $x: a=x^{m} \prod_{\alpha \in I} e_{\alpha}$. Soit $J$ la partie de I correspondant aux $e_{\alpha}$ susceptibles d'agglutination (c'est-à-dire aux o appartenant à un intervalle $\left[\lambda_{i}, \lambda_{i_{\alpha}+1}[\right.$ ), et posons : 
$i(x, a)=\sup \left[0, \sup _{\alpha \in J}\left(i \alpha^{-m}\right)\right]$. Alors, $x$ est quasi-régulier strict pour $i(x, a)$.

$\left.3^{\circ}\right)$ Donnons-nous un monorde cominutatif quelconque $D$ dont tout élément est périodique, c'est-à-dire que pour tout $d \in D$, il existe des. entiers $n(d)$ et $p(d) \geq 1$ tels que $d^{n(d)}+p(d)=d^{n(d)}$. Alors, tout annéride $A$ de graduation $D$ est fort, bien qu'il puisse n'être pas noethérien. 


\section{Chapitre V}

\section{ANNEIDES NOETHERIENS FOR'TS}

On suppose toujours comnutatif's les annérdes considérés, ainsi que leurs graduations éventuelles.

\section{$\$ 1$ - DECOMPOSITION PRIMAIRE.}

Dans ce paragraphe, nous énonçons pour les annérdes, sous certaines conditions, des propriétés connues (et que l'on retrouve comme cas particuliers) pour les anneaux. Plusieurs démonstrations seront omises car elles sont identiques à celles, classiques, du cas des anneaux ; on pourra se reporter à [6], chap. IV, $\S 4,5,6$. Nous noterons c.c.C. la condition de chaine croissante, c'est-à-dire la condition que toute chaıne strictement. croissante d'idéaux est finie.

Définition 1. Un idéal qui est intersection finie d'idéaux qui le contiennent strictement est dit réductible. Sinon, il est dit irréductible. Un idéal $\Im$ sera réductible si et seulement s'il existe deux idéaux $a$ et $b$ le contenant strictement, et tels que $\mathfrak{J}=a \cap b$. En effet, on peut toujours remplacer l'intersection finie par une intersection de deux idéaux contenant $\Im$ strictement.

Soit $p$ un idéal premier. Si on $a p=a \cap b, p$ contient $a b$, donc contient $\mathfrak{a}$ ou $\mathfrak{b}$ (III, $\S 3$, th. 1). Donc tout idéal premier est irréductible.

Lemme 1. Si un annérde vérifie la C.C.C。, tout idéal est intersection finie d'idéaux irréductibles.

Soit $\mathrm{F}$ la famille des idéaux de l'annérde qui ne sont pas intersection finie d'idéaux irréductibles. Si F est non vide, la c.c.c. montre que $\mathrm{F}$ possède un élément maximal $\Im_{0}$. Cet idéai est donc réductible, et il existe des idéaux $a$ et $\mathbf{b}$ contenant $\mathfrak{\Im}$ strictement et tels que $\mathfrak{J}=\mathbf{a} \cap \mathbf{b}$. Puisque $\mathfrak{Y}$ est maximal dans $F$, $\mathfrak{a}$ et $\boldsymbol{b}$ sont intersections finies $d^{\prime} i d e ́ a u x$ irréductibles, donc $\mathfrak{I}$ aussi, ce qui est contradictoire.

Lemme 2. Dans un annérde fort vérifiant la c.c.c., tout idéal irréductible est primaire.

Soit $q$ un idéal irréductible. Supposons-le non primaire. Il existe $b$ et $c \in A . . q$ tels que $b c \in q$ et $q u^{\circ}$ aucune puissance de $b$ n'appartienne à $q$. La suite des idéaux $q:\left\{b^{n}\right\}=\left\{z \in A ; z b^{n} \in q\right\}$ est croissante; la c.c.c. montre qu'elle est stationnaire à partir d'un entier s. D'autre part, A étant fort, $b$ est quasi-régulier pour $l$ 'indice $r$. Prenons alors $n=r+s$. Les idéaux $a=q+(c)$ et $b=q+A b^{n}$ contiennent $q$ strictement. Inversement, pour tout 
$x$ non nul dans $a \cap b$, il existe $u, v \in q, m \in \mathbb{Z}, \xi \in \overline{\mathrm{A}}^{\mathrm{b}}$ et $\eta \in \overline{\mathrm{A}}^{\mathrm{c}}$ tels que $x=u+\xi^{n}=v+\eta c+m c$. Puisque $x \neq 0$, tous les termes sont addibles à $x$ $(I, \S 1)$ donc entre eux. On obtient $b x=b v+\eta b c+m b c$, donc $b x \in q$ et aussi $\xi b^{n+1}=\left(\xi b^{n}\right) b=(x-u) b \in q$. Par quasi-régularité de $b, \xi$ est agglutiné par $b^{r}$ et on pose $d=\xi b^{r} \in A$. De $d b^{s+1}=\xi b^{n+1} \in q$ et de $q:\left\{b^{s+1}\right\}=q:\left\{b^{s}\right\}$ on déduit $\xi b^{n}=d b^{s} \in q$ et enfin $x \in q$. On aurait $q=a \cap b$ et $q$ serait réductible.

Définition 2. Lorsqu'un idéal a d'un annéride A peut s'écrire $a=\bigcap_{i=1}^{n} q_{i}$, ou $n \geq 1$ et ou les $q_{i}$ sont des idéaux primaires, on dit que $a$ possède une représentation ou décomposition primaire. Cette décomposition est dite réduite si aucun $q_{i}$ ne contient l'intersection des autres, et si les $q_{i}$ ont des idéaux premiers associés distincts. On peut toujours réduire une décomposition donnée (utiliser III, $\S 3$, th. 6 ).

Les lemmes 1 et 2 montrent ceci :

Théorème 1. Dans un annélde fort vérifiant la c.c.c., tout idéal admet une décomposition primaire réduite.

Remarque 1. En particulier, cette décomposition primaire existe dans un anneau vérifiant la c.c.c.. Elle existe aussi dans un annéfde A dont l'anneau $\bar{A}$ vérifie la c.c.c. : en effet, soit a un idéal de $A$, et $\bar{a}$ l'idéal de $\bar{A}$ agglutiné de a. Nous savons qu'une décomposition primaire se contracte (III, $\S 3$, théorème 8 et remarque 3 ). D'une décomposition réduite de $\bar{a}$ dans $\bar{A}$, on déduit par intersection avec $A$, une décomposition primaire de $a$, pas forcément réduite. Théorème 2. Dans un annérde $A$ dont I'anneau $\bar{A}$ vérifie la c.c.c., tout idéal admet une décomposition primaire réduite.

La décomposition primaire existe donc dans les annérdes qui sont noethériens forts, ou dont I'anneau est noethérien.

Théorème 3. Dans un annérde, soit a un idéal admettant une représentation primaire réduite $a=\bigcap_{i} q_{i}$. Pour que a soit son propre radical, il faut et il suffit que $q_{i}$ soit premier pour tout $i$.

Pour la démonstration, voir [6] p. 210.

Remarque 2. Si un idéal premier $p$ contient une intersection finie $n q_{i}$ d'idéaux, il contient l'un des $q_{i}$. Cela résulte de (III, $\S 3$, th. 1). Si, de plus, les $q_{i}$ sont primaires, $p$ contient le radical de l'un des $q_{i}$. Remarque 3. Si un idéal premier est une intersection finie d'idéaux premiers, il est égal à l'un d'eux. Cette décomposition primaire réduite d'un idéal a n'est pas forcement réduite $([6]$, p. 210$)$. Mais pour les idéaux premiers associés aux idéaux primaires de la décomposition, on a les théorèmes d'unicité suivants. 
Théorème 4. Dans un annétde $A$, soit un idéal a admettant une représentation primaire réduite $a=\eta_{i} q_{i}$ et notons $p_{i}=\sqrt{q_{i}}$. Pour qu'un idéal premier $p$ soit $l^{\prime}$ un des $p_{i}$, il faut et il suffit qu'il existe $c \in A, c \notin a$,

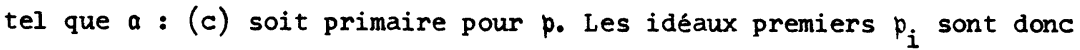
uniquement déterminés par $a$. Ils sont appelés idéaux premiers de $a$, ou associés à a.

La démonstration est classique (voir [6] p. 211).

Dans la famille des idéaux premiers de $a$, tout élément minimal est dit idéal premier isolé de $a$; un idéal premier de a non isolé est dit immergé. Dans une décomposition réduite de $a$, un idéal primaire (ou composante primaire) de a est dit isolé (resp. immergé) si son idéal premier l'est.

Théorème 5. Dans un annéfde $A$, soit a un idéal admettant une représentation réduite $a=\bigcap_{i} q_{i}$ et posons $p_{i}=\sqrt{q_{i}}$. Un idéal premier $p$ contient a si et seulement s'il contient l'un des $p_{i}$. Les idéaux premiers isolés de a sont les éléments minimaux de la famille des idéaux premiers contenant $a$.

Ceci résulte de la remarque 2.

Théorème 6. Dans un annéide $A$, soit a un idéal admettant une représentation réduite $a=\bigcap_{i} q_{i}$ et posons $p_{i}=\sqrt{q_{i}} \cdot$ L'ensemble $q_{i}^{\prime}$ des éléments $x$ de $A$ tels que $a:(x) \not \subset p_{i}$ est un idéal contenu dans $q_{i}$. Si $q_{i}$ est composante isolée de $a$, il est égal a $q_{i}^{\prime}$. Les composantes primaires isolées de $A$ sont donc uniquement déterminées par $a_{\text {. }}$

La démonstration est similaire à celle de [6], p. 212.

Remarque 4. Si $q_{i}$ est une composante primaire isolée d'un idéal $a$, et si $q$ contient $a$ et est un idéal $p_{i}$-primaire, $q$ contient $q_{i}$ (voir [5] p. 212).

Théorème 7. Soit $\mathrm{A}$ un annétde noethérien fort (resp. dont I'anneau $\bar{A}$ est noethérien) dont tout idéal premier propre est maximal. Alors, tout idéal a de $\mathbf{A}$ admet une unique décomposition primaire réduite. a est, de manière unique, un produit fini d'idéaux primaires dont les idéaux premiers sont distincts.

Dans un tel annéide, il n'y a pas de composante immergée. En effet, lorsque (0) est premier, il ne peut être idéal associé que de lui-même. La première assertion vient des théorèmes 1 et 2. La dernière utilise.(III, $\S 2 b$ ) et se montre comme dans [6] $\mathrm{p}, 213$.

Remarque 5. (Annérde quotient) soient $a$ et $b$ deux idéaux d'un annérde A; avec $a \supset b$. Pour $a$, etre premier (resp. primaire) s'exprime par une propriété de $A / \mathfrak{a}$ (III, $\S 3)$. Donc, pour que a soit premier (resp. primaire) il faut et il suffit que $a / b$ le soit (III, $\S 1$, théorème 4 bis d'isomorphisme). 
Comme $\sqrt{a / b}=\sqrt{a} / \mathfrak{b}$ (III, $\S 2 a,(3))$, à une décomposition réduite $a=\bigcap_{i} \mathfrak{a}_{i}$ de $a$ correspond la décomposition réduite $a / b=n_{i}\left(q_{i} / b\right)$ de $a / b$, et inversement; et $\mathfrak{p}_{i} / \mathfrak{b}$ est l'idéal premier associé à $q_{i} / \mathfrak{b}$. De plus, les composantes isolées (resp. immergées) se correspondent, car leur définition s'appuie sur des inclusions entre les idéaux premiers associés.

Théorème 8. Dans un annérde $A$, soit a un idéal admettant une décomposition primaire réduite $a=\prod_{i} q_{i}$. Alors, $\sqrt{a}$ est l'intersection des
idéaux premiers isolés de $a_{0}$

L'intersection étant finie, on a $\sqrt{\bigcap_{i} q_{i}}=\bigcap_{i} \sqrt{q_{i}}=\bigcap_{i} p_{i}$, et on enlève les idéaux premiers immergés.

Corollaire. Soit $A$ un annélde noethérien fort (resp. dont $\bar{A}$ est noethérien). L'ensemble $N$ des éléments nilpotents est l'intersection des idéaux premiers isolés de $(0)$ ( $c^{\prime}$ est-à-dire des idéaux premiers minimaux de $A$ ).

C'est clair, car $N=\sqrt{(0)}$. Notons que a est premier si et seulement $s^{\prime} i l$ possède une seule composante isolée.

Théorème 9. Soit $\mathrm{A}$ un annélde noethérien fort (resp. dont $\overline{\mathrm{A}}$ est noethérien). Soient $a$ et $b$ deux idéaux, $a \neq A$. Alors, $a=a: b$ si et seulement si $b n^{\prime}$ est contenu dans aucun idéal premier de $a$.

La démonstration est classique (voir [6] p. 214). On a les conséquences suivantes :

Corollaire 1. Soit $A$ un annérde noethórien fort (resp. dont $\bar{A}$ est noethérien). Pour qu'un idéal b soit contenu dans l'un des idéaux premiers associés à un idéal $a$, il faut et il suffit que $a \neq a: b$ 。

Corollaire 2. Soit $\mathrm{A}$ un annéíde noethérien fort (resp. dont $\overline{\mathrm{A}}$ est noethérien). Pour que $x \in A$ appartienne à l'un des idéaux premiers associés à un idéal $a$, il faut et il suffit qu'il existe $y \notin a$ tel que $x y \in a$.

On applique le corollaire 1 à $b=(x)$.

Corollaire 3. Dans un annérde A noethérien fort (resp. dont $\bar{A}$ est noethérien), l'ensemble $D$ de zéro et des diviseurs de zéro est la réunion des idéaux premiers de (0).

On applique le corollaire 2 à l'idéal $a=(0)$.

Corollaire 4 : Soient $\mathrm{A}$ un annérde dont I'anneau $\overline{\mathrm{A}}$ est noethérien, et $P$ l'ensemble formé de zéro et des diviseurs et pseudo-diviseurs de zéro de $A$. $P$ est 1 'intersection avec $A$ de la réunion des idéaux premiers de $\bar{A}$ associés à I'idéal $(0)$ de $\bar{A}$.

Cela résulte du corollaire 3.

Remarque 5. Toutes les définitions et propriétés ne faisant pas intervenir I'hypothèse que $A$ est fort et vérifie la c.c.c., sont vraies dans 
les anels (y compris celles où $\bar{A}$ vérifie la c.c.c.).

Définition 3. Pour un annérde $A$, on appelle zérorde de $A$ et on note $D$ l'ensemble formé de zéro et des diviseurs de zéro. On appelle pseudo-zéroide de $A$, et on note $P$ la réunion de $D$ et de l'ensemble des pseudo-diviseurs de zéro.

$D$ et $P$ sont stables par multiplication par les éléments de $A$, mais en général ne sont pas des idéaux.

Définition 4. Soit $A$ un annérde de graduation $\Delta$. On appelle idéal zéroide ou noyau zérorde de $A$ et on note $Z$ le noyau $\Delta$-large $Z=D_{L}$ de $D$, On appelle idéal pseudo-zérolde ou noyau pseudo-zérorde de A et on note w le noyau $\Delta$-large $W=P_{L}$ de $P$.

Lemme 3. Soient $\mathrm{A}$ un annérde de graduation $\Delta, \mathrm{D}$ et $\mathrm{P}$ respectivement son zéroide et son pseudo-zérolde. Pour tout $x \in P, x \notin D$, tel que $\omega(x)$ associe dans $\Delta$, I'enveloppe large de $x$ est contenue dans $P$. Si $\Delta$ est associative, l'enveloppe large de P...D est contenue dans $P_{n}$

Si $x$ est dans P..D, il existe $\alpha=\sum_{i=1}^{n} a_{i} \in \bar{A} . . A$, tel que $n \geqslant 2$ (les $a_{i}$ étant deux à deux non addibles, les $x a_{i}$ étant tous non nuls et deux à deux addibles), et tel que $x \alpha=0$. Pour tout multiple $\lambda \omega$ de $\omega=\omega(x)$, et pour tout élément $\mathrm{v}$ de $\mathrm{A}_{\lambda \omega}$, I'associativité par $\omega$ montre que, pour tout $v a_{i} \neq 0$, on $a: \omega\left(v a_{i}\right)=\omega(v) \omega\left(a_{i}\right)=(\lambda \omega) \omega\left(a_{i}\right)=\lambda\left[\omega \omega\left(a_{i}\right)\right]=\lambda \omega\left(x a_{i}\right)$. De même, pour tout $v \in A_{\omega}$ et pour tout $v a_{i} \neq 0$, on $a:$ $\omega\left(v a a_{i}\right)=\omega(v) \omega\left(a_{i}\right)=\omega(x) \omega\left(a_{i}\right)=\omega\left(x a_{i}\right)$. Dans les deux cas, $v$ agglutine $\alpha$ et on pose $\mathrm{b}=\mathrm{v} \alpha \in \mathrm{A}$. On a alors $\mathrm{b} \mathrm{x}=(\mathrm{v} \alpha) \mathrm{x}=\mathrm{v}(\alpha \mathrm{x})=\mathrm{v} 0=0$. Comme $\mathrm{x}$ ne divise pas zéro, $b=v \alpha$ est nul, et $v$ appartient à $P$. Donc I'idéal large engendré par $\{x\}$ est contenu dans $P$.

Lenme 4. Soit A un annélde sans diviseur de zéro. Munissons-le d'une graduation associative. L'idéal pseudo-zéroíde $W$ est égal au pseudo-zérolde $P$.

Par exemple, prenons la graduation propre. Le lemme 3 montre que $P$ est un idéal large.

\section{$\$ 2$ - INTERSECTION DES PUISSANCES D'UN IDEAL.}

L'anneau $\bar{A}$ est noethérien), et $a$ et $m$ deux idéaux de $A$. Alors il existe un
entier $s$ et un idéal $a^{\prime}$ tels que $m a=a n a^{\prime}$ et $a^{\prime} \supset m^{s}$.
Soit $\left\{q_{i}^{\prime}\right\}$ (resp。 $\left.\left\{q_{j}^{\prime \prime}\right\}\right)$ l'ensemble des composantes primaires de $m a$ dont l'idéal premier associé contient $m$ (resp. ne contient pas $m$ ). Posons 
$a^{\prime}=\bigcap_{i} q_{i}^{\prime}$ et $a^{\prime \prime}=\bigcap_{j} q^{\prime \prime}$. On a $m a=a^{\prime} \cap a^{\prime \prime}$, et il existe $s$ tel que $m^{s} \subset a^{\prime}$. Pour tout $j$, soit $m_{j} \in m, m_{j} \biguplus^{\prime} \sqrt{q_{j}^{\prime \prime}}$; pour tout $x \in a$, on a $m_{j} x \in m a \subset q_{j}^{\prime \prime}$, d'où $x \in q_{j}^{\prime \prime}$ et $a \subset a^{\prime \prime}$. On a finalement : $m a=m a \cap a=a^{\prime} \cap a^{\prime \prime} \cap a=a^{\prime} \cap a$.

Lemme 2. Soient a et $m$ deux idéaux d'un annérde unitaire A. Si $a=\mathfrak{m} a$, et si $a$ est non nul et de type fini, pour tout $x \in a^{*}=a_{0} .\{0\}$, Ion $\mathrm{a} x \in \mathrm{x}$.

Prenons un système fini $\left\{x_{1}=x, x_{2}, \ldots, x_{n}\right\}$ de générateurs de $a_{0}$ La relation $a=m a$ permet $d^{\mathfrak{c} e x p r i m e r}$ chaque $x_{i}$ par une combinaison linéaire sur $m$ des $x_{1}, \ldots, x_{n}$, d'où les $n$ équations :

$$
-\mu_{1}^{i} x_{1}-\ldots . .\left(\mu_{i}^{i}-1\right) x_{i}-\ldots . \mu_{j}^{i} x_{j}-\ldots . .=0
$$

où les $\mu_{j}^{i} \in \bar{m}$. Calculant dans $\bar{A}$, on montre que le déterminant des coefficients, soit $1-\mu$ avec $\mu \in \bar{m}$, est tel que $(1-\mu) x_{1}=(1-\mu) x_{1}=0$. Regroupons, dans le développement de $(1-\mu) x$, les termes addibles dans $A$; on obtient $(1-\alpha) x+\beta_{1} x+\ldots \ldots+\beta_{s} x=0$, où $x$ agglutine dans $A$ chacune des sommes formelles $\alpha, \beta_{1}, \ldots, \beta_{s} \in \bar{m}$. Comme $\bar{A}$ est somme directe des groupes d'addibilité de $A$, on a donc $(1-\alpha) x=0$, où $\alpha=\sum_{k} a_{k} \in \bar{m}$, et où $x$ et les $a_{k} x$ sont addibles.

Théorème 1. (de Krull) Soient A un annéride unitaire, et $m$ un idéal propre de A. Supposons que A soit noethérien fort ( $r \in s p$. que $\bar{A}$ soit noethérien). Pour que $n_{n}^{\infty} \mathfrak{m}^{n}=(0)$, il faut et il suffit que pour tout $x \neq 0$, on ait $x \notin x m$. $n=1$

Soit $x \neq 0$ tel que $x \in x m$. Il existe $\alpha=\sum_{i} a_{i}$ (les $a_{i} \in \mathfrak{m}$ ) tel que les $a_{i}$ soient non addibles deux à deux, Jes $a_{i} x$ et $x$ soient addibles deux à deux, et que $x=\alpha x=\sum_{i} a_{i} x$. En remplaçant chaque fois $x$ par la valeur précédente, on en déduit:

$x=\sum_{i} a_{i}\left(\sum_{j} a_{j} x\right)=\sum_{i} a_{i}\left[\sum_{j} a_{j}\left(\sum_{k} a_{k} x\right)\right]=\ldots \ldots \ldots \ldots$.

et il en résulte $x \in \mathfrak{m}^{n}$ pour tout $n$. Par suite, on a $\bigcap_{n=1}^{\infty} \mathfrak{m}^{n} \neq(0)$.

Posons $\mathfrak{J}=\bigcap^{\infty} \mathrm{m}^{\mathrm{n}}$. Lorsque $\mathfrak{J} \neq(0)$, le lemme 1 montre qu'il existe un entier s et un idéal $\mathfrak{J}^{\prime}$ tels que $m \mathfrak{I}=\mathfrak{I} \cap \mathfrak{J}^{\prime}$ avec $\mathfrak{J}^{\prime} \supset \boldsymbol{m}^{\mathrm{s}}$. On en déduit


montre que $x \in \mathrm{x}$.

Remarques sur le théorème de $\mathrm{Krull}$, et corollaires, en supposant que $A$ est un znnélde unitaire, noethérien fort (resp. dont l'anneau $\bar{A}$ est noethérien), de graduation fixée $\Delta$. On notera $D$ le zéroide (formé de 0 et des diviseurs de 0 ; il est la réunion des idéaux premiers de (0) dans A), $P$ le pseudo-zéroide (formé de $\mathrm{D}$ et des pseudo-diviseurs de 0), Z et W respecti- 
vement les idéaux zéroqde et pseudo-zérorde (noyaux larges de $D$ et $P$ ), $\mathfrak{m}$ un déal propre de $A, \mathfrak{m}_{L}$ son noyau large et enfin $\Im$ l'idéal $\Im=\prod_{n=1}^{\infty} \mathfrak{m}^{n}$. On suppose que $\omega(1)$ est unité de $\Delta$.

(0) - Cette remarque simple peut parfois alléger les conditions suffisantes des remarques qui suivent. Pour tout entier $s \geq 1$, on a $\omega\left(\mathfrak{m}^{s}\right) \subset \omega(\mathfrak{m})^{s}$. On en déduit les deux majorations :

$$
\begin{aligned}
& \omega\left(\bigcap_{n=1}^{\infty} m^{n}\right) \subset \bigcap_{n=1}^{\infty} \omega(\mathfrak{m})^{n} \subset \omega(\mathfrak{m})^{s} . \\
& \text { Dans les remarques }\left(3^{\prime}, 4^{\prime}, 5,5^{\prime}, 7\right) \text {, la famille } v \text { des éléments } v_{\omega}
\end{aligned}
$$

pourra donc être réduite, ou aux $\omega \in \omega(\mathbb{m})^{s}$ pour un $s$ fixé, ou aux $\omega \in \bigcap_{n} \omega(\mathbb{m})^{n}$. De plus, dans les remarques $\left(2^{\prime \prime}, 5^{\prime}, 10\right)$, l'intersection $\Im$ pourra parfois être serrée de plus près par ses grades.

Enfin, la condition $m \cap Z=(0)$ de la remarque (4') est vérifiée lorsque ( $s$ fixé $\geq 1): \omega(Z) \cap \omega(m)^{s}$ est formé de grades nuls.

(1) ISi (0) est le seul idéal contenu dans $m \cap P$, il en résulte $\Im=(0)$.

En effet, si cette intersection $\Im \neq(0)$, tout $x \in \Im, x \neq 0$, est diviseur ou pseudo-diviseur de 0 .

(19) Si m n'a pas de pseudo-diviseur propre de zéro, pour que $\Im=(0)$, il faut let il suffit que 1 - $m n^{\prime}$ ait pas de diviseur de zéro.

C'est une conséquence immédiate du théorème.

(2) - Un element $x \in A$ sera dit 1-régulier si $x \neq 0$ et si pour tout $a \in A$, $0 \neq x a \# x$ implique a \#1. Un élément $\xi \in \Delta$ sera dit 1 -régulier si $\xi \neq 0$ et si, pour tout $\alpha \in \Delta$, la relation $\xi \alpha=\xi$ implique $\alpha=\omega(1)$. Un élément qui $n$ 'est pas 1-régulier est dit 1-irrégulier. Si $\xi$ est 1-régulier, les éléments non nuls de $A_{\xi}$ sont 1-réguliers. Notons $\Gamma$ l'ensemble des $\xi \in \Delta$ qui sont 1-irréguliers, et notons $G=A_{\Gamma}$ la réunion des groupes d'addibilité associés. Tous les éléments 1-irréguliers de $A$ sont contenus dans $G$. Lorsque $\Delta$ est associative, $\Gamma$ est un $\Delta$-idéal, et $G$ un idéal large. En effet, soit $\xi \in \Gamma, \xi \neq 0$; il existe $\alpha$ non nul différent de $\omega(1)$ tel que $\xi \alpha=\xi$, et pour tout $\lambda \in \Delta$, on a $\lambda \xi=\lambda(\xi \alpha)=(\lambda \xi) \alpha$, d'ou $\lambda \xi \in \Gamma$.

Lorsque 1 - $\mathfrak{m}$ est sans diviseur de zéro :

a) $x$ 1-régulier implique $x \notin \Im$

b) $\xi$ 1-régulier implique $\mathfrak{I}_{\xi}=\Im \cap A_{\xi}=(0)$

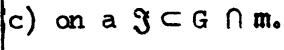

En effet, si $x \in \Im$ est 1-régulier (donc $x \neq 0$ ), il existe $\alpha \in \bar{m}$ tel que $x(1-\alpha)=0$. Puisque $x$ est $1-$ régulier, $1-\alpha$ est élément de $A$, donc $1-\alpha \in 1-m$ divise 0 , une contradiction.

De (2) et du théorème de Krull, il résulte : 
(2') $\mid$ Si les éléments de $\mathfrak{m}^{*}=\mathfrak{m}_{.} .\{0\}$ sont 1-réguliers, pour que $\Im=(0)$ il faut et il suffit que 1 - $m n^{\prime}$ ait pas de diviseur de zéro (c'est-à-dire que $\mathfrak{m}+p_{i} \neq$ A pour tout idéal premier $p_{i}$ de $(0)$ dans A).

Il résulte de (2) :

(2") Soit $\mathrm{K}$ un idéal de $\mathrm{A}$. Si les éléments de $m_{.} . \mathrm{K}$ sont 1-réguliers (par exemple si ceux de $\omega\left(m_{0} . K\right)$ le sont), et si $1-m^{\prime}$ a pas de diviseur de zéro, on a $\Im \subset \mathfrak{m} \cap \mathrm{K}$.

(3) Pour un élément $\omega \in \omega\left(\mathfrak{m}^{*}\right)$ (avec $\mathfrak{m}^{*}=\mathfrak{m}_{0} .\{0\}$ ), supposons qu'il existe $v_{\omega} \in A_{\omega}^{*}=A_{\omega} .\{0\}$ ne divisant pas zéro. Si $v_{\omega}-v_{\omega} m$ et $D$ sont disjoints, on a $3_{\omega}=(0)$.

Sinon, il existe $x$ non nul dans $A_{\omega}$ et $\alpha=\sum_{i} a_{i} \in \bar{m}$ tels que $x(1-\alpha)=0\left(\right.$ avec $x a_{i} \neq 0$ pour tout $\left.i\right)$. Mais $v=v_{\omega}$ agglutine $1-\alpha ;$ posons $\mathrm{b}=\mathrm{v}(1-\alpha)$. Puisque $\mathrm{b} x=v(1-\alpha) \mathrm{x}=\mathrm{v} 0=0$, b est nul ou divise zéro, une contradiction.

(3') ISupposons que, pour tout $\omega \in \omega\left(\mathrm{m}^{*}\right)$, il existe un non diviseur de zéro $v_{\omega} \in A_{\omega}^{*}$, et notons $v$ une famille $\left\{v_{\omega}\right\}_{\omega \in \omega\left(n_{1}{ }^{*}\right)}$ de tels éléments (dite famille d'agglutinateurs associée à $m$ ). Pour que $\Im=(0)$, il faut et il suffit que pour tout $v \in v, v-v m$ soit disjoint de $D$ (c'est-à-dire que $v \notin \mathbf{v}+p_{i}$, pour tout idéal premier $p_{i}$ de $\left.(0)\right)$.

Il reste à montrer que la condition est nécessaire. S'il existe $v \in V$ tel que $v-v m$ rencontre $D$, il existe $\alpha \in \bar{m}$ tel que $1-\alpha \in \vec{A}^{v}$ et que $b=v(1-\alpha) \in D$. Ou $b=0$ et on $a v \in v$ m. Ou b divise zéro, soit $b d=0$ et on $a$ : $v d \neq 0$ et $v d \in v d m$. Dans les deux cas, $\Im$ contient un élément non nul. Lorsque de plus $\omega(m)$ est fini, $V$ aussi, et on peut se borner à vérifier la condition $(u-u m) \cap D=\varnothing$ pour le produit $u$ des $v_{\omega}$, car $u$ ne divise pas zéro et agglutine de même toute somme formelle 1- $\alpha$ considérée plus haut. Il en est de même si les $v_{\omega}$ ont un multiple commun non nul et non diviseur de zéro. (4) Soit $\omega$ un élément fixé de $\omega\left(m^{*}\right)$. Supposons que $w$ associe dans $\Delta$, et qu'il existe un multiple $\xi=\lambda \omega$ de $\omega$ et un non-diviseur de zéro $v_{\omega} \in A_{\xi}$ tels que $v_{\omega}{ }^{-v} \omega^{m}$ et $D$ soient disjoints. Alors on a $\mathfrak{S}_{w}=(0)$ :

Sinon, de meme qu'au (3), notons $x$ non nul dans $\jmath_{\omega}$ et $\alpha \in \bar{m}$ tels que $x(1-\alpha)$ (avec $x a_{i} \neq 0$ pour tout $i$ ). Pour tout multiple $\xi=\lambda \omega$ de $\omega$ et tout non-diviseur de zéro $v_{\omega} \in A_{\xi}, I$ associativité par $\omega$ montre que : $\xi \omega\left(a_{i}\right)=(\lambda \omega) \omega\left(a_{i}\right)=\lambda\left[\omega \omega\left(a_{i}\right)\right]=\lambda \omega\left(x a_{i}\right)=\lambda \omega$, donc que $\xi$ agglutine 1- $\alpha$. Alors, $v_{\omega}$ agglutine $1-\alpha$, et $b=v_{\omega}(1-\alpha)$ est nul ou divise zéro, une contradiction. Un tel élément $v_{\omega}$ est dit agglutinateur (a)-associé à w. 
(4') Plus généralement, si les éléments de $\omega(m)$ associent dans $\Delta$, et si pour tout $\omega \in \omega\left(m^{*}\right)$, il existe un multiple $\lambda \omega$ et un non-diviseur de zéro $v_{\omega} \in A_{\lambda \omega}$, notons $V$ une famille de tels agglutinateurs, (a)-associée à m. Pour que $\Im=(0)$, il faut et il suffit que, pour tout $v \in V, v-v m$ et $D$ soient disjoints. (Donc que $\mathbf{v} \notin \mathbf{v}+p_{i}$, pour tout idéal premier $p_{i}$ de $\left.(0)\right)$.

Cela résulte de (4) et de la démonstration de ( $\left.3^{\prime}\right)$. Le choix de la famille $V$ est ici plus large qu'en ( $\left.3^{\prime}\right)$. De plus, si les éléments de $w\left(\mathrm{~m}^{*}\right)$ ont un multiple commun $\xi$, er $s^{\prime} i l$ existe un non-diviseur de zéro $v \in^{A_{\xi}}$, on prendra $\mathrm{V}=\{\mathrm{v}\}$.

Lorsque $\Delta$ est associatif, la définition de l'idéal zéroide $\mathrm{Z}$ de $\mathrm{A}$ montre que l'existence de $\mathrm{V}$ équivaut à la condition $\mathrm{m} \cap \mathrm{Z}=(0)$, donc :

(5) Si $\Delta$ est associative, et si $\mathrm{m} \cap \mathrm{Z}=(0)$, soit $\mathrm{V}$ une famille d'agglutinateurs (a)-associée a $m$. Pour que $\mathfrak{S}=(0)$, il faut et il suffit que, pour tout $v \in v$ et pour tout idéal premier $p_{i}$ de $(0)$, on ait $v \notin v \mathbf{m}+\mathfrak{p}_{\mathbf{i}}$.

(5') Soit $K$ un idéal de $A$. On suppose que $\Delta$ est associative, et que (m..K) $\cap \mathrm{Z}=\varnothing$. Soit $V=\left\{v_{\omega}\right\}_{\omega \in \omega\left(m_{.} . K\right)}$ une famille d'agglutinateurs, (a)-associée à m...K. Si pour tout $v \in v$ et pour tout idéal premier $p_{i}$ de $(0)$ on a $v \notin v m+p_{i}$, il en résulte $\Im \subset \mathfrak{m} \cap \mathrm{K}$.

Cela résulte de (4).

(5) |Lorsque $\Delta$ est associative, on a : $\Im \subset(D \cup W) \cap \mathbf{m} \cap \mathrm{G}$.

Cela résulte de (2), et de ce que, si un pseudo-diviseur propre de

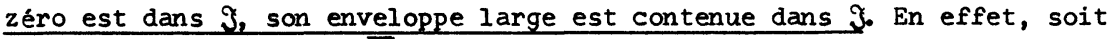

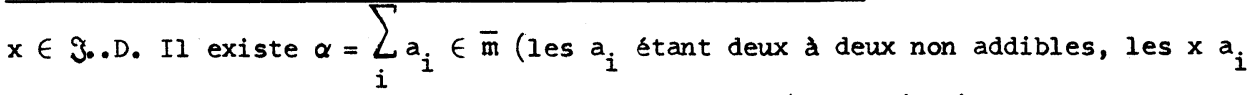
étant tous non nuls et addibles à $x)$ tels que $1-\alpha \notin A$ et $x(1-\alpha)=0$. Tout multiple $\lambda \omega$ de $\omega=\omega(x)$ agglutine $1-\alpha$, puisque $\Delta$ est associative : $(\lambda \omega) \omega\left(a_{i}\right)=\lambda\left[\omega \omega\left(a_{i}\right)\right]=\lambda \omega\left(x a_{i}\right)=\lambda \omega$. Il en résulte que tout $c$ non nul appartenant à $A_{\lambda \omega}$ agglutine aussi $1-\alpha$; on en déduit $b=c(1-\alpha) \in A$ et $b x=0$. Comme $x \neq 0$ ne divise pas zéro, b est nul. Alors, $c=c \alpha$ montre que $c$ est dans $\Im$. Donc 1 'enveloppe large de $\{x\}$ est contenue dans $\mathfrak{J}$.

Un annélde unitaire est dit quasi-domaine $s^{\prime} i l n^{\prime} a$ pas de diviseur de zéro; alors sa graduation peut etre choisie associative. Il est dit domaine si, de plus, il n'a pas de pseudo-diviseur de zéro (II, $\S 1$ ). On en déduit de (5): (7) Soient A un quasi-domaine, $\Delta$ une graduation associative de A, et V une famille (a)-associée a m. Pour que $S=(0)$, il faut et il suffit que pour tout $v \in v$, on ait $v \notin v$ m.

Dans ce cas, $D=(0)$ et $\mathrm{V}$ existe. $D^{\prime}$ autre part, la démonstration de 
(6) montre que, dans un quasi-domaine, I est large. On en déduit :

(7') Soient $A$ un quasi-domaine, et $\Delta$ une graduation associative de A. Alors, $\Im$ fest un idéal large contenu dans $w \cap m_{L} \cap G$.

(8) ISi A est un domaine, on a $S=(0)$.

Cela résulte de (1).

(9) Si $\Delta$ est associative et si $\mathfrak{m}$ est un idéal maximal, tout élément de $3_{0 .} m_{L}$ est un diviseur de zéro. Si, en particulier, 1-m $n^{\prime}$ a pas de diviseur de 0 , fon a $3 \subset m_{L}$.

Pour tout $x \in \Im .0 m_{L}$ il existe $\alpha=\sum_{i} a_{i} \in \bar{m}$ tel que $x(1-\alpha)=0$

(avec les $a_{i}$ deux à deux non addibles, et les $x a_{i}$ tous non nuls et addibles à $x$ ). Puisque $x \notin \mathfrak{m}_{L}$ il existe un multiple $\lambda \omega$ de $\omega=\omega(x)$ et il existe un élément $c$ de $A_{\lambda \omega}$ tel que $c \notin m$. Comme $A$ est unitaire, $\Delta$ associative et $m$ maximal, $A / m$ est un corpolde ; donc $c$ possède un inverse modulo $\mathfrak{m}:$ il existe $a \in \mathfrak{m}$ et $c^{\prime} \notin \mathfrak{m}$ tels que $c^{\prime} c=1+a$. Le grade de $c^{\prime} c$ est donc $l^{\prime}$ unité $\omega(1)$ de $\Delta$, et $\Delta$ associatif implique

$$
\omega(1)=\omega\left(c^{\prime} c\right)=\omega\left(c^{\prime}\right) \omega(c)=\omega\left(c^{\prime}\right)(\lambda \omega)=\left[\omega\left(c^{\prime}\right) \lambda\right] \omega=\lambda^{\prime} \omega
$$

en posant $\lambda^{\prime}=\omega\left(c^{\prime}\right) \lambda$. L'associativité de $\Delta$ montre enfin, pour tout $i$, que $\omega\left(a_{i}\right)=\omega(1) \omega\left(a_{i}\right)=\left(\lambda^{\prime} \omega\right) \omega\left(a_{i}\right)=\lambda^{\prime}\left[\omega \omega\left(a_{i}\right)\right]=\lambda^{\prime} \omega\left(x a_{i}\right)=\lambda^{\prime} \omega=\omega(1)$. Par suite, 1- $\alpha$ est un élément de $A$ et $x$ divise zéro.

(10) - L'annérde unitaire A noethérien fort (resp. dont $\bar{A}$ est noethérien) sera dit local si et seulement si il possède un seul idéal maximal $m$, c'est-à-dire si l'ensemble des éléments de $\mathrm{A}$ qui sont ni inversibles, ni pseudo-inversibles est un idéal $\mathbf{m}$. L'hypothèse locale sert peu pour appliquer la remarque (4), sauf si une famille $\mathrm{V}$ est formée d'inversibles et pseudo-inversibles, car on peut la remplacer par $\{1\}$; alors $\Im=(0)$ car $1-\mathfrak{m}$ est sans diviseur de zéro. Si A est local, à graduation associative, on a $\Im \subset V \cap G$.

Cela résulte des remarques (9) et (6), car 1-m est sans diviseur de zéro.

Exemple contraire : Soient $k$ un corps, et $s$ un entier $\geqslant 2$. Soit 1 'anneau $\bar{R}=k[x, y, z] /\left(x^{s}-x\right)$ des polynomes sur $k$ à trois variables $x, y, z$ liées par la relation $x^{s}=x_{0} L^{\prime}$ annérde $R$ des monômes de $\bar{R}$ est noethérien fort, et son idéal $(x)=p$ est premier (d'ailleurs strictement). Notons $A=R_{p}$ l'annérde local des monômes a $x^{m} y^{n} z^{p}$, avec a $\in k, m \in\{c, \ldots, s-1\}$, n et $p \in \mathbb{Z}$. $\mathfrak{m}=\mathrm{A} \times$ est $\mathrm{l}$ 'unique idéal maxinal de $A$, et les élénents de A..m sont inversibles. Enfin A est noethérien fort.

D'une part, $\cap^{n} m^{n}=m$, puisque $x=x^{s}$.

D'autre part, l'idéal $\overline{\mathfrak{m}}$ de $\overline{\mathrm{A}} \mathrm{n}$ 'est pas maximal, puisque

$\overline{\mathrm{m}}=\overline{\mathrm{A}} \mathrm{x} \neq \overline{\mathrm{m}}+\overline{\mathrm{A}}(\mathrm{y}+\mathrm{z}) \neq \overline{\mathrm{A}}$. 
Enfin, l'idéal pseudo-zérolde de $A$ est $\mathbf{m}$.

( $L$ 'idéal zéroìde, et nêne l'ensemble $D$ des diviseurs de zéro, sont réduits à $(0)$ ).

Théorème 2. Soient $A$ un annêtde unitaire noethérien fort (resp. dont I'anneau $\overline{\mathrm{A}}$ est noethérien) et $\boldsymbol{m}$ et a deux idéaux própres de A. Notons $p_{1}, \ldots \ldots p_{s}$ les idéaux premiers de $a_{0}$ On suppose vérifiée $l^{\circ}$ une des trois hypothèses suivantes :

$(1 \mathrm{r})$ - Les éléments de m. 。 a sont 1-réguliers et on pose $V=\{1\}$ 。

$(n)$ - Pour tout $\omega \in \omega\left(m_{.} . a\right)$, i.l existe $v_{\omega} \in A_{\omega}$ tel que $v_{\omega} \notin \underset{i}{U} p_{i}$ et on note $V=\left\{v_{\omega}\right\}_{\omega \in \omega\left(m_{0} \circ a\right)}$ une famille de tels éléments, associée à m.. $a_{0}$

(a) - $\Delta$ est associative, et on $a . H \cap m \subset a$, où $I^{\prime}$ on note $H I^{\prime} i d e ́ a l$ contenu dans $\bigcup_{I} p_{i}$, et tel que $H / a$ soit I'idéal zéroíde $Z^{\prime}$ de $A / a^{*}$ Enfin, on note $V$ une famille d'agglutinateurs $v_{\omega} \notin \mathrm{U}_{i},(a)$-associée à m.•a.

Alors, pour que $\bigcap_{n=1}^{\infty}\left(a+m^{n}\right)=a$, il faut et il suffit que, pour

tout $v \in V$ et pour tout $i=1, \ldots, s$, on ait $v \notin v m+p_{i}$ 。

Dans le cas $(1 r)$, la condition s'écrit $m+p_{i} \neq A$ pour tout

$i=1, \ldots, s$.

Posons $A^{\prime}=A / a, \mu=(m+a) / a$ et pour tout $a \in A$, notons $a^{\prime}=\varphi(a)$

sa classe modulo a. La condition $n=1\left(a+m^{n}\right)=a$ équivaut à $\bigcap_{n}^{\infty}\left(a+m^{n}\right) / a=(0)$, donc à $\bigcap_{n=1}^{\wp} \mu^{n}=(0)$. (III, $\S 2 a$, formule (11))

Dans le cas ( $1 \mathrm{r})$, la 1-régularité passe au quotient : tout $\mathrm{x}^{\prime} \in \mu$, $\mathbf{x}^{\prime} \neq 0$, et tout $a^{\prime} \in A^{\prime}$ sont classes modulo a respectivement $d^{\prime} u n x \in m_{0}$. $\mathbf{a}$ et d'un $a \in A$. Si $I^{\prime}$ on a $0 \neq x^{\prime} a^{\prime} \# x^{\prime} \neq 0$, c'est-à-dire $0 \neq \varphi(x a) \# \varphi(x) \neq 0$, cela implique $x a \# x$ car $\varphi$ est un homomorphisme. Mais $x$ étant 1-régulier, on déduit a \#1, donc aussi $a^{\prime} \# 1^{\prime}$.

Dans le cas $(n)$, l'existence d'un $v_{\omega} \in A_{\omega}$ tel que $v_{\omega} \notin \bigcup_{i} p_{i}$ équivaut à $I^{\prime}$ existence dans $I^{\prime}$ annélde quotient $A^{\prime}$ d'un non diviseur de zéro $v_{\omega}^{\prime}=\varphi\left(v_{\omega}\right) \in A_{\omega}^{\prime}$.

Notons $D^{\prime}$ le zéroide et $Z^{\prime} I^{\prime}$ idéal zéroide de $I^{\prime}$ annérde $A^{\prime}=A / a^{\circ}$

Nous savons que $D^{\prime}$ est la réunion des idéaux $\mathfrak{p}_{i / a^{*}}$ Dans le cas (a), la condition $\mathrm{H} \cap \mathfrak{m} \subset \boldsymbol{a}$ implique, pour tout $\omega \in \omega\left(\mathbf{m}_{0} . \mathfrak{a}\right)$, l'existence d'un multiple $\lambda \omega$ et d'un agglutinateur (voir remarques (4) à (5')) $v_{\omega} \in A_{\lambda \omega}$ tel que $v_{\omega} \notin \cup p_{i}$. Cette condition équivaut à $Z^{\prime} \cap \mu=(0)$, c'est-à-dire implique, pour tout $\omega \in \omega\left(\mu^{*}\right)$, I'existence $d^{\prime} u n$ agglutinateur $v_{\omega}^{\prime} \in A_{\lambda}{ }_{\omega}$. La caractérisation de l'idéal $\mathrm{H}$ est noins simple que celle d'un idéal zéroide. On a : $a \subset H \subset \bigcup_{i} \mathfrak{p}_{i^{\circ}}$ L'idéal $H$ est large sur tout grade où il excède $a: i l$ est le 
plus grand idéal vérifiant en outre la condition : pour tout $\delta \in \Delta$ ou $H$ excède $\mathfrak{a}$, pour tout multiple $\lambda \delta$ de $\delta$, l'idéal $H$ est large sur $\lambda \delta$. On peut dire que $H$ est large dans son excès sur a (on sait que $\mathrm{H}$ n'est pas forcément large; voir III, §5).

Notons enfin, puisque $p_{i}$ contient $a, q u e v \notin v m+p_{i}$ équivaut à $\varphi(v)=v^{\prime} \notin v^{\prime} \mu+\left(p_{i / a}\right)$. Les résultats s'obtiennent alors respectivement par les remarques $\left(2^{\prime}\right),\left(3^{\prime}\right),\left(4^{\prime}\right)$.

Cas particuliers - (1) Si a est strictement premier, pour tout idéal propre $\mathfrak{m}$, on a $\cap\left(\mathfrak{a}+\mathfrak{m}^{n}\right)=a_{\text {o }}$ En effet, on applique (8) au domaine $A / a^{*}$

(2) Si a est premier, $(n)$ est trivialement vérifiée. De plus, bien que (a) ne soit pas forcément vérifiée (associativité de $\Delta$ ), $A / a$ est un quasidomaine et possède une graduation associative $\Delta^{\prime}$. Alors, grâce à la remarque (7), il existe dans $\mathrm{A} / \mathrm{a}$ une famille d'agglutinateurs (a)-associée à $\mu$, et on peut en déduire dans $\mathrm{A}$ une famille $\mathrm{V}$ d'agglutinateurs (a)-associée à m.. a. Ceci étant, la condition $v \notin v m+a$ est nécessaire et suffisante pour que $n\left(a+m^{n}\right)=a_{0}$

(3) Si A est local, si $\Delta$ est associative, et si l'idéal maximal m est étroit, pour tout idéal propre $a$, on a ${ }_{n}\left(a+\mathfrak{m}^{n}\right)=a$.

$\mathrm{m}$ étant étroit, il existe une famille $\mathrm{V}$ d'agglutinateurs (a)-associée à $\mathfrak{m}$, formée d'inversibles et de pseudo-inversibles, donc remplaçable par \{1\}. Pour tout idéal propre a de $A$, et pour tout idéal premier $p_{i}$ de $a, p_{i} \subset \mathfrak{m}$ implique $1 \notin \mathfrak{m}+\mathfrak{p}_{i}$, d'où le résultat.

Théorème 3. Soit $A$ un annéide unitaire noethérien fort (resp. dont $\bar{A}$ est noethérien), et soient $\boldsymbol{m}, \boldsymbol{K}$ et a trois idéaux propres de A. Notons $p_{i}, i=1,2, \ldots, s$ les idéaux premiers de a. On fait l'une des trois hypothèses suivantes : $(1 \mathrm{r}$ ) Les éléments de m..K sont 1-réguliers. On pose $V=\{1\}$. $(n) K$ est faiblement large. Pour tout $\omega \in \omega\left[\mathfrak{m}_{\bullet} .(K+a)\right]$, il existe $v_{\omega} \in A_{\omega}$ tel que $v_{\omega} \notin \bigcup_{i} p_{i}$. On note $V$ une famille de tels éléments, associée à $m_{0} .(K+a)$. (a) $\Delta$ est associatif et $\mathbb{K}$ est faiblement large. $Z^{\prime}=H / a$ étant $I^{\prime}$ idéal zéroide de $A / \mathfrak{a}$, on a $H \cap \mathfrak{m} \subset \mathfrak{a}+K$, et on note $V$ une famille d'éléments $v_{\omega} \notin \bigcup_{i} p_{i}$, (a)-associée à mo. $(a+K)$.

Alors, si pour tout $v \in v$, et pour tout $i=1, \ldots, s$, on $a$

$v \notin v m+p_{i}$, cela implique $\bigcap_{n=1}^{\infty}\left(a+m^{n}\right) \subset a+(K \cap \mathfrak{m})$. 
Si $K$ est faiblement large, on a $a+K=a \cup K$. Pour la démonstration, on passe au quotient $\mathrm{A} / \mathrm{a}$ auquel on applique les remarques (2"), (3) et (5') respectivement. Noter que la condition suffisante, dans le cas ( $1 \mathrm{r}$ ) est que $m+p_{i} \neq A$ pour tout i。 Corollaire 1 . Avec les hypothèses du théorème 3 , on a
$n_{n=1}^{\infty}\left(a+m^{n}\right) \subset \prod_{j=1}^{r} q_{j}+(k \cap m)$ où les $q_{j}$ sont les composantes primaires de $a$
dont le radical $\mathfrak{p}_{j}$ vérifie la condition $v \notin \mathrm{vm}+\mathfrak{p}_{j}$ pour tout $v \in \mathrm{v}$.
En effet, $a \subset \cap q_{j}$ et on applique à ce dernier idéal le théorème.
Corollaire 2. Soit A un annéide unitaire noethérien fort (resp. dont $\bar{A}$ est noethérien). Si A est local d'idéal maximal $m$, pour tout idéal $a$ de $A$, on a $\bigcap_{n=1}^{\infty}\left(a+m^{n}\right) \subset a+m_{L}$.

En effet, les éléments de $\mathrm{m}_{\text {... }} \mathrm{m}_{\mathrm{L}}$ sont 1-réguliers, par définition du noyau large $m_{L}$ de $m$ et parce que $A$ est local. Tout idéal premier, étant contenu dans $\mathrm{m}$, ne peut lui être comaximal; et le corollaire 1 montre le résultat.

Soient un annérde unitaire A noethérien fort (resp. dont l'anneau $\bar{A}$ est noethérien), et $m$ un idéal de $A$. Lorsqu'on prend pour voisinages fondamentaux de $x \in A$ la famille $U_{n}(x)$ des classes de $x$ modulo $m^{n}$ (attention : $U_{n}(x)=x+m^{n}$ seulement si $x=0$ ou $\left.x \notin m^{n}\right)$, on vérifie aisément que cela définit une topologie sur A, qui fait de $\mathrm{A}$ un annérde topologique (là interviennent les lois quotient dans $\left.A / m^{n}\right)$. Lorsque $\bigcap_{n=1}^{n} m^{n}=(0)$, A est un espace topologique séparé; alors, la condition $n\left(a+m^{n}\right)=a d u$ théorème 2 signifie que $I^{\prime}$ idéal a est fermé pour cette topologie; si $\left\{a_{i}\right\}_{i \in I}$ est une famille d'idéaux, fermés dans la topologie relative à $m$, il est clair que leur intersection aussi est un idéal fermé. Noter que tout idéal strictement premier a de A est fermé dans cette topologie (cas particulier (1) du théorème 2). Noter aussi que, dans les conditions du corollaire 2, si m est étroit, c'est-à-dire si $m_{L}=(0)$, tout idéal de $A$ est fermé dans cette topologie (cas particulier (3) du théorème 2).

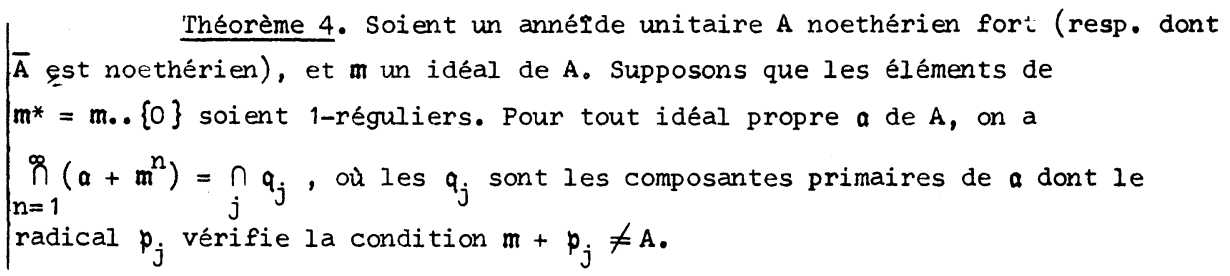


Soit $b=\bigcap_{j} q_{j} \cdot$ Par le théorème $2(1 r)$, chaque $q_{j}$ est fermé, donc aussi leur intersection $b=n\left(b+m^{n}\right)$. Soi.ent $q_{i}^{\prime}(i \in I)$ les autres composantes


$\S 2 b)$ avec chaque $p_{i}^{\prime}$, donc avec chaque $q_{i}^{\prime}$, donc avec leur intersection $b^{\prime}=\eta q_{i}^{\prime}$. Enfin, pour tout $n \geq 1$, $b^{\prime}$ et $m^{n}$ sont comaximaux. On a donc : $b=A \quad b=\left(b^{\prime}+m^{n}\right) b=b^{\prime} b+m^{n} b c a+m^{n}$. On en déduit $: b+m^{n}=a+m^{n}$, d'où enfin $\cap\left(a+m^{n}\right)=b$.

$n$

\section{$\$ 3$ - ANNEIDES DE FRACTIONS.}

Un intégroìde $R$ est un annérde commutatif où tout élément non nul est simplifiable (II, $\S 1$ ); $R n^{\prime}$ a pas de diviseur de 0 , mais peut avoir des pseudo-diviseurs de 0 .

Lemme 1. Soient $R$ un intégroìde et $M$ une partie multiplicative de $M$ (avec $0 \notin M$ ). Le quasi-morphisme $h: R \rightarrow R_{M}$ est injectif, et $R_{M}$ est un intégroîde.

En effet, pour tous $a, b \in R$, la relation $h(a)=h(b)$ équivaut à l'existence de $m \in M$ tel que $m a=m b$; on obtient $a=b$ en simplifiant.

Ceci nous permet d'identifier $R$ à la partie $h(R)$ de $R_{M}$. Mais $h(R)$ $n$ 'est pas forcément stable pour l'addition, car l'addibilité des éléments de $h(\mathbb{R})$ est en général plus large que celle des éléments correspondants de $\mathbf{R}$ (ce "plongement" est similaire à l'inclusion d'un annérde dans un annérde agglutiné).

Soient $\frac{a}{s}, \frac{b}{t}$ et $\frac{c}{u} \in R_{M}$ vérifiant les conditions $\frac{c}{u} \neq 0$ et $\frac{c a}{u s}=\frac{c b}{u t}$. Il existe $m \in M$ tel que $m u t c a=m u s c b$. En simplifiant par $c$, on obtient $(m u) t a=(m u) s b$, d'où $\frac{a}{s}=\frac{b}{t}$. Donc $R_{M}$ est un intégrớde.

En particulier, si $R$ est un intégroíde et si $R^{*}=R_{0} .\{0\}$, ce lemme permet de plonger, au sens précédent, tout annérde $R_{M}$ de fractions dans $I^{\prime}$ annéride $A=R_{R^{*}}$. En effet, soit $h$ le quasi-morphisme $: R \rightarrow R_{M^{*}}$ Puisque $R_{M}$ est un intégroìde, il se plonge dans $\left(R_{M}\right)_{h\left(R^{*}\right)}$. Or, ce dermier est isomorphe à $A$ (III, $\S 4 \mathrm{~b}$, remarque 3 ).

Théorème 1. Supposons que l'annélde unitaire $\mathrm{R}$ soit un intégrorde. Soit $E$ l'ensemble de ses idéaux maximaux. Pour tout idéal $b$ de $R$, on $a$ : $\mathfrak{b}=\bigcap_{\mathfrak{m} \in E} b_{\mathbf{m}_{\mathfrak{m}}}$. On a en particulier $\mathrm{R}=\bigcap_{\mathfrak{m} \in E} R_{\mathfrak{m}}$.

$R_{m}$ désigne I'annérde de fractions relatif à $m$ (III, $\S 4 c$, exemple (1)). On note $b R_{m}$ l'extension de $b$ par $R \rightarrow R_{m}$, et on considère chaque annérde $R_{m}$ comme plongé, au sens précédent, dans $A=R_{R^{*}}$ 
Lemme 2. $R$ vérifiant les conditions du theorème, soit $\frac{a}{s}=\frac{b}{t}$ une fraction non nulle de $R_{R} *=A$, telle que $s$ et $t$ soient addibles a $A$. Alors, $a$ et $b$ sont addibles, et $s i s+t \neq 0$, on $a \frac{a}{s}=\frac{a+b}{s+t}$.

$\mathrm{R}$ étant un intégroide, $\frac{a}{s}=\frac{b}{t} \neq 0$ équivaut à ta $=s b \neq 0$. De $\mathrm{s} \# 1$ \#t, on déduit $\mathrm{b} \# \mathrm{sb}$ et ta \#a. Par transitivité, il en résulte $\mathrm{a} \# \mathrm{~b}$. La seconde assertion est alors triviale.

L'inclusion $b \subset \cap_{\mathfrak{m}} b R_{M}$ est évidente. Considérons un élément $x$ non nul du second membre. Pour tout $m \in E$, la fraction $x$ peut s'écrire $\frac{b_{m}}{s_{m}}$ avec $b_{\mathfrak{m}} \in b$ et $s_{\mathfrak{m}} \notin \mathfrak{m}$ (III, $\S 4 c$, lemme 6). L'idéal $\mathfrak{J}$ engendré par les $s_{\mathfrak{m}} n^{\prime}$ est contenu dans aucun idéal maximäl. Donc $\Im$ est égal à A (III, $\S 3$, remarque 1), et 1 est combinaison linéaire des $s_{m}$ On a donc :
$1=\sum_{m \in F}\left[\sum_{i \in I(m)} a_{m}^{i} s_{m}\right]$
Dù $F$ est une partie finie de $E$, où les $I(m)$

sont finis, où les $a_{m}^{i} \in R$ et où tous les $a_{m}^{i} s_{m}$ sont non nuls et addibles à 1 (on suppose que toute somme partielle extraite est non nulle). Dans A, pour tout $m \in F$ et tout $i \in I(m)$, on obtient $x=\frac{b_{m}}{s_{m}}=\frac{a_{m}^{i} b_{m}}{a_{m}^{i} s_{m}}$. Le lemme montre que les $a_{m}^{i} b_{m}$ sont deux à deux addibles, et que $I^{\prime}$ on obtient $x=\frac{\sum_{\mathfrak{m}, i} a_{m}^{i} b_{m}}{\sum_{\mathfrak{m}, i} a_{m}^{i} s_{m}}=\sum_{\mathfrak{m}, i} a_{m}^{i} b_{m}$. Donc $x$ est dans $b$.

Dans la suite, on considérera un annérde $R$ de graduation donnée $\Delta$, une partie multiplicative $M$ (avec $0 \notin M$ ), l'annésde de fractions $\mathrm{R}_{M}$ et le quasi-morphisme $h: R \rightarrow R_{M}$. Pour toute partie non vide $X$ de $R$, on notera $X_{M}$ (ou $X_{p}$ si $\mathrm{H}$ est le complément d'un idéal premier $\mathfrak{p}$ ) l'ensemble : $X_{M}=\left\{\frac{x}{m} ; x \in X, m \in M\right\}$. Lorsque $X$ est un idéal, on voit (III, $\S 4 b$, lemme 6) que $X_{M}=X^{e}$. On notera $D$ et $D^{\prime}$ les zéroides, $P$ et $P^{\prime}$ les pseudozéroides, $Z$ et $Z$ ' les idéaux zérolides, $W$ et $W^{\prime}$ les idéaux pseudo-zérordes de $R$ et de $R_{M}$ respectivement.

Lemme 3 . Avec les notations précédentes, pour tout élément $\frac{\mathbf{x}}{\mathbf{s}}$ non nul de $R_{M}$, si $\frac{x}{s}$ est diviseur (resp. pseudo-diviseur) de zéro, il en est de même de $x$ dans $R$, et $x n^{\prime}$ 'est pas dans $M$.

La demière assertion est triviale. Si $\frac{x}{s} \in D^{\prime}$ est non nul (donc $x \notin \operatorname{Ker} h$ ), il existe $\frac{b}{t} \in D^{\prime}$, non nul (donc $b \notin \operatorname{Ker} h$ ), tel que $\frac{x b}{s t}=0$. Il existe $m \in M$ tel que $x(m b)=m x b=0$. Alors $b \notin$ Ker $h$ implique mb $\neq 0$, et $x$ divise zéro. 
Soit $\frac{x}{s}$ non nul un pseudo-diviseur de zéro. Il existe $n$ fractions (avec $n \geqslant 2$ ), que l'on peut supposer avoir le même dénominateur : $\frac{a}{t}, \ldots . ., \frac{a_{n}}{t}$ et vérifiant les conditions suivantes : ces fractions sont nulles et deux à deux non addibles, les $\frac{x a_{i}}{s t}$ sont tous non nuls et deux à deux addibles, et $I$ 'on $a \sum_{i=1}^{n} \frac{x a_{i}}{s t}=0$. Rendons les numérateurs addibles (III, $\S 4 a$, lemme 1) $\sum_{i=1}^{m} \frac{m a_{i}}{m s t}=0$. Il existe $u \in M$ tel que $u \sum_{i} m \times a_{i}=\sum_{i} x\left(m u a_{i}\right)=0$. Les conditions ci-dessus montrent que $m u a_{i} \neq 0$ pour tout $i$, que deux produits $m u a_{i}$ ne sont pas addibles, que les $x\left(m u a_{i}\right)$ sont tous non nuls et deux à deux addibles. Donc $x$ est un pseudo-diviseur de zéro. $\mid \begin{aligned} & \text { Lemme } 4 . \text { On a les relations : } \\ & (1) \quad D^{\prime} \subset(D \ldots M)_{M} \text { et } P^{\prime} \subset(P \ldots M)_{M} \\ & (3) \text { et, si } \omega(M) \text { associe dans } \Delta, Z^{\prime} \subset\left[(D \ldots M)_{L}\right]^{e}=\left[(Z \ldots M)_{L}\right]^{e} \\ & \text { et } W^{\prime} \subset\left[(P \ldots M)_{L}\right]^{e}=\left[(W \ldots M)_{L}\right]^{e} .\end{aligned}$

(1) résulte aussitôt du lemme 3.

Pour $x \in R$ et $s, m \in M$, les éléments $\frac{x}{s}$ et $\frac{m x}{s}$ sont ensemble nuls, ou diviseurs de zéro, ou pseudo-diviseurs de zéro. Comme $h(x)=\frac{s x}{s}$, on a en particulier $\bar{h}^{\prime}\left(D^{\prime}\right)=\left\{x \in R ; \frac{x}{s} \in D^{\prime}\right\}$, et la relation similaire pour $P^{\prime}$. Le lemme 3 montre (2).

On a d'abord $W^{\prime c}=\bar{h}^{-1}\left(W^{e}\right) \subset \bar{h}^{-1}\left(P^{\prime}\right) \subset P_{0}$. M. On sait (III, $\S 5 b$, lemme 5) que $W^{C}$ est la somme (et la réunion) de Ker $\mathrm{h}$ et d'un idéal large $E$. Comme $\mathrm{E}$ est contenu dans le noyau large $(P . . M)_{L}$, on en déduit : $W^{\prime}=W^{{ }^{C e}}=(\operatorname{Ker} h+E)^{e}=E^{e} \subset\left[(P \ldots M)_{L}\right]^{e}$. Remarquons que $(1)$ implique $W^{\prime} \subset\left[(P \ldots M)_{M}\right]_{L}$. Tout est similaire pour $Z^{\prime}$. Enfin, les égalités dans (3) sont évidentes.

Lemme 5. Soient $R$ un anné avec $0 \notin M$. I'annélde $R_{M}$ est noethérien.

Cela résulte de (III, $\hat{\oint} 4 b$, th. 3d) et de la c.c.c. dans $R_{0}$ Les lemmes 3 et 4 de (III, $\S 4 a$ ) montrent alors ceci :

Corollaire. Soient $\mathrm{R}$ un annérde unitaire, et $\mathrm{M}$ une partie multiplicative, avec $0 \notin M$. Si $R$ est noethórien fort, $R_{M} I^{\prime}$ est aussi. Si $\bar{R}$ est noethérien, $\overline{\left(\mathrm{R}_{\mathrm{M}}\right)} \mathrm{I}$ 'est aussi. 
Théorème 2. Soient $\mathrm{R}$ un annérde, et $\mathrm{M}$ une partie multiplicative $(0 \notin M)$. Soit a un idéal admettant une représentation primaire réduite $a=\bigcap_{i=1}^{n} q_{i}$. Supposons que, pour $1 \leqslant i \leqslant \underset{r}{r}$ on ait $q_{i} \cap m=\phi$, et que pour $r+1 \leqslant j \leqslant n, q_{j} \cap M \neq \varnothing$. Alors $a^{e}=\bigcap_{i=1}^{r} q_{i}^{e}$ est une représentation primaire réduite de $a^{e}$, et $a^{e c}=a_{d}$ est $I^{\prime}$ intersection des composantes primaires de $a$ qui ne rencontrent pas $M$.

I,a démonstration est identique à celle de [6], p. 225.

Théorème 3 . Soit un annérde unitaire $\mathrm{R}$ noethérien fort (resp. dont $\bar{R}$ est noethérien), et soit $M$ une partie multiplicative $(0 \notin \mathbb{M})$. Les idéaux suivants sont égaux : (1) le noyau du quasi-morphisme $R \rightarrow R_{M^{*}}$ (2) I'intersection $\mathrm{N}$ des idéaux primaires de $\mathrm{R}$ qui sont disjoints de $\mathrm{M}$. (3) I'intersection $N^{\prime}$ des composantes primaires de (0) dans $R$ qui sont disjointes de $M$.

La démonstration est identique à celle de [6], p. 226.

Corollaire. Soit un annérde unitaire $\mathrm{R}$ noethérien fort (resp. dont $\bar{R}$ est noethérien), de graduation $\Delta$, et soit $M$ une partie multiplicative de $R$ $(0 \notin M)$. Supposons que $\boldsymbol{\omega}(M)$ associe dans $\Delta_{0}$. Soient $L^{\prime}$ un idéal large de $R_{M}$, et $h$ le quasi-morphisme $R \rightarrow R_{M^{*}}$. Alors $L^{\prime C}$ est la réunion et la somme de I'enveloppe large de $\bar{h}^{-1}\left(L^{\prime} \ldots\{0\}\right)$ et de $\hat{j} q_{j}$, les $q_{j}$ étant les composantes primaires de (0) qui ne rencontrent pas $\mathrm{M}$.

On complète (III, $\S 5$, lemme 5) grâce au théorème précédent. Fractions relatives à un idéal premier. Soient $R$ un annérde et $p$

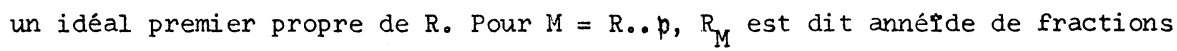
relatif à $p$ et noté $R_{p}$ On considère $l^{\prime}$ extension par $h: R \rightarrow R_{p^{*}}$. Rappelons les résultats pour ce cas particulier :

Théorème 4. Soit $p$ un idéal premier d'un annélde $R$. Si a est un idéal de $R, p$ contient $a$ si et seulement si $\mathfrak{a}^{e}$ est différent de $R_{p}$. L'application $\boldsymbol{a} \rightarrow \boldsymbol{a}^{e}$ est une bijection entre l'ensemble des idéaux premiers (resp. primaires) contenus dans $\mathfrak{p}$, et celui des idéaux premiers (resp. primaires) de $R_{p}$. La condition $\frac{a}{s} \in p^{e}$ équivaut à $a \in p$. L'idéal $p^{e}$ est l'ensemble des éléments non inversibles de $R_{p}$ et est $l$ 'unique idéal maximal. Si l'idéal a de $R$ admet une décomposition primaire réduite, $\mathfrak{a}^{\text {ec }}$ est $I^{\prime}$ intersection des composantes primaires de a qui sont contenues dans $p$. Si $R$ est unitaire et noethérien fort (rcsp. et si $\bar{R}$ est noethérien), le noyau de $h$ est I'intersection des composantes primaires de (0)(resp. des idéaux primaires de R) qui sont contenues dans p.

Théorème 5. Soient un annérde unitaire $\mathrm{R}$ noethérien fort (resp. dont $\bar{R}$ est noethérien) et $p$ un idéal premier propre de $R$. L'intersection $H$ des idéaux p-primaires est égale à $\left[\bigcap_{n=1}^{\infty}\left(p^{e}\right)^{n}\right]^{c}$. 
Les idéaux $\left(p^{e}\right)^{n}$ sont $p^{e}$-primaires (III, $\S 3$, corollaire 2 du th 5); puis, $R_{p}$ étant noethérien, tout idéal $p^{e}$-primaire contient $I^{\prime} u n$ des $\left(p^{e}\right)^{n}$ (IV, $\S 1$, th. 1). Donc, l'intersection dans $R_{p}$ des idéaux $p^{e}$-primaires est $\bigcap\left(p^{e}\right)^{n}$. La contraction commute avec une intersection quelconque. Comme il y a $n=1$

bijection entre l'ensemile des idéaux p-primaires et l'ensemble des idéaux $p^{e}$-primaires (III, $\S 3$, corollaire 2 du th. 4 ), on en déduit que l'intersection H des idéaux p-primaires est la contraction de l'intersection des idéaux $p^{e}$-primaires.

Corollaire 1. Avec les hypothèses et les notations du théorème 5 , supposons que $\boldsymbol{\omega}\left(R_{\bullet} \cdot p\right)$ associe dans $\Delta$. Alors, $H$ est contenu dans $\left(p_{L}\right)^{\text {ec }}$. En particulier, si $p$ est étroit, $H$ est l'intersection Ker $h$ des composantes primaires de $(0)$ contenues dans p.

Grace à $(v, \S 2$, corollaire $2 d u$ th. 3$)$, on majore $\bigcap_{n}\left(p^{e}\right)^{n}$ par $\left(p^{e}\right)_{L}=\left(p_{L}\right)^{e}(I I I, \S 5$, lemme 8$)$. Si p est étroit, on a donc $\cap\left(p^{e}\right)^{n}=(0)$, d'où $\mathrm{H}=$ Ker $h$ par contraction.

Appelons idéal pseudo-zérorde de $p$ le plus grand idéal large $p_{W}$ contenu dans $p \cap P$, ou, ce qui est équivalent, dans $p \cap w$. On a $p_{W}=(p \cap W)_{L}=p_{L} \cap w$. Notons $w^{\prime}$ I'idéal pseudo-zérofide de $R_{p}$; il est contenu dans $p_{L}^{e}$.

Corollaire 2. Avec les hypothèses et les notations du théorème 5 , supposons $\Delta$ associative. Alors, $H$ est contenu dans $\left(p_{W}\right)^{\text {ec }}$. En particulier, si $p \cap W$ est étroit, $\mathrm{H}$ est l'intersection Ker $\mathrm{h}$ des composantes primaires de ( 0 ) contenues dans $p$.

La remarque $(10)(v, \S 2)$ entraine $\bigcap_{n}\left(p^{e}\right)^{n} \subset w^{\prime}$, et le lemme 4 montre que $W^{\prime}=\left[(p \cap w)_{L}\right]^{e}=\left(p_{W}\right)^{e}$, d'où le résultat.

Corollaire 3 . Avec les hypothèses et les notations du théorème 5 , supposons $\Delta$ associative. Soit G I'idéal large 1-irrégulier (V, $\S 2$, remarque(2)). $L$ 'intersection des idéaux $p$-primaires contenant $G \cap p$ est égale à l'intersection des composantes primaires de $G \cap p$ contenues dans $p$. En particulier, si $G \cap p=(0), H$ est l'intersection des composantes primaires de $(0)$ contenues dans p.

Posons $\mathfrak{b}=G \cap \mathfrak{p}, S=R / \mathfrak{b}$ et $\pi=\mathfrak{p} / \mathfrak{b}$; ce dernier est évidemment un idéal premier. Les éléments de I'annérde de fractions $S_{\pi}$ sont 1-réguliers : ceux de l'idéal maximal $\pi^{\mathrm{e}}=\pi_{\pi}$ par définition de $\pi$, et ceux hors de $\pi^{\mathrm{e}}$ comme inversibles. Enfin, $1-\pi^{e}$ est sans diviseur de zéro. On a donc $\bigcap_{n=1}^{\infty}\left(\pi^{e}\right)^{n}=(0)$, et l'intersection des idéaux $\pi$-primaires de $S$ est I'intersection des idéaux primaires de $(0)$ dans $S$ contenus dans $\pi$ (théorèmes 5 et 4 ). Enfin 
(III, $\S 4$, th. 7) appliqué à I'homomorphisme surjectif $R \rightarrow R / b=S$, achève la démonstration.

$\mid \begin{aligned} & \bar{R} \\ & \text { d } \\ & \mathrm{v} \\ & \mathrm{z} \\ & \text { ex }\end{aligned}$

Théorème 6. Soit un annède unitaire $\mathrm{R}$ noethérien fort (resp. dont $\bar{R}$ est noethérien), et dont la graduation $\Delta$ est associative. Soient $p_{1}, \ldots, p_{n}$ des idéaux premiers de $\mathrm{R}$ dont aucun $\mathrm{n}^{\prime}$ est idéal premier isolé de (0). Supposons vérifiée l'une des conditions suivantes : (1) pour tout i, l'idéal pseudozérorde de chaque $\mathfrak{p}_{i}$ est (0); (2) I'idéal pseudo-zérorde $W$ de $R$ est (0); (3) chaque $p_{i}$ est étroit ; (4) I'idéal de 1-irrégularité G de $R$ est (0).

Alors, il existe un idéal dont les idéaux premiers associés sont exactement $p_{1}, \ldots, p_{n}$.

Les corollaires du théorème 5 montrent que, dans chacun des cas $(1,2,3,4)$ envisagés, et pour tout $i=1, \ldots, n, 1$ 'intersection des $i d e ́ a u x$ $\mathfrak{p}_{i}$-primaires de $R$ est $l$ 'intersection des idéaux primaires de $(0)$ contenus dans $p_{i} \cdot$

Procédons par récurrence sur $n\left(\right.$ pour $n=1$, prendre $\left.p_{1}\right)$. Pour $n \geq 2$, on peut supposer que $p_{n}$ est maximal parmi les $\mathfrak{p}_{i}$ donnés, et que n-1

$\mathbf{b}=\bigcap_{i=1} q_{i}$ est une représentation réduite, les $q_{i}$ étant $p_{i}$-primaires. si $b$ était contenu dans tout idéal $p_{n}$-primaire, il le serait dans leur intersection, donc en particulier dans une composante primaire isolée $q$ de $(0)$, donc enfin dans $p=\sqrt{q} \cdot$ Par suite $(v, \S 1$, remarque 2$)$, $p$ contiendrait 1 'un des idéaux $p_{1}, \ldots, p_{n-1}$ et lui serait égal, en contradiction avec les hypothèses. Donc il existe un idéal $\mathfrak{p}_{n}$-primaire $\mathfrak{q}_{n}$ ne contenant pas $b_{0}$

Posons $a=b \cap q_{n}=\bigcap_{i=1}^{n} q_{i}$. Si cette décomposition n'était pas réduite, $\underset{n}{\operatorname{puisque}} q_{n} \not \supset b, I$ 'un des autres idéaux primaires, $q_{1}$ par exemple, contiendrait $\bigcap_{i=2} q_{i}$. Mais $q_{1}$ ne contient pas $q_{n}$ ( $\operatorname{sinon} p_{1} \supset p_{n}$ et $p_{n}$ ne serait pas maximal dans les $p_{i}$ distincts); il en résulte que $q_{1}$ contiendrait $\bigcap_{i=2} q_{i}$, et la décomposition de $\mathbf{b}$ ne serait pas réduite, une contradiction.

Définition 1. Dans un monorde $\Delta$, un $\Delta$-idéal $H$ est dit premier si et seulement si pour tous $\alpha, \beta \notin \mathrm{H}$ on a $\alpha \beta \notin \mathrm{H}$.

Définition 2. Un idéal $\mathfrak{J}$ d'un annérde $\mathrm{R}$ est dit quasi-prèmier si et seulement si pour tous $a, b \notin \Im$, la relation $a b \in \Im$ implique $a b=0$.

Lemme 6. Soient un annélde $R$ de graduation associative $\Delta$, et ses idéaux zérolde $Z$ et pseudo-zéroide $W$. Alors, $\omega(Z)$ et $\omega(W)$ sont des $\Delta$-idéaux premiers, et les idéaux $\mathrm{Z}$ et $\mathrm{W}$ sont quasi-premiers.

Soient deux grades $\alpha, \beta \in \Delta . \omega \omega(W)$. Par définition de $W$, il existe des multiples $\lambda \alpha$ (ou $\alpha$ ) et $\mu \beta$ (ou $\beta$ ) respectivement de $\alpha$ et $\beta$, et il existe 
des éléments $a \in R_{\lambda \alpha}$ et $b \in R_{\mu \beta}$ tels que $a, b \notin P$. On voit aisément que $R_{0} P$ est une partie stable du monorde $\mathrm{R}$. Cela entraine ab $\notin \mathrm{P}$; comme $\omega(\mathrm{ab})=\omega(\mathrm{a}) \omega(\mathrm{b})=(\lambda \alpha)(\mu \beta)=(\lambda \mu)(\alpha \beta)$, on obtient $\alpha \beta \notin \omega(\mathrm{w})$. Par suite, $\omega(\mathrm{W})$ est premier. Si $c \notin \mathrm{w}, \mathrm{d} \notin \mathrm{w}$ et si $c d \neq 0$, on a $\omega(\mathrm{cd})=\omega(\mathrm{c}) \boldsymbol{\omega}(\mathrm{d})$. Puisque $\omega(\mathrm{W})$ est premier, on a $\omega(\mathrm{cd}) \notin \omega(\mathrm{W})$, donc $c d \notin \mathrm{W}$, et l'idéal $\mathrm{w}$ est presque premier. La démonstration pour $D$ et $Z$ est similaire.

Lemme 7. Soient $R$ un annérde, $L$ un idéal large quasi-premier, $p$ un idéal premier, et $q$ un idéal p-primaire. Alors $q \cup L=q+L$ est un idéal primaire, et $p \cup L=p+L$ est son idéal premier.

Utilisons (III, $\S 3$, th.5). Pour tout $b \in(p \cup L)$..L $\subset p$, il existe $m \geq 1$ tel que $b^{m} \in q \subset q \cup L$. Soient $a$ et $b$ tels que $a b \in q \cup L$ et $a \notin q \cup L$. On $a$ ou bien $a b \in q$ et par suite $b \in p$ puisque $q$ est primaire; ou bien ab $\notin q$, donc $a b \in L . .\{0\}$, et enfin $L$ presque premier implique $b \in L$.

Lemme 8. Soient un annérde unitaire $R$ noethérien fort (resp. dont $\bar{R}$ est noethérien), $W$ son idéal zéroide, et $(0)=\bigcap_{i=1}^{n} q_{i}$ une décomposition primaire
réduite de $(0)$, d'idéaux premiers associés $p_{i}(i=1, \ldots, n)$. Alors
$n$
$\begin{gathered}n \\ n \\ i=1\end{gathered}\left(q_{i} \cup w\right)$ est une décomposition primaire de $w$, dont les idéaux premiers
sont les $p_{i} \cup w$.

On a le mème résultat en remplaçant $\mathrm{W}$ par $\mathrm{Z}$.

En erfet, chaque $q_{i} \cup W$ est un idéal primaire pour $p_{i} \cup w$, et on a : $n_{i}\left(q_{i} \cup w\right)=\left(\underset{i}{n} q_{i}\right) \cup w=(0) \cup w=w$.

Théorème 7. Soit un annérde unitaire $R$ noethérien fort (resp. dont $\bar{R}$ est noethérien) et dont la graduation $\Delta$ est associative. Soient $p_{1}, \ldots, p_{n}$ des idéaux premiers distincts, contenant l'idéal pseudo-zéroide $W$, et dont aucun n'est idéal premier isolé de $\mathrm{w}$. Alors il existe un idéal dont les idéaux premiers associés sont exactement $p_{1}, \ldots, p_{n}$.

Que $p_{i}$ ne soit pas idéal premier isolé de $w$, équivaut à ce que, pour tout idéal premier isolé $\pi$ de $(0), p_{i}$ ne soit pas contenu dans $\vdots U \pi_{0}$

Pour tout idéal premier $p_{i}$, l'intersection de ses idéaux $p_{i}$-primaires est (corollaire $2 d u$ th.5) contenu dans $\left(p_{i W}\right)^{\text {ec }}=W^{e C}$ (extension par $R-R_{p_{i}}$ ), qui est égal (th. 4) à l'intersection des composantes primaires de $W$ qui sont contenues dans $p_{i}$. Ceci étant, la démonstration est identique à celle du théorème 6 , en remplaçant (0) par $W$.

Remarquons que si a est un idéal quasi-premier d'un annérde $R$, et si $\alpha \in R / a$ est un diviseur de zéro, tout élément a de la classe $\alpha$ est un diviseur de zéro dans 2. En effet, $a \notin a$ et il existe $b \notin a$ tel que $(a+a)(b+a) \subset a$, donc que $a b \in a$. On a donc $a b=0$, puisque a est quasi-premier. 
[1] N. BOURBAKI - Algèbre. Chap. II (3e éd.) Hermann - Paris 1962.

[2] N. BOURBAKI - Algebre Commutative - Chap. III et IV - Hermann - Paris 1951.

[3] M. KRASNER - Théorie des Corps valués (vol. 1, Exposés 1-4).

[4] M. KRASNER - Théorie des Corps valués (vol. 2, Exposé 5). Séminaire, année 1953-54. Secrétariat mathématique, 11 rue Pierre Curie - Paris 1956.

[5] E.S. LJAPIN - Semi groups - American Mathematical Society Providence - Rhode Island (1953).

[6] 0. ZARISKI - P. SAMUEL - Commutative Algebra (vol. I). Van Nostrand Company, Princeton, New-Jersey (1958).

[7] SAGASTUME BERRA - Systemas de homogeneldad - Univ. Nac. La Plata. Publ. Fac. Ci. Fisico Mat., Série Segund a - Rev. 6, n॰ 5, 1959, pp. 5-17. 
Pages

E..F $=\{x \in E ; x \notin F\} . . . . . .7$

$\mathrm{E}^{*}=\mathrm{E} . .\{0\} . . \cdot . \cdot . \cdot$. . 7

$\overline{\mathrm{M}}^{\mathrm{S}}, \overline{\mathrm{A}}^{\mathrm{S}}, \Lambda^{\mathrm{S}} \cdot \ldots \cdot \cdot . \cdot . \cdot 55,56$

$\mathrm{A}(\mathrm{a}), \mathrm{M}(\mathrm{x}) \cdot \ldots 20,21,34,35$

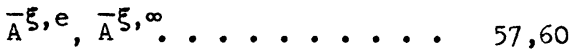

$X+Y, F+G$....... 23,42

$\overline{\mathrm{A}}(\mathrm{s}, \mathrm{D}), \overline{\mathrm{A}}(\mathrm{S}, \mathrm{D}) . . . . .60,61$

$\mathrm{A}_{\mathrm{d}}, \mathrm{M}_{\mathrm{C}^{\cdot}} \cdot \cdot \cdot \cdot \cdot \cdot \cdot \cdot \cdot \cdot 26$

$[m, L],[m] . . . . . .67$

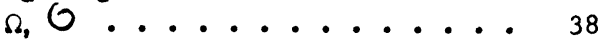

$a_{\mathrm{d}^{\circ}} \cdot \cdot \cdot \cdot \cdot \cdot \cdot \cdot \cdot \cdot \cdot 88$

$\omega(\mathrm{a}), \Delta . . . . . . .25,26,40$

$\mathrm{El}(\mathrm{P}), \mathrm{Fl}(\mathrm{P}) \cdot$. . . . . . 94

$\overline{\mathrm{A}}, \overline{\mathrm{M}} \cdot$. . . . . . 23,39

$\mathrm{P}_{\mathrm{L}}, \mathrm{P}_{\mathrm{F}^{\circ}} \cdot$ ・ ・ ・ • • • • 94

$\overline{\mathrm{M}}^{\mathrm{f}}, \overline{\mathrm{M}}^{\mathrm{a}}, \overline{\mathrm{A}}^{\mathrm{f}}, \overline{\mathrm{A}}^{\mathrm{x}} \cdot \ldots . . .47,48,49$

D, Z, P, W. . . . . . 124, 125

$\Lambda^{f}, \Lambda^{\mathrm{x}} \cdot \ldots \cdot \ldots$

G. ......... 127

$P_{W} \cdot \cdot \cdot \cdot \cdot \cdot \cdot \cdot \cdot \cdot \cdot \cdot \cdot$

INDEX TERMINOLOGIQUE

Absorption. . . . . . 114

Agglutinateur . . . . . . 128

Agglutinateur(a)-associé . . . 128

Agglutiné . . . . . . 35,47

Agglutiné large . . . . . 36,51

Agglutiné simple . . . . . 52

Anel . . . . . . . . 37

Annélde ......... 20

Artinien . . . . . 24, 102

$\xi$ associe dans $\Delta . . . . . .59$

Associés (éléments - . . . 109

Charpente......... 110

Co-facteurs . . . . . . 35

Comaximaux . ....... 76

Combinaison linéaire. • . • 41

Corporde.......... 21

Couche. ....... . . . 114

Domaine. . . . . . 36,52

Enveloppe large. •. . . . 94

Factorisation (suite de - . . 112

Fermeture divisible... . . 88

Fort....... 34,46, 105

Grade............ 26
Grade propre........ 25

Grade nul, vide • • • • • 26

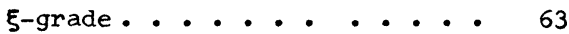

Graduation . . . . . 26,40

Graduation forte, très forte . 105

Gradué (anneau -) . . . . 23

Graphe . •. •. •. •. 114

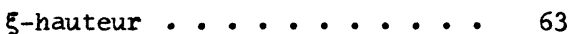

Homomorphisme . . . . . 32,46

Idéal . . . . . . 24,40

Idéal étroit . . . . . . 94

Idéal faiblement large. • . . 92

Idéal large • • • • • • 92,93

Idéal $\Delta$-large . . . . . 93

Idéal premier. • • • • • 77

Idéal primaire. • • • • • 78

Idéal pseudo-zéroide. . • 125,138

Idéal zéroide . . . . . 125

Intègre (annéide - • • • 36

Intégroide . . . . . . . 21

1 -irrégulier . • . . . • 127

Isomorphisme . • . . . 46,69,72

Libre (monorde - . - • . 115 
Local (annéide - . . . 130

Large . . . . . . . 92,93

Largement premier . . . . . 78

Largement primaire...... 78

Milieu........ 38,39

Modulaire .......... 42

Modulolde .......... 21

Monel ......... 38

Monolde libre ........ 115

Monomorphisme ...... 69,73

Nilpotent par rapport à $\Omega$. . 108

Noethérien . . . . 24,97, 109

Noyau large ........ 94

Pente.... ...... 62

§-polynôme.......... 62

Premier à........... 88

Premier . • . •...... 77

Premier (largement -) . . . 78

Premier (strictement - . . - 78

Primaire........ 78

Primaire (1 argement -) . . 78

Primaire (strictement _-) . . 78
Prolongement d'une graduation. . 61

Pseudo-diviseur. . . . ( 35,52

Pseudo-inversible. . . . 35,52

Pseudo-zérolde. . . . . 125,138

Quasi-directe (somme - . . . 39

Quasi-domaine. . . . . 35,52

Quasi-gradué . . ...... 40

Quasi-morphisme..... 32,46

Quasi-premier... . . . . 139

Quasi-régulier . . . 34,46, 105

Régulier ....... 34,46

1-régulier . . . . . . . 127

Représentation primaire . . . 122

Résidus . . . . . . . 112

Stationnaire . . . . . 105

Strict ........ . 34,46

Strictement premier...... 78

Strictement primaire ..... 78

Suite de factorisation . . . 112

Transverse ......... 115

Zérotde ......... 125

(Texte reçu le 24 juin 1970)

Marcel CHADEYRAS

Faculté des Sciences

34, Avenue Carnot

63 - CLERMONT-FERRAND 